\title{
Kinetische Plasmaprozesse und Welle-Teilchen-Wechselwirkung von Ionen im schnellen Sonnenwind
}

Theoretische Untersuchung und Auswertung von Helios Beobachtungen

\author{
Dissertation \\ zur Erlangung des Doktorgrades \\ der Mathematisch-Naturwissenschaftlichen Fakultäten \\ der Georg-August-Universität zu Göttingen
}

vorgelegt von

Michael Heuer

aus Peine

Göttingen 2005 
D7

Referent: Prof. Dr. F. Kneer

Korreferent: Prof. Dr. E. Marsch

Tag der mündlichen Prüfung: 23. September 2005 


\section{Inhaltsverzeichnis}

Zusammenfassung $\quad 5$

1 Einleitung $\quad 7$

1.1 Phänomenologie und Modelle des Sonnenwindes . . . . . . . . . . . 7

1.2 Quasi-lineare Diffusion und Mikroinstabilitäten im Sonnenwind . . . . . 12

1.3 Zielsetzungen und Aufbau der Arbeit . . . . . . . . . . . . . . . . . . 14

2 Grundlagen 17

2.1 Theorie mittlerer Felder . . . . . . . . . . . . . . . . . . 17

2.2 Kinetische Plasmawellen und ihre Instabilitäten . . . . . . . . . . . 18

2.2.1 Dispersion bei paralleler Ausbreitungsrichtung . . . . . . . . . 19

2.2.2 Dispersion für Bi-Maxwell-Verteilungen . . . . . . . . . . 21

2.2.3 Mikroinstabilitäten ... . . . . . . . . . . . 23

2.3 Quasi-lineare Diffusion . . . . . . . . . . . . . . . 28

3 Meßdaten $\quad 33$

3.1 Helios . . . . . . . . . . . . . . . . . . . 33

3.1 .1 Das Plasmaexperiment . . . . . . . . . . . . 34

3.1.2 Abtrennung der $\alpha$-Teilchen . . . . . . . . . . . . . 38

3.1.3 Das Sondenpotential und die Elektronen-Messung . . . . . . . . 40

3.2 Datenauswahl . . . . . . . . . . . . . . . . . . . . . 44

4 Modellierung der Geschwindigkeitsverteilungen der Protonen 49

4.1 Entwicklung nach Kernfunktionen . . . . . . . . . . . . . . . . 50

4.2 Optimale Glättungsparameter . . . . . . . . . . . . . . . . . . . . 51

4.3 Anwendung auf die Meßdaten . . . . . . . . . . . . . . . . 53

5 Numerische Methode zur Lösung der kinetischen Dispersionsbeziehung 59 
5.1 Bestimmung der Lösungen der Dispersionsbeziehung bei fixiertem Wellenvektor ....................... 60

5.2 Bestimmung der Abhängigkeit der Lösungen der Dispersionbeziehung vom Wellenvektor . . . . . . . . . . . . . . . 61

5.3 Bestimmung der Parameterabhängigkeit von Moden maximaler Anwachsrate ........................... 62

6 Untersuchung von Mikroinstabilitäten im schnellen Sonnenwind

6.1 Zerlegung der Geschwindigkeitsverteilungen der Protonen in Kern und Strahl ......................... 66

6.2 Vereinfachung der reduzierten Verteilungen der Protonen . . . . . . . . . 69

6.3 Instabilitäten bei schräger Ausbreitungsrichtung . . . . . . . . . . . . . 70

6.3.1 Strahl-Instabilitäten . . . . . . . . . . . . . . . 72

6.3.2 Spiegel-Instabilität . . . . . . . . . . . . . . 8 80

6.4 Instabilitäten bei paralleler Ausbreitungsrichtung . . . . . . . . . . . 82

7 Untersuchung von Diffusionsplateaus im schnellen Sonnenwind 91

7.1 Diffusionsplateaus im Kern der Verteilung der Protonen . . . . . . . . . . 92

7.2 Diffusionsplateaus im Strahl der Verteilung der Protonen . . . . . . . . . 99

$\begin{array}{llr}8 & \text { Schlußfolgerungen und Ausblick } & 103\end{array}$

A Radiale Profile der Plasmaparameter zu den untersuchten Strömungen schnellen Sonnenwindes

B Asymptotischer MISE für die nach Gaußschen Kernfunktionen entwickelten Geschwindigkeitsverteilungen

C Symmetrisierung und Reduktion der nach Gaußschen Kernfunktionen entwickelten Geschwindigkeitsverteilungen

D Dispersionsbeziehung für die nach Gaußschen Kernfunktionen entwickelten reduzierten Geschwindigkeitsverteilungen

Literaturverzeichnis 


\section{Zusammenfassung}

Wesentliche Eigenschaften des schnellen Sonnenwindes lassen sich nur durch eine Wechselwirkung der Teilchen des Windes mit Plasmawellen erklären. Die quasi-lineare Näherung dieser Wechselwirkung, welche in Modellen des Sonnenwindes herangezogen wird, geht von der statistischen Beschreibung des Zustands des Plasmas aus. Diese Näherung beinhaltet die Annahme einer Trennung der Skalen der Fluktuationen und der mittleren Zustandsgrößen. Die nicht-lineare Wechselwirkung zwischen den Plasmawellen wird ausgehend von der Vorstellung einer schwachen Turbulenz vernachlässigt. In dieser Arbeit habe ich mich der Frage gewidmet, inwieweit diese einfache Näherung sich auf den schnellen Sonnenwind anwenden läßt und ob die ihr zugrunde liegenden Annahmen über den Zustand des schnellen Sonnenwindes gerechtfertigt sind. Dafür habe ich die auf der Raumsonde Helios 2 im solaren Minimum des Jahres 1976 gewonnenen Plasmamessungen verwendet. Die Auswertung erstreckt sich auf den gesamten Bereich zwischen dem Perihel und dem Aphel von Helios 2 bei 0.3 AE bzw. 1 AE. Es wurden zwei Ansatzpunkte verfolgt. Der im schnellen Sonnenwind beobachtete Protonenstrahl sowie die ebenfalls dort beobachtete Temperaturanisotropie des Kerns der Geschwindigkeitsverteilungen der Protonen können zur linearen Instabilität von Plasmawellen führen. Im Rahmen der quasi-linearen Theorie müssen die linearen Anwachsraten der instabilen Moden in dem stationären Zustand des schnellen Windes von der Größenordnung des Kehrwertes der typischen Expansionszeitskala sein, welche eine Grenze marginaler Instabilität vorgibt. Es wurde das Auftreten von linearen Instabilitäten im schnellen Sonnenwind durch eine direkte Berechnung ihre Anwachsraten aus den gemessenen Geschwindigkeitsverteilungen der Protonen, $\alpha$-Teilchen und Elektronen untersucht. Der zweite Ansatzpunkt dieser Arbeit bestand in der Untersuchung der Geschwindigkeitsverteilungen der Protonen des schnellen Sonnenwindes auf Diffusionsplateaus, wie sie von der quasi-linearen Näherung vorhergesagt werden. Die Ausbildung von Diffusionsplateaus im Kern der Verteilungen vermag seine Temperaturanisotropie zu erklären. Die Auswertung der Plasmamessungen des schnellen Sonnenwindes auf Helios 2 führte zu den folgenden Ergebnissen:

- Die Temperaturanisotropie des Kerns der Geschwindigkeitsverteilungen des Protonen verursacht für nahezu alle gemessenen Verteilungen eine lineare Instabilität der auswärts laufenden Alfvén-Moden im Bereich der Zyklotronfrequenzen der Ionen. Die Anwachsraten der instabilen Moden übersteigen die Grenze der marginalen Instabilität um zwei Größenordnungen.

- Der Protonenstrahl führt nur für ungefähr $10 \%$ aller gemessenen Geschwindigkeitsverteilungen zu einer linearen Instabilität. Die beobachteten Anwachsraten der 
Strahl-Instabilitäten dürfen als marginal bezeichnet werden.

- Die in Bezug auf die Sonne einwärts gerichtete Hälfte der Geschwindigkeitsverteilungen der Protonen zeigt eine gute und mit der Entfernung zur Sonne unveränderliche Übereinstimmung mit den Diffusionsplateaus, welche durch die quasi-lineare Wechselwirkung mit den auswärts laufenden Alfvén-Moden im Bereich der Zyklotronfrequenzen der Ionen vorhergesagt werden.

- Die Abweichung der Geschwindigkeitsverteilungen von den Diffusionsplateaus in der auswärts gerichtete Hälfte des Kerns, welche durch die quasi-lineare Wechselwirkung mit bestimmten einwärts laufenden Zyklotronmoden enstehen sollten, nimmt mit der Entfernung zur deutlich Sonne zu. Sie ist bereits im Perihel von Helios größer als in der einwärts gerichteten Hälfte des Kerns.

- Innerhalb des Protonenstrahls läßt sich keine Übereinstimmungen mit möglichen quasi-linearen Diffusionsplateaus beobachten. 


\section{Einleitung}

\subsection{Phänomenologie und Modelle des Sonnenwindes}

Der erste Hinweis auf die Existenz kontinuierlich und in alle Richtungen von der Sonne abströmender Teilchen fand sich in den Beobachtungen von Kometenschweifen. Biermann (1951) postulierte den Sonnenwind bzw. eine Korpuskularstrahlung, wie er den Sonnenwind nannte, um Beschleunigungen von Schweifelementen zu erklären, die der Strahlungsdruck allein nicht erzeugen kann. Parker (1958) zeigte daraufhin, dass das Bild einer hydrostatischen Korona unter der Annahme realistischer Wärmeleitung zu einem physikalischen Widerspruch führt. Der Gasdruck der Korona bliebe im Unendlichen wesentlich größer als der verschwindend geringe Gasdruck des interstellaren Mediums. Er betrachtete das einfache Modell einer stationären sowie isothermen und sphärisch symmetrischen expandierenden Korona und fand, daß es zu jeder an der koronalen Basis vorgegebenen Temperatur und Dichte nur eine einzige Lösung geben kann. Diese kritische Lösung besitzt eine Geschwindigkeit, die an der Basis kleiner als die Schallgeschwindigkeit ist und diese in einer kritischen Entfernung erreicht und übersteigt. (s. dazu Parker 1960, 1963a,b,c, 1965). Die Existenz des von Biermann und Parker vorhergesagten Sonnenwindes konnte durch Messungen auf Mariner 2 (Neugebauer und Snyder 1962) direkt nachgewiesen werden. In der folgenden Zeit waren Plasmaexperimente Bestandteil der meisten Missionen, die den interplanetaren Raum mit unterschiedlichen wissenschaftlichen Zielsetzungen durchquerten. Der Sonnenwind konnte in der inneren Heliosphäre z.B. durch Helios 1 und Helios 2 erforscht werden (Porsche 1977, Rosenbauer et al. 1977). Beobachtungen des Sonnenwindes in der äußeren Heliosphäre ermöglichten z.B. die Sonden Pioneer 10 und Pioneer 11 sowie Voyager 1 und Voyager 2 (Belcher et al. 1981). Zusätzlich gab es etliche Unternehmungen, durch die der Sonnenwind in der Erdnähe gemessen wurde. Ulysses erlaubte die Beobachtung des Sonnenwindes auch deutlich außerhalb der Ekliptik (s. Wenzel et al. 1989, Bame et al. 1992).

Der Sonnenwind besteht zum Hauptteil aus ionisiertem Wasserstoff, d.h. aus Protonen. Vollständig ionisiertes Helium ( $\alpha$-Teilchen) besitzt eine relative Häufigkeit von $n_{\alpha} / n_{p}=$ 0.01 - 0.05 (s. z.B. Marsch et al. 1982b, Aellig et al. 2001). Die Ionisationszustände anderer Ionenarten tauchen mit wesentlich geringeren Häufigkeiten auf (s. z.B. von Steiger et al. 1997). Es hat sich gezeigt, daß der Sonnenwind in zwei fundamentalen Zuständen auftritt, dem schnellen und dem langsamen Sonnenwind. Der schnelle Sonnenwind zeichnet sich durch hohe Strömungsgeschwindigkeiten $(550$ - $800 \mathrm{~km} / \mathrm{s})$, hohe Temperaturen 
$\left(1-4 \times 10^{5} \mathrm{~K}\right)$ und niedrige Teilchendichten $\left(1-5 \mathrm{~cm}^{-3}\right)$ aus ${ }^{1}$. Der langsame Sonnenwind besitzt im Unterschied dazu niedrige Strömungsgeschwindigkeiten $(250-500 \mathrm{~km} / \mathrm{s})$, niedrige Temperaturen $\left(0.25-2 \times 10^{5} \mathrm{~K}\right)$ und hohe Teilchendichten $\left(5-25 \mathrm{~cm}^{-3}\right)$. Der schnelle Sonnenwind hat seinen Ursprung in den koronalen Löchern. Er zeigt sehr einheitliche und über lange Zeiträume beständige Eigenschaften, während der langsame Sonnenwind weniger einheitlich und zeitlich sehr variabel ist (s. z.B. Axford und McKenzie 1992, 1997). Das Parker-Modell ist in der Lage, unter realistischen koronalen Randbedingungen die Geschwindigkeiten und Dichten des langsamen Sonnenwindes in der Erdentfernung vorherzusagen. Die hohen Geschwindigkeiten des schnellen Sonnenwindes können in diesem Modell nur durch hohe Temperaturen an der koronalen Basis erreicht werden (bis zu $4 \times 10^{6} \mathrm{~K}$ ). Jedoch wächst damit auch der Teilchenstrom des Windes an, so daß die vorhergesagte Dichte in der Erdentfernung im Widerspruch zu den beobachteten, niedrigen Dichten des schnellen Sonnenwindes steht. Das Modell unterscheidet nicht zwischen den einzelnen Ionenarten und den Elektronen im Wind. Es beinhaltet die Annahme eines lokalen thermodynamischen Gleichgewichts unter den Teilchenspezies, welche dieselbe mittlere Geschwindigkeit und isotrope Temperatur besitzen sollen. Ein solches Gleichgewicht kann nur durch Kollisionen der Teilchen und dem damit verbundenen Austausch von Energie und Impuls aufrechterhalten werden. Die Kollisionsfrequenz zwischen den Teilchen des Sonnenwindes sind um Größenordnungen kleiner als die Expansionsrate des Sonnenwindes, $v d \ln n / d r \approx 2 v / r \approx 10^{-5} \mathrm{~s}^{-1}$, weshalb ein lokales thermodynamisches Gleichgewicht in dieser Entfernung, aber ebenso in größerer Nähe zur Sonne, nicht angenommen werden darf. Auch die Beobachtungen haben gezeigt, daß ein solches Gleichgewicht nicht besteht. Die Elektronen besitzen im schnellen Sonnenwind eine niedrigere Temperatur als die Protonen, $T_{p} \approx 2.5 T_{e}$, und im langsamen eine höhere, $T_{e} \approx 2.5 T_{p}$ (Pilipp et al. 1981, 1987a,b). Die Temperatur der $\alpha$-Teilchen ist mit $T_{\alpha} \approx 3-6 T_{p}$ stets größer als diejenige der Protonen (Marsch et al. 1982b). Die mittlere Geschwindigkeit der $\alpha$-Teilchen übersteigt die der Protonen im schnellen Sonnenwind bis zur Größenordnung der Alfvén-Geschwindigkeit, im langsamen Sonnenwind stimmen beide im wesentlichen überein (Marsch et al. 1982b). Desweiteren sind die Geschwindigkeitsverteilungen aller Spezies grundsätzlich anisotrop. Die Temperatur einer Teilchenart parallel zum magnetischen Feld stimmt nicht mit ihrer Temperatur senkrecht dazu überein. So lassen sich Temperaturanisotropien der Protonen beobachten, die mit dem Abstand von der Sonne abnehmen und im langsamen Sonnenwind mit $T_{\perp} / T_{\|} \approx 0.3-1$ kleiner sind als im schnellen mit $T_{\perp} / T_{\|} \approx 0.5-1.5$ (Marsch et al. 1981, 1982c). Auch die $\alpha$-Teilchen weisen Temperaturanisotropien auf, $T_{\perp} / T_{\|} \lesssim 1$ (Marsch et al. 1982b). Desweiteren tritt in den Geschwindigkeitsverteilungen der Protonen häufig ein Protonenstrahl auf. Damit ist eine zweite Population von Protonen mit geringerer Teilchendichte gemeint, die sich relativ zu der Hauptpopulation auswärts und entlang des mittleren magnetischen Feldes bewegt (Feldman et al. 1973, Asbridge et al. 1974, Feldman et al. 1974, Marsch et al. 1981, 1982c). Im schnellen Sonnenwind ist der Protonenstrahl eine reguläre Erscheinung. Seine Relativgeschwindigkeiten ist im Mittel etwas größer als die Alfvén-Geschwindigkeit. Der Anteil des Strahls an der Teilchendichte der Protonen liegt zwischen 10\% und 20\% und ist im langsamen Sonnenwind größer als im schnellen. Die Hauptpopulation, d.h. der Kern der Verteilung der Protonen besitzt eine größere Temperaturanisotropie als die Gesamt-

\footnotetext{
${ }^{1}$ Die angegebenen Werte beziehen sich auf die Protonen des Sonnenwindes in der Erdentfernung.
} 
verteilung. Es lassen sich im schnellen Sonnenwind Temperaturanisotropien des Kerns von bis zu $T_{\perp} / T_{\|} \lesssim 4$ beobachten (s.a. Bame et al. 1975). Feldman et al. (1974) schlugen als Ursprung des Protonenstrahls räumlich und zeitlich variierende Bedingungen in der Quellregion des Sonnenwindes vor. Demnach könnten räumlich begrenzte Filamente schnelleren Plasmas mit der Entfernung in einen sie umgebenden langsameren Wind diffundieren. Außerdem könnte ein zu einem späteren Zeitpunkt entstehender Sonnenwindstrom einen langsameren überholen und durchdringen. Es wurde ebenfalls versucht, daß Entstehen des Protonenstrahls durch einen Prozeß im interplanetaren Raum zu erklären Livi und Marsch (1987). Die Frage, ob der Protonenstrahl solaren Ursprungs ist oder sich im interplanetaren Raum bildet, ist ungeklärt.

Aufgrund der im Sonnenwind sehr niedrigen Kollisionsraten der Teilchen modellierten Sturrock und Hartle (1966) und Hartle und Sturrock (1968) die Elektronen und Protonen im Sonnenwind als zwei unabhängige und durch Kollisionen schwach gekoppelte Flüssigkeiten mit identischen Geschwindigkeiten und Dichten. Sie nahmen isotrope aber verschiedene Temperaturen und die Gültigkeit des klassischen Wärmetransportes für die Teilchenarten an. Ihr Modell schaffte es nicht, den schnellen Sonnenwind zu erzeugen, sondern verursachte eine weitere Erklärungsnot. Für realistische Temperaturen und Dichten an der koronalen Basis sagte es einen Sonnenwind mit Geschwindigkeiten und Teilchendichten von der Größenordnung von $300 \mathrm{~km} \mathrm{~s}^{-1}$ bzw. $10 \mathrm{~cm}^{-3}$ in der Erdentfernung voraus. Die Kollisionen erwiesen sich als nicht im Stande, dem Auseinanderstreben der Temperaturen der Elektronen und Protonen aufgrund der deutlich geringeren klassischen Wärmeleitfähigkeit der Protonen entgegenzuwirken. Das Modell sagte zu große Elektronentemperaturen und sowohl für den schnellen als auch den langsamen Sonnenwind um eine Größenordnung zu niedrige Protonentemperaturen in der Erdentfernung voraus. Leer und Axford (1972) schlußfolgerten aus den Arbeiten von Sturrock und Hartle (1966) und Hartle und Sturrock (1968), daß die Protonen im Sonnenwind zusätzlich zu den Stößen mit den Elektronen geheizt werden müßten. Sie beschrieben die Protonen und Elektronen ebenso durch zwei über Kollisionen gekoppelte Flüssigkeiten mit gemeinsamer Geschwindigkeit und Dichte. Zudem nahmen sie die Gültigkeit der klassischen Wärmeleitfähigkeiten an, berücksichtigten aber die Temperaturanisotropie der Protonen und führten ad hoc eine nahe dem koronalen Rand konzentrierte Heizung der Protonen ein. Ihr Modell ergibt unter realistischen Randbedingungen Geschwindigkeiten und Teilchendichten in der Erdentfernung, die im Bereich der im langsamen Sonnenwind beobachteten Werte liegen, was Dank der Heizungsraten auch für die Protonentemperatur gilt. Die vorhergesagte Elektronentemperatur ist allerdings sowohl für den langsamen als auch den schnellen Wind zu groß, und die Temperaturanisotropien sind zu klein. Das Modell konnte den schnellen Sonnenwind nicht reproduzieren und blieb eine Erklärung der physikalischen Ursache der zusätzlichen Heizung schuldig. Durch Beobachtungen auf Helios konnte später gezeigt werden, daß sich die erforderliche Heizung der Protonen mindestens bis zur Erdentfernung fortsetzen müßte, um den nicht-adiabatischen radialen Verlauf der Temperatur und das Auftreten der Temperaturanisotropie erklären zu können (Marsch et al. 1983, Schwartz und Marsch 1983).

In einer Reihe von Arbeiten (so z.B. bereits Alfvén 1947) wurde die Dissipation von magnetohydrodynamische Wellen als Ursache der Heizung der Korona vorgeschlagen. Belcher und Davis (1971) konnten solche Wellen mit Plasmamessungen und Messungen des 
interplanetaren Magnetfeldes auf Mariner 5 im Sonnenwind nachweisen. Sie zeigten, daß es sich dabei im wesentlichen um auswärts laufende Alfvénische Fluktuationen handelt, deren mittlere Amplitude von der Größenordnung des mittleren magnetischen Feldes ist. Damit bot sich die Dissipation von Alfvén-Wellen als Erklärung für die offenbar erforderliche Heizung der Protonen an. Außerdem können Alfvén-Wellen durch ihren Wellendruck, das ist ihr mittlerer magnetischer Druck, den Sonnenwind zusätzlich beschleunigen und somit den schnellen Sonnenwind erzeugen. Belcher (1971) bezog Alfvén-Wellen in das Modell des Sonnenwindes von Parker (1960) ein. Sein Modell enthielt keine Dissipation der Wellen. Durch den berücksichtigten Wellendruck war es aber fähig, die hohen Geschwindigkeiten des schnellen Sonnenwindes zu erzeugen, ohne zugleich auch unrealistisch hohe Teilchenströme und somit Dichten in der Erdentfernung mit sich zu bringen. Hollweg (1973) erweiterte das Zweiflüssigkeiten-Modell von Sturrock und Hartle (1966) und Hartle und Sturrock (1968) um auswärts laufende Alfvén-Wellen. Er erzwang ihre Dissipation durch ein Sättigungsprinzip, wonach die Amplitude der Wellen relativ zum mittleren magnetischen Feld in einem bestimmten Entfernungsbereich zur Sonne konstant bleibt. Der damit verbundene Verlust des Energiestroms der Alfvén-Wellen diente der Heizung der Protonen. Unter realistischen Randbedingungen reproduzierte das Modell die Temperaturen der Protonen des schnellen Sonnenwindes in der Erdentfernung. Die vorhergesagte Geschwindigkeit war aber deutlich zu niedrig, und die Elektronentemperaturen überragten diejenigen der Protonen. Deshalb modifizierte Hollweg (1978) sein Modell durch eine nicht-klassische Wärmeleitfähigkeit der Elektronen (Hollweg 1974, 1976), die zu effektiv niedrigeren Wärmeströmen führte und die Elektronentemperatur schneller abfallen ließ. Dadurch konnten realistische Werte der Temperatur in der Erdentfernung erzielt werden, und wegen des größeren Gradienten des thermischen Drucks ergab sich ein schnellerer Wind (s. Jacques 1977, 1978, zu vergleichbaren Modellen).

Die Arbeiten von Belcher (1971), Hollweg (1973), Jacques (1977, 1978) und Hollweg (1978) verdeutlichten, daß von der Sonne stammende und auswärts laufende AlfvénWellen den Sonnenwind auf die beobachteten Geschwindigkeiten beschleunigen können. Dabei erlauben sie es zugleich, die hohen Geschwindigkeiten des Windes mit niedrigen Teilchendichten in der Erdentfernung und ausreichend großen Dichten an der koronalen Basis, d.h. realistischen Teilchenströmen, in Einklang zu bringen. Durch das Erzwingen einer Dissipation der Alfvén-Wellen kann auch die Temperatur des Sonnenwindes bzw. die Protonentemperatur in der Erdentfernung auf die hohen Werte im schnellen Wind gehoben werden. Eine selbstkonsistente Beschreibung des Dissipationsvorganges blieben die betreffenden Arbeiten schuldig. Eine Reduktion des Wärmetransportes der Elektronen scheint zum einen für die richtige Berechnung ihres Temperaturverlaufes und zum anderen im Zusammenspiel mit den Alfvén-Wellen für eine ausreichende Beschleunigung des schnellen Sonnenwindes nötig zu sein. Leer und Holzer (1980) untersuchten im Rahmen eines Einflüssigkeiten-Modells und unter Einbeziehung von Hollwegs Reduktion der Wärmeleitung den Einfluß der Entfernung, in der Impuls oder Wärmeenergie auf den Wind übertragen werden. Sie fanden, daß eine zusätzliche Heizung oder Beschleunigung des Windes innerhalb der kritischen Entfernung die Geschwindigkeit und die Temperatur des Sonnenwindes in der Erdentfernung kaum anhebt bzw. sogar senkt, den Teilchenstrom allerdings vergrößert. Dem entgegen erhöhen eine Heizung und Beschleunigung des Windes außerhalb der kritischen Entfernung seine Geschwindigkeit, eine Heizung 
aber auch seine Temperatur substantiell, ohne auf den Teilchenstrom einen Einfluß zu besitzen. Die von Alfvén-Wellen verursachte Heizung des Sonnenwindes tritt nach dem Sättigungsmechanismus erst außerhalb des kritischen Punktes ein, und ihr Wellendruck ist im Vergleich zum thermischen Druck und dem Gravitationspotential innerhalb der kritischen Entfernung gering. Die Beschleunigung und die Heizung des Sonnenwindes durch Alfvén-Wellen finden somit im wesentlichen außerhalb des kritischen Punktes statt, was nach Leer und Holzer (1980) erst die spezifischen Eigenschaften des schnellen Windes ermöglicht. Es wurde versucht, die bevorzugte Beschleunigung und die Heizung der $\alpha$ Teilchen ebenfalls durch Alfvén-Wellen zu erklären Marsch et al. (1982a), Isenberg und Hollweg (1983), Isenberg (1984). In diesem Zusammenhang wurde von Marsch et al. (1982a) die sogenannte quasi-lineare Näherung der Welle-Teilchen-Wechselwirkung in die Modellierung des Sonnenwindes eingeführt. Im Gegensatz zu den theoretischen Konzepten, die den bisher diskutierten Arbeiten zugrunde liegen, handelt es sich dabei um eine kinetische Beschreibung dieser Wechselwirkung in einem kollisionsfreien Plasma (s. z.B. Vedenov et al. 1962, Drummond und Pines 1962, Kennel und Engelmann 1966, Sagdeev und Galeev 1969, Davidson 1972, Galeev und Sagdeev 1983a). Sie ermöglicht es, sowohl die Beschleunigung der Teilchen als auch ihre Heizung zu berechnen, und es entfällt die Notwendigkeit eines Sättigungsprinzip, um die Dissipation der Wellen auszudrücken. Die Wellenmoden müssen eine Resonanzbedingung erfüllen, um die Teilchen heizen zu können. Die resonanten Wellen besitzen Frequenzen im Bereich der Zyklotronfrequenzen der Ionen. Sie stellen eine Fortsetzung der von Belcher und Davis (1971) beobachteten Alfvén-Wellen in diesen Frequenzbereich hinein dar. Mit wachsender Entfernung zur Sonne nehmen der Betrag des interplanetaren magnetischen Feldes und somit die Zyklotronfrequenzen der Ionen ab. Diese Tatsache ermöglicht es Wellen mit immer niedrigerer Frequenz mit den Ionen resonant zu wechselwirken. Zusätzlich zu diesem Mechanismus muß ein Transfer spektraler Energiedichte der Wellen von großen zu kleinen Skalen berücksichtigt werden, der eine Folge der Nichtlinearität der Wechselwirkung zwischen den Wellen und dem Plasma ist. Die quasi-lineare Näherung beinhaltet diesen Effekt nicht. Für den Sonnenwind ist die genaue Form dieser direkten Kaskade unbekannt. Tu et al. (1984) schlugen ein vereinfachtes Modell des Transfers vor, welches in vielen Arbeiten Anwendung fand (Tu 1987, Hu et al. 1997, Li et al. 1997, 1999, Hu und Habbal 1999, Hu et al. 1999, 2000). Allerdings rief ihr Modell auch Widerspruch hervor (s. dazu Tu und Marsch 1995). Modelle, die sich des Sättigungsprinzips bedienen, beinhalten den spektralen Transfer letztlich implizit. Auch die Beschleunigung der Ionen wurde von Marsch et al. (1982a) mit Hilfe der quasi-linearen Theorie kinetisch berechnet. Andere Arbeiten verwendeten stattdessen eine auf ein mehrkomponentiges Plasma verallgemeinerte Beschleunigung der Ionen durch den Wellendruck (Goodrich 1978, McKenzie et al. 1979, Isenberg und Hollweg 1982). Sowohl die bevorzugte Beschleunigung der $\alpha$-Teilchen als auch ihre bevorzugte Heizung konnten bis heute nicht befriedigend modelliert werden. 


\subsection{Quasi-lineare Diffusion und Mikroinstabilitäten im Sonnenwind}

Aus der quasi-lineare Näherung der Welle-Teilchenwechselwirkung folgt die Diffusion der Teilchen des Plasmas im Geschwindigkeitsraum (Vedenov et al. 1962, Drummond und Pines 1962, Kennel und Engelmann 1966). Diese sogenannte quasi-lineare Diffusion führt unter geeigneten Umständen zur Ausbildung von Diffusionsplateaus in den Geschwindigkeitsverteilungen der Teilchen. Die Diffusionsplateaus sind in der Regel nicht isotrop, sondern können sich für Zyklotronwellen in der Richtung senkrecht zum magnetischen Feld zu größeren Geschwindigkeiten ausdehnen als parallel dazu. Dadurch drücken sie die Eigenschaft der quasi-linearen Diffusion aus, die Ionen bevorzugt in dieser Richtung heizen zu können (Arunasalam 1976). Die quasi-lineare Diffusion wird für die beobachtete Temperaturanisotropie im Kern der Geschwindigkeitsverteilung der Protonen verantwortlich gemacht. Die Protonen sollen dafür mit resonanten Alfvén-Wellen im Bereich der Zyklotronfrequenzen der Ionen wechselwirken. Von diesen Wellen wird angenommen, daß sie von der Sonne stammen und entlang des interplanetaren magnetischen Feldes auswärts laufen. Sie stehen vermutlich über eine direkte Kaskade in einem Zusammenhang mit den von Belcher und Davis (1971) beobachteten Alfvén-Wellen, die eine deutlich kleinere Frequenz besitzen. Es wurde ein Modell des Sonnenwindes aufgestellt, wonach die Geschwindigkeitsverteilung der Protonen die Plateaueigenschaft in jeder Entfernung zur Sonne exakt erfüllt (Isenberg 2001a,b,c). Dadurch sollte nicht nur die Temperaturanisotropie der Protonen sondern auch die Beschleunigung des schnellen Sonnenwindes erklärt werden. Marsch und Tu (2001) und Tu und Marsch (2002) untersuchten, ob sich im schnellen Sonnenwind tatsächlich Diffusionsplateaus in den Geschwindigkeitsverteilungen der Protonen beobachten lassen. Sie verglichen graphisch Höhenlinien einiger weniger Verteilungen mit theoretischen Diffusionsplateaus, wofür sie vereinfachende Annahmen über die Alfvén-Wellen machten, welche für die Temperaturanisotropie des Kerns der Geschwindigkeitsverteilungen der Protonen verantwortlich sein sollen. Sie stellten eine gute Übereinstimmung fest und sahen darin den Beweis der Gültigkeit der quasi-linearen Diffusion und der Voraussetzungen der quasi-linearen Näherung. Dazu zählt insbesondere die Annahme schwacher Turbulenz. Desweiteren sei die festgestellte Übereinstimmung der Höhenlinien und der theoretischen Plateaus ein Beweis für die Existenz der resonanten Alfvén-Wellen im Bereich der Zyklotronfrequenzen der Ionen. Die Diffusion der Protonen durch die Wechselwirkung mit Alfvén-Wellen bestimmt also die Form des Kern der Geschwindigkeitsverteilung der Protonen. Es wurde ebenfalls versucht die Form des Protonenstrahls durch die quasi-lineare Diffusion zu erklären, wofür Tu et al. (2002) nach Zyklotronwellen mit geeigneter Dispersion suchten. Sie stellten die Vermutung auf, daß der Protonenstrahl erst durch die quasi-lineare Diffusion entsteht.

Die im Sonnenwind vorhandenen Wellen müssen nicht vollständig von der Sonne stammen. Die beobachtete Turbulenz des Sonnenwindes kann zum Teil auch dort entstehen, wofür als Ursache Instabilitäten in Betracht kommen. In einem Plasma, in dem die Teilchen Temperaturanisotropien, nicht dieselbe mittlere Geschwindigkeit oder nicht dieselbe Temperatur besitzen, können Wellen linear instabil sein. Diese Instabilitäten ergeben sich aus der kinetischen Beschreibung eines kollisionsfreien Plasmas (Davidson 1983, Stix 1992, Brambilla 1998). Instabile Wellenmoden besitzen Frequenzen und Wellenlängen, 
die von der Größenordnung der Zyklotronfrequenzen bzw. der Lamorradien der Teilchen sind. Im Sonnenwind lösen möglicherweise die Temperaturanisotropie des Kerns der Geschwindigkeitsverteilung der Protonen und der Protonenstrahl Instabilitäten aus. Neben Mikroinstabilitäten, die sich aus den lokalen Eigenschaften der Geschwindigkeitsverteilungen der Teilchen ergeben, können im Sonnenwind auch Makroinstabilitäten auftreten, so z.B. an der Grenze von Strömungen des Sonnenwindes mit unterschiedlicher Geschwindigkeit. In dieser Arbeit werden nur Mikroinstabilitäten thematisiert. Durch die Energieerhaltung ist dem Anwachsen der Fluktuationen eines Plasmas aufgrund einer linearen Instabilität ein zeitliches Ende gesetzt. Eine Instabilität muß relaxieren und die lineare Anwachsrate muß sich unter eine Stabilitätsgrenze bewegen, auf der sie idealerweise verschwindet. Die Relaxation der Instabilitäten, die durch einen Protonenstrahl ausgelöst werden, leisten möglicherweise neben den Alfvén-Wellen im Bereich der Zyklotronfrequenzen der Ionen einen Beitrag zur Heizung der Protonen. Im Verlauf dieser Instabilitäten können die Temperaturanisotropien des Strahls und des Kerns der Geschwindigkeitsverteilung der Protonen erhöht werden (Gary et al. 1986, Daughton et al. 1999). Das Bild, wonach Strahl-Instabilitäten die kinetische Energie aus der Relativbewegung des Strahls zum Teil in thermische Energie senkrecht zum magnetischen Feld umwandeln, wurde bereits von Schwartz et al. (1981) vorgeschlagen. Die Relaxation einer linearen Instabilität läßt sich durch die quasi-lineare Näherung der Welle-TeilchenWechselwirkung beschreiben (s. z.B. Davidson und Ogden 1975). Auch in diesem Fall diffundieren die Teilchen durch ihre Wechselwirkung mit den instabilen Wellenmoden im Geschwindigkeitsraum. Nur im quasi-linearen Rahmen ist das Prinzip der marginalen Stabilität streng gültig: die lineare Anwachsrate verschwindet am Ende der Relaxation. Diese Grenze linearer Stabilität ist eine Zwangsbedingung für Plasmaparameter wie z.B. der Relativgeschwindigkeit des Strahls oder der Temperaturanisotropie der Protonen im Kern der Verteilung. Es ist unbekannt, ob das Prinzip der marginalen Stabilität im Sonnenwind tatsächlich gilt.

Die Arten von Mikroinstabilitäten, die im Sonnenwind auftreten können, sind aus Parameterstudien bekannt. In solchen Studien werden die Geschwindigkeitsverteilungen von Teilchenpopulationen durch Bi-Maxwell-Verteilungen modelliert. Montgomery et al. (1975), Montgomery et al. (1976) und Daughton und Gary (1998) untersuchten das Auftreten von Instabilitäten, die durch den Protonenstrahl verursacht werden. (Davidson und Ogden 1975) betrachteten die von einer Temperaturanisotropie ausgelöste Instabilität. Auf die Instabilitätstypen möchte ich im Kapitel 2 über die theoretische Grundlagen dieser Arbeit zu sprechen kommen. Das Auftreten dieser Instabilitäten wurde im Sonnenwind nur in den Arbeiten von Dum et al. (1980) und Leubner und Viñas (1986) durch die Berechnung von Anwachsraten direkt untersucht und dort wurden auch nur wenige Einzelfälle betrachtet. Im Unterschied dazu untersuchten Marsch und Livi (1987) und Goldstein et al. (2000) gemessene Geschwindigkeitsverteilungen indirekt auf Instabiltäten, wie sie vom Protonenstrahl ausgelöst werden, indem sie entscheidende Parameter der Verteilungen mit Stabilitätsgrenzen aus Parameterstudien verglichen (Gary et al. 1985, Daughton und Gary 1998). Im Widerspruch zum Prinzip der marginalen Stabilität fanden Marsch und Livi (1987) im Mittel instabile Verteilungen, die weit um die Stabilitätsgrenze streuten. Goldstein et al. (2000) kamen zu dem entgegengesetzten Ergebnis. Sie fanden deutlich unter diesen Grenzen liegende Parameter der Verteilungen. 


\subsection{Zielsetzungen und Aufbau der Arbeit}

Es ist die erste Zielsetzung dieser Arbeit, den schnellen Sonnenwind direkt auf lineare Instabilitäten zu untersuchen. Dafür verwende ich Geschwindigkeitsverteilungen der Protonen, der $\alpha$-Teilchen und der Elektronen, die vom Plasmaexperiment auf Helios 2 im solaren Minimum um das Jahres 1976 gemessen wurden. Die untersuchte Datenmenge deckt die Primärphase von Helios 2 möglichst vollständig ab. Aus jeder in dieser Phase von Helios 2 durchquerten Strömung schnellen Sonnenwindes wurde ein Zeitintervall von einem Tag herausgegriffen. Es wird ein Umfang von mehr als Tausend Datensätzen in jeder Strömung erzielt, wodurch die Ergebnisse der Untersuchungen dieser Arbeit auf sicherer statistischer Grundlage stehen. Die ausgewerteten Datenausschnitte liegen in dem Entfernungsbereich zwischen dem Perihel und dem Aphel von Helios 2 bei 0.29 AE bzw. $1 \mathrm{AE}$ beinahe äquidistant. Die linearen Anwachsraten der Instabilitäten hängen empfindlich von der Form der Geschwindigkeitsverteilungen ab (Dum et al. 1980). Deshalb wurde ein Modell für die gemessenen Verteilungen aufgestellt, welches die vom Meßinstrument erzielte Auflösung im Geschwindigkeitsraum so weit als möglich ausnutzt und dennoch die Berechnung der linearer Anwachsraten zuläßt. Ich verwende nur dann Bi-MaxwellVerteilungen, wenn es aus praktischen Gründen unvermeidbar ist. Ich beabsichtige aufzuklären, welche Mikroinstabilitäten tatsächlich auftreten und ob ihre Anwachsraten ggf. marginal stabil sind. Diese Ergebnisse erlauben wichtige Rückschlüsse auf den turbulenten Zustand des schnellen Sonnenwindes. Durch den Nachweis einer linearen Instabilität darf geschlußfolgert werden, daß die betreffenden instabilen Moden angeregt sind. Sie wechselwirken unter Umständen quasi-linear mit den Teilchen des Sonnenwindes und beeinflussen möglicherweise entscheidend deren Geschwindigkeitsverteilungen (s. z.B. Schwartz et al. 1981), weshalb sie in Modellen berücksichtigt werden müssen. Im Sonnenwind ist das Wellenspektrum nur unzureichend durch direkte Messungen bekannt. Auf diese Weise kann nicht gesagt werden, welche von den Moden des Plasmas, die mit den Teilchen resonant wechselwirken können, auch angeregt sind. Die Beobachtung einer Abweichung von der marginalen Stabilität hat ebenfalls wichtige Konsequenzen. Sie zeigt an, daß die betreffenden Moden einer nichtlinearen Wechselwirkung ausgesetzt sind, die nicht vernachlässigt werden darf. Nach allen Erkenntnisse ist der Zustand des schnellen Sonnenwindes stationär und die tatsächlichen Anwachsraten der betreffenden Moden müssen marginal sein. Diese setzen sich aus der linearen Rate und nichtlinearen Korrekturen zusammen. Letztere können nach der Beobachtung nicht verschwinden. Sie sind sowohl die Konsequenz nichtlinearer Welle-Welle-Wechselwirkung (s. z.B. Galeev und Sagdeev 1983b) als auch nichtlinearer Welle-Teilchen-Wechselwirkung (s. z.B. Galeev und Sagdeev 1983c). Im ersten Fall zerfallen die betreffenden Wellenmoden in andere Moden, und im zweiten Fall sind sie nichtlinear gedämpft. Es ist die zweite Zielsetzung dieser Arbeit, die Geschwindigkeitsverteilungen der Protonen im schnellen Sonnenwind auf quasi-lineare Diffusionsplateaus zu untersuchen. Die für diesen Bestandteils der Arbeit ausgewertete Datenmenge stimmt mit der zuvor genannten überein. Es soll herausgefunden werden, welche Wellenmoden ggf. für die Ausbildung der Diffusionsplateaus verantwortlich sind. Die von der Sonne auswärts laufenden Alfvén-Wellen im Bereich der Zyklotronfrequenzen der Ionen stehen dabei im Mittelpunkt. Der Nachweis der durch sie verursachten theoretischen Diffusionsplateaus im Kern der Verteilungen der Protonen bestätigt ihre Existenz und zeigt, daß sie die Ursache für die Temperaturanisotropie 
des Kerns sind. Aus der Beobachtung von quasi-linearen Diffusionsplateaus in gemessenen Geschwindigkeitsverteilungen darf geschlußfolgert werden, daß die quasi-lineare Näherung im schnellen Sonnenwind weitgehend zutreffend ist. Die Annahmen schwacher Turbulenz und der Trennung von turbulenten und mittleren Skalen sind in diesem Fall gerechtfertigt (s. Kapitel 2). Zusammenfassend unternehme ich mit dieser Arbeit den Versuch, die im schnellen Sonnenwind relevanten kinetischen Wechselwirkungsprozesse zwischen Wellen und Teilchen soweit aufzuklären, wie es die bloße Messung von Geschwindigeitsverteilungen erlaubt.

Im Kapitel 2 sollen die Grundlagen der Beschreibung kinetischer Plasmawellen und der quasi-linearen Diffusion dargestellt werden. Insbesondere möchte ich die aus Parameterstudien bekannten Instabilitäten ansprechen und das Zustandekommen von Diffusionsplateaus erklären. Im Kapitel 3 werden die Helios Mission und die Instrumente des Plasmaexperimentes auf Helios beschrieben, von denen die in dieser Arbeit untersuchten Meßdaten stammen. Ich gehe darauf ein, wie sich Meßwerte der Geschwindigkeitsverteilungen der $\alpha$-Teilchen bestimmen lassen. Ich schildere, mit welchen besonderen Schwierigkeiten die Auswertung der Messungen der Elektronen verbunden ist, und wie ich damit umgegangen bin. Abschließend erläutere ich die Datenauswahl, die ich getroffen habe. Im Kapitel 4 wird die Modellierung der Geschwindigkeitsverteilungen der Protonen aus den vorhandenen Meßwerten vorgestellt und die Methode beschrieben, durch die sie sich im Einzelfall numerisch gewinnen läßt. Es handelt sich um eine in diesem Zusammenhang bisher nicht gebrauchte Darstellungsform, die den Erfordernissen dieser Arbeit besonders entspricht. Im Kapitel 5 wird die numerische Methode beschrieben, mit der in dieser Arbeit die Dispersionsbeziehungen kinetischer Plasmawellen gelöst und die Abhängigkeit der Moden maximaler Anwachsrate von Modellparametern bestimmt wird. Im Kapitel 6 präsentiere ich die Ergebnisse der mit den Meßdaten des Plasmaexperimentes durchgeführten Untersuchung des schnellen Sonnenwindes auf Instabilitäten kinetischer Plasmawellen. Dabei behandle ich die Instabilitäten von sich schräg und parallel zum magnetischen Feld ausbreitenden Moden in den einzelnen Abschnitten 6.3 bzw. 6.4. Im Kapitel 7 stelle ich die Ergebnisse der Untersuchung der gemessenen Geschwindigkeitsverteilungen der Protonen auf die Ausbildung von Diffusionsplateaus vor. 



\section{Grundlagen}

In dieser Arbeit habe ich den schnellen Sonnenwind anhand der vom Plasmaexperiment auf Helios gemessenen Geschwindigkeitsverteilungen der Protonen, $\alpha$-Teilchen und Elektronen auf Mikroinstabilitäten untersucht. Desweiteren wurde die von der quasilineare Näherung der Welle-Teilchen-Wechselwirkung gemachte Vorhersage von Diffusionsplateaus in den Geschwindigkeitsverteilungen der Protonen überprüft. Es galt herauszufinden, ob sich die Form des Kerns der Geschwindigkeitsverteilung der Protonen und die Form des beobachteten Protonenstrahls durch Diffusionsplateaus erklären lassen. Das Ergebnis dieser Untersuchung erlaubt zudem Rückschlüsse auf die Gültigkeit der quasi-linearen Näherung und ihrer Voraussetzungen im schnellen Sonnenwind. In diesem Kapitel sollen die Grundlagen kinetischer Plasmaphysik beschrieben werden, auf denen diese Arbeit beruht.

\subsection{Theorie mittlerer Felder}

Der Ausgangspunkt der kinetischen Beschreibung eines Plasmas sind die KlimontovichMaxwell-Gleichungen für das elektromagnetische Feld und die Mikrodichten $f_{s}(\vec{x}, \vec{v}, t)$ der Teilchenspezies des Plasmas

$$
\begin{gathered}
\vec{\nabla} \times \vec{B}=\frac{4 \pi}{c} \sum_{s} e_{s} \int d^{3} v \vec{v} f_{s}+\frac{1}{c} \frac{\partial \vec{E}}{\partial t}, \\
\vec{\nabla} \times \vec{E}=-\frac{1}{c} \frac{\partial \vec{B}}{\partial t}, \\
\nabla \cdot \vec{B}=0, \quad \nabla \cdot \vec{E}=4 \pi \sum_{s} e_{s} \int d^{3} v f_{s}, \\
\frac{\partial f_{s}}{\partial t}+\vec{v} \cdot \vec{\nabla} f_{s}+\frac{e_{s}}{m_{s}}\left(\vec{E}+\frac{\vec{v}}{c} \times \vec{B}\right) \cdot \frac{\partial f_{s}}{\partial \vec{v}}=0 .
\end{gathered}
$$

Die Mikrodichten sind durch die Orte und Geschwindigkeiten der einzelnen Teilchen des Plasmas definiert

$$
f_{s}(\vec{x}, \vec{v}, t)=\sum_{i=1}^{N_{s}} \delta\left(\vec{x}-\vec{x}_{s, i}(t)\right) \delta\left(\vec{v}-\vec{v}_{s, i}(t)\right)
$$


welche in der notwendigen statistischen Beschreibung des Plasmas zusammen mit dem elektrischen und magnetischen Feld als Zufallsgrößen anzusehen sind. Das Mittel einer Zufallsgröße sei durch $\langle\cdot\rangle$ bezeichnet. Die Ein-Teilchen-Verteilungsfunktionen $\left\langle f_{s}\right\rangle$ werden in dieser Arbeit schlicht Geschwindigkeitsverteilungen genannt. Die Bewegungsgleichungen der Geschwindigkeitsverteilungen lassen sich durch die Mittelung der Klimontovich-Gleichung bestimmen. Die Zerlegung nach mittleren und fluktuierenden Größen

$$
f_{s}=\delta f_{s}+\left\langle f_{s}\right\rangle, \quad \vec{B}=\delta \vec{B}+\langle\vec{B}\rangle, \quad \vec{E}=\delta \vec{E}+\langle\vec{E}\rangle
$$

führt zu

$$
\frac{\partial\left\langle f_{s}\right\rangle}{\partial t}+\vec{v} \cdot \vec{\nabla}\left\langle f_{s}\right\rangle+\frac{e_{s}}{m_{s}}\left(\langle\vec{E}\rangle+\frac{\vec{v}}{c} \times\langle\vec{B}\rangle\right) \cdot \frac{\partial\left\langle f_{s}\right\rangle}{\partial \vec{v}}=-\left\langle\delta \vec{a}_{s} \cdot \frac{\partial \delta f_{s}}{\partial \vec{v}}\right\rangle .
$$

Die fluktuierenden Anteile der Mikrodichten erfüllen

$$
\begin{aligned}
\frac{\partial \delta f_{s}}{\partial t}+\vec{v} \cdot \vec{\nabla} \delta f_{s} & +\frac{e_{s}}{m_{s}}\left(\langle\vec{E}\rangle+\frac{\vec{v}}{c} \times\langle\vec{B}\rangle\right) \cdot \frac{\partial \delta f_{s}}{\partial \vec{v}}=-\delta \vec{a}_{s} \cdot \frac{\partial\left\langle f_{s}\right\rangle}{\partial \vec{v}} \\
& +\left\langle\delta \vec{a}_{s} \cdot \frac{\partial \delta f_{s}}{\partial \vec{v}}\right\rangle-\delta \vec{a}_{s} \cdot \frac{\partial \delta f_{s}}{\partial \vec{v}}
\end{aligned}
$$

worin abkürzend die Lorentz-Beschleunigungen durch die fluktuierenden elektromagnetischen Felder

$$
\delta \vec{a}_{s}=\frac{e_{s}}{m_{s}}\left(\delta \vec{E}+\frac{\vec{v}}{c} \times \delta \vec{B}\right)
$$

auftauchen ${ }^{1}$. Plasmawellen dürfen als Bestandteil der fluktuierenden Größen betrachtet werden. Im Abschnitt 2.2 soll die Dispersionsbeziehung von Plasmawellen vor dem Hintergrund homogener und stationärer mittlerer Felder vorgestellt werden. Es werden insbesondere diejenigen Dispersionszweige, deren Moden für bestimmte Geschwindigkeitsverteilungen der Teilchen des Plasmas instabil sind, diskutiert. Im Sonnenwind können zwei Kategorien solcher Mikroinstabilitäten auftreten. Im Abschnitt 2.3 wird die quasilinearen Näherung der Wechselwirkung von Plasmawellen mit den Teilchen des Plasmas beschrieben. Diese Wechselwirkung drückt sich durch den Mittelwert auf der rechten Seite der Gleichung 2.7 der Geschwindigkeitsverteilungen aus. Im Rahmen der quasilinearen Näherung ist es möglich, den Wechselwirkungsterm durch die 2-Punkt-Korrelationen der fluktuierenden elektromagnetischen Felder und die Geschwindigkeitsverteilungen selbst auszudrücken. Es ergibt sich die sogenannte quasi-lineare Diffusion, deren Konsequenz unter geeigneten Voraussetzungen die Ausbildung von Diffusionsplateaus in den Geschwindigkeitsverteilungen ist.

\subsection{Kinetische Plasmawellen und ihre Instabilitäten}

Die für die fluktuierenden Felder linearisierten Gleichungen 2.8 lassen sich unter der Annahme homogener und stationärer mittlerer Felder durch eine Fourier-Laplace-Transfor-

\footnotetext{
${ }^{1}$ Produkte von Feldern am gleichen Ort sind nicht definiert, sondern z.B. erst $f_{s_{1} s_{2}}(1,2)=$ $\left\langle f_{s_{1}}(1) f_{s_{2}}(2)\right\rangle-\delta_{s_{1} s_{2}} \delta(1-2)\left\langle f_{s_{1}}(1)\right\rangle$. Diese Tatsache ist in der Gleichung 2.4 nicht sichtbar und soll vernachlässigt werden.
} 
mation lösen. Die Vernachlässigung der nicht-linearen Terme kann durch kleine Amplituden der Fluktuationen, d.h. die Vorstellung schwacher Turbulenz des Plasmas begründet werden. Es wird im allgemeinen vereinfachend vorausgesetzt, daß die Geschwindigkeitsverteilungen der Teilchen symmetrisch um das mittlere magnetische Feld sind. Diese Symmetrie bleibt z.B. durch die quasi-lineare Welle-Teilchen-Wechselwirkung erhalten und wird im schnellen und langsamen Sonnenwind durch Beobachtungen gestützt. Das mittlere elektrische Feld muß verschwinden, was zumindest für die Komponente senkrecht zum mittleren magnetischen Feld durch einen Wechsel des Bezugssystems erreicht werden kann ${ }^{2}$. Die Lösung für die Fluktuationen des elektrischen Feldes besitzt damit die allgemeine Form

$$
\mathbf{D}(\omega, \vec{k}) \cdot \delta \vec{E}(\omega, \vec{k})=\delta \vec{A}(\omega, \vec{k})
$$

wobei die rechte Seite dieser Gleichung von den Anfangsbedingungen der fluktuierenden Felder abhängt. Die Dispersionmatrix D gibt durch die Stellen in der komplexen $\omega$ Ebene, an denen sie bei reellem Wellenvektor singulär wird, die Moden des Plasmas vor. Die Abhängigkeit der Dispersionsmatrix von der Frequenz ist in der unteren komplexen Halbebene durch analytische Fortsetzung definiert. Ohne Einschränkung der Allgemeinheit soll die Richtung des mittleren magnetischen Feldes im folgenden mit dem Basisrichtung $\vec{e}_{3}$ zusammen fallen. Die Dispersionsmatrix besitzt damit die generelle Form

$$
\mathbf{D}(\omega, \vec{k})=\omega^{2}+c^{2}\left(\vec{k} \vec{k}-k^{2}\right)+\sum_{s} \frac{\omega_{p s}^{2}}{n_{s}} \sum_{n=-\infty}^{\infty} \int d^{3} v \frac{\widehat{\mathbf{S}}_{n}^{s}\left\langle f_{s}\right\rangle}{\omega-k_{\|} v_{\|}-n \Omega_{s}}
$$

(s. z.B. Stix 1992, Brambilla 1998), wobei die Abkürzungen

$$
\begin{gathered}
\widehat{\mathbf{S}}_{n}^{s}=\left(\begin{array}{ccc}
v_{\perp} \frac{n^{2} J_{n}^{2}}{a_{s}^{2}} \widehat{U} & i v_{\perp} \frac{n J_{n} J_{n}^{\prime}}{a_{s}} \widehat{U} & v_{\|} \frac{n J_{n}^{2}}{a_{s}} \widehat{U} \\
-i v_{\perp} \frac{n J_{n} J_{n}^{\prime}}{a_{s}} \widehat{U} & v_{\perp} J_{n}^{\prime 2} \widehat{U} & -i v_{\|} J_{n} J_{n}^{\prime} \widehat{U} \\
v_{\|} \frac{n J_{n}^{2}}{a_{s}} \widehat{U} & i v_{\|} J_{n} J_{n}^{\prime} \widehat{U} & v_{\|} J_{n}^{2} \widehat{W}
\end{array}\right) \\
\widehat{U}=\omega \frac{\partial}{\partial v_{\perp}}-k_{\|}\left(v_{\|} \frac{\partial}{\partial v_{\perp}}-v_{\perp} \frac{\partial}{\partial v_{\|}}\right) \\
\widehat{W}=\omega \frac{\partial}{\partial v_{\|}}+\frac{n \Omega_{s}}{v_{\perp}}\left(v_{\|} \frac{\partial}{\partial v_{\perp}}-v_{\perp} \frac{\partial}{\partial v_{\|}}\right)
\end{gathered}
$$

und

$$
J_{n}=J_{n}\left(a_{s}\right), \quad a_{s}=\frac{k_{\perp} v_{\perp}}{\Omega_{s}}
$$

verwendet werden und $J_{n}$ die Bessel-Funktionen erster Art bezeichnen.

\subsubsection{Dispersion bei paralleler Ausbreitungsrichtung}

Für sich parallel zum mittleren magnetischen ausbreitende Feld Wellenmoden $\vec{k}=k_{\|} \vec{e}_{3}$ nimmt die Dispersionsmatrix eine besonders einfache Form an, da in diesem Fall $J_{n}=$

\footnotetext{
${ }^{2}$ Man verwende das mit der Drift-Geschwindigkeit $c(\langle\vec{E}\rangle \times\langle\vec{B}\rangle) /\langle\vec{B}\rangle^{2}$ bewegte Bezugssystem.
} 


\begin{tabular}{ccc}
\hline \hline Parameter & Protonen & Elektronen \\
\hline$n / n_{p}$ & 1 & 1 \\
$T_{\|} / T_{\|, p}$ & 1 & 1 \\
$T_{\perp} / T_{\|}$ & 1 & 1 \\
\hline \hline
\end{tabular}

Tabelle 2.1: Exemplarische Parameter eines Protonen/Elektronen-Plasmas. Im übrigen sind $v_{A} / c=10^{-4}$ und $\beta_{\|, p}=0.3$.

$\delta_{n, 0}$ gilt. Es ergibt sich

$$
\mathbf{D}\left(\omega, k_{\|}\right)=\left(\begin{array}{ccc}
\left(D_{+}+D_{-}\right) / 2 & -i\left(D_{+}-D_{-}\right) / 2 & 0 \\
i\left(D_{+}-D_{-}\right) / 2 & \left(D_{+}+D_{-}\right) / 2 & 0 \\
0 & 0 & D_{\|}\left(\omega, k_{\|}\right)
\end{array}\right)
$$

mit den Dispersionsfunktionen

$$
D_{\|}\left(\omega, k_{\|}\right)=\omega^{2}+\sum_{s} \frac{\omega_{p s}^{2}}{n_{s}} \int d v_{\|} \frac{\omega v_{\|}}{\omega-k_{\|} v_{\|}} \frac{\partial F_{s, 0}}{\partial v_{\|}}
$$

und

$$
D_{ \pm}\left(\omega, k_{\|}\right)=\omega^{2}-c^{2} k_{\|}^{2}+\sum_{s} \frac{\omega_{p s}^{2}}{n_{s}} \int d v_{\|} \frac{k_{\|} \partial F_{s, 1} / \partial v_{\|}-2\left(\omega-k_{\|} v_{\|}\right) F_{s, 0}}{2\left(\omega-k_{\|} v_{\|} \pm \Omega_{s}\right)}
$$

die sich durch die reduzierten Geschwindigkeitsverteilungen

$$
F_{s, n}\left(v_{\|}\right)=2 \pi \int_{0}^{\infty} d v_{\perp} v_{\perp}^{2 n+1}\left\langle f_{s}\right\rangle\left(v_{\|}, v_{\perp}\right)
$$

nullter und erster Ordnung ausdrücken. Die Dispersionsmatrix ist diagonal in den Vektoren $\vec{e}_{ \pm}=\left(\vec{e}_{1} \pm i \vec{e}_{2}\right) / \sqrt{2}$ und $\vec{e}_{3}$, die somit die Polarisationsvektoren der sich parallel zum mittleren magnetischen Feld ausbreitenden Zyklotronmoden

$$
D_{ \pm}\left(\omega, k_{\|}\right)=0
$$

bzw. elekrostatischen Moden

$$
D_{\|}\left(\omega, k_{\|}\right)=0
$$

des Plasmas sind. Die Zyklotronmoden sind rechts- bzw. links-zirkular polarisiert. Für die parallel zur eigenen Ausbreitungsrichtung polarisierten elektrostatischen Moden verschwindet der magnetische Feldvektor. Sie sind mit Fluktuationen der elektrischen Ladungsdichte verknüpft. 


\begin{tabular}{cccc}
\hline \hline Parameter & Protonen-Kern & Protonen-Strahl & Elektronen \\
\hline$n / n_{p}$ & 0.75 & 0.25 & 1 \\
$T_{\|} / T_{\|, k}$ & 1 & 1 & 1 \\
$T_{\perp} / T_{\|}$ & 1 & 1 & 1 \\
\hline \hline
\end{tabular}

Tabelle 2.2: Parameter eines Protonen/Elektronen-Plasmas mit Strahl-Instabilitäten. Im übrigen sind $v_{A} / c=10^{-4}, \beta_{\|, k}=0.3$ und die Relativgeschwindigkeit des ProtonenStrahls $\Delta v_{k, s}=1.7 v_{A}$.

\subsubsection{Dispersion für Bi-Maxwell-Verteilungen}

Die Einschränkung auf sich parallel zum mittleren magnetischen Feld ausbreitende Wellenmoden zeigt, daß alle Moden entweder elektrodynamisch und zirkular polarisiert oder aber elektrostatisch sind. Die Dispersion von Wellenmoden kann allerding nur unter der Vorgabe spezifischer Geschwindigkeitsverteilungen diskutiert werden. Ein einfaches Modell einer Verteilung, das auch die im Sonnenwind beobachteten Temperaturanisotropien relativ zum mittleren magnetischen Feld zuläßt, ist die Bi-Maxwell-Verteilung

$$
\left\langle f_{s}\right\rangle\left(v_{\|}, v_{\perp}\right)=n_{s} \frac{(2 \pi)^{-3 / 2}}{V_{\|, s} V_{\perp, s}^{2}} e^{-\left(v_{\|}-v_{\|, s}\right)^{2} / 2 V_{\|, s}^{2}} e^{-v_{\perp}^{2} / 2 V_{\perp, s}^{2}}
$$

mit einer mittleren Geschwindigkeit parallel zum magnetischen Feld $v_{\|, s}$, den thermischen Geschwindigkeiten $V_{\|, s}^{2}=m_{s} / k_{B} T_{\|, s}, V_{\perp, s}^{2}=m_{s} / k_{B} T_{\perp, s}$ sowie den Temperaturen $T_{\|, s}, T_{\perp, s}$ parallel bzw. senkrecht zum mittleren magnetischen Feld. Die Annahme, daß alle Geschwindigkeitsverteilungen diese Form besitzen, erlaubt es, die Dispersionsmatrix analytisch zu berechnen. Im Kapitel 4 wird ein allgemeineres Modell von Verteilungsfunktionen diskutiert, welches diese Berechnung auch zuläßt. Es wird in dieser Arbeit zur Darstellung der gemessenen Geschwindigkeitsverteilungen der Protonen verwendet. Allerdings mache ich ebenfalls von der Bi-Maxwell-Verteilung Gebrauch. Alle bekannten Parameterstudien von Mikroinstabilitäten im Sonnenwind basieren auf dieser Verteilungsform. Die Dispersionsmatrix lautet in diesem Fall (Stix 1992)

$$
\mathbf{D}=\omega^{2}+c^{2}\left(\vec{k} \vec{k}-k^{2}\right)+\sum_{s} \omega_{p s}^{2}\left\{\frac{v_{\|, s} \omega}{V_{\perp, s}^{2} k_{\|}} \vec{e}_{3} \vec{e}_{3}+\sum_{n=-\infty}^{\infty} \mathbf{Y}_{n}^{s} e^{-\lambda_{s}}\right\}
$$

wobei abkürzende Symbole durch

$$
\begin{aligned}
& \mathbf{Y}_{n}^{s}= \\
& \left(\begin{array}{ccc}
\frac{n^{2} I_{n}}{\lambda_{s}} A_{s n} & i n\left(I_{n}^{\prime}-I_{n}\right) A_{s n} & \frac{n k_{\perp}}{\lambda_{s} \Omega_{s}} I_{n} B_{s n} \\
-i n\left(I_{n}^{\prime}-I_{n}\right) A_{s n} & \left(-2 \lambda_{s} I_{n}^{\prime}+2 \lambda_{s} I_{n}+\frac{n^{2}}{\lambda_{s}} I_{n}\right) A_{s n} & -i \frac{k_{\perp}}{\Omega_{s}}\left(I_{n}^{\prime}-I_{n}\right) B_{s n} \\
\frac{n k_{\perp}}{\lambda_{s} \Omega_{s}} I_{n} B_{s n} & i \frac{k_{\perp}}{\Omega_{s}}\left(I_{n}^{\prime}-I_{n}\right) B_{s n} & \frac{I_{n}\left(\omega-n \Omega_{s}\right)}{V_{\perp, s}^{2} k_{\|}} B_{s n}
\end{array}\right)
\end{aligned}
$$

und $\lambda_{s}=k_{\perp}^{2} V_{\perp, s}^{2} / \Omega_{s}^{2}$ definiert sind. Mit $I_{n}$ werden die modifizierten Bessel-Funktionen erster Art bezeichnet: $I_{n}=I_{n}\left(\lambda_{s}\right)=i^{-n} J_{n}\left(i \lambda_{s}\right)$. Es tauchen mit $\Omega_{s}=e_{s} B / \mathrm{cm}_{s}$ und 
(a)

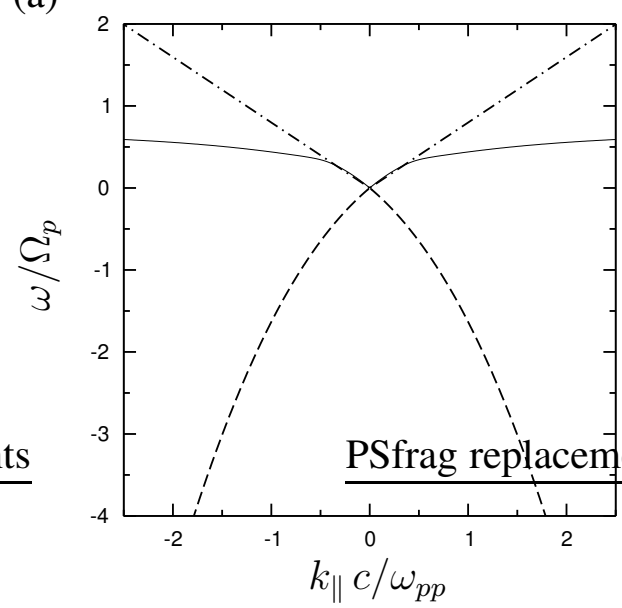

(b)

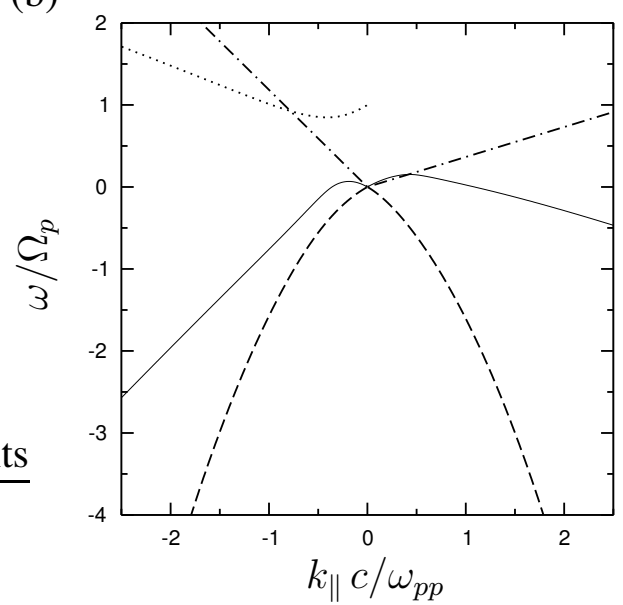

Abbildung 2.1: Es ist die Abhängigkeit der Frequenz der magnetosonischen (---), der ionen-akustischen (---) und der Alfvén-Dispersionszweige (-) des Plasmas mit (a) den Parametern der Tabelle 2.1 und (b) den Parametern der Tabelle 2.2 von der Wellenzahl bei der Ausbreitung parallel zum magnetischen Feld dargestellt. Die gepunktete Kurve in (b) zeigt den einzigen weiteren Zyklotron-Dispersionszweig des Plasmas, dessen Moden resonant mit dem Kern der Verteilung der Protonen sind.

$\omega_{p s}=4 \pi e_{s}^{2} n_{s} / m_{s}$ die Zyklotron- bzw. die Plasmafrequenz der Teilchensorte $s$ auf. Die Koeffizienten

$$
A_{s n}=\zeta_{s 0} Z\left(\zeta_{s n}\right)-\frac{1}{2}\left(\frac{T_{\perp, s}}{T_{\|, s}}-1\right) Z^{\prime}\left(\zeta_{s n}\right)
$$

und

$$
B_{s n}=v_{\|, s} \zeta_{s 0} Z\left(\zeta_{s n}\right)-\frac{1}{2}\left(\frac{\omega-k_{\|} v_{\|, s}}{k_{\|}}+\left(\frac{T_{\perp, s}}{T_{\|, s}}-1\right) \frac{\omega-n \Omega_{s}}{k_{\|}}\right) Z^{\prime}\left(\zeta_{s n}\right)
$$

mit

$$
\zeta_{s n}=\frac{1}{\sqrt{2} V_{\|, s}} \frac{\omega-k_{\|} v_{\|, s}-n \Omega_{s}}{k_{\|}}
$$

drücken sich durch die nicht-relativistische Plasmadispersionsfunktion (Fried und Conte 1961) $Z$ und ihre Ableitung $Z^{\prime}$ aus. Diese spezielle Funktion ist in der ganzen komplexen Ebene analytisch und durch

$$
Z(\zeta)=\frac{1}{\sqrt{\pi}} \int d t \frac{e^{-t^{2}}}{t-\zeta}, \quad \operatorname{Im} \zeta>0
$$

eindeutig definiert. Sie kann aber auch mit Hilfe der komplexen Fehlerfunkion (s. Abramowitz und Stegun 1965, S. 297ff.) geschrieben werden:

$$
Z(\zeta)=i \sqrt{\pi} e^{-\zeta^{2}}(1-\operatorname{erf}(-i \zeta))
$$

Aufgrund der Art, wie die Parameter der Bi-Maxwell-Verteilungen der Teilchen in die Dispersionsmaxtrix eingehen, ist es sinnvoll, im folgenden die normierten Dichten $n_{s} / n_{p}$, 
die auf die Alfvén-Geschwindigkeit $v_{A}=B / \sqrt{4 \pi m_{p} n_{p}}$ normierten mittleren Geschwindigkeiten $v_{\|, s} / v_{A}$, die Plasmabetas $\beta_{\|, s}=2 V_{\|, s}^{2} / v_{A}^{2}$ und $\beta_{\perp, s}=2 V_{\perp, s}^{2} / v_{A}^{2}$ sowie die Temperaturanisotropien $T_{\perp, s} / T_{\|, s}$ zu betrachten. Die Dispersionsbeziehung zwischen der normierten Frequenz $\omega / \Omega_{p}$ und dem normierten Wellenvektor $c \vec{k} / \omega_{p p}$ hängt nur von diesen entdimensionalisierten Parametern sowie von $v_{A} / c$ ab. Die mittleren Geschwindigkeiten der Teilchen und alle im verbleibenden Teil dieser Arbeit dargestellten Dispersionszweige beziehen sich stets auf das Massenschwerpunktssystem aller Teilchen, d.h. das Ruhesystem des Plasmas. Die Frequenz in anderen Bezugssystemen unterscheidet sich davon durch eine Dopplerverschiebung. Selbst wenn dies im Einzelfall nicht explizit erwähnt wird, sollen alle in dieser Arbeit betrachteten Plasmen im Mittel weder eine elektrische Ladungs- noch Stromdichte aufweisen. Die Verteilungen der Elektronen sind entsprechend angepaßt.

In den Abbildung 2.1a und 2.1b sind Dispersionszweige bei der Ausbreitung parallel zum mittleren magnetischen Feld im Bereich der Ionen-Zyklotronfrequenzen $\omega \lesssim \Omega_{p}$ für die exemplarischen Plasmen mit den in den Tabellen 2.1 und 2.2 angegebenen Parametern zu sehen. Das zweite Plasma enthält einen Protonenstrahl, der die Symmetrie der Dispersionszweige bezüglich des Vorzeichens der Wellenzahl $k_{\|}$bricht. Bei den gezeigten Zweigen handelt sich um die sogenannten magnetosonischen, ionen-akustischen und Alfvén-Zweige (Stix 1992, Brambilla 1998). Die magnetosonischen und die AlfvénModen sind Zyklotronwellen, während die ionen-akustischen Moden elektrostatisch sind. Diese Zweige sind in der gezeigten Form Lösungen der Dispersionsbeziehung über $D_{-}$. Mit einer Lösung $\omega$ der Dispersionsbeziehung zu $\vec{k}$ ist allerdings auch $-\omega^{*}$ eine Lösung $\mathrm{zu}-\vec{k}$, was bereits für die allgemeine Dispersionsmatrix 2.11 zutrifft. Die beiden durch diese Symmetrie verbundenen Moden sind aus mathematischen Gründen stets mit gleicher Amplitude angeregt. Für kleine Wellenzahlen und Frequenzen gehen die magnetosonischen und die Alfvén-Zweige in die gleichnamigen Zweige der idealen MHD über, was auch bei der Ausbreitung schräg zum mittleren magnetischen Feld gilt. Der Vollständigkeit halber muß erwähnt werden, daß die Dispersionsbeziehung zu jedem Wellenvektor prinzipiell unendlich viele Lösungen besitzt. Außer den in den Abbildungen gezeigten Moden, sind aber alle Moden im Bereich der Ionen-Zyklotronfrequenzen stark gedämpft und somit physikalisch unbedeutend. Dies gilt bereits für betraglich kleine Wellenvektoren, bei denen die magnetosonischen und die Alfvén-Moden ungedämpft sind. Sie sind es, die unter den im schnellen Sonnenwind gegebenen Bedingungen instabil sein können.

\subsubsection{Mikroinstabilitäten}

Mikroinstabilitäten werden durch Temperaturanisotropien und die Relativbewegung von Teilchenpopulationen verursacht. Im schnellen Sonnenwind kommen Instabilitäten aufgrund der Temperaturanisotropie des Kerns der Geschwindigkeitsverteilung der Protonen und aufgrund der Relativbewegung eines Protonenstrahls in Betracht. Die Verteilungen der $\alpha$-Teilchen und Elektronen beeinflussen die Anwachsraten. Unter den Gegebenheiten des schnellen Sonnenwindes verursachen sie jedoch selbst keine Instabilitäten. So sind z.B. die ionen-akustischen Moden nur dann instabil (s. z.B. Stix 1992, Brambilla 1998), wenn die Elektronentemperatur diejenige der Protonen in einem Maße übersteigt, wie es im Sonnenwind nicht zu beobachten ist. 
(a)

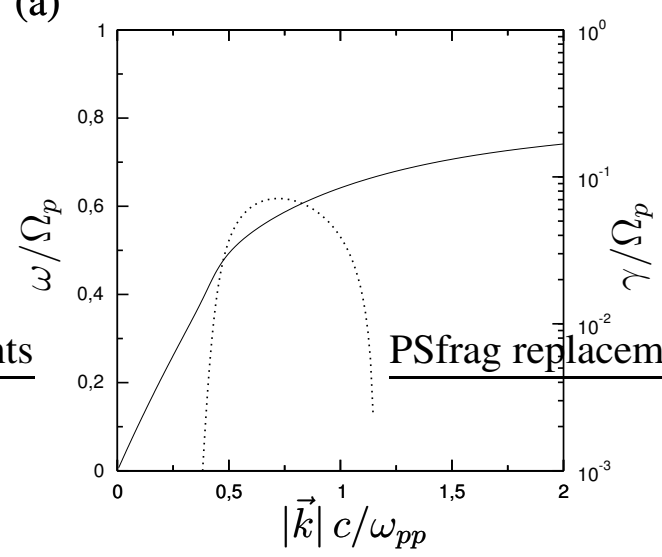

(b)

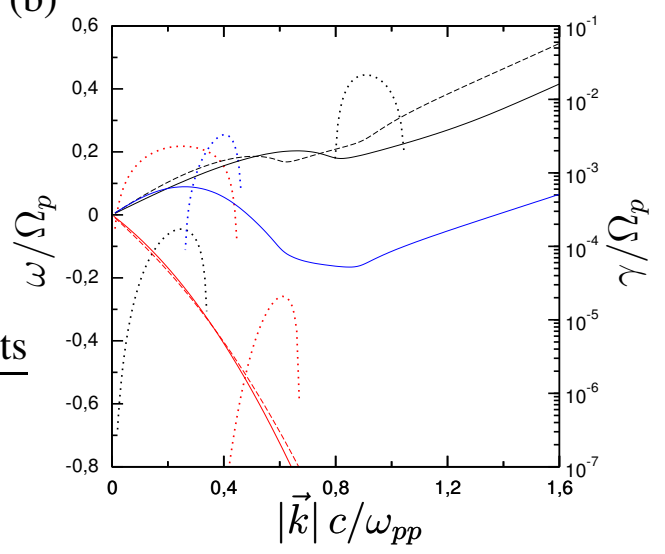

Abbildung 2.2: Verlauf der Dispersionszweige (a) der Temperaturanisotropie-Instabilität bei $\theta=0^{\circ}$ und (b) der Strahl-Instabilitäten jeweils entlang der Richtung maximaler Anwachsrate bei $\theta=50.2^{\circ}$ (Alfvén I, schwarz), $\theta=34.9^{\circ}$ (Alfvén II, schwarz gestrichelt), $\theta=146.6^{\circ}$ (Alfvén III, blau), $\theta=180^{\circ}$ (parallel magnetosonisch, rot) und $\theta=150.5^{\circ}$ (schräg magnetosonisch, rot gestrichelt). Der Verlauf der Anwachsraten ist in (a) und (b) durch die gepunkteten Kurven dargestellt. Es liegen (a) und (b) die Parameter der Tabelle 2.1 mit $T_{\perp, p} / T_{\|, p}=3$ bzw. der Tabelle 2.2 zugrunde.

Die durch eine Temperaturanisotropie der Protonen verursachte Mikroinstabilität betrifft ausschließlich die Alfvén-Moden (Davidson und Ogden 1975, Davidson 1983). Diese Instabilität hat ihre maximale Anwachsrate bei der Ausbreitung parallel zum mittleren magnetischen Feld. Solange keine Richtung entlang des magnetischen Feldes ausgezeichnet ist, sind ggf. sowohl die Alfvén-Moden bei positiver als auch bei negativer Wellenzahl $k_{\|}$in gleicher Weise instabil. Ein Protonenstrahl bricht diese Symmetrie (vgl. Abbildungen 2.1a und 2.1b). Die Abbildung 2.2a zeigt den Verlauf der Anwachsrate der Anisotropie-Instabilität für das Plasma mit den Parametern der Tabelle 2.1 und der für die Protonen veränderten Temperaturanisotropie $T_{\perp} / T_{\|}=3$. Der Verlauf dieser Instabilität bei schräger Ausbreitungsrichtung wird durch die Abbildung 2.3a verdeutlicht, in der Höhenlinien der Anwachsrate zu sehen sind. In einem Plasma mit Protonenstrahl ist bei negativen Wellenzahlen $k_{\|}$der in der Abbildung 2.1b gepunktet dargestellte Dispersionszweig von der Instabilität durch die Temperaturanisotropie des Kerns der Verteilung der Protonen betroffen.

Von den Instabilitäten, die durch einen Protonenstrahl ausgelöst werden können, sind sowohl die Alfvén-Moden als auch die magnetosonischen Moden betroffen Montgomery et al. (1975, 1976), Daughton und Gary (1998). Es existieren in dem Parameterbereich, der dem Sonnenwind zugänglich ist, zwei Strahl-Instabilitäten unter den parallel bzw. schräg zum mittleren magnetischen Feld laufenden magnetosonischen Moden. Beide Instabilitäten treten bei negativen Wellenzahlen auf ${ }^{3}$. Unter den Alfvén-Moden können bei der Ausbreitung schräg zum mittleren magnetischen Feld drei verschiedene Instabilitäten

\footnotetext{
${ }^{3}$ In übereinstimmung mit der in den Abbildungen 2.1a und 2.1b aufgegriffenen Konvention, besitzen die magnetosonischen Moden negative Frequenzen. Es muß die generelle Symmetrie $\vec{k} \rightarrow-\vec{k}, \omega \rightarrow-\omega^{*}$ der Dispersionsbeziehung beachtet werden, die es erlaubt, jeweils einen der durch sie verbundenen Dispersionszweige zu unterschlagen.
} 
(a)

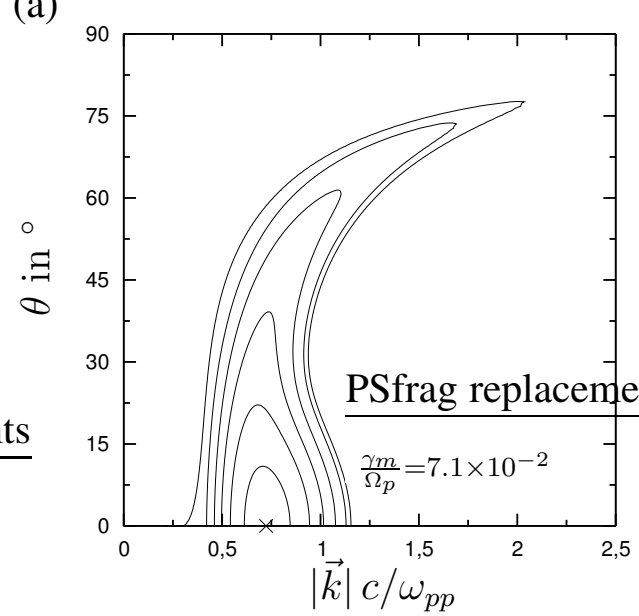

(c)

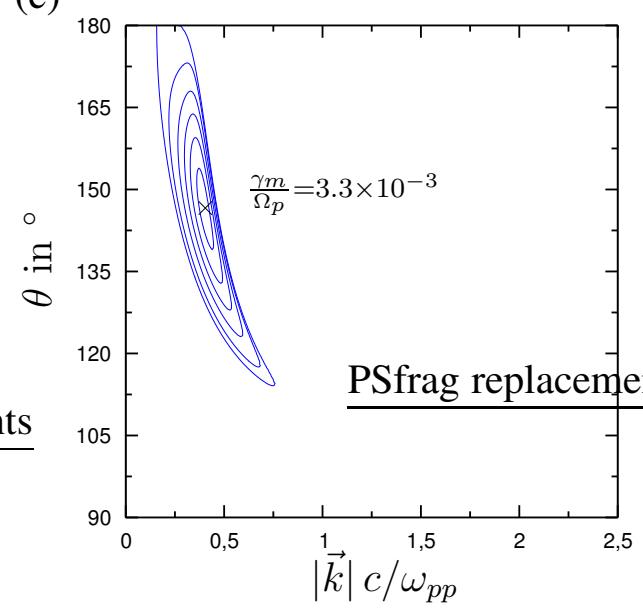

(b)

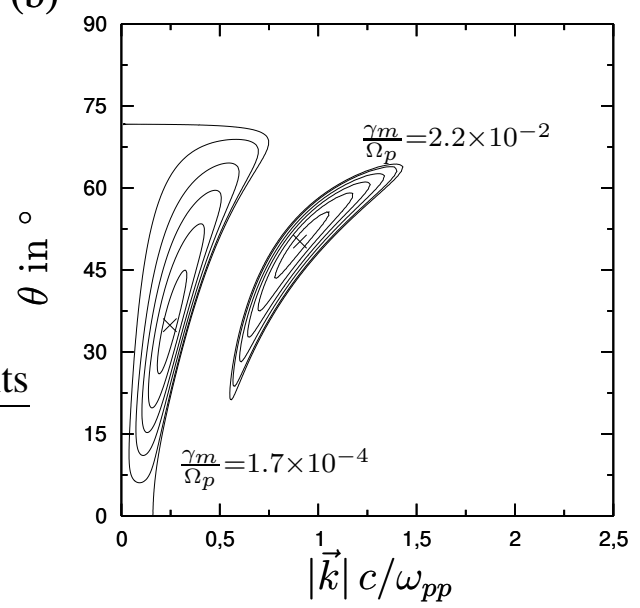

(d)

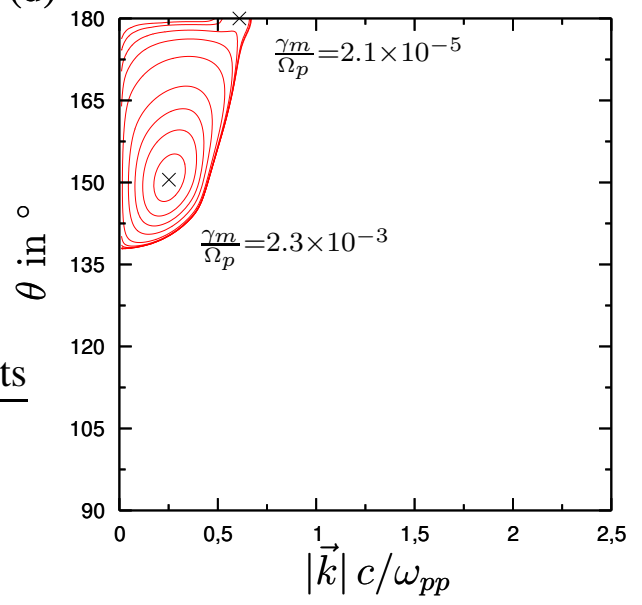

Abbildung 2.3: Höhenlinien der Anwachsraten (a) der Temperaturanisotropie-Instabilität, (b) der Alfvén I und II Instabilitäten, (c) der Alfvén III Instabilität sowie (d) der magnetosonischen Instabilitäten bei paralleler und schräger Ausbreitungsrichtung. Es liegen (a) und (b)-(d) die Parameter der Tabelle 2.1 mit $T_{\perp, p} / T_{\|, p}=3$ bzw. der Tabelle 2.2 zugrunde (vgl. Abbildung 2.2). Die Höhenlinien entsprechen für jede Instabilität den Anteilen 0.9, 0.7, 0.5, 0.3, 0.1 und 0 der maximalen Anwachsrate. Im Fall der magnetosonischen Instabilitäten sind zusätzlich die Höhenlinien zu den Anteilen 0.03, 0.01 und 0.003 der maximalen Anwachsrate zu sehen.

auftreten: die Alfvén I und II Instabilitäten bei positiven sowie die Alfvén III Instabilität bei negativen Wellenzahlen $k_{\|}$. Die Bezeichnung der Alfvén I-III Instabilitäten, die ich im verbleibenden Teil der Arbeit beibehalten werde, geht auf Daughton und Gary (1998) zurück. Die Abbildungen 2.2b und 2.3b-2.3d verdeutlichen den Verlauf der Anwachsraten der genannten Strahl-Instabilitäten und ihre relativen Größenordnungen. Die angegebenen Ausbreitungswinkel $\theta$ der Moden maximaler Anwachsrate beziehen sich auf die durch den Protonenstrahl bestimmte Richtung. Die Dispersionsbeziehung hängt nicht von der Orientierung des mittleren magnetischen Feldes ab. Es liegen den Abbildungen die in der Tabelle 2.2 angegebenen Parameter zugrunde. In diesem Plasma treten alle Strahl-Instabilitäten gemeinsam auf. Die Ausbreitungsrichtung einer Wellenmode ist durch die Phasengeschwindigkeit $\left(\omega /|\vec{k}|^{2}\right) \vec{k}$ vorgegeben. Sie ist invariant unter der be- 
(a)

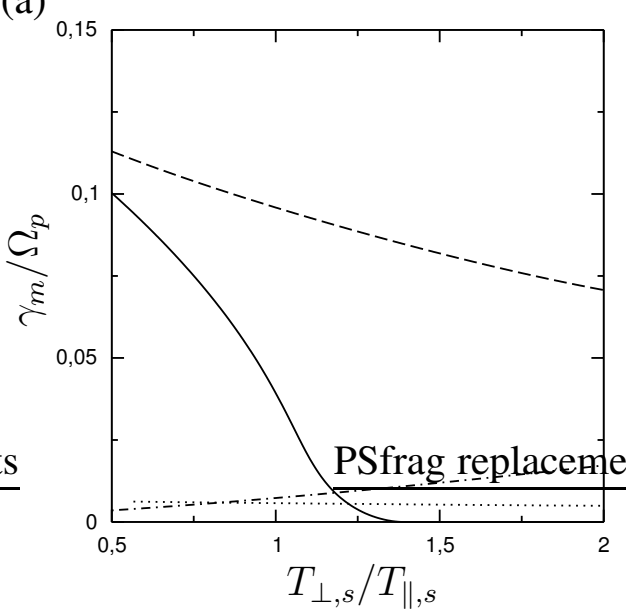

(c)

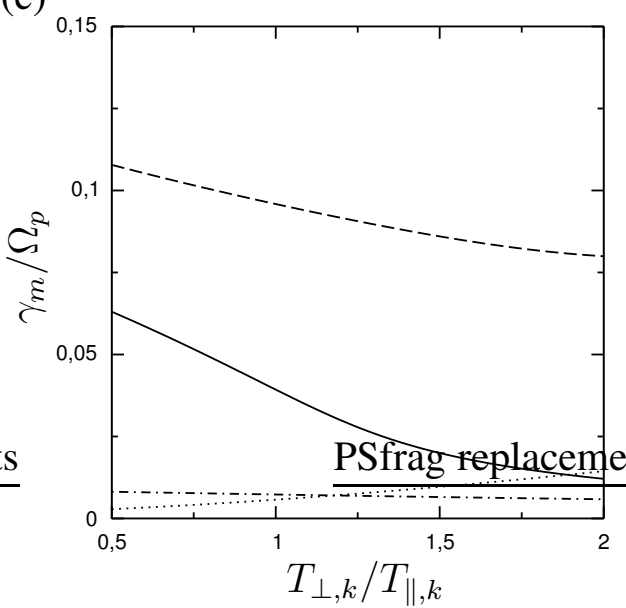

(e)

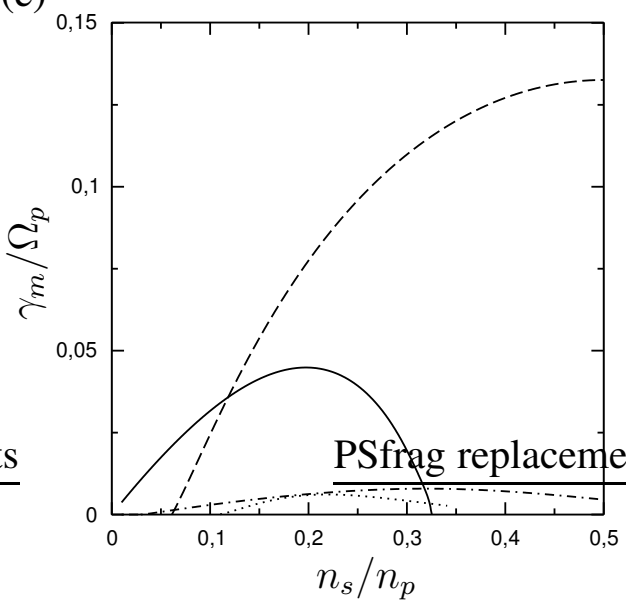

(b)

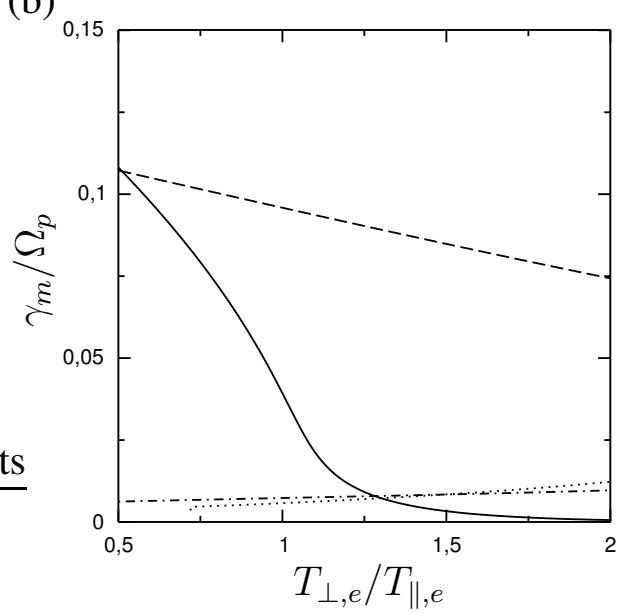

(d)

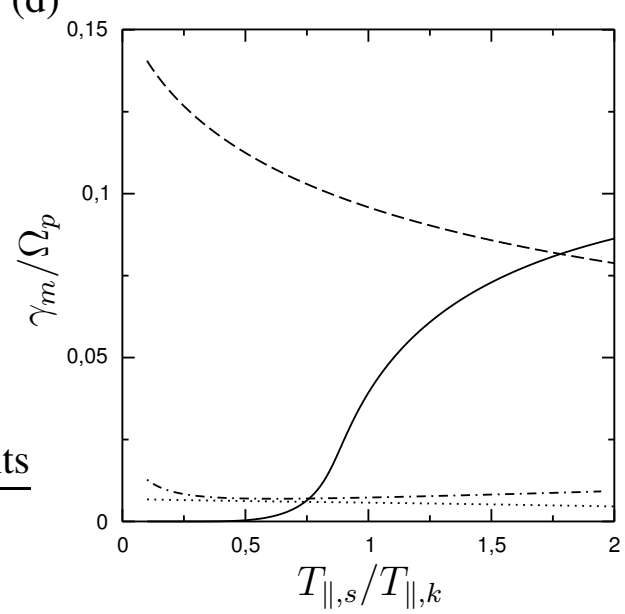

(f)

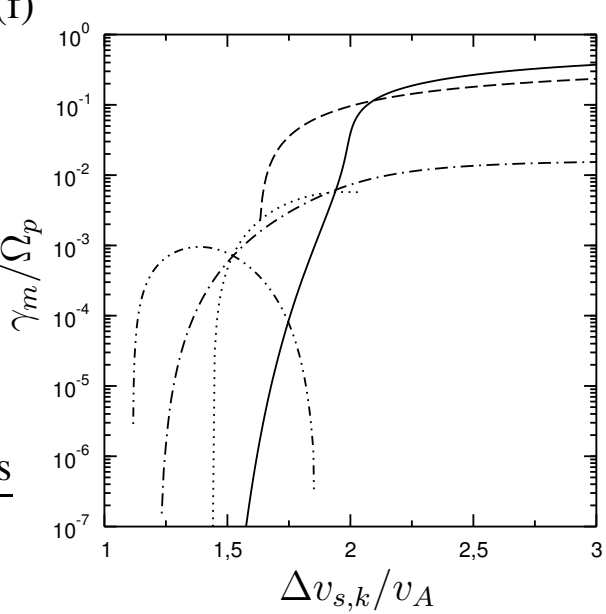

Abbildung 2.4: Abhängigkeit der maximalen Anwachsrate der Strahl-Instabilitäten von (a) $T_{\perp, s} / T_{\|, s}$, (b) $T_{\perp, e} / T_{\|, e}$, (c) $T_{\perp, k} / T_{\|, k}$, (d) $T_{\|, s} / T_{\|, k}$ und (e) $n_{s} / n_{e}$ sowie (f) von der Strahl-Geschwindigkeit ( parallel magnetosonisch (-), Alfvén I (---), schräg magnetosonisch ( (--), Alfvén III ( (.....), Alfvén II (-.-)). Es liegen (a)-(f) bis auf $\Delta v_{k, s}=2.0 v_{A}$ die Parameter der Tabelle 2.2 zugrunde. Die höhere Strahlgeschwindigkeit wurde gewählt, um den direkten Vergleich mit den Ergebnissen von Daughton und Gary (1998) zu ermöglichen. Die Alfvén II Instabilität existiert bei dieser Geschwindigkeit des Protonenstrahls nicht mehr, wie in der Abbildung (f) zu erkennen ist. 
(a)

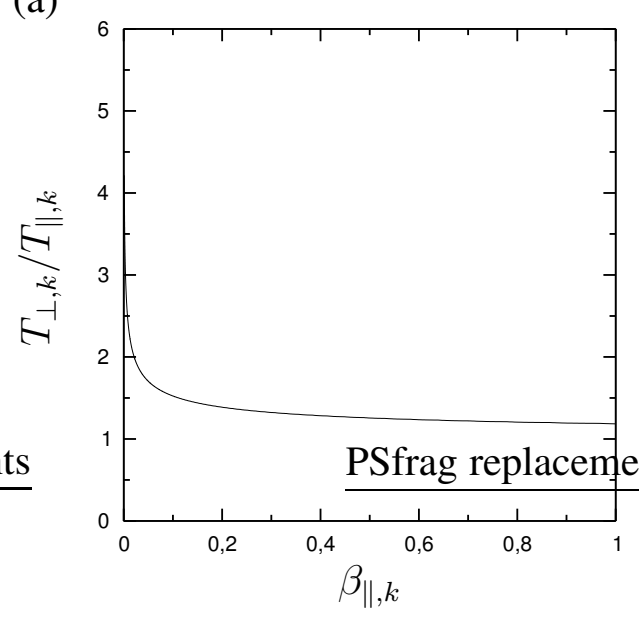

(b)

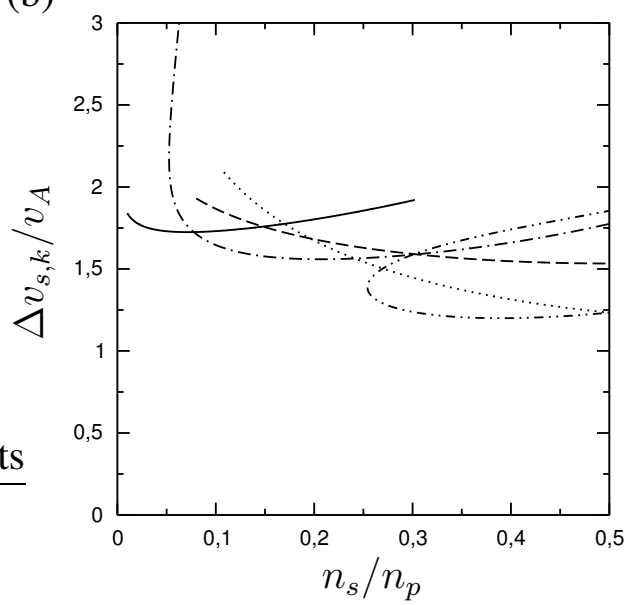

Abbildung 2.5: (a) Kurve konstanter Anwachsrate $\gamma_{m} / \Omega_{p}=10^{-3}$ der Anisotropie-Instabilität in der gezeigten Parameterebene für die Plasmaparameter der Tabelle 2.1. (b) Kurven konstanter Anwachsrate $\gamma_{m} / \Omega_{p}=10^{-3}$ der Strahl-Instabilitäten in der gezeigten Parameterebene für die Plasmaparameter der Tabelle 2.2 ( parallel magnetosonisch (-), Alfvén I (---), schräg magnetosonisch (---), Alfvén III (......), Alfvén II (-.--)).

reits an vorausgegangener Stelle im Text und in der Fußnote 3 erwähnten Symmetrie der Dispersionsbeziehung. Es läßt sich festhalten, daß auf das Ruhesystem des Plasmas bezogen die instabilen magnetosonischen Moden sowie die Moden der Alfvén I und II Instabilitäten auswärts, die Alfvén III hingegen einwärts laufen. Dabei wird die durch den Protonenstrahl vorgegebene Richtung als auswärts gerichtet bezeichnet, was der Situation im Sonnenwind geschuldet ist.

Eine besondere Bedeutung kommt der Abhängigkeit der Instabilitäten von den Plasmaparametern und insbesondere den Stabilitätsgrenzen zu, an denen die linearen Anwachsraten bestimmte konstante Werte überschreiten. Dem Anwachsen der Fluktuationen eines Plasmas aufgrund einer linearen Instabilität ist dadurch ein zeitliches Ende gesetzt, daß die kinetische und die elektromagnetische Energie zusammen nicht-linear erhalten sind. Eine Instabilität muß relaxieren und ihre Anwachsrate muß sich unter eine Stabilitätsgrenze bewegen, die idealerweise durch $\gamma_{m} / \Omega_{p}=0$ bestimmt ist. Diese Stabilitätsgrenze gilt für die lineare Anwachsrate, wenn sie mit der tatsächlichen Anwachsrate übereinstimmt. Korrekturen aufgrund der Nichtlinearität der Fluktuationen (s. Gleichung 2.8) müssen vernachlässigbar sein. In einem inhomogenen Plasma kann der Grenzwert der Anwachsrate einen positiven Wert besitzen. Für eine typische Ausbreitungsgeschwindigkeit $V$ von Wellen im Plasma und eine typische Skala $L$ der Inhomogenität des Plasmas ist dieser Grenzwert von der Größenordnung $\gamma_{m} \approx V / L$. Im schnellen Sonnenwind stimmt die Ausbreitungsgeschwindigkeit nahezu mit der Strömungsgeschwindigkeit überein, $V \approx 700 \mathrm{~km} / \mathrm{s}$, und die Skala der Inhomogenität darf durch $L \approx 0.1 \mathrm{AE}$ abgeschätzt werden, d.h. $\gamma_{m} \approx 5 \times 10^{-5} \mathrm{~s}^{-1}$. Es ist unbekannt, ob die linearen Anwachsraten von Instabilitäten im schnellen Sonnenwind marginal stabil und somit durch diesen Wert beschränkt sind. Das allgemein als gültig angenommene Prinzip der marginalen Stabilität und die Bedeutung der Nichtlinearität müssen überprüft werden. Die Abbildungen 2.4a- 
2.4f zeigen die Abhängigkeit der maximalen Anwachsraten der Strahl-Instabilitäten von den Plasmaparametern, wie sie auch von Daughton und Gary (1998) betrachtet wurden. Die Alfvén I Instabilität und die Instabiltät der sich parallel zum mittleren magnetischen Feld ausbreitenden magnetosonischen Moden sind nach ihrer Anwachsrate beurteilt dominant. Mit wachsender Relativgeschwindigkeit des Protonenstrahls treten sie allerdings nicht zuerst auf. In der Abbildung 2.5b sind ihre willkürlich durch $\gamma_{m} / \Omega_{p}=10^{-3}$ definierten Stabilitätsgrenzen zu sehen, wie sie zwischen der Dichte des Protonenstrahls und seiner Geschwindigkeit relativ zum Kern der Verteilung bestehen. Für kleine Dichten des Strahls gibt die magnetosonische Instabilität bei paralleler Ausbreitungsrichtung die obere Schranke für seine Relativgeschwindigkeit vor. Es folgen mit steigender Dichte die magnetosonische Instabilität bei schräger Ausbreitungsrichtung, die Alfvén III und Alfvén II Instabilität. Diese Instabilitäten treten bereits bei kleineren Relativgeschwindigkeiten des Strahls auf als die Alfvén I Instabilität (vgl. Abbildung 2.4f).

\subsection{Quasi-lineare Diffusion}

Im vorausgegangen Abschnitt über Plasmawellen wurde ein Hintergrund von stationären und homogenen mittleren Feldern angenommen, der von den Plasmawellen unbeeinflußt bleibt. Die Bewegungsgleichung der Geschwindigkeitsverteilungen 2.7 enthält allerdings auf ihrer rechten Seite einen Term, der eine Wechselwirkung zwischen den Plasmawellen und den mittleren Feldern ausdrückt. In der quasi-linearen Näherung der Welle-TeilchenWechselwirkung wird die Veränderung der Geschwindigkeitsverteilungen durch die Plasmawellen über diesen Term berücksichtigt, während die Wellen selbst weiterhin unter der Annahme schwacher Turbulenz linearisiert betrachtet werden. Diese Näherung erlaubt es bereits, die zuvor angesprochene Relaxation von Mikroinstabilitäten zu beschreiben (s. z.B. Davidson 1972). Durch den Wechselwirkungsterm unterliegen die Geschwindigkeitsverteilungen einer Diffusion, der sogenannten quasi-linearen Diffusion.

Die linearisierte Gleichung der Fluktuationen im Plasma 2.8 läßt sich entlang der Teilchentrajektorien in den mittleren elektromagnetischen Feldern integrieren. Es bezeichne $\vec{x}_{t}, \vec{v}_{t}$ eine solche Trajektorie mit der Anfangsbedingung $\vec{x}_{0}=\vec{x}, \vec{v}_{0}=\vec{v}$, dann gilt

$$
\begin{aligned}
\delta f_{s}(\vec{x}, \vec{v}, t)= & \delta f_{s}\left(\vec{x}_{-\Delta t}, \vec{v}_{-\Delta t}, t-\Delta t\right) \\
& -\int_{0}^{\Delta t} d \tau \delta \vec{a}_{s}\left(\vec{x}_{-\tau}, \vec{v}_{-\tau}, t-\tau\right) \cdot \frac{\partial\left\langle f_{s}\right\rangle}{\partial \vec{v}}\left(\vec{x}_{-\tau}, \vec{v}_{-\tau}, t-\tau\right) .
\end{aligned}
$$

Der Welle-Teilchen-Wechselwirkungsterm schreibt sich damit

$$
\begin{aligned}
& -\left\langle\delta \vec{a}_{s} \cdot \frac{\partial \delta f_{s}}{\partial \vec{v}}\right\rangle=-\frac{\partial}{\partial \vec{v}} \cdot\left\langle\delta \vec{a}_{s}(\vec{x}, \vec{v}, t) \delta f_{s}\left(\vec{x}_{-\Delta t}, \vec{v}_{-\Delta t}, t-\Delta t\right)\right\rangle \\
& +\frac{\partial}{\partial \vec{v}} \cdot \int_{0}^{\Delta t} d \tau\left\langle\delta \vec{a}_{s}(\vec{x}, \vec{v}, t) \delta \vec{a}_{s}\left(\vec{x}_{-\tau}, \vec{v}_{-\tau}, t-\tau\right)\right\rangle \cdot \frac{\partial\left\langle f_{s}\right\rangle}{\partial \vec{v}}\left(\vec{x}_{-\tau}, \vec{v}_{-\tau}, t-\tau\right) .
\end{aligned}
$$

Eine wichtige Annahme der quasi-linearen Näherung ist die Dekorrelation der elektromagnetischen Fluktuationen mit großem räumlichen und zeitlichen Abstand, d.h. entlang 
einer Trajektorie soll

$$
\left\langle\delta \vec{a}_{s}(\vec{x}, \vec{v}, t) \delta \vec{a}_{s}\left(\vec{x}_{-\tau}, \vec{v}_{-\tau}, t-\tau\right)\right\rangle \rightarrow 0, \quad \tau \rightarrow \infty
$$

gelten. Messungen der elektromagnetischen Fluktuationen werden mit wachsendem Abstand statistisch unabhängig voneinander. Sind nun die Inhomogenität und Nicht-Stationarität der Geschwindigkeitsverteilungen so gering, daß sie entlang der Trajektorie innerhalb einer typischen Dekorrelationsskala der elektromagnetischen Fluktuationen nicht variieren

$$
\frac{\partial\left\langle f_{s}\right\rangle}{\partial \vec{v}}\left(\vec{x}_{-\tau}, \vec{v}_{-\tau}, t-\tau\right) \approx \frac{\partial\left\langle f_{s}\right\rangle}{\partial \vec{v}}\left(\vec{x}, \vec{v}_{-\tau}, t\right), \quad \tau \lesssim \tau_{c}
$$

dann hängt die Welle-Teilchen-Wechselwirkung nur noch von der momentanen Geschwindigkeitsverteilung ab und nicht von ihrer Geschichte im Integrationsintervall. Wegen der Dekorrelationseigenschaft 2.32 kann, ohne daß dies einen großen Fehler verursacht, die Integration innerhalb der Gleichung 2.31 bis unendlich ausgedehnt werden. Wenn im gleichen Zug die verbleibende Korrelation zwischen den zeitlich versetzten Fluktuationen der Mikrodichten und den elektromagnetischen Feldern vernachlässigt wird,

$$
\left\langle\delta \vec{a}_{s}(\vec{x}, \vec{v}, t) \delta f_{s}\left(\vec{x}_{-\Delta t}, \vec{v}_{-\Delta t}, t-\Delta t\right)\right\rangle \approx 0,
$$

ergibt sich die Diffusionsgleichung

$$
-\left\langle\delta \vec{a}_{s} \cdot \frac{\partial \delta f_{s}}{\partial \vec{v}}\right\rangle \approx \frac{\partial}{\partial \vec{v}} \cdot\left(\mathbf{D}_{s}(\vec{x}, \vec{v}, t) \cdot \frac{\partial\left\langle f_{s}\right\rangle}{\partial \vec{v}}(\vec{x}, \vec{v}, t)\right)
$$

mit der Diffusionsmatrix

$$
\mathbf{D}_{s}=\int_{0}^{\infty} d \tau\left\langle\delta \vec{a}_{s}(\vec{x}, \vec{v}, t) \delta \vec{a}_{s}\left(\vec{x}_{-\tau}, \vec{v}_{-\tau}, t-\tau\right)\right\rangle \cdot \mathbf{R}\left(-\Omega_{s} \tau\right),
$$

wobei mit $\mathbf{R}(\phi)$ die Rotationsmatrix zum Winkel $\phi$ um das mittlere magnetische Feld gemeint ist. Dafür müssen die Geschwindikeitsverteilungen symmetrisch um das magnetische Feld sein. Diese Annahme wurde bereits für die Berechnung der Dispersion von Plasmawellen gemacht. Unter der Annahme nur schwach inhomogener und nichtstationärer elektromagnetischer Fluktuationen, z.B.

$$
\left\langle\delta \vec{B}(\vec{x}, t) \delta \vec{E}\left(\vec{x}+\vec{x}^{\prime}, t+t^{\prime}\right)\right\rangle \approx\left\langle\delta \vec{B}\left(\vec{x}-\vec{x}^{\prime}, t-t^{\prime}\right) \delta \vec{E}(\vec{x}, t)\right\rangle,
$$

kann der allgemeine Ausdruck 2.36 der Diffusionsmatrix ausgewertet werden. Durch die Fourier-Transformation in den Abständen lassen sich alle Korrelationen auf das Spektrum der Fluktuationen des elektrischen Feldes

$$
\mathbf{P}_{E}(\vec{x}, t ; \omega, \vec{k})=\int \frac{d t^{\prime} d^{3} x^{\prime}}{(2 \pi)^{2}}\left\langle\delta \vec{E}(\vec{x}, t) \delta \vec{E}\left(\vec{x}+\vec{x}^{\prime}, t+t^{\prime}\right)\right\rangle e^{i\left(\omega t^{\prime}-\vec{k} \cdot \vec{x}^{\prime}\right)}
$$

zurückführen. Genauso wie die Geschwindigkeitsverteilungen der Teilchenspezies sollen auch die Korrelationen des elektrischen Feldes symmetrisch um das mittlere magnetische Feld sein, d.h. es gilt $\mathbf{P}_{E}(\vec{x}, t ; \omega, \mathbf{R} \cdot \vec{k}) \approx \mathbf{R} \cdot \mathbf{P}_{E}(\vec{x}, t ; \omega, \vec{k}) \cdot \mathbf{R}^{T}$ für alle Rotationen $\mathbf{R}$ 
um das mittlere magnetische Feld. Diese Eigenschaft der Fluktuationen hat die wichtige Folge, daß die Symmetrie der Geschwindigkeitsverteilungen im Verlauf der Diffusion erhalten bleibt und sich die Diffusionsgleichung 2.35 in der Form

$$
\begin{aligned}
-\left\langle\delta \vec{a}_{s} \cdot \frac{\partial \delta f_{s}}{\partial \vec{v}}\right\rangle= & \frac{\partial}{\partial v_{\|}}\left(D_{\|\| \|}^{s} \frac{\partial\left\langle f_{s}\right\rangle}{\partial v_{\|}}+D_{\| \perp}^{s} \frac{\partial\left\langle f_{s}\right\rangle}{\partial v_{\perp}}\right) \\
& +\frac{1}{v_{\perp}} \frac{\partial}{\partial v_{\perp}} v_{\perp}\left(D_{\| \perp}^{s} \frac{\partial\left\langle f_{s}\right\rangle}{\partial v_{\|}}+D_{\perp \perp}^{s} \frac{\partial\left\langle f_{s}\right\rangle}{\partial v_{\perp}}\right)
\end{aligned}
$$

schreiben läßt, in der nunmehr allein die $v_{\|}$- und $v_{\perp}$-Abhängigkeiten eine Rolle spielen. Die Diffusionskoeffizienten sind (s. z.B. Kennel und Engelmann 1966)

$$
\begin{aligned}
D_{\|\|}^{s} & =\left(\frac{e_{s}}{m_{s}}\right)^{2} \sum_{n=-\infty}^{\infty} \int \frac{d^{3} k d \omega}{(2 \pi)^{2}} R\left(\omega-k_{\|} v_{\|}-n \Omega_{s}\right) \vec{\alpha}_{n \|}^{*} \cdot \mathbf{P}_{E} \cdot \vec{\alpha}_{n \|}, \\
D_{\| \perp}^{s} & =\left(\frac{e_{s}}{m_{s}}\right)^{2} \sum_{n=-\infty}^{\infty} \int \frac{d^{3} k d \omega}{(2 \pi)^{2}} R\left(\omega-k_{\|} v_{\|}-n \Omega_{s}\right) \vec{\alpha}_{n \|}^{*} \cdot \mathbf{P}_{E} \cdot \vec{\alpha}_{n \perp}, \\
D_{\perp \|}^{s} & =\left(\frac{e_{s}}{m_{s}}\right)^{2} \sum_{n=-\infty}^{\infty} \int \frac{d^{3} k d \omega}{(2 \pi)^{2}} R\left(\omega-k_{\|} v_{\|}-n \Omega_{s}\right) \vec{\alpha}_{n \perp}^{*} \cdot \mathbf{P}_{E} \cdot \vec{\alpha}_{n \|}
\end{aligned}
$$

und

$$
D_{\perp \perp}^{s}=\left(\frac{e_{s}}{m_{s}}\right)^{2} \sum_{n=-\infty}^{\infty} \int \frac{d^{3} k d \omega}{(2 \pi)^{2}} R\left(\omega-k_{\|} v_{\|}-n \Omega_{s}\right) \vec{\alpha}_{n \perp}^{*} \cdot \mathbf{P}_{E} \cdot \vec{\alpha}_{n \perp}
$$

wobei die Abkürzungen

$$
\begin{aligned}
\vec{\alpha}_{n \|} & =\left(1-\frac{n \Omega_{s}}{\omega}\right) J_{n} \vec{b}+\frac{k_{\|} v_{\perp}}{\sqrt{2} \omega}\left(J_{n+1} e^{i \psi} \vec{e}_{+}+J_{n-1} e^{-i \psi} \vec{e}_{-}\right) \\
\vec{\alpha}_{n \perp} & =\frac{n \Omega_{s}}{\omega} \frac{v_{\|}}{v_{\perp}} J_{n} \vec{b}+\frac{1}{\sqrt{2}}\left(1-\frac{k_{\|} v_{\|}}{\omega}\right)\left(J_{n+1} e^{i \psi} \vec{e}_{+}+J_{n-1} e^{-i \psi} \vec{e}_{-}\right) \\
\vec{e}_{ \pm} & =\left(\vec{e}_{1} \pm i \vec{e}_{2}\right) / \sqrt{2}, \quad \vec{k}=k_{\|} \vec{b}+k_{\perp}\left(\cos \psi \vec{e}_{1}+\sin \psi \vec{e}_{2}\right)
\end{aligned}
$$

und die Distribution

$$
R(\omega)=\frac{i}{\omega+i 0^{+}}=\pi \delta(\omega)+i P \frac{1}{\omega}
$$

auftauchen. Die Voraussetzungen für die quasi-linearen Diffusion 2.35 bzw. 2.39 und der Diffusionskoeffizienten 2.40-2.43lauten zusammengefaßt: Dekorrelation der Fluktuationen, eine gegenüber den Skalen der mittleren Felder kleine Dekorrelationsskala (Skalentrennung), die Symmetrie der Fluktuationen um das magnetische Feld sowie ihre Homogenität und Stationarität.

Für die Wechselwirkung mit den Teilchen werden im Sonnenwind sich parallel zum mittleren magnetischen Feld ausbreitende Wellenmoden verantwortlich gemacht. Diese sollen ihren Ursprung auf der Sonne besitzen und entlang des magnetischen Feldes auswärts laufen. Demnach besäße das Spektrum $\mathbf{P}_{E}$ sein Hauptgewicht bei Wellenvektoren mit $k_{\perp}=0$. Die Richtigkeit dieser Annahme ist unklar. So tritt z.B. die maximale Anwachsrate der im Abschnitt 2.2 dieses Kapitels beschriebenen Strahl-Instabilitäten bei schräger 
Ausbreitungsrichtung auf. In dieser Arbeit betrachte ich nur sich parallel zum mittleren magnetischen ausbreitende Wellenmoden. Dadurch vereinfachen sich die Diffusionskoeffizienten und nehmen die Form

$$
\begin{aligned}
D_{\|\|}^{s} & =\pi\left(\frac{e_{s}}{m_{s}}\right)^{2} \int \frac{d^{3} k d \omega}{(2 \pi)^{2}} \delta\left(\omega-k_{\|} v_{\|}\right) P_{E \|} \\
& +\pi\left(\frac{e_{s}}{m_{s}}\right)^{2} \int \frac{d^{3} k d \omega}{(2 \pi)^{2}} \delta\left(\omega-k_{\|} v_{\|}-\Omega_{s}\right) \frac{1}{2}\left(\frac{k_{\|} v_{\perp}}{\omega}\right)^{2} P_{E \perp}, \\
D_{\| \perp}^{s} & =D_{\perp \|}^{s}= \\
= & \pi\left(\frac{e_{s}}{m_{s}}\right)^{2} \int \frac{d^{3} k d \omega}{(2 \pi)^{2}} \delta\left(\omega-k_{\|} v_{\|}-\Omega_{s}\right) \frac{k_{\|} v_{\perp}}{2 \omega}\left(1-\frac{k_{\|} v_{\|}}{\omega}\right) P_{E \perp}
\end{aligned}
$$

und

$$
D_{\perp \perp}^{s}=\pi\left(\frac{e_{s}}{m_{s}}\right)^{2} \int \frac{d^{3} k d \omega}{(2 \pi)^{2}} \delta\left(\omega-k_{\|} v_{\|}-\Omega_{s}\right) \frac{1}{2}\left(1-\frac{k_{\|} v_{\|}}{\omega}\right)^{2} P_{E \perp}
$$

an. Es tauchen die Spektren

$$
P_{E \|}=\vec{b} \cdot \mathbf{P}_{E} \cdot \vec{b}, \quad P_{E \perp}=2 \vec{e}_{-}^{*} \cdot \mathbf{P}_{E} \cdot \vec{e}_{-}
$$

für elektrostatische bzw. Zyklotronwellen auf, deren Eigenschaften im Sonnenwind allerdings unbekannt sind. Die Dispersionsbeziehung bietet den Ansatzpunkt

$$
P_{E \perp}(\omega, \vec{k}) \approx(2 \pi)^{3 / 2} \sum_{M} \delta\left(\omega-\omega_{k_{\|}}^{M}\right) \delta\left(\vec{k}_{\perp}\right) P_{E \perp}^{M}\left(k_{\|}\right)
$$

wobei prinzipiell alle Dispersionszweige $M$ mit zu $\vec{e}_{-}$parallel gerichteter Polarisation eingehen. Die Wechselwirkung mit elektrostatischen Moden soll vernachlässigt werden. Der unter dem Einfluß des Spektrums 2.52 ablaufende Diffusionsprozeß läßt sich auf einfache Weise charakterisieren. Teilchen mit einer Geschwindigkeit $v_{\|}$wechselwirken mit einer endlichen Anzahl von Moden, deren Wellenzahlen die Resonanzbedingung $\omega_{k_{\|}}-v_{\|} k_{\|}-\Omega_{s}=0$ erfüllen müssen. Falls bei einer Geschwindigkeit $v_{\|}$eine einzige resonante Mode mit der resonanten Wellenzahl $k_{\|}$existiert, dann verläuft die Diffusion bei dieser Geschwindigkeit für jedes $v_{\perp}$ entlang der Richtung

$$
\vartheta_{\|}=v_{\perp} k_{\|} / \omega_{k_{\|}}, \quad \vartheta_{\perp}=1-v_{\|} k_{\|} / \omega_{k_{\|}} .
$$

Sie ist somit auf die Kurven eingeschränkt, zu denen diese Richtungen tangential sind. Die Höhenlinien der Funktion

$$
P\left(v_{\|}, v_{\perp}\right)=v_{\|}^{2}+v_{\perp}^{2}-2 \int^{v_{\|}} d v_{\|}^{\prime} \frac{\omega_{k_{\|}}}{k_{\|}}\left(v_{\|}^{\prime}\right)
$$

stimmen mit den Kurven überein, wobei die Phasengeschwindigkeit $\omega_{k_{\|}} / k_{\|}$der Wellenmoden über die Resonanzbedingung eine Funktion der Geschwindigkeit $v_{\|}$ist. Die Diffusion führt mit fortschreitender Zeit dazu, daß die Geschwindigkeitsverteilung konstant auf 
(a)

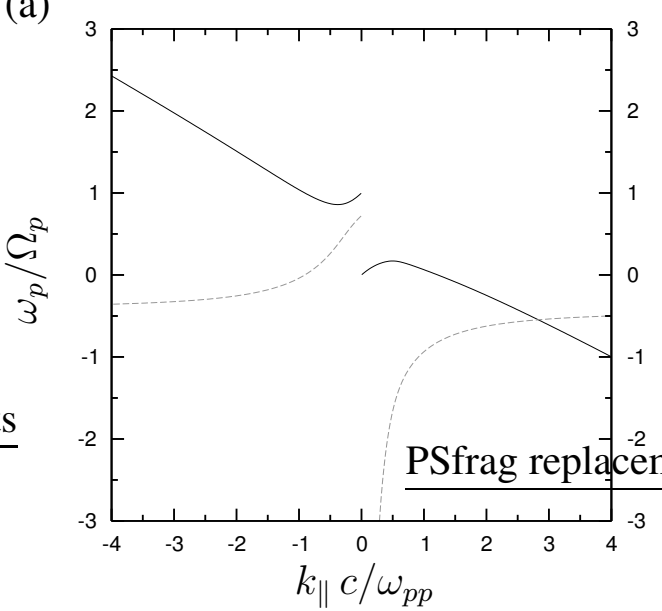

(b)

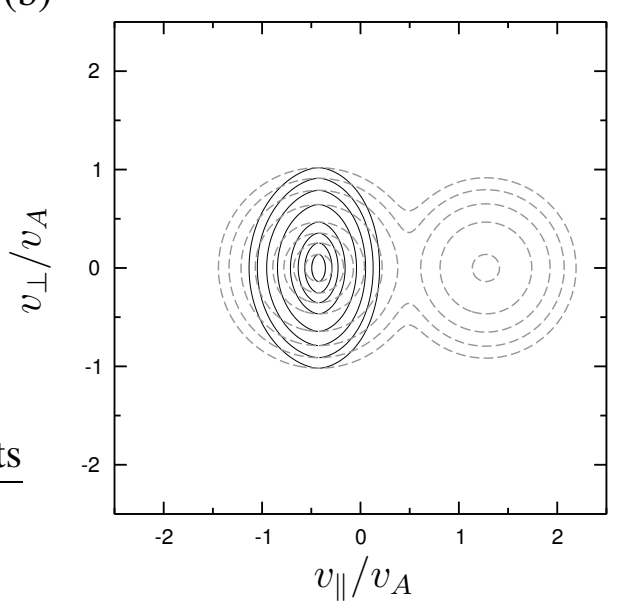

Abbildung 2.6: (a) Dispersionszweige der mit dem Kern der Geschwindigkeitsverteilung der Protonen resonanten Moden. Die gestrichelte Gerade stellt den Verlauf der Resonanzgeschwindigkeit $\left(v_{\text {res }}=\left(\omega-\Omega_{p}\right) / k_{\|}\right)$dar. Es wurden die Plasmaparameter der Tabelle 2.2 zugrunde gelegt. (b) Höhenlinien der Geschwindigkeitsverteilung der Protonen, die den Bruchteilen 0.9, 0.7, 0.5, 0.3, 0.1, 0.03, 0.01 und 0.003 des Maximums der Verteilung entsprechen (gestrichelte Kurven). Es sind ebenfalls die Diffusionsplateaus dargestellt, die die gleichen $v_{\perp}$-Achsenabschnitte im Zentrum des Kerns besitzen wie die gezeigten Höhenlinien.

den Diffusionskurven wird. Existieren mehrere resonante Moden, dann ist die Diffusionsrichtung eine Superposition der jeweiligen Richtungen 2.53, die von den Amplituden der betreffenden Moden abhängt. In diesem Fall kann eine Geschwindigkeitsverteilung in der Regel nur stationär sein, wenn sie bei den resonanten Geschwindigkeiten entlang jeder Richtung konstant ist. Die Abbildung 2.6b zeigt Diffusionskurven zu der Geschwindigkeitsverteilung der Protonen, die den in der Tabelle 2.2 genannten Parametern entspricht. In der Abbildung 2.6a sind die Dispersionszweige der resonanten Moden, zu denen diese Diffusionskurven gehören, zusammen mit den Resonanzgeschwindigkeiten dargestellt (vgl. Abbildung 2.1b). Die Diffusionskurven verlaufen innerhalb der Kerns der Verteilung. Sie stimmen allerdings nicht mit den überlagerten Höhenlinien der Verteilung überein, die im Zentrum des Kerns dieselben $v_{\perp}$-Achsenabschnitte besitzen. Es wird sich im Kapitel 7 zeigen, daß anders als in diesem Beispiel im schnellen Sonnenwind eine weitgehende und systematische Übereinstimmung festgestellt werden kann. Es lassen sich Diffusionsplateaus im Kern der Geschwindigkeitsverteilungen der Protonen beobachten. 


\section{Meßdaten}

\subsection{Helios}

Die Helios-Mission bestand aus zwei Raumsonden. Helios 1 startete am 10. Dezember 1974 und Helios 2 am 15. Januar 1976. Beide wurden auf exzentrische elliptische Umlaufbahnen um die Sonne gebracht, die sich innerhalb der Erdumlaufbahn befanden. Helios ist bislang die einzige Mission, mit der Eigenschaften und Prozesse des interplanetaren Mediums der innersten Heliosphäre in situ gemessen werden konnten. Die Umlaufbahnen der Helios-Sonden hatten ihren sonnennächsten Punkt bei 0.3 AE für Helios 1 und 0.29 AE für Helios 2, d.h. innerhalb der Merkur-Umlaufbahn. Die Inklination der Bahnen gegenüber der Ekliptik war kleiner als $1^{\circ}$. Beide Helios-Sonden waren im wesentlichen baugleich. Auf beiden Sonden arbeiteten zehn Experimente mit verschiedenen wissenschaftlichen Zielsetzungen. Sie lassen sich in drei Gruppen einteilen:

1. Experimente über das interplanetare Plasma (Sonnenwind),

2. Experimente über die kosmische Strahlung und

3. Experimente über Mikrometeoriten.

Dieser Arbeit liegen sowohl die Daten des Plasma- als auch des Magnetometer-Experimentes zugrunde (Rosenbauer et al. 1981, Musmann et al. 1975). Das Plasmaexperiment lieferte in Zeitintervallen von 40.5 s einen Meßdatensatz. Die Ergebnisse der Magnetfeldmessungen, deren zeitliche Auflösung 0.125 s beträgt, wurden zu 32 s Mittelwerten innerhalb eines Meßzyklus des Plasmaexperimentes zusammenfaßt ${ }^{1}$. Die Meßdaten stehen vom 12. Dezember 1974 bis zum 4. September 1985 für Helios 1 und vom 20. Februar 1976 bis zum 8. März 1980 für Helios 2 zur Verfügung. Zu Beginn der Messungen auf Helios 1 und 2 befand sich die Sonne unmittelbar vor einem Aktivitätsminimum. Wie die Abbildung 3.1 zeigt, in welcher der zeitliche Verlauf der Sonnenfleckenanzahl dargestellt ist, stieg ihre Aktivität in den Folgejahren bis 1980 an und erreichte in diesem Jahr ein Maximum. Der solare Zyklus zeigt sich auch in den Eigenschaften des interplanetaren Mediums. So weisen u.a. der Protonenfluß des Sonnenwindes, die relative Häufigkeit von $\alpha$-Teilchen im Sonnenwind und das interplanetare magnetische Feld eine dem Aktivitätszyklus folgende Variabilität auf (s. z.B. Ogilvie und Hirshberg 1974, Feldman et al. 1978, Smith und Bieber 1991, Aellig et al. 2001). Die Primärphasen beider Helios-Sonden, die

\footnotetext{
${ }^{1}$ Die Instrumente des Plasmaexperimentes benötigten maximal $32 \mathrm{~s}$ zur Erstellung eines Datensatzes. In den verbleibenden acht Sekunden fand keine Messung statt.
} 


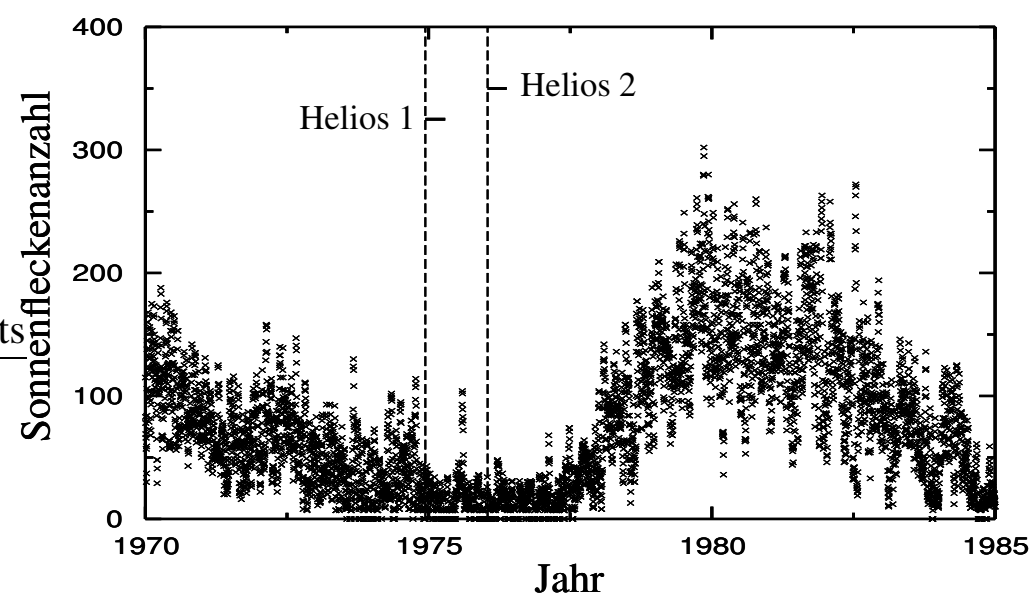

Abbildung 3.1: Tägliche Anzahl der Sonnenflecken zwischen den Jahren 1970 und 1985 (RWC Belgium, World Data Center for the Sunspot Index)

für Helios 1 bis zum 17. April 1975 und für Helios 2 bis zum 17. Mai 1976 dauerte, fallen vollständig in das anfängliche Aktivitätsminimum. Es ist dieser zeitliche Abschnitt, in dem die Daten des Plasmaexperimentes ihre größte Dichte aufweisen und in dem das Gros aller Daten liegt. Die in dieser Arbeit verwendeten Daten stammen ausschließlich aus diesem solaren Minimum.

\subsubsection{Das Plasmaexperiment}

Das Plasmaexperiment bestand aus vier Instrumenten. Die Instrumente 1a, 1b und 3 maßen Ionen mit spezifischen Energien zwischen $0.5 \mathrm{erg} / \mathrm{esu}$ und $50 \mathrm{erg} / \mathrm{esu}(150 \mathrm{~V}$ $7500 \mathrm{~V})$. Elektronen mit Energien zwischen $1.7 \times 10^{-11} \mathrm{erg}$ und $2.7 \times 10^{-9} \mathrm{erg}(10 \mathrm{eV}$ - $1.7 \mathrm{keV}$ ) wurden durch das Instrument 2 erfaßt. Dieses Instrument besaß einen Meßmodus, in dem auch Elektronen mit Energien unterhalb von $10.68 \mathrm{eV}$ gemessen wurden. Solche Daten wurden in dieser Arbeit nicht verwendet. Die Energiebereiche der Instrumente entsprechen Geschwindigkeiten der Protonen zwischen $200 \mathrm{~km} / \mathrm{s}$ und $1700 \mathrm{~km} / \mathrm{s}$ bzw. Geschwindigkeiten der Elektronen zwischen $2000 \mathrm{~km} / \mathrm{s}$ und $25000 \mathrm{~km} / \mathrm{s}$. Im Gegensatz zu den Instrumenten 1a, 2 und 3, die Ionen bzw. Elektronen nach ihrer Einfallsrichtung und Geschwindigkeit unterschieden, maß das Instrument $1 \mathrm{~b}$ den über einen um die Sichtlinie Helios-Sonne gelegenen Raumwinkel integrierten Ladungsstrom der Ionen. Das Instrument 3 wurde so konzipiert, daß es die Ionen nicht nur nach ihren Geschwindigkeiten sondern auch nach ihrer Masse unterscheiden konnte. Es arbeitete wegen seiner geringeren Empfindlichkeit und größeren Leistungsaufnahme nur im Wechsel mit dem Instrument 1a und auch nur in der Nähe des Perihels der beiden Helios-Sonden. Ich habe die Meßdaten dieses Instrumentes deshalb nicht verwendet. Im Folgenden sollen die wichtigsten Eigenschaften der Instrumente 1a, 1b und 2, auf deren Daten diese Arbeit beruht, beschrieben werden. Technische Details sind Gegenstand des Forschungsberichtes von (Rosenbauer et al. 1981). Auf das Magnetometer-Experiment (Musmann et al. 1975) gehe ich nicht ein, da nur die erwähnten 32 s-Mittelwerte des magnetischen Feldes 
verwendet werden und kein direkter Gebrauch von den Daten des Experimentes gemacht wird.

\section{Instrument 1a}

Das Instrument 1a trennte die in definierten Zeitintervallen einfallenden Ionen des Sonnenwindes und zählte sie. Zu diesem Zweck bestand es aus einem elektrostatischen Viertelkugel-Analysator und neun Teilchendetektoren. Einen solchen Analysator passieren nur die Ionen, deren spezifische Energie in einen durch die Steuerungsspannung vorgegebenen Durchlaßbereich fällt und deren Einfallsrichtung in der Schnittfläche des Analysators liegt. Das Instrument war so montiert, daß die Rotationsachse der Helios-Sonden mit der zentralen Achse der Viertelkugel übereinstimmte. Innerhalb einer Rotationsperiode, die eine Sekunde dauerte, wurden alle möglichen Blickrichtungen durchlaufen. Der Azimut der Einfallsrichtung eines Ions relativ zu der nach außen gerichteten Verbindungslinie Sonne-Helios ist durch den zeitlichen Bruchteil einer Umdrehung, in dem es gezählt wurde, definiert. Die Elevation der Geschwindigkeit eines Ions über der Ebene der Bahnbewegung wurde durch die neun Detektoren unterschieden, die geeignet an der Austrittsseite des Analysator angebracht waren. Insgesamt besaß das Instrument 1a 32 Energiekanäle, 16 Azimutkanäle und 9 Elevationkanäle. Es maß im allgemeinen in einer Rotationsperiode alle 144 Winkelkanäle aus. Der Energiekanal wurde nach einer vollständigen Rotation weitergeschaltet, so daß die Aufnahme eines vollständigen Spektrums $32 \mathrm{~s}$ dauerte. Die genauen Lagen der Energie- und Winkelkanäle des Instrumentes sind in dem Forschungsbericht von Rosenbauer et al. (1981) auf der S. 181 tabelliert. Die Winkelkanäle waren in guter Näherung äquidistant verteilt, wobei die Winkelabstände im Azimut und in der Elevation ungefähr $5.6^{\circ}$ bzw. $5.1^{\circ}$ betrugen. Die Abstände der Energiekanäle erhöhten sich beginnend mit ungefähr $8 \times 10^{-2} \mathrm{erg} / \mathrm{esu}$ bei dem niedrigsten Energiekanal schrittweise um den nahezu konstanten Faktor 1.16. Die genaue Winkelausrichtung des Kanalgitters hing vom wechselnden Meßmodus des Instrumentes ab (s. Rosenbauer et al. 1981, S. 68 und S. 77ff.). Der Wert der Verteilungsfunktion der Ionen bei der zentralen Geschwindigkeit eines Kanals $f$ leitet sich aus der gemessene Zählrate $Z$ durch die Beziehung

$$
Z=G \cos (\epsilon) v^{4} f
$$

her. Darin bezeichnen $\epsilon$ und $v$ die zentrale Elevation bzw. den Geschwindigkeitsbetrag des betreffenden Kanals ${ }^{2}$. Die mit den Elevationkanälen variierende Größe $G$ hängt mit der sogenannten Gerätefunktion des Kanals zusammen, die durch eine Eichung des Instrumentes bestimmt wurde (s. Rosenbauer et al. 1981, S. 172ff.). Zur Erklärung der Abhängigkeit von $\cos (\epsilon) v^{4}$ ist zu berücksichtigen, daß die in einem Kanal gemessene Zählrate idealerweise proportional zu der eintreffenden Teilchenzahl ist. Diese leitet sich aus der Stromdichte der Teilchen ab, deren Geschwindigkeit in den Durchlaßbereich des betreffenden Kanals fallen. Die Stromdichte muß dafür über die Eintrittsfläche des Instrumentes und die Dauer der Messung integriert werden. Das Instrument spricht auf Teilchen mit verschiedener Geschwindigkeit auch innerhalb eines Kanals nicht gleich empfindlich an. Deshalb ist die Geschwindigkeitsverteilung zur Berechnung der Teilchenstromdichte

\footnotetext{
${ }^{2}$ Für die Umrechnung der spezifischen Energie eines Ions in dessen Geschwindigkeit muß an dieser Stelle angenommen werden, daß es die Ladung und Masse eines Protons besitzt.
} 
mit der von der Geschwindigkeit abhängigen Gerätefunktion des Kanals zu multipliziert. Eine Taylor-Entwicklung der Geschwindigkeitsverteilung um das Kanalzentrum ergibt damit in nullter Ordnung eine Beziehung der Form 3.1, worin die Proportionalität zu $v^{3}$ aus dem Anwachsen des Volumens, d.h des Durchlaßbereiches der Kanäle mit der Geschwindigkeit folgt. Der Faktor $\cos (\epsilon) v$ stammt vom Produkt der vektoriellen Geschwindigkeit der Teilchen mit der Normalen der Eintrittsfläche. Die Meßdauer und die Eintrittsfläche sind in $G$ enthalten.

Von den Helios-Sonden wurden im allgemeinen nicht die Zählraten aller Kanäle des Instrumentes 1a sondern nur diejenigen eines zusammenhängenden Ausschnittes des Kanalgitters übertragen. Zum Ausgleich sind für jeden Meßzyklus bei jedem Energiekanal die über die Winkelkänale summierten Zählraten verfügbar. Diese summierten Zählraten ermöglichen näherungsweise die Berechnung der über die Winkel integrierten Geschwindigkeitsverteilung der Ionen

$$
F(v)=\int d \alpha d \epsilon v^{2} \cos (\epsilon) f(v, \alpha, \epsilon)
$$

worin $\alpha$ und $\epsilon$ der Azimut bzw. die Elevation der Geschwindigkeit und $v$ ihr Betrag sind. Die für einen konstanten Energiekanal summierte Zählrate $Z$ hängt mit dem Wert $F$ der reduzierten Verteilungsfunktion 3.2 bei der dem Energiekanal entsprechenden Geschwindigkeit $v$ über die Beziehung

$$
Z=G_{0} v^{2} F
$$

zusammen, wobei die Konstante $G_{0}$ sich aus den Gerätefunktionen der Kanäle ergibt (s. Rosenbauer et al. 1981, S. 179f.). Die Abbildung 3.2 zeigt die so bestimmte reduzierte Verteilung der Ionen für einen beispielhaften Datensatz. Es ist ein von den Protonen des Sonnenwindes herrührendes Verteilungsmaximum zu erkennen. Welche Ionenspezies in dem bei höherer spezifischer Energie liegenden Nebenmaximum registriert wurden, läßt sich durch die Messung des Instrumentes 1a nicht bestimmen, da es Ionen nur nach ihrer spezifischen Energie unterschieden hat.

\section{Instrument $1 \mathrm{~b}$}

Das Instrument $1 \mathrm{~b}$ maß durch eine geeignete Kombination von elektrostatischem Analysator und Elektrometer in 32 Energie-Kanälen, deren Lagen mit denen des Instrumentes 1a nahezu übereinstimmten, den über einen um die Sichtlinie Sonne-Helios gelegenen Raumwinkel integrierten Ladungsstrom der Ionen. Dieser ist mit dem Sichtbereich des Instruments 1a vergleichbar. Die vom Instrument in einem Energiekanal aufgefangene Ladung ergibt sich aus den einzelnen Geschwindigkeitsverteilungen der Ionen durch die Integration der Ladungsstromdichte, wobei in die Geschwindigkeitsintegration ebenso wie für das Instrument 1a eine vom Kanal abhängigen Gerätefunktionen eingeht. Die in einem Kanal gemessenen Ladungsmenge hängt deshalb über die Beziehung

$$
Q=G_{0} v^{2} \sum_{s} e_{s} F_{s}
$$

von den Werten $F_{s}$ der über die Winkel integrierten Geschwindigkeitsverteilungen der Ionen $s$ bei der zentralen Geschwindigkeit $v$ des Kanals ab. Die mit den Gerätefunktionen 
(a)

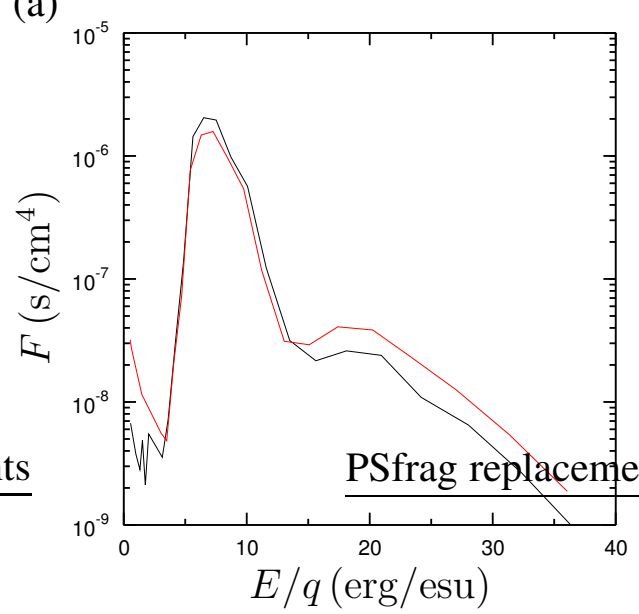

(b)

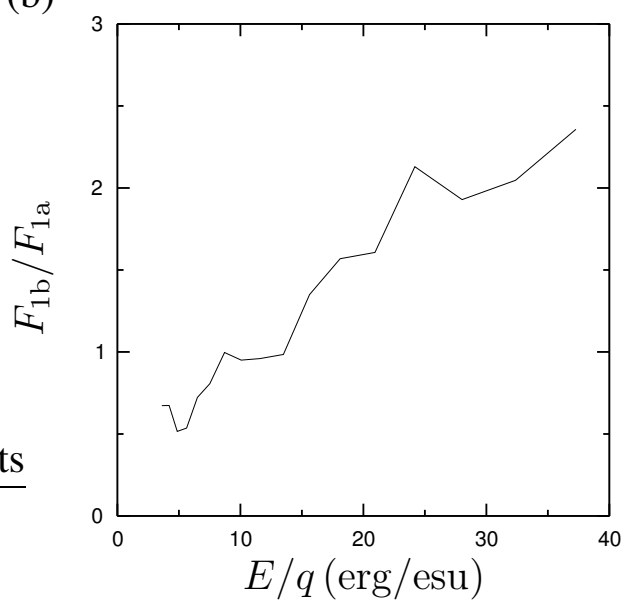

Abbildung 3.2: (a) Die von den Instrumenten 1a (schwarz) und 1b (rot) auf Helios 2

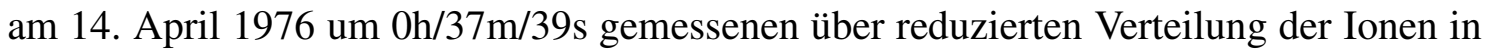
Abhängigkeit von der spezifischen Energie und (b) die mittlere Ladungszahl.

zusammenhängende Konstante $G_{0}$ wurde durch eine Eichung des Instrumentes bestimmt (s. Rosenbauer et al. 1981, S. 179f.). Durch $e_{s}$ wird die Ladung der Ionenart $s$ bezeichnet. Die Elektronik des Instrumentes zählte die Ladung als Vielfaches der Quantisierungseinheit $Q_{0}=1000 e=4.8 \times 10^{-7}$ esu. Für die Zählrate $Z$ in einem Energiekanal gilt also

$$
Z=G_{0} \frac{e}{Q_{0}} v^{2} \sum_{s} Z_{s} F_{s}
$$

berechnet werden, wobei $Z_{s}$ die Ladungszahl der Ionenart $s$ sein. Die Abbildung 3.2a zeigt die durch die Gleichung 3.5 bestimmte Ladungsgewichtung der über die Winkel integrierten Verteilungen der Ionen. Auch in dieser Verteilung ist sowohl das den Protonen zuzuordnende Hauptmaximum als auch das Nebenmaximum bei höherer spezifischer Energie zu erkennen. Das Verhältnis der von den Instrumenten 1b und 1a bestimmten reduzierten Verteilungen der Ionen gibt die mittlere Ladungszahl der in einem Energiekanal detektierten Teilchen an. Damit hilft es bei der Unterscheidung der Ionenarten, die von den einzelnen Instrumenten $1 \mathrm{a}$ und $1 \mathrm{~b}$ nicht getrennt werden konnten. In der Abbildung 3.2b ist dieses Verhältnis für das Beispiel der Abbildung 3.2a aufgetragen. Die Ionen des Hauptmaximums besitzen eine mittlere Ladungzahl von $Z \approx 1$, weshalb sie wegen ihrer Häufigkeit nur Protonen sein können. Im Bereich des Nebenmaximums beträgt die mittlere Ladungszahl $Z \approx 2$. Da Helium in allen Schichten der Sonne das zweithäufigste Element ist, darf unterstellt werden, daß es sich bei diesem Nebenmaximum im Sonnenwind um $\alpha$-Teilchen handelt.

\section{Instrument 2}

Anders als das Instrument 1a in Bezug auf die Ionen, konnte das Instrument 2 einfallende Elektronen nicht nach ihrer Elevation getrennt zählen. Es bestand aus einem halbkugelförmigen elektrostatischen Analysator, in dessen Fokus ein Teilchendetektor ange- 


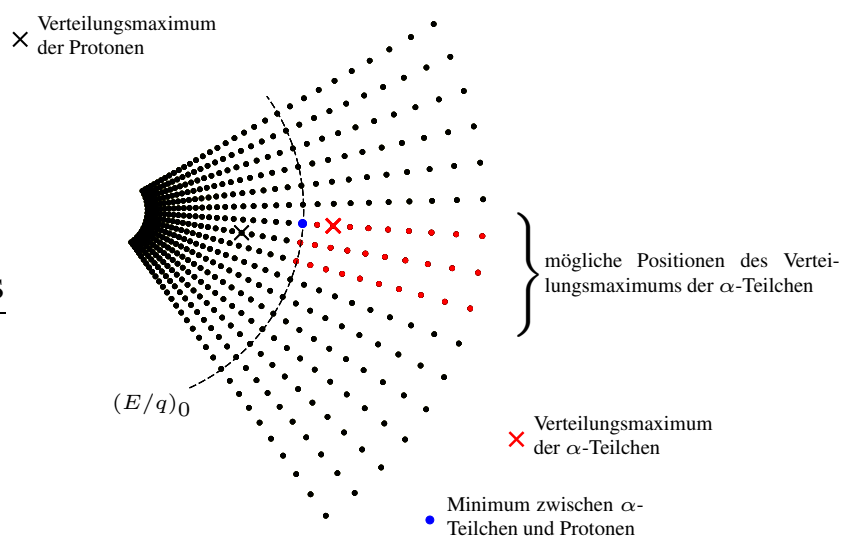

Abbildung 3.3: Skizze der Abtrennung der $\alpha$-Teilchen aus dem dreidimensionalen Zählratenspektrum des Instrumentes 1a in einer Ebene konstanter Elevation.

bracht war. Die Abhängigkeit der Elektronenverteilung vom Azimut wurde auch durch die Eigenrotation der Sonde aufgelöst. Der Öffnungswinkel des Instrumentes reichte in der Elevation von $-10^{\circ}$ bis $10^{\circ}$. Das Instrument 2 besaß 16 Energiekanäle und 8 Azimutkanäle. Die genauen Lagen der Energie- und Azimutkanäle des Instrumentes sind in dem Forschungsbericht von Rosenbauer et al. (1981) auf der S. 184 tabelliert. Die Azimutkanäle waren in guter Näherung äquidistant verteilt und ihr Abstand betrug $45^{\circ}$. Die Kanalbreiten waren aber deutlich geringer. Die Abstände der Energiekanäle erhöhten sich beginnend mit ungefähr $6.8 \times 10^{-12} \mathrm{erg}$ bei dem niedrigsten Energiekanal schrittweise um den Faktor 1.4. Die genaue Winkelausrichtung des Kanalgitters hing auch beim Instrument 2 vom wechselnden Meßmodus ab. Der Wert der Verteilungsfunktion der Elektronen bei der zentralen Geschwindigkeit eines Kanals $f$ ergibt sich aus der gemessenen Zählraten $Z$ über die Beziehung

$$
Z=G_{0} v^{4} f
$$

(vgl. 3.1.1, Instrument 1a), wobei $v$ der Betrag der Geschwindigkeit zum betreffenden Kanal ist. Die Konstante $G_{0}$ ergab sich aus einer Eichung des Instrumentes (s. Rosenbauer et al. 1981, S 182ff.).

\subsubsection{Abtrennung der $\alpha$-Teilchen}

In dieser Arbeit gehe ich von der Annahme aus, daß es sich bei den Teilchen des in der Abbildung 3.2a erkennbaren Nebenmaximums um $\alpha$-Teilchen handelt, was durch die gewichtete Ladungszahl der Ionen im Nebenmaximum gestützt wird (s. Abbildung 3.2b). Das dreidimensionale Zählratenspektrum des Instrumentes 1a soll entsprechend in zwei den Protonen und $\alpha$-Teilchen zuzuordnende Bestandteile zerlegt werden. Ich setze dafür voraus, daß die $\alpha$-Teilchen ihr Verteilungsmaximum bei einer Geschwindigkeit besitzen, die größer oder gleich derjenigen der Protonen ist, und daß ihr Verteilungsmaximum in einem der Winkelkanäle liegt, die unmittelbar an den Winkelkanal des Verteilungsmaximums der Protonen angrenzen. Besäßen die $\alpha$-Teilchen ihr Verteilungsmaximum bei 
(a)

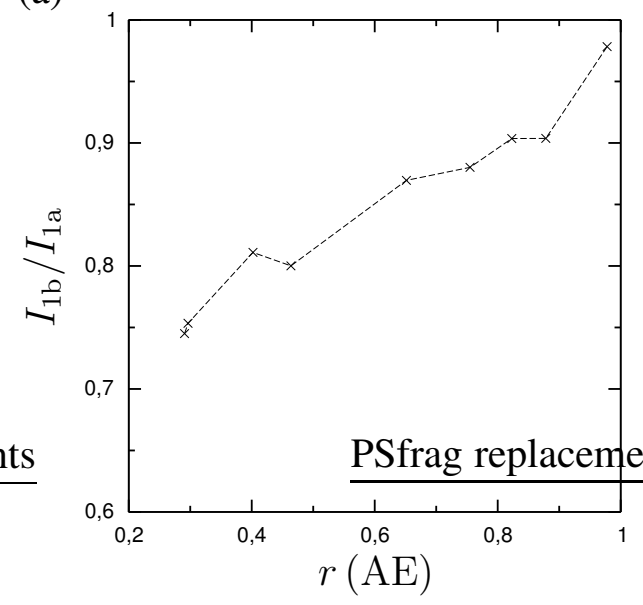

(b)

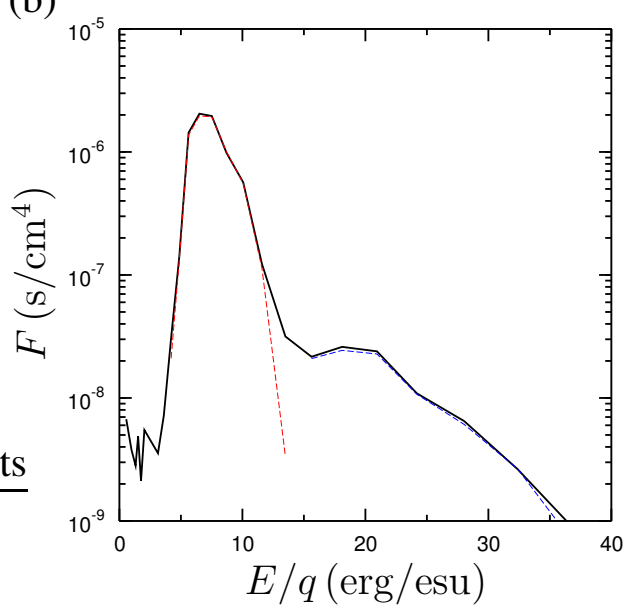

Abbildung 3.4: (a) Der Mittelwert des Verhältnisses aus den Integralen der von den Instrumenten 1a und $1 \mathrm{~b}$ bestimmten reduzierten Verteilungen. (b) Die aus den dreidimensionalen Zählratenspektren der Ionen (schwarz), der Protonen (rot) und $\alpha$-Teilchen (blau) gewonnenen reduzierten Verteilungen als Funktion der spezifischen Energie des Kanals. Die verwendeten Daten wurden auf Helios 2 am 14. April 1976 um 0h/37m/39s vom Instrument 1a gemessen (vgl. Abbildungen 3.2a und 3.2b).

der gleichen Geschwindigkeit wie die Protonen, dann gölte für die spezifischen Energien der Teilchen in den jeweiligen Maxima $(E / q)_{\alpha}=2 \cdot(E / q)_{p}$. Das Nebenmaximum liegt bei größerer spezifischer Energie, weshalb die $\alpha$-Teilchen schneller strömen müssen als die Protonen. Das Verteilungsmaximum der $\alpha$-Teilchen wird in den Energiekanälen mit $E / q \geq 2 \cdot(E / q)_{p, \max }$ und den unmittelbar an das Verteilungsmaximum der Protonen angrenzenden Winkelkanälen gesucht. Die Aufteilung des Spektrums soll sich an einem festen Energiekanal $(E / q)_{0}$ orientieren. Diesen Energiekanal wähle ich so, daß er bei dem Winkelkanal des Verteilungsmaximums der $\alpha$-Teilchen das Verteilungsminimum zwischen den Protonen und $\alpha$-Teilchen markiert. Demnach betrachte ich alle Teilchen, die bei größeren spezifischen Energien gezählt wurden als $\alpha$-Teilchen und die verbleibenden als Protonen (s. Abbildung 3.3). Um nicht versehentlich ein möglicherweise existierendes zweites Verteilungsmaximum der Protonen als $\alpha$-Teilchen abzutrennen, akzeptiere ich ein Nebenmaximum nur dann als $\alpha$-Teilchen, wenn der Energiekanal, in dem es liegt, eine mittlere Ladungszahl oberhalb von 1.5 aufweist (s. Abschnitt 3.1.1). Die Trennung findet nicht in jedem Winkelkanal bei $(E / q)_{0}$ statt. Da es möglich ist, daß die Kurve $(E / q)_{0}=$ const. bei randlagigen Winkelkanälen einen bedeutenden Teil der Verteilung der Protonen durchtrennt, erhöhe ich in jedem Winkelkanal den Grenzkanal ausgehend von $(E / q)_{0}$ um maximal zwei weitere Kanäle, sofern die Zählrate dabei abnimmt. Vor der Verwendung der Daten des Instrumentes $1 \mathrm{~b}$ für die beschriebene Abtrennung ist zu beachten gewesen, daß sich die Empfindlichkeit des Instrumentes als abhängig von der Temperatur der Sonde, die von der Temperatur des umgebenden Sonnenwindes und der Einstrahlung beeinflußt wird, erwiesen hat (Rosenbauer et al. 1981, S. 127f.). Die Empfindlichkeit nimmt mit wachsender Temperatur und somit größerer Nähe zur Sonne ab, wodurch die Berechnung der mittleren Ladungszahl verfälscht wird. Aus diesem Grund 
habe ich die mit den Instrumenten 1a und $1 \mathrm{~b}$ bestimmten über die Winkel integrierten Verteilungen der Ionen in einem gemeinsamen Bereich um das Hauptmaximum durch die Trapezregel integriert. Dort wurden nur Protonen gemessen und die Integrale $I_{1 \mathrm{a}}$, $I_{1 \mathrm{~b}}$ müssen übereinstimmen. Die Abbildung 3.4a zeigt den Verlauf des Verhältnisses aus diesen Integralen als Funktion der Entfernung zur Sonne. Es sind die Mittelwerte an ausgewählten Tagen (s. Abschnitt 3.2) zu sehen. Es ist zu erkennen, daß das Verhältniß mit geringer werdender Entfernung entsprechend der Empfindlichkeit des Instrumentes $1 \mathrm{~b}$ abnimmt. Ich habe dies in jedem Fall durch die Renormierung der Daten des Instrumentes $1 \mathrm{~b}$ korrigiert und die Gleichheit der Integrale erzwungen. Die Abbildung 3.4b zeigt das Ergebnis der Abtrennung. Die Zählraten der Protonen und $\alpha$-Teilchen sind über die Winkelkanäle summiert und durch die Gleichung 3.3 in die reduzierte Verteilung überführt worden.

\subsubsection{Das Sondenpotential und die Elektronen-Messung}

Die Berechnung der Geschwindigkeitsverteilungen der Ionen und Elektronen erfolgt über die von den Instrumenten gemessenen Teilchenströme. Diese Teilchenströme sind durch den Einfluß der Sonde auf den umgebenden Sonnenwind gestört. Die Sonde ist im Sonnenwind einem Ladungsstrom auf ihre Oberfläche ausgesetzt. Zugleich findet durch die UV-Strahlung der Sonne auf der Sondenoberfläche Photoemission von Elektronen statt. Unter diesen Ladungsströmen lädt sich die Sonde bis zu einem Gleichgewicht unter den Strömen auf. Da die Photoemission überwiegt, besitzt die Sonde ein positives Potential. In ihrem Umfeld bilden sich zudem Raumladungen aus. In dem elektrischen Feld werden Ionen und Elektronen, bevor sie in die Instrumente gelangen, gestreut. Unter Vernachlässigung von Teilchenkollisionen und der Welle-Teilchen-Wechselwirkung sind ihre Geschwindigkeitsverteilungen entlang der Teilchentrajektorien in diesem Feld konstant, d.h. es gilt

$$
\begin{aligned}
\tilde{f}(t, \vec{x}, \vec{v}) & =\tilde{f}\left(t-\tau, \vec{x}_{-\tau}, \vec{v}_{-\tau}\right) \\
& \approx f\left(t, \vec{x}, \vec{v}_{-\tau}\right)
\end{aligned}
$$

wobei $\vec{x}, t$ den Ort und die Zeit der Messung und $\widetilde{f}$ die durch die Sonde gestörte Geschwindigkeitsverteilung bezeichnen. Diese Beziehung gilt für Teilchen, deren kinetische Energie so groß ist, daß sie sich mit wachsendem zeitlichen Rückschritt $\tau$ beliebig weit von der Sonde entfernen und die Störung verlassen. Auf der räumlichen und zeitlichen Skala, die sie dabei durchqueren, darf die ungestörte Verteilung im Sonnenwind als homogen und stationär angenommen werden. Die Bahnen von Teilchen mit zu niedrigen kinetischen Energien führen auf die Sonde zurück. Bei solchen Energien können nur Teilchen gemessen werden, die wie Photoelektronen auf der Sondenoberfläche erzeugt wurden.

Isensee (1977), Isensee und Maaßberg (1981) und Voigt et al. (1981) untersuchten die Teilchenstreuung im Umfeld der Helios-Sonden. Sie fanden, daß Elektronen mit kinetischen Energien oberhalb von $20 \mathrm{eV}$ vor ihrer Messung keine merkliche Änderung der Richtung ihrer Geschwindigkeit erfuhren. Die kinetischen Energien der Ionen sind groß gegenüber ihrer möglichen Änderung im elektrischen Potential der Sonden, weshalb sie 
(a)

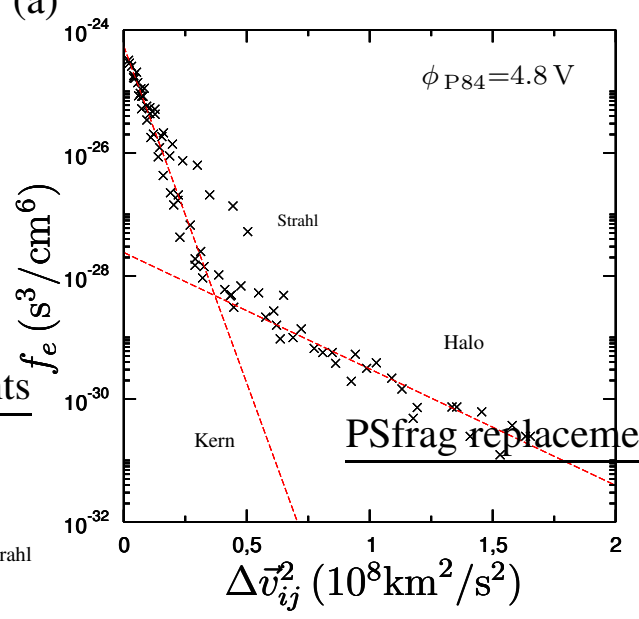

(b)

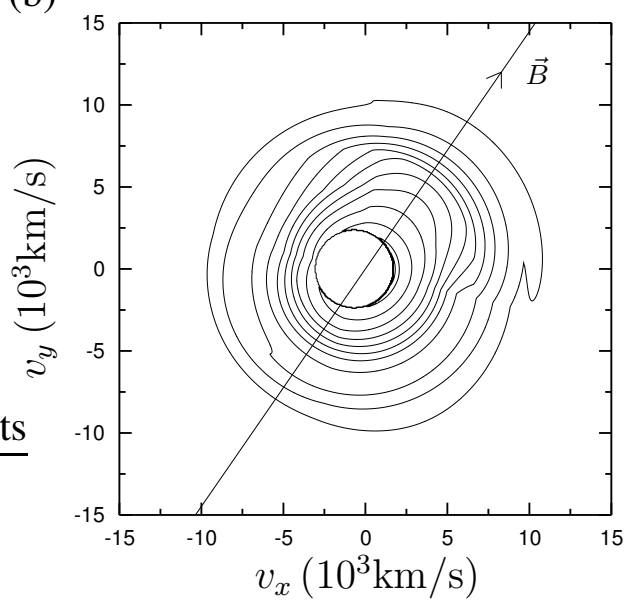

Abbildung 3.5: (a) Werte der auf Helios 2 am 4. April um 0h/26m/2s gemessen Geschwindigkeitsverteilung der Elektronen als Funktion des Betrages der Differenzgeschwindigkeit $\Delta \vec{v}_{i j}=\vec{v}_{i j}-\vec{v}_{p}$ im Kanal $i j$ nach der Korrektur um das Sondenpotential $\phi_{\mathrm{P} 84}$. Durch $\vec{v}_{p}$ wird die mittlere Geschwindigkeit der Protonen bezeichnet. (b) Höhenlinien einer einfachen und durch polynomiale Interpolation des Logarithus der in (a) gezeigten Werte gewonnenen Darstellung der Geschwindigkeitsverteilung der Elektronen (s. Fußnote 1 im Kapitel 4). Die Höhenlinien entsprechen den Anteilen 0.9, 0.7, 0.5, 0.3, 0.1, $3 \times 10^{-2}$, $10^{-2}, \ldots, 3 \times 10^{-5}$ und $10^{-5}$ des maximalen gemessenen Wertes. Die Aussparung in der Mitte der Verteilung entsteht durch die Vernachlässigung aller bei Geschwindigkeiten unterhalb von $20 \mathrm{eV}$ gemessenen Werte (s. Text). Die Abszisse zeigt in die Richtung der mittleren Geschwindigkeit der Protonen und der Ursprung stimmt mit ihr überein.

als unbeeinflußt betrachtet werden dürfen. Die zu berücksichtigende Änderung der kinetischen Energie der Elektronen ergibt sich aus der Energieerhaltung im statischen elektrischen Feld

$$
\vec{v}_{0}^{2}=\vec{v}^{2}-\frac{2 e}{m_{e}} \phi
$$

wobei $\vec{v}_{0}, \vec{v}$ die Geschwindigkeiten eines Elektrons vor und nach der Streuung und $\phi$ das elektrische Potential an der Sondenoberfläche sind. Es gilt somit

$$
f_{e}\left(t, \vec{x}, \vec{v}_{0}\right) \approx \tilde{f}_{e}\left(t, \vec{x}, \sqrt{\vec{v}_{0}^{2}+\frac{2 e}{m_{e}} \phi} \frac{\vec{v}_{0}}{\left|\vec{v}_{0}\right|}\right) .
$$

oberhalb kinetischer Energien von $20 \mathrm{eV}$. Die kinetische Energie der aus dem Sonnenwind stammenden Elektronen übersteigt deshalb bei der Messung den Grenzwert $e \phi$. Unterhalb dieser Energie können nur Photoelektronen gemessen werden. Sie besitzen eine von den Elektronen des Sonnenwindes verschiedene Energieverteilung und erlauben so prinzipiell die Bestimmung des Sondenpotentials (Rosenbauer 1973). Pilipp et al. (1984) haben für ausgewählte Daten des Instrumentes 2 auf Helios das Sondenpotential bestimmt. Sie fanden die empirische Beziehung

$$
\phi_{\mathrm{P} 84}=3.85\left(2.13-\ln \left(n_{p} r^{2} \mathrm{~cm}^{3} / \mathrm{AE}^{2}\right)\right) .
$$

Die Abnahme des Potentials mit $n_{p} r^{2}$ erklärt sich durch die Zunahme der Photoemission in größerer Nähe zur Sonne und durch die Zunahme des Zustroms negativer Ladung 
(a)

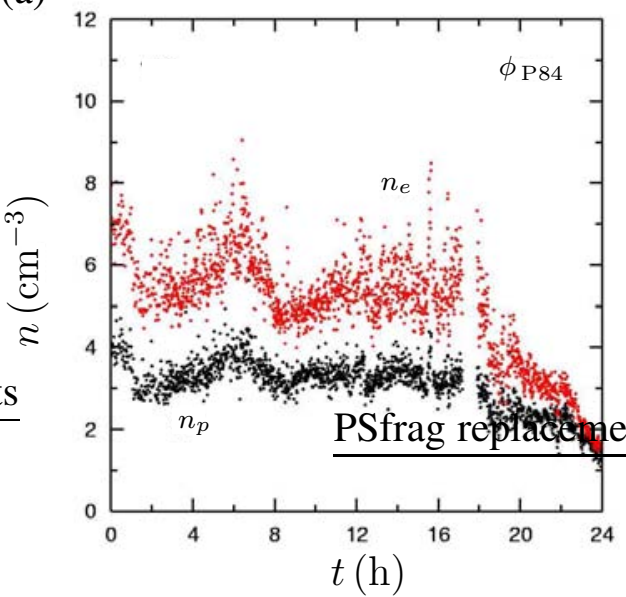

(b)

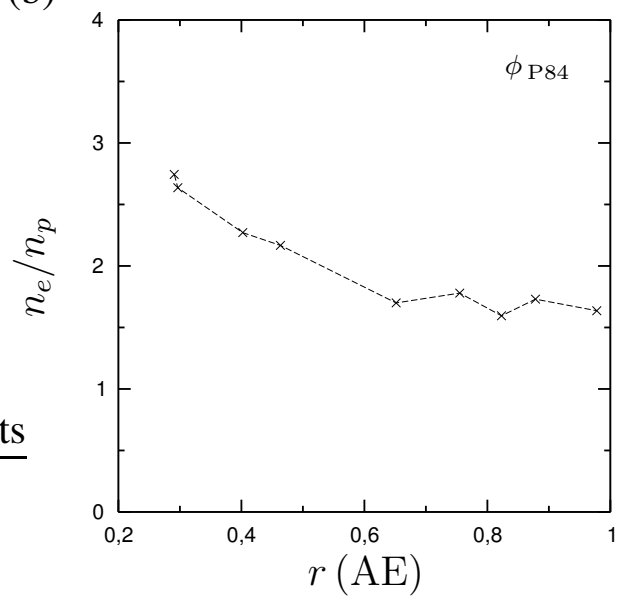

Abbildung 3.6: (a) Teilchendichten der Protonen und Elektronen am 27. Februar 1976 als Funktion des Zeitpunktes der Messung. (b) Mittelwerte des Verhältnisses des Teilchendichten der Elektronen und Protonen in ausgewählten Strömungen schnellen Sonnenwindes (s. Abschnitt 3.2) als Funktion der mittleren Entfernung der Messungen zur Sonne. Die Elektronenmessungen zu (a) und (b) sind um das Sondenpotential $\phi_{\text {P84 }}$ korrigiert worden.

auf die Sonde mit steigender Elektronendichte des Sonnenwindes. Für die Auswertung der Messungen des Instrumentes 2 gehe ich davon aus, daß die elektrische Ladungs- und Stromdichte überall verschwinden, d.h. unter Vernachlässigung der $\alpha$-Teilchen und anderer auf Helios nicht gemessener Minoritätsionen stimmen die Teilchendichten und mittleren Geschwindigkeiten der Elektronen und Protonen überein. Die Abbildung 3.5a zeigt die vom Instrument 2 gemessenen Werte einer beispielhaften Elektronenverteilung. Die Geschwindigkeiten zu den Kanälen wurden durch die Gleichung 3.8 um das Sondenpotential 3.10 korrigiert und Kanäle mit Energien unterhalb von $20 \mathrm{eV}$ blieben unberücksichtigt. Es ist zu erkennen, daß die Verteilung in zwei Bereichen, dem Kern und Halo der Verteilung, nahezu mit einer Maxwell-Verteilung übereinstimmt. Nur entlang eines einzigen Richtungskanals zeigt sich eine Abweichung davon, was auf einen begrenzten Elektronen-Strahl zurückzuführen ist (Pilipp et al. 1987a). Die gezeigte Form der Verteilung ist repräsentativ. In dieser Arbeit modelliere ich die Elektronen deshalb durch zwei bei der mittleren Geschwindigkeit der Protonen zentrierte Maxwell-Verteilungen, deren Parameter ich, wie es in der Abbildung 3.5a durch die gestrichelten Geraden dargestellt ist, durch lineare Regression bestimme.

Eine systematische Auswertung der Daten des Instrumentes 2 offenbarte einen drastischen Widerspruch zum Verschwinden der elektrischen Ladungsdichte. Die Abbildung 3.6a zeigt den zeitlichen Verlauf der aus der Kern-Halo-Darstellung berechneten Dichte der Elektronen für die auf Helios 2 am 27. Februar 1976 gemessenen Verteilungen. Die Dichte der Protonen ist auch dargestellt. Das Verhältnis der Dichten ist in diesem Zeitraum nahezu konstant, jedoch deutlich größer als eins. Solche Abweichung von der Neutralität sollten in einem zeitlichen Mittel auf der hier betrachteten Skala nicht sichtbar sein. Ladungstrennungen führen zu Fluktuationen auf der Zeitskala der Plasmafrequenz $\omega_{p e}^{2}=4 \pi e^{2} n_{e} / m_{e}$, die mit typischerweise $10^{5} \mathrm{~s}^{-1}$ sehr klein ist. Voigt et al. (1981) werte- 
(a)

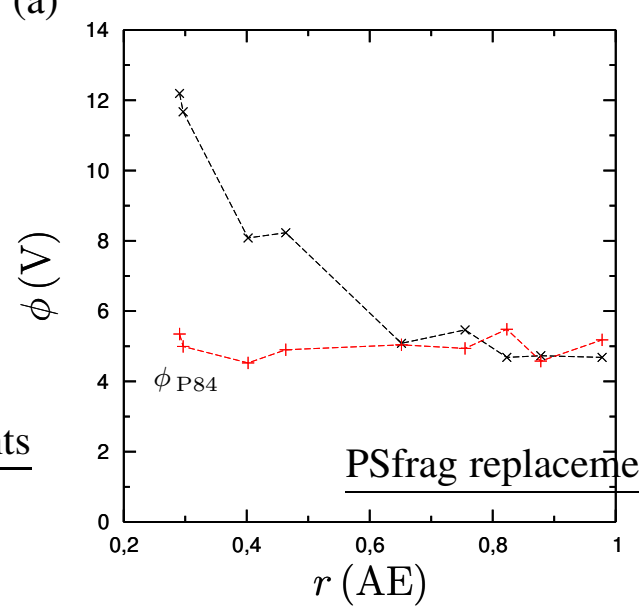

(b)

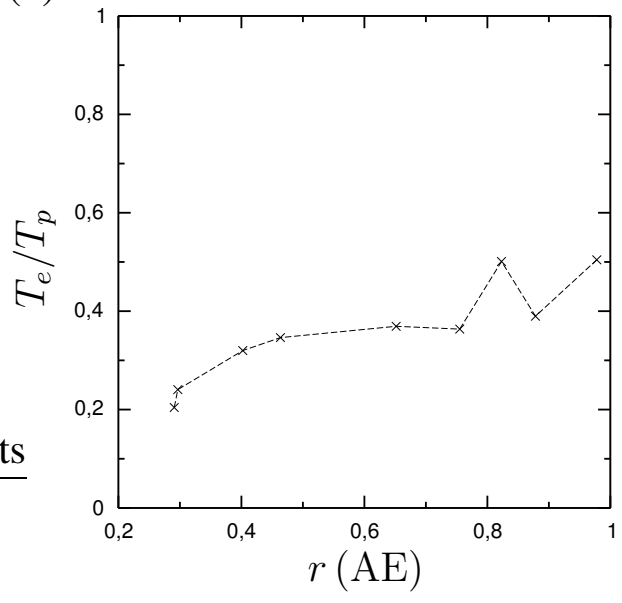

Abbildung 3.7: (a) Mittelwerte des optimierten Sondenpotentials und des Potentials $\phi_{\mathrm{P} 84}$ in ausgewählten Strömungen schnellen Sonnenwindes als Funktion der mittleren Entfernung der Messungen zur Sonne. (b) Mittelwerte des Verhältnisses der Temperaturen der Elektronen und Protonen in den ausgewählten Sonnenwindströmungen. Die Elektronenmessungen zu (b) sind durch das optimierte Sondenpotential korrigiert worden.

ten einzelne Messungen des Instrumentes 2 unter Verwendung der Korrektur 3.9 aus und fanden ebenfalls abweichende Dichten, deren Verhältnis sie als ungefähr 1.5 angaben, was mit den von mir im Aphel von Helios berechneten Werten vergleichbar ist. In der Abbildung 3.6b ist der Mittelwert des Verhältnisses in ausgewählten Strömungen schnellen Sondenwindes (s. Abschnitt 3.2) gegen die mittlere Entfernung der Messungen zur Sonne aufgetragen. Es ist ein Anstieg mit wachsender Nähe zur Sonne zu beobachten. Es muß geschlußfolgert werden, daß entweder die Eichung des Instrumentes 2 ein nicht zutreffendes nulltes Moment der Gerätefunktion ergeben hat oder die Korrektur der Geschwindigkeitsverteilung gemäß der Gleichung 3.9 und dem Sondenpotential 3.10 nicht ausreicht. Durch größere Sondenpotentiale als 3.10 kann die Elektronendichte gesenkt werden. Um eine übereinstimmung mit der Dichte der Protonen zu erzielen, müßte es für das in der Abbildung 3.5 gezeigte Beispiel 15.2 V betragen und für die meisten Daten aus dem Perihel sogar deutlich größer als $20 \mathrm{~V}$ sein. Bei den entsprechenden Energien $e \phi$ ist in den gemessenen Verteilungen aber keine Trennlinie zu den Photoelektronen zu beobachten. Diese Tatsache ließe sich durch Raumladungen im Umfeld der Sonde erklären. Die kinetische Energie, unterhalb derer ein Elektron von der Sondenoberfläche stammen muß, hängt in diesem Fall von der Einfallsrichtung der Elektronen $a^{3}$. Das Instrument 2 sammelte Elektronen zwischen den Elevationen $\pm 10^{\circ}$, so daß die scharfe Trennung zwischen den Photoelektronen und den Elektronen des Sonnenwindes verwischt (Rosenbauer 1973). Solche unter schrägem Winkel mit größeren kinetischen Energien als $e \phi$ einfallende Photoelektronen beeinflußten die Elektronendichte zusätzlich, wenn sie die von mir berücksichtigten Energiekanäle oberhalb $20 \mathrm{eV}$ noch beträfen. Und die meisten der im Kern der Verteilung für eine lineare Regression verwendeten Werte stammen aus den ersten beiden Energiekanälen oberhalb dieser Energie. Andererseits schlossen Isen-

\footnotetext{
${ }^{3}$ vgl. Bewegung eines Teilchens mit nicht verschwindendem Drehimpuls im Debye-Potential.
} 
see (1977), Isensee und Maaßberg (1981) und Voigt et al. (1981) die implizierte Richtungsänderung der Geschwindigkeit der Elektronen bei solchen Energien für Sondenpotentiale der Größenordnung von ungefähr $5 \mathrm{~V}$, die aus der Beziehung $3.10 \mathrm{im}$ schnellen Sonnenwind folgt, aus. Außerdem sollte eine Abhängigkeit vom Azimut, d.h. der Blickrichtung des Instrumentes innerhalb der Ekliptik bestehen. Die Abbildung 3.5b, in der die Höhenlinien einer beispielhaften Geschwindigkeitsverteilung von Elektronen dargestellt sind, zeigt hingegen keine deutlichen Abweichungen von der Symmetrie der Verteilung bezüglich der Richtung des magnetischen Feldes. Eine Unterschätzung des Sondenpotentials durch die Beziehung 3.10 kann somit nicht die alleinige Erklärung sein.

In dieser Arbeit habe ich mich dazu entschieden, den Dichteunterschied ebenso wie Voigt et al. (1981) als systematischen und konstanten Fehler des Instrumentes zu betrachten. Den Anstieg des Dichteverhältnisses mit der Nähe zur Sonne führe ich darauf zurück, daß die Abhängigkeit des Sondenpotentials vom Produkt $n_{p} r^{2}$ nicht zutreffend ist. Der Anstieg der Häufigkeit der Photoemission muß den Anstieg der Elektronendichte und des verbundenen Zustroms negativer Ladung auf die Sondenoberfläche überwiegen und zu einem größeren Sondenpotential als das der Beziehung 3.10 führen. Aus diesem Grund wende ich in einem ersten Schritt den Mittelwert des am 23. Januar, 19. und 27. Februar 1976 (s. Tabelle 3.1) im Umfeld des Aphels von Helios gemessen Verhältnisses aus der Dichte der Elektronen und Protonen, $\overline{n_{e} / n_{p}}=1.69$, als Korrekturfaktor zur Eichung des Instrumentes auf alle Meßwerte an. In einem zweiten Schritt bestimme ich das Sondenpotential so, daß sich nach der Transformation der Geschwindigkeiten durch die Energieerhaltung 3.8 bzw. der Verteilung durch die Gleichung 3.9 eine mit der Protonendichte übereinstimmende Dichte der Elektronen ergibt. Die Abbildung 3.7a zeigt den an ausgewählten Tagen auf diese Weise berechneten Mittelwert des Sondenpotentials im Vergleich zum Mittelwert des nach Pilipp et al. (1984) berechneten Potentials als Funktion der mittleren Entfernung der Messungen zur Sonne. Während das Sondenpotential von Pilipp et al. (1984) im Mittel konstant bleibt, steigt das den Daten angepaßte erwartungsgemäß an und ist nur in der Nähe des Aphels von Helios von der gleichen Größenordnung. In der Abbildung 3.7b ist der Mittelwert des Verhältnisses der Temperaturen der Elektronen und Protonen als Funktion der Entfernung zur Sonne aufgetragen. Die Elektronentemperatur ist um einen Faktor 2 bis 4 kleiner als diejenige der Protonen, was mit den Ergebnissen von Pilipp et al. (1987a), Pilipp et al. (1987b) übereinstimmt.

\subsection{Datenauswahl}

Für die Untersuchungen in dieser Arbeit stehen prinzipiell alle Einzelmessungen des Plasmaexperimentes auf Helios 1 und 2 zur Verfügung. Die außerhalb der Primärphasen von Helios 1 und 2 schnell geringer werdende Datendichte schränkt die Datenauswahl allerdings auf diese Phasen der Mission ein. Zudem verwende ich aus Gründen der Datenqualität ausschließlich die Daten des Plasmaexperimentes auf Helios 2. Die Abbildung 3.8 zeigt die aus den Daten des Instrumentes 1a auf Helios 2 innerhalb der Primärphase gewonnene Dichte und mittlere radiale Geschwindigkeit der Protonen als Funktion des Zeitpunkts der Messung. In diesem Zeitraum bewegte sich die Sonde von 0.98 AE unmittelbar nach dem Beginn der Messungen bis zu ihrem ersten Periheldurchgang bei $0.29 \mathrm{AE}$ am 


\begin{tabular}{rlccccc}
\hline \hline \multicolumn{1}{c}{ Tag } & Datenanzahl & $\bar{r}(\mathrm{AE})$ & $\bar{B}\left(10^{-5} \mathrm{G}\right)$ & $\overline{n_{p}\left(\mathrm{~cm}^{-3}\right)}$ & $\overline{v_{r p}}(\mathrm{~km} / \mathrm{s})$ \\
\hline 23. & Januar & $2031(1196)$ & 0.98 & 5.5 & 2.4 & 694 \\
19. & Februar & $1926(1308)$ & 0.88 & 7.4 & 3.3 & 640 \\
27. & Februar & $1906(1356)$ & 0.82 & 7.4 & 3.1 & 683 \\
6. & März & $1949(1567)$ & 0.75 & 9.6 & 4.1 & 678 \\
16. März & $2013(1288)$ & 0.65 & 11.1 & 5.3 & 615 \\
4. April & $1012(771)$ & 0.40 & 23.6 & 16.2 & 608 \\
14. April & $1984(1310)$ & 0.30 & 42.2 & 26.2 & 672 \\
16. April & $2042(1282)$ & 0.29 & 42.6 & 25.1 & 704 \\
\hline 6. April 1977 & $1916(724)$ & 0.46 & 19.6 & 11.1 & 682 \\
\hline \hline
\end{tabular}

Tabelle 3.1: Die Anzahl der an dem jeweiligen Tag des Jahres 1976 verfügbaren Datensätze von Helios 2 (in der Klammer ist die tatsächlich in den Untersuchungen der Kapitel 6 und 7 verwendeten Anzahl von Datensätzen angegeben, s. Text) sowie die Tagesmittel des Betrages des magnetischen Feldes, der Dichte und der radialen Geschwindigkeit der Protonen.

Tag 107. Es sind wiederkehrende Strömungen schnellen Sonnenwindes mit Geschwindigkeiten von oberhalb $550 \mathrm{~km} / \mathrm{s}$ zu erkennen. Diese Ereignisse überdauern jedesmal einen oder mehrere Tage und umfassen dabei 87504 von insgesamt 189185 Einzelmessungen des Zeitraums. Aus diesen Ereignissen habe ich einzelne Tagesdatensätze so ausgewählt, daß zum einen keine Anteile langsameren Sonnenwindes enthalten sind und zum anderen der Entfernungsbereich von 0.29 AE bis $0.98 \mathrm{AE}$ möglichst gleichmäßig unterteilt ist. Die Mittelpunkte der betreffenden Tage sind in der Abbildung 3.8 durch gestrichelte Geraden markiert.

Die Tabelle 3.1 gibt für jeden Tag die mittleren Werte der Dichte und der radialen Geschwindigkeit der Protonen, des magnetischen Feldes sowie die Anzahl der jeweils verfügbaren Datensätze des Plasmaexperimentes an. Die am 14./16. April 1976 vorhandenen Datensätze des Instrumentes 3 sind in den Zahlen enthalten. Die Mittelwerte weiterer Plasmaparameter sind für die ausgewählten Tage in der Tabelle A.1 zusammengetragen und der Anhang A gibt eine vollständige Übersicht der parametrischen Eigenschaften des schnellen Sonnenwindes der ausgewählten Tage. Um die Lücke zwischen den Entfernungen von 0.65 AE und 0.40 AE zu schließen, habe ich die Daten von Helios 2 vom 6. März 1977, die nicht mehr aus der Primärphase stammen, hinzugenommen. Die einzige in der Primärphase vorhandene Strömung schnellen Sonnenwindes zwischen diesen Entfernungen umfaßt mit 961 nur wenige Datensätze. Insbesondere ist die mittlere radiale Geschwindigkeit der Protonen in dieser Strömung mit $576 \mathrm{~km} / \mathrm{s}$ zu gering, und es sind auch Geschwindigkeiten unterhalb von $550 \mathrm{~km} / \mathrm{s}$ enthalten.

Die Summe aller an den ausgewählten Tagen vorhandenen Datensätze beträgt 16779. Die in den Kapiteln 6 und 7 dargestellten Ergebnisse dieser Arbeit gründen sich allerding nur auf 10804 der möglichen Datensätze. Dafür gibt es mehrere Ursachen. Erstens ließen sich 

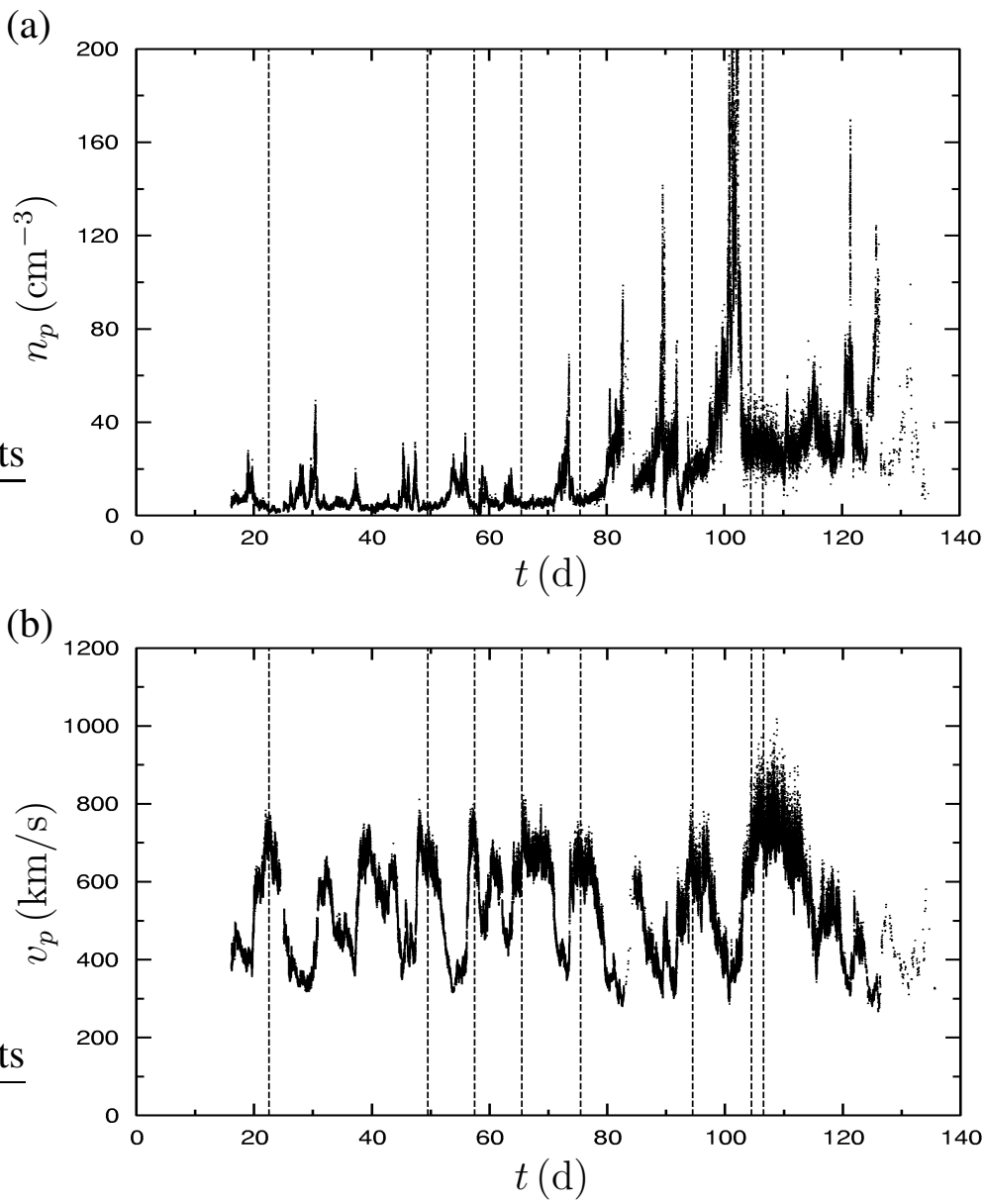

Abbildung 3.8: Die aus des Daten des Intrumentes 1a auf Helios 2 innerhalb der Primärphase gewonnene (a) Dichte und (b) radiale Geschwindigkeit der Protonen als Funktion des Zeitpunkts der Messung in Tagen.

nicht in allen Datensätzen des Instrumentes 1a die $\alpha$-Teilchen erkennen und abtrennen. Solche Meßdaten blieben unberücksichtigt. Da das Instrument 3 aufgrund seiner Konstruktion nur die Protonen maß, habe ich die Daten dieses Instrumentes auch nicht verwendet. Zweitens liegen nicht in jedem Fall zugleich die Meßdaten des Instrumentes 1a und des Instrumentes 2, d.h. der Ionen bzw. Elektronen vor. Die Anzahl der Datensätze des Plasmaexperimentes, die die gleichzeitige Bestimmung der Meßwerte der Geschwindigkeitsverteilungen der Protonen, $\alpha$-Teilchen und Elektronen ermöglichen beträgt 14142. Drittens hängt die Gültigkeit der allgemeinen Dispersionsmatrix 2.11 von der Symmetrie der Geschwindigkeitsverteilungen aller Teilchenarten um das magnetische Feld ab. Diese Symmetrie muß in einem gemeinsamen Bezugssystem, z.B. bezogen auf die Geschwindigkeit des Massenschwerpunktes, gelten. Daraus folgt, daß die Relativgeschwindigkeiten der Teilchenpopulationen parallel zum magnetischen Feld gerichtet sein müssen. Die im schnellen Sonnenwind gemessenen Relativgeschwindigkeiten $\Delta \vec{v}_{\alpha, p}$ der Protonen und $\alpha$-Teilchen erfüllen diese Eigenschaft im Mittel. Der Anteil $\Delta \vec{v}_{\alpha, p}^{\perp}$ senkrecht zum magnetischen Feld streut gehäuft um Null und sein Mittelwert verschwindet. Ich habe deshalb nur diejenigen Meßdaten in die Auswertung einbezogen, für die der Winkel zwischen der 
Relativgeschwindigkeit $\Delta \vec{v}_{\alpha, p}$ und dem magnetischen Feld kleiner als $20^{\circ}$ ist. Dadurch bleiben die genannten 10804 Datensätze übrig. 



\section{Modellierung der Geschwindigkeitsverteilungen der Protonen}

Es ist aus den in der Einleitung genannten Gründen das Ziel dieser Arbeit, den schnellen Sonnenwind mit den auf Helios gemessenen Geschwindigkeitsverteilungen der Protonen, der $\alpha$-Teilchen und Elektronen auf Mikroinstabilitäten zu untersuchen. Außerdem sollen die Verteilungen der Protonen auf die Ausbildung von Diffusionsplateaus unter dem Einfluß resonanter und sich parallel zum magnetischen Feld ausbreitender Ionen-ZyklotronWellen überprüft werden. Sowohl die Existenz und die Eigenschaften von Mikroinstabilitäten als auch die Form der Diffusionsplateaus hängen sensibel von den Geschwindigkeitsverteilungen der Protonen ab. Es ist daher erforderlich, sie anhand der Meßdaten des Instrumentes 1a so zu modellieren, daß ihre entscheidenden Eigenschaften dargestellt sind. Der parametrische Ansatz einer Maxwell-Verteilung ist dazu nicht geeignet und auch physikalisch unbegründet, da der schnelle Sonnenwind nahezu kollisionsfrei ist. Die Abbildung 4.1 zeigt die Höhenlinien einer polynomialen Interpolation ${ }^{1}$ des Logarithmus der gemessenen Werte einer exemplarischen, aus der Nähe des Perihels von Helios stammenden Verteilung der Protonen. Die Verteilung ist bis auf geringe Abweichungen symmetrisch um das magnetische Feld und besitzt eine deutliche Temperaturanisotropie, $T_{\perp} / T_{\|}>1$. Sie besitzt zudem einen Strahl von Protonen, die sich entlang des magnetischen Feldes mit größerer Geschwindigkeit als die Protonen im Kern der Verteilung von der Sonne entfernen. Die Temperaturanisotropie und der Protonenstrahl sind, wie im Abschnitt 2 beschrieben wurde, die mögliche Ursache von Mikroinstabilitäten. Diese Eigenschaften müssen, ohne im Vorfeld Annahmen über ihre Gestalt und ihr Ausmaß zu machen, durch einen geeigneten Ansatz modelliert werden. Die zur Erzeugung der Abbildung 4.1 verwendete polynomiale Interpolation und jede andere stückweise Interpolation der Meßwerte sind solche geeigneten Modelle der Geschwindigkeitsverteilungen. Für die von mir angestrebten Untersuchungen sind sie allerdings ungeeignet und würden aufgrund ihrer mathematischen Form zu prinzipiellen Schwierigkeiten im Zusammenhang mit der Definition der Dispersionsbeziehung von kinetischen Plasmawellen führen. In den folgenden Abschnitten soll der von mir verwendete Ansatz zur Modellierung der Geschwindigkeitsverteilungen und die Methode beschrieben werden, diese Modellverteilung aus den Meßwerten zu berechnen.

\footnotetext{
${ }^{1}$ in sphärischen Koordinaten $\sum_{i, j, k=0}^{3} a_{i j k} \cdot\left(v-v_{n}\right)^{i}\left(\alpha-\alpha_{m}\right)^{j}\left(\epsilon-\epsilon_{l}\right)^{k}$ um den Gitterpunkt $v_{n}, \alpha_{m}$, $\epsilon_{l}$.
} 


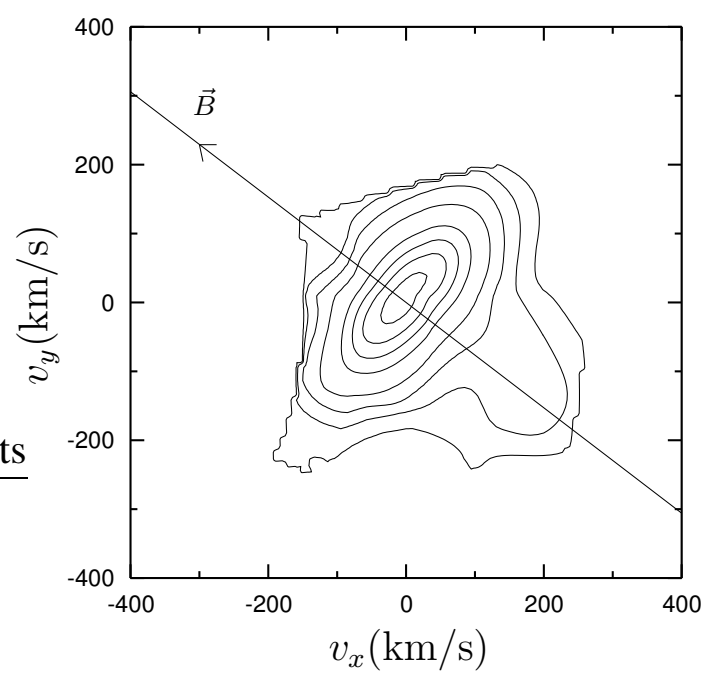

Abbildung 4.1: Höhenlinien der auf Helios 2 am 16. April 1976 um 17h/8m/31s gemessenen Geschwindigkeitsverteilung der Protonen. Ihre Werte wurden polynomial interpoliert (s. Text) und in der durch die mittlere Geschwindigkeit der Protonen und dem magnetischen Feld definierten Ebene berechnet. Die Abszisse zeigt in die Richtung der mittleren Geschwindigkeit und der Ursprung stimmt mit ihr überein. Die Höhenlinien entsprechen den Anteilen 0.9, 0.7, 0.5, 0.3, 0.1, 0.032, 0.01 und 0.0032 des Maximums der Verteilung.

\subsection{Entwicklung nach Kernfunktionen}

Die gemessenen Geschwindigkeitsverteilungen sollen so anhand der Meßdaten modelliert werden, daß keine Annahmen über ihre funktionale Form aufgestellt werden müssen. Der erforderliche Ansatz muß somit nicht-parametrisch sein. Nicht-parametrische Ansätze besitzen die Eigenschaft, im Grenzfall unendlich vieler und dicht liegender Meßpunkte gegen den Zusammenhang zu konvergieren, der gemessen wird. Die Darstellung

$$
f_{h}(\vec{v})=\sum_{i=1}^{N} \frac{F_{i}}{h^{3}} K\left(\frac{\vec{v}-\vec{v}_{i}}{h}\right)
$$

besitzt diese Eigenschaft, wobei die Koeffizienten $F_{i}=F_{i}\left(f_{1}, \ldots, f_{N}\right)$ vorerst unbestimmte und geeignete Funktionen der Meßwerte sind. Die Geschwindigkeitsverteilung wird nach Kernfunktionen entwickelt, welche in den Kanalzentren $\vec{v}_{i}$ des Meßinstrumentes positioniert sind und mit wachsendem Abstand hinreichend schnell abfallen.

Kern-Entwicklungen von Regressions- oder Modellfunktionen werden in der Literatur, die sich mit der nicht-parametrischen Regression, Dichteschätzproblemen ${ }^{2}$ oder der Mustererkennung befaßt, ausgiebig diskutiert (s. z.B. Gasser und Müller 1979, Hand 1982, Silverman 1986, Scott 1992). Und es wird untersucht, auf welche Weise sich optimale Glättungsparameter $h$ und Kernfunktionen bestimmen lassen. Die Form der Kernfunktion beeinflußt die Qualität des Ansatzes 4.1 im Vergleich zur Wahl des Glättungsparameters nur unwesentlich (s. Scott 1992, S. 133ff). Aus physikalischen Gründen ist an dieser

\footnotetext{
${ }^{2}$ Rekonstruktion von Wahrscheinlichkeitsdichten aus Zufallsmengen
} 
Stelle zu fordern, daß die verwendete Kernfunktion positiv definit ist und ihre Geschwindigkeitsmomente existieren. Ich benutze in dieser Arbeit die Gaußsche Kernfunktion

$$
K(\vec{x})=\frac{1}{(2 \pi)^{3 / 2}} e^{-\vec{x}^{2} / 2} .
$$

Sie erlaubt es, die Dispersionsbeziehungen 2.17 und 2.18 von sich parallel zum magnetischen Feld ausbreitenden kinetischen Plasmawellen für Geschwindigkeitsverteilungen der Form 4.1 analytisch anzugeben und numerisch zu lösen (s. Anhang D und Kapitel 5). Das Geschwindigkeitsintegral innerhalb der Dispersionsmatrix 2.11 läßt sich aus dem Ansatz 4.1 mit der Gaußschen Kernfunktion nicht analytisch ausführen, so daß zur Untersuchung von Instabilitäten von sich schräg ausbreitenden Wellenmoden ein einfacheres Modell der Geschwindigkeitsverteilung der Protonen nötig ist (s. Abschnitt 6.1).

\subsection{Optimale Glättungsparameter}

Ein optimaler Glättungsparameter existiert nicht in eindeutiger Weise. Die Bedeutung der Bezeichnung optimal muß, nicht frei von jeder Willkür, definiert werden. Eine Möglichkeit besteht in der Minimierung eines vorgegebenen Abstandes zwischen der zu modellierenden Geschwindigkeitsverteilung $f$ und dem Ansatz $f_{h}$ nach dem Glättungsparameter. Ein dafür geeigneter Abstand ist

$$
d\left(f, f_{h}\right)=\int d^{3} v\left|f(\vec{v})-f_{h}(\vec{v})\right|^{2} .
$$

Im allgemeinen sind die Meßwerte $f_{i}$ und somit die Entwicklungskoeffizienten $F_{i}$ und der Ansatz $f_{h}$ mit zufälligen Fluktuationen behaftet. Bei diesen fluktuierenden Anteilen handelt es sich nur in geringerem Umfang um Meßfehler des Instrumentes (s. Abschnitt 2.1). Damit der optimale Glättungsparameter diese Tatsache berücksichtigt, sollte der über die Verteilung der zufälligen Beiträge gemittelte Abstand

$$
\left\langle d\left(f, f_{h}\right)\right\rangle=\int d^{3} v\left(\left\langle f_{h}^{2}(\vec{v})\right\rangle-\left\langle f_{h}(\vec{v})\right\rangle^{2}\right)+\int d^{3} v\left|f(\vec{v})-\left\langle f_{h}(\vec{v})\right\rangle\right|^{2}
$$

betrachtet werden. Dieser Mittel- oder Erwartungswert des Abstandes wird in der Literatur mit MISE (mean integrated squared error) abgekürzt. Er setzt sich aus der integrierten Varianz der Modellverteilung $f_{h}$ und dem Abstand $d\left(f,\left\langle f_{h}\right\rangle\right)$ ihres Mittels zu der Geschwindigkeitsverteilung $f$ zusammen.

Der praktische Nutzen des MISE in der Form 4.4 ist begrenzt. Die Geschwindigkeitsverteilung $f$ ist nicht verfügbar und nur ihre Meßwerte $f_{i}$ sind bekannt. Außerdem ist die statistische Verteilung der fluktuierenden Anteile in den Meßwerten und somit des fluktuierenden Anteils des Ansatz $f_{h}$ unbekannt. Daher ist es unvermeidlich, Annahmen über die Statistik der zufälligen Anteile in den Meßwerten zu machen. Eine Möglichkeit einen optimalen aus dem MISE ohne Kenntnis der Verteilung $f$ zu bestimmen, eröffnet die asymptotische Form des MISE 4.4 im Grenzfall unendlich vieler und in einem beschränkten Gebiet dicht liegender Meßpunkte. Der asymptotische MISE verdeutlicht 
insbesondere den Einfluß der beiden Anteile des MISE 4.4 auf den optimalen Glättungsparameter. Die Koeffizienten $F_{i}$ sollen im folgenden statistisch unabhängig voneinander sein

$$
\left\langle F_{i} F_{j}\right\rangle=\left\langle F_{i}\right\rangle\left\langle F_{j}\right\rangle, \quad i \neq j
$$

und die Mittelwerte

$$
\left\langle F_{i}\right\rangle \approx V_{N} f\left(\vec{v}_{i}\right)
$$

sowie die Varianzen

$$
\left\langle F_{i}^{2}\right\rangle-\left\langle F_{i}\right\rangle^{2} \approx V_{N}^{2} \sigma\left(\overrightarrow{v_{i}}\right)
$$

asymptotisch mit dem Parameter

$$
N \cdot V_{N}=\int_{\mathcal{V}} d^{3} v 1
$$

skalieren, wobei $\mathcal{V}$ das von den Messungen ausgefüllte Gebiet und $\sigma\left(\overrightarrow{v_{i}}\right)$ durch 4.7 definiert seien (vgl. z.B. Härdle und Gasser 1984, Altman 1990). Asymptotisch stimmen die Koeffizienten im Mittel bis auf den Volumenanteil $V_{N}$ mit der gesuchten Geschwindigkeitsverteilung überein. Desweiteren muß der Glättungsparameter gegen 0 konvergieren. Unter diesen Vorausetzungen erfüllen die Varianz der Entwicklung 4.1 und der Abstand ihres Mittels zur Geschwindigkeitsverteilung asymptotisch

$$
\int d^{3} v\left(\left\langle f_{h}^{2}(\vec{v})\right\rangle-\left\langle f_{h}(\vec{v})\right\rangle^{2}\right) \approx \frac{V_{N}}{h^{3}} \int_{\mathcal{V}} d^{3} v \sigma^{2}(\vec{v}) \int d^{3} x K^{2}(\vec{x})
$$

und

$$
\int d^{3} v\left|f(\vec{v})-\left\langle f_{h}(\vec{v})\right\rangle\right|^{2} \approx \frac{h^{4}}{4}\left(\sum_{i, j} \frac{\partial^{2} f}{\partial v_{i} \partial v_{j}}(\vec{v}) \int d^{3} x x_{i} x_{j} K(\vec{x})\right)^{2} .
$$

Dafür muß die Kernfunktion normiert sein, $\int d^{3} x K=1$, und ihr erstes Moment verschwinden. Der rechten Seite der Gleichung 4.10 liegt eine Taylorentwicklung zugrunde. Alle größeren Ordnungen als $h^{4}$ wurden vernachlässigt. Für die von mir verwendete Gaußsche Kernfunktion lautet die asymptotische Form des MISE somit

$$
\left\langle d\left(f, f_{h}\right)\right\rangle \approx \frac{V_{N}}{(4 \pi)^{3 / 2} h^{3}} \int_{\mathcal{V}} d^{3} v \sigma^{2}(\vec{v})+\frac{h^{4}}{4} \int d^{3} v\left(\sum_{i} \frac{\partial^{2} f}{\partial v_{i}^{2}}(\vec{v})\right)^{2}
$$

Die von den zufälligen Anteilen der Messung herrührende asymptotische Varianz fällt mit $h^{-3} \mathrm{ab}$, während der Abstand des Mittels mit $h^{4}$ anwächst. Der optimale Glättungsparameter

$$
h^{*}=\left(\frac{(4 \pi)^{3 / 2}}{3 V_{N} \int_{\mathcal{V}} d^{3} v \sigma^{2}} \int d^{3} v\left(\sum_{i} \frac{\partial^{2} f}{\partial v_{i}^{2}}(\vec{v})\right)^{2}\right)^{-1 / 7}
$$

minimiert die Summe beider Anteile und stellt einen Ausgleich zwischen den Notwendigkeiten her, die Entwicklung 4.1 aufgrund der zufälligen Fluktuationen zu glätten und sie mit den einzelnen Meßwerten in Übereinstimmung zu bringen. Mit wachsender Anzahl 
(a)

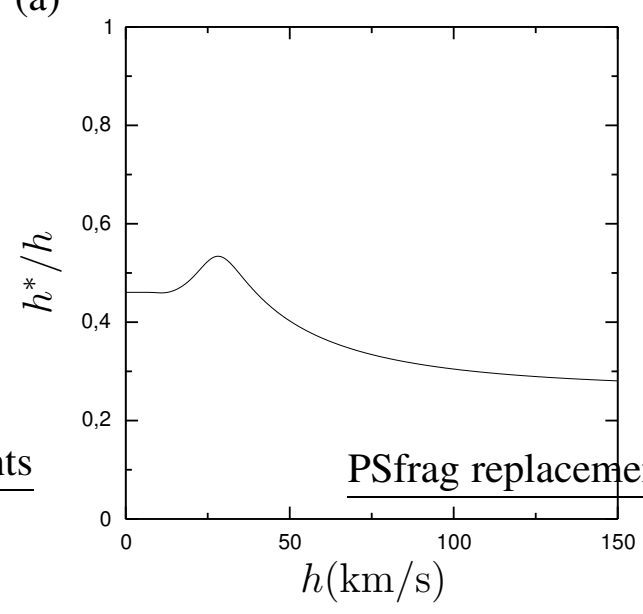

(b)

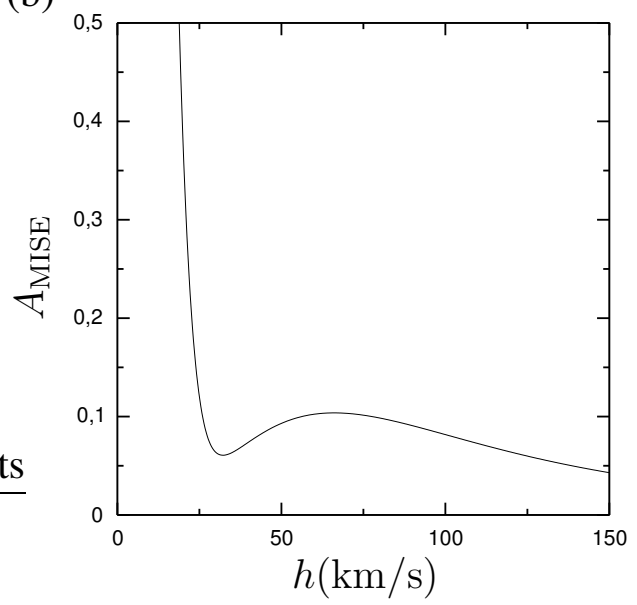

Abbildung 4.2: (a) Der optimale Glättungsparameter 4.19 als Funktion des Glättungsparameters des Ansatzes. (b) Asymptotische Form 4.18 des MISE in Abhängigkeit von dem Glättungsparameter. Die Abbildungen a und b gehören zu den Meßwerten der in der Abbildung 4.1 gezeigten Geschwindigkeitsverteilung.

der Meßwerte verschwindet die Varianz, wodurch der optimale Glättungsparameter, wie es zuvor angenommen wurde, gegen Null konvergiert.

Die asymptotische Form des MISE 4.11 enthält weiterhin die unbekannte Geschwindigkeitsverteilung $f$. Dennoch erlaubt sie es, ohne die Kenntnis von $f$ einen optimalen Glättungsparameter zu bestimmen. Scott et al. (1977) ersetzen dafür die Verteilung $f$ in dem optimalen Glättungsparameter 4.12 durch die Entwicklung 4.1 mit einem ersten Schätzwert $h_{0}$. Darin müssen die Koeffizienten $F_{i}$ nun als frei von statistischen Anteilen angenommen und direkt aus den Meßwerten berechnet werden. Dies führt iterativ zu einer Folge $h_{i+1}=h^{*}\left(f_{h_{i}}\right)$ deren Grenzwert in der Annahme, daß die Verteilung sukzessiv besser angenähert wird, als optimaler Glättungsparameter betrachtet werden darf. Dieser optimale Glättungsparameter ist somit ein Fixpunkt der Beziehung $h=h^{*}\left(f_{h}\right)$. Er ist unabhängig vom Startwert $h_{0}$, falls genau ein Fixpunkt existiert. Scott (1992) schlug alternativ vor, die Geschwindigkeitsverteilung bereits in der asymptotischen Form 4.11 des MISE durch einen Ansatz $f_{h}$ zu ersetzen und das Minimum des sich ergebenden Ausdrucks

$$
A_{\mathrm{MISE}} \equiv \frac{V_{N}}{(4 \pi)^{3 / 2} h^{3}} \int_{\mathcal{V}} d^{3} v \sigma^{2}+\frac{h^{4}}{4} \int d^{3} v\left(\sum_{i} \frac{\partial^{2} f_{h}}{\partial v_{i}^{2}}(\vec{v})\right)^{2}
$$

als optimalen Glättungsparameter zu betrachten.

\subsection{Anwendung auf die Meßdaten}

Sowohl die Iteration der Gleichung 4.12 als auch die Minimierung des asymptotischen MISE in der Näherung 4.13 bieten prinzipiell die Möglichkeit zur automatischen Bestimmung eines optimalen Glättungsparameters. Für die in dieser Arbeit verwendete Gauß- 
sche Kernfunktion gilt

$$
\begin{aligned}
\int d^{3} v\left(\sum_{i=1}^{3} \frac{\partial^{2} f_{h}}{\partial v_{i}^{2}}(\vec{v})\right)^{2} & =\frac{1}{(4 \pi)^{3 / 2} h^{7}} \\
& \times \sum_{i, j=1}^{N} F_{i} F_{j} P\left(\frac{\left|\vec{v}_{i}-\vec{v}_{j}\right|}{2 h}\right) e^{-\left|\vec{v}_{i}-\vec{v}_{j}\right|^{2} / 4 h^{2}}
\end{aligned}
$$

worin das Polynom

$$
P(x)=x^{4}-5 x^{2}+\frac{15}{4}
$$

vorkommt (s. Anhang B). Die Abhängigkeit der Koeffizienten $F_{i}$ von den Meßwerten $f_{i}$ habe ich bislang nicht definiert. Für die Berechnung des optimalen Glättungsparameters gebe ich sie durch $F_{i}=V_{N} f_{i}$ vor.

Der Beitrag der Varianz 4.7 zum asymptotischen MISE 4.13 kann allenfalls grob abgeschätzt werden. Eine Möglichkeit besteht in der nicht vollständig willkürlichen Annahme, daß die in den Kanälen des Instrumentes gemessenen Zählraten der PoissonVerteilung folgen. Jede in endlichen Zeitintervallen $\left(t_{0}, t_{1}\right)$ gemessene ganzzahlige Zufallsgröße $Z\left(t_{0}, t_{1}\right)$ ist Poisson-verteilt, wenn $Z\left(t_{0}, t_{1}\right)$ und $Z\left(t_{2}, t_{3}\right)$ für disjunkten Zeitintervalle $\left(t_{0}, t_{1}\right)$ und $\left(t_{2}, t_{3}\right)$ statistisch unabhängig sind und wenn die Wahrscheinlichkeit für das Ereignis $Z(t, t+\Delta t)=1$ in kleinen Zeitinvallen $\Delta t$ wie $\lambda \Delta t$ mit einer reellen Konstanten $\lambda$ skaliert. Die Wahrscheinlichkeit für $Z(t, t+\Delta t)>1$ muß von der Ordnung $O\left(\Delta t^{2}\right)$ sein. Der Mittelwert und die Varianz der Poisson-Verteilung stimmen überein. Der beste Näherungswert für den Mittelwert ist im Fall einer einzigen Messung die gemessene Zählrate selbst. Aus der Proportionalität zwischen den Zählraten und den Werten der Geschwindigkeitsverteilung folgen

$$
\left\langle f_{i}^{2}\right\rangle-\left\langle f_{i}\right\rangle^{2} \approx \frac{f_{i}^{2}}{Z_{i}}
$$

und letztlich

$$
\int_{\mathcal{V}} d^{3} v \sigma^{2} \approx V_{N} \sum_{i=1}^{N}\left(\left\langle f_{i}^{2}\right\rangle-\left\langle f_{i}\right\rangle^{2}\right) \approx V_{N} \sum_{i=1}^{N} \frac{f_{i}^{2}}{Z_{i}} .
$$

Die statistische Unabhängigkeit von Zählraten in beliebigen getrennten Teilintervallen des Meßzeitraumes muß allerdings bezweifelt werden, da die Momente der Geschwindigkeitsverteilungen typischerweise über Zeiträume von wenigen Stunden korreliert sind. Einen alternativen Schätzwert für die Größenordnung der Varianz 4.7 liefert die über solche Zeiträume beobachtete Fluktuation der Teilchendichte der Protonen

$$
\left\langle f_{i}^{2}\right\rangle-\left\langle f_{i}\right\rangle^{2} \approx \frac{\left\langle\delta n^{2}\right\rangle}{\langle n\rangle^{2}}\left\langle f_{i}\right\rangle^{2} \approx \frac{\left\langle\delta n^{2}\right\rangle}{\langle n\rangle^{2}} f_{i}^{2},
$$

wobei typischerweise $\left\langle\delta n^{2}\right\rangle /\langle n\rangle^{2} \approx 5 \times 10^{-3}$ gilt. Im Vergleich mit der Gleichung 4.15 kommt dieser Wert der Varianz bei einer Zählrate von $Z \approx 200$ gleich, was der Größenordnung der Zählraten im Maximum der gemessenen Geschwindigkeitsverteilungen entspricht. Die Näherung 4.13 des asymptotischen MISE nimmt mit der Abschätzung 4.15, 
welche ich für die Auswertung der Meßdaten verwendet habe, die Form

$$
A_{\mathrm{MISE}}=\frac{V_{N}^{2}}{(4 \pi)^{3 / 2} h^{3}}\left(\sum_{i=1}^{N} \frac{f_{i}^{2}}{Z_{i}}+\frac{1}{4} \sum_{i, j=1}^{N} f_{i} f_{j} P\left(\frac{\left|\vec{v}_{i}-\vec{v}_{j}\right|}{2 h}\right) e^{-\left|\vec{v}_{i}-\vec{v}_{j}\right|^{2} / 4 h^{2}}\right)
$$

an. Der optimale Glättungsparameter 4.12 schreibt sich als

$$
\frac{h^{*}(h)}{h}=\left(\frac{1}{3 \sum_{i=1}^{N} f_{i}^{2} / Z_{i}} \sum_{i, j=1}^{N} f_{i} f_{j} P\left(\frac{\left|\vec{v}_{i}-\vec{v}_{j}\right|}{2 h}\right) e^{-\left|\vec{v}_{i}-\vec{v}_{j}\right|^{2} / 4 h^{2}}\right)^{-1 / 7} .
$$

Die Abbildung 4.2a zeigt den Verlauf des optimalen Glättungsparameters $h^{*}$ aus der Gleichung 4.19 in Abhängigkeit eines ersten Glättungsparameters $h$ für die Meßwerte der in der Abbildung 4.1 dargestellten Geschwindigkeitsverteilung. Wenn die durch eine Iteration der Beziehung 4.19 gewonnene Folge von Glättungsparametern konvergiert, ist ihr Grenzwert ein Fixpunkt $h^{*}(h)=h$. In der Abbildung ist zu erkennen, daß ein solcher Fixpunkt für dieses Beispiel nicht existieren kann, da das Verhältnis stets $h^{*}(h) / h<1$ erfüllt. Diese Eigenschaft gilt für alle in dieser Arbeit ausgewählten Datensätze. Die Bestimmung eines optimalen Glättungsparameters ist durch eine Iteration der Gleichung 4.19 deshalb nicht möglich. Nur unter der Annahme von Varianzen 4.7, die ungefähr um den Faktor $2^{7}=128$ größerer sind als die Abschätzungen 4.15 und 4.17 höbe sich die Kurve $h^{*}(h)$ soweit an, daß sich zwei Fixpunkten ergäben, von denen der größere den optimalen Glättungsparameter definierte.

In der Abbildung 4.2b ist der asymptotische MISE in der Form 4.18 als Funktion des Glättungsparameters für das Beispiel der Abbildung 4.1 aufgetragen. Er fällt sowohl für große als auch kleine Glättungsparameter wie $h^{-3}$ ab und besitzt deswegen nur ein lokales Minimum. Ich betrachte den durch dieses Minimum definierten Glättungsparameter als optimal. Seine Existenz ist allerdings nicht für jeden Datensatz gewährleistet. Die Abbildung 4.3a zeigt den asymptotischen MISE als Funktion des Glättungsparameters für eine solche gemessene Geschwindigkeitsverteilung der Protonen. Von den insgesamt 14142 fehlerfreien Datensätzen (s. Abschnitt 3.2) ließen nur 6103 die Bestimmung eines optimalen Glättungsparameters durch die Existenz des lokalen Minimums des asymptotischen MISE zu. Um auch die verbleibende größere Anzahl von Daten auszuwerten, betrachte ich in diesen Fällen die Stelle des Maximums des Verhältnisses $h^{*}(h) / h$ als optimal. Dieses Maximum existiert in jedem Fall und kommt einem gewünschten Fixpunkt des Zusammenhangs 4.19 am nächsten. Besitzt auch der asymptotische MISE ein lokales Minimum, muß der über das Verhältnis $h^{*}(h) / h$ definierte Glättungsparameter kleiner als die Stelle dieses Minimums sein. Da ich im Zweifel die glattere Modellverteilung als geeigneter erachte, möchte ich den durch das Maximum von $h^{*}(h) / h$ definierten Glättungsparameter nicht auf alle Meßdaten anwenden. In der Abbildung 4.3 sind die beiden alternativen optimalen Glättungsparameter als Streudiagramm für die Datensätze des 14. und 16. Aprils 1976 dargestellt. Zwischen ihnen besteht näherungsweise eine lineare Beziehung. Um zu vermeiden, daß eine Teilmenge der ausgewerteten Geschwindigkeitsverteilungen einen systematisch kleineren Glättungsparameter besitzt, habe ich aus den Datensätzen, die die Bestimmung beider optimalen Glättungsparameter erlauben, für jede der ausgewählten schnellen Sonnenwindströmungen (s. Tabelle 3.1) durch lineare Regression jeweils einen Zusammenhang zwischen beiden Glättungsparametern bestimmt. 
(a)

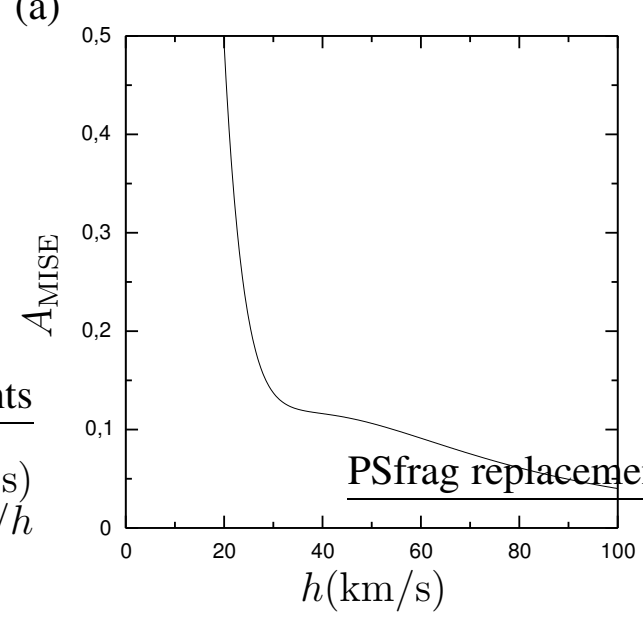

(b)

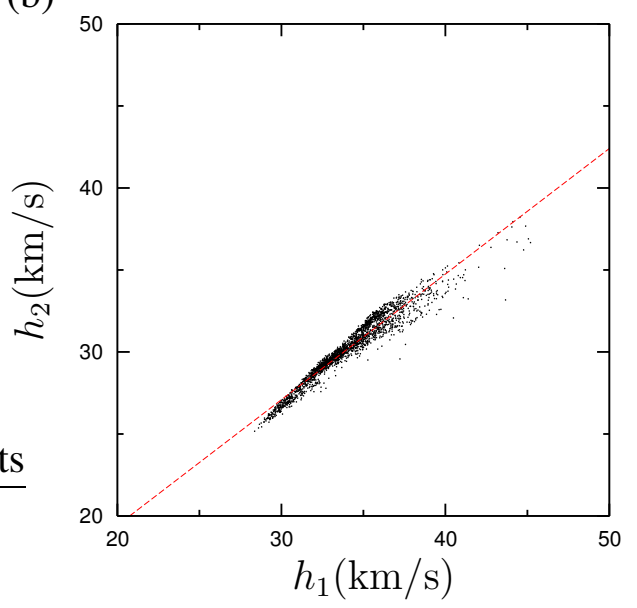

Abbildung 4.3: (a) Asymptotische Form 4.18 des MISE in Abhängigkeit von dem Glättungsparameter für die Werte der auf Helios 2 am 19. Februar 1976 um 0h/13m/30s gemessenen Geschwindigkeitsverteilung (s. Abbildungen 4.4a und b). (b) Empirischer Zusammenhang zwischen den über den asymptotischen MISE 4.18 und das Maximum des Verhältnisses $h^{*}(h) / h$ definierten optimalen Glättungsparametern $h_{1}$ bzw. $h_{2}$ für die Meßdaten des Instrumentes 1a auf Helios 2 vom 14. und 16. April 1976.

Dieser erlaubt es, den über das Maximum des Verhältnis $h^{*}(h) / h$ definierten optimalen Glättungsparameter anzugleichen.

Die Koeffizienten in der Entwicklung 4.1 sind zur Berechnung des Glättungsparameters durch $F_{i}=V_{N} f_{i}$ definiert. Der von der Anzahl der Meßwerte abhängige Faktor $V_{N}$ entfällt in den Gleichungen 4.18 und 4.19 und muß zu diesem Zweck nicht angegeben werden. Die Gleichung 4.8 eignet sich für eine endliche Anzahl von Messungen auch nicht für seine Berechnung, da das Gebiet $\mathcal{V}$ nicht eindeutig ist. Deshalb bestimme ich die Koeffizienten $F_{i}$, nachdem der optimale Glättungsparameter feststeht, durch die Minimierung der Summe der Fehlerquadrate

$$
\sum_{i=1}^{N}\left|f_{i}-f_{h}\left(\vec{v}_{i}\right)\right|^{2}
$$

unter der Nebenbedingung, daß $F_{i} \geq 0, i=1 \ldots, N$, gelten muß. Die Abbildung 4.4c zeigt das Resultat dieses Vorgehens für die Meßwerte der in der Abbildung 4.1 dargestellten Geschwindigkeitsverteilung. Es ist eine gute Übereinstimmung festzustellen. In der Abbildung 4.4b ist abschließend das Resultat der Auswertung der Meßwerte einer Geschwindigkeitsverteilung zu sehen, deren optimaler Glättungsparameter nicht über den asymptotischen MISE 4.18 bestimmbar ist und bereits angesprochen wurde (s. Abbbildung 4.3a). Der verwendete Glättungsparameter wurde über das Maximum $h^{*}(h) / h$ definiert und, wie oben beschrieben wird, reskaliert. Auch in diesem Fall ist eine gute Übereinstimmung feststellbar. Die bestimmte Entwicklung nach Kernfunktionen reicht über die in der Abbildung 4.4a erkennbaren Grenzen des Kanalgitters hinaus, so daß die Extrapolation durch diese Darstellung sichtbar wird.

Aus den Meßwerten der Geschwindigkeitsverteilungen der $\alpha$-Teilchen (s. Abschnitt 3.1.2) 
(a)

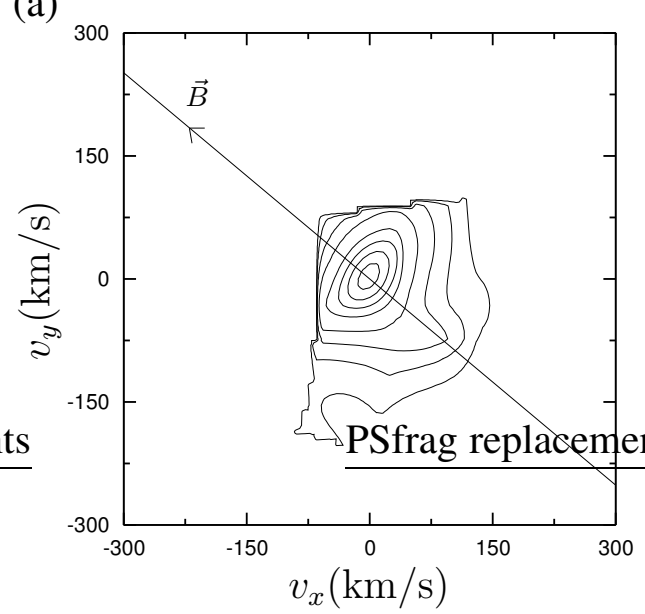

(b)

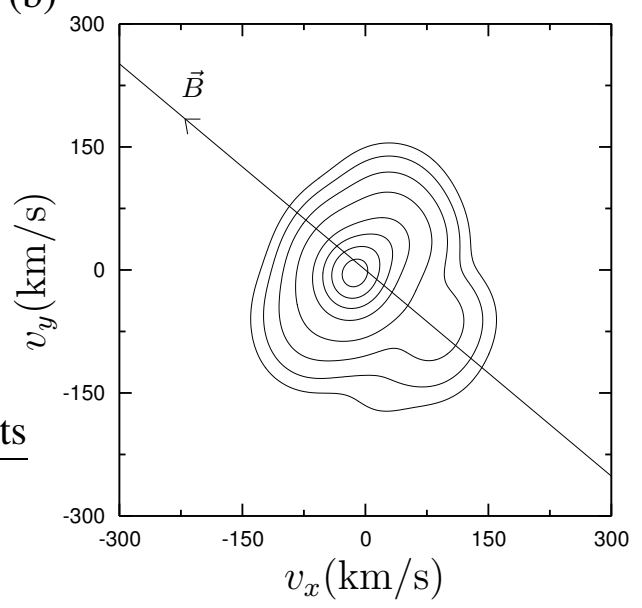

(c)

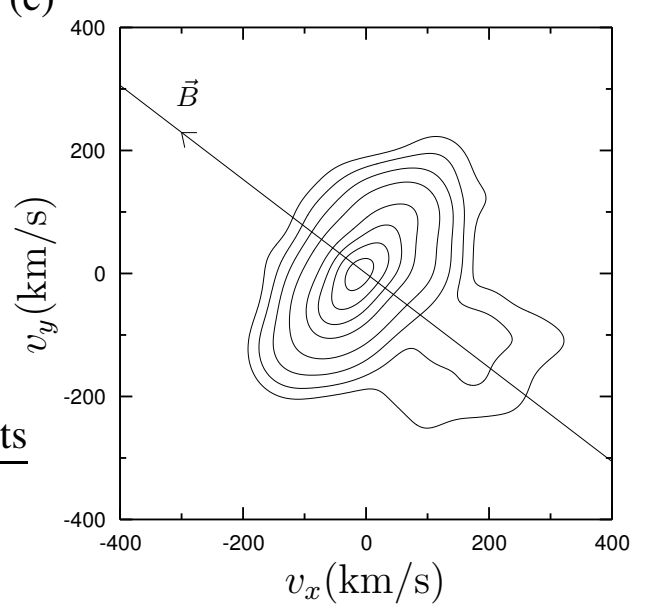

Abbildung 4.4: (a) Höhenlinien der polynomialen Interpolation der auf Helios 2 am 19. Februar 1976 um 0h/13m/30s gemessenen Geschwindigkeitsverteilung. (b) Höhenlinien derselben Verteilung in der Darstellung 4.1. (c) Höhenlinien der in der Abbildung 4.1 gezeigten Geschwindigkeitsverteilung in der Darstellung 4.1. Die Höhenlinien aller Teilabbildungen wurden in der aus der jeweiligen mittleren Geschwindigkeit und dem jeweiligen magnetischen Feld definierten Ebene berechnet. Die Abszisse zeigt in die Richtung der mittleren Geschwindigkeit und der Ursprung stimmt mit ihr überein. Die Höhenlinien entsprechen jeweils den in der Abbildung 4.1 verwendeten Anteilen der Maxima der Verteilungen.

können ebenso wie aus den Meßwerten der Verteilungen der Protonen auf die beschriebene Weise Modellverteilungen gewonnen werden. Die Zählraten der $\alpha$-Teilchen sind aber wegen ihrer geringen Teilchendichten klein. Deshalb ergeben sich im Vergleich mit den Protonen deutlich größere Glättungsparameter. Das Instrument ist somit nicht in der Lage, die Gestalt der Verteilungen der $\alpha$-Teilchen aufzulösen. Deshalb ersetze ich ihre in der Form 4.1 bestimmten Modellverteilungen in allen Berechnungen dieser Arbeit durch die äquivalenten Maxwell-Verteilungen. 



\section{Numerische Methode zur Lösung der kinetischen Dispersionsbeziehung}

Die im Abschnitt 2.2 diskutierte Dispersionsbeziehung kinetischer Plasmawellen kann nicht unmittelbar mit Standardmethoden zur Bestimmung von Nullstellen nicht-linearer Funktionen wie der Newton-Raphson-Iteration gelöst werden. Zwar existieren unter diesen numerischen Algorithmen solche, die global konvergent sind, d.h. die für jeden vorgegebenen Startwert eine Lösung finden. Allerdings besitzt die Dispersionsbeziehung für jeden Wellenvektor unendlich viele Lösungen. Ein Anwender ist jeder Kontrolle darüber beraubt, welche dieser Nullstellen er gefunden hat, wenn es ihm nicht möglich ist, einen Startwert vorzugeben, der innerhalb des Konvergenzradius des Verfahrens um die gewünschte Lösung liegt. Aus diesem Grund habe ich in dieser Arbeit ein zweiteiliges Verfahren umgesetzt. In einem ersten Schritt ermöglicht es, alle Frequenzen innerhalb eines vorgegebenen Ausschnittes der komplexen Ebene zu bestimmen, die die Dispersionsbeziehung bei einem festem Wellenvektor lösen. In einem zweiten Schritt wird die Abhängigkeit solcher Lösungen vom Wellenvektor berechnet. Auf diese Weise können Dispersionszweige stetig verfolgt werden. Die für den ersten Schritt des Verfahrens entscheidende Eigenschaft der Dispersionsbeziehung mit den in dieser Arbeit verwendeten Formen von Geschwindigkeitsverteilungen ist ihre analytische, d.h. holomorphe Abhängigkeit von der Frequenz (s. Abschnitt 2.2 und Anhang D). Den ersten Schritt des Verfahrens führe ich bei einem zum magnetischen Feld parallelen Wellenvektor durch, da die Dispersionsbeziehung für sich parallel zum magnetischen Feld ausbreitende Wellenmoden eine besonders einfache Form annimmt (s. Abschnitt 2.2.1). Alle Polarisationen sind bekannt und es müssen die Nullstellen der analytischen Dispersionsfunktionen $D_{ \pm}$und $D_{\|}$ für Zyklotron- und elektrostatische Wellenmoden (s. Gleichungen 2.17, 2.18, D.20, D.21) bestimmt werden. Im Abschnitt 5.1 beschreibe ich das von mir zu diesem Zweck verwendete Verfahren, das die Bestimmung der komplexen Nullstellen einer beliebigen holomorphen Funktion erlaubt. Im Abschnitt 5.2 wird erklärt, auf welche Weise sich der Dispersionszweig verfolgen läßt, der mit einer einzelnen Lösung der Dispersionsbeziehung verbunden ist. Im abschließenden Abschnitt 5.3 wird die Bestimmung der Abhängigkeit der Mode maximaler Anwachsrate eines Dispersionszweiges von Parametern, wie z.B. der Temperatur einer Teilchensorte, von denen die Geschwindigkeitsverteilungen und somit die Dispersionsbeziehung abhängen, diskutiert. 


\subsection{Bestimmung der Lösungen der Dispersionsbeziehung bei fixiertem Wellenvektor}

Die Anzahl der Nullstellen einer in einem Gebiet $G$ der komplexen Ebene analytischen Funktion $D$ kann mit Hilfe des Residuensatzes der Funktionentheorie bestimmt werden. Mit $D$ ist auch die Funktion $D^{\prime} / D$ bis auf die Nullstellen von $D$ in dem Gebiet G analytisch. Die Nullstellen sind ihre Pole, und die Ordnung eines Pols bzw. die Vielfachheit einer Nullstelle $\omega_{0} \in G$ stimmen mit dem Residuum

$$
\operatorname{Res}\left(\omega_{0}, \frac{D^{\prime}}{D}\right)=o\left(\omega_{0}, D\right)
$$

überein. Deshalb gilt

$$
\frac{1}{2 \pi i} \int_{\partial G} d \omega \frac{D^{\prime}(\omega)}{D(\omega)}=\sum_{\substack{f\left(\omega_{0}\right)=0 \\ \omega_{0} \in G}} o\left(\omega_{0}, f\right)
$$

wobei $\partial G$ die Kurve bezeichne, die das Gebiet $G$ umrandet. Durch die Kenntnis einer analytischen Funktion und ihrer Ableitung auf dem Rand eines Gebietes ist es also möglich, ihre Nullstellen in diesem Gebiete mit ihrer Vielfachheit zu zählen. Besitzt die Funktion $D$ nur einfache Nullstellen, so lassen sie sich durch die schrittweise Zweiteilung des Gebietes isolieren. Die Teilung wird solange fortgeführt, bis jedes Teilgebiet $G^{\prime}$ nur eine einzige Nullstelle $\omega_{0}$ enthält. Der Residuensatz erlaubt es auch, sie zu berechnen

$$
\int_{\partial G^{\prime}} d \omega \frac{\omega D^{\prime}(\omega)}{D(\omega)}=\omega_{0}, \quad \omega_{0} \in G^{\prime} \subset G .
$$

Zur numerischen Bestimmung der Randintegrale wird eine Gaußsche Quadratur verwendet und als Ausgangsgebiet stets ein Rechteck in der komplexen Ebene gewählt. Die Bisektion geschieht durch Halbierung einer der Seiten des Rechtecks. Die Genauigkeit des Resultates der Integration 5.3 wird abschließend durch eine Newton-Raphson-Iteration verbessert, deren Konvergenz nun garantiert ist. Auf dieser Weise werden alle Nullstellen der Funktion $D$ in dem Ausgangsgebiet $G$ bestimmt.

Einfache und dennoch nicht triviale Beispiele, an denen sich dieses Verfahren bewährt, sind die komplexe Fehlerfunktion erf (s. Abramowitz und Stegun 1965, S. 297ff.) und die Plasmadispersionsfunktion $Z$, die sich aus der Fehlerfunktion ableitet (s. Gleichung 2.29). Beide Funktionen sind in der ganzen komplexen Ebene analytisch und besitzen unendlich viele einfache Nullstellen. Die Abbildung 5.1 zeigt die mit der in diesem Abschnitt beschriebenen Methode bestimmten Nullstellen beider Funktionen im durch $0 \leq \operatorname{Re} z \leq 10$ und $-10 \leq \operatorname{Im} z \leq 0$ definierten Quadranten der komplexen Ebene. Weitere Nullstellen der Funktionen ergeben sich aus den bekannten Eigenschaften $\operatorname{erf}(z)=\operatorname{erf}\left(z^{*}\right)^{*}=$ $-\operatorname{erf}(-z)$ und $Z(z)=-Z\left(-z^{*}\right)^{*}$. Die ersten zehn Nullstellen der Fehlerfunktion sind z.B. in der Sammlung von Abramowitz und Stegun (1965) tabelliert und stimmen mit denen der Abbildung 5.1überein. 


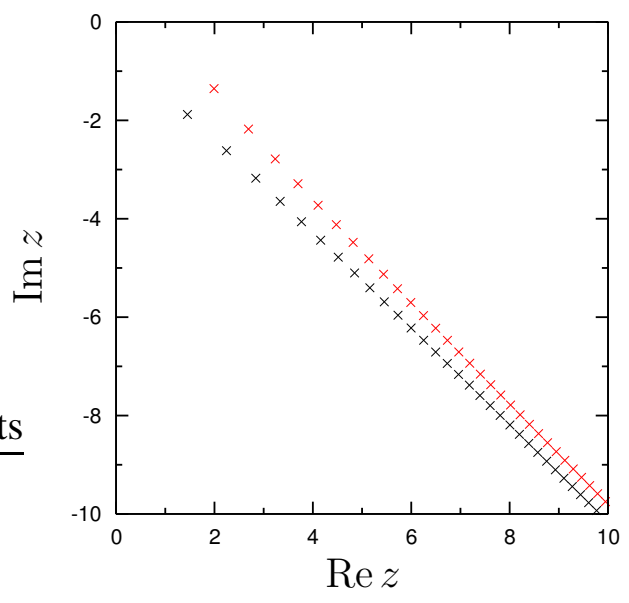

Abbildung 5.1: Alle Nullstellen der komplexen Fehlerfunktion erf (schwarz) und der Plasmadispersionsfunktion $Z$ (rot) im durch $0 \leq \operatorname{Re} z \leq 10$ und $-10 \leq \operatorname{Im} z \leq 0$ definierten Bereich der komplexen Ebene. Die erste und die letzte der jeweils 31 Nullstellen lauten $z_{1}=1.450616-1.880943 i, z_{31}=9.767493-9.930268 i$ für die Fehlerfunktion und $z_{1}=1.991467-1.354810 i, z_{31}=9.947978-9.750015 i$ für die Plasmadispersionsfunktion.

Die Dispersionsfunktionen $D_{\|, \pm}$kinetischer Plasmawellen setzen sich für die in dieser Arbeit verwendeten Geschwindigkeitsverteilungen aus der Plasmadispersionsfunktion zusammen. Außer bei unter Umständen vorhandenen isolierten Wellenzahlen $k_{\|}$besitzen auch sie nur einfache Nullstellen.

\subsection{Bestimmung der Abhängigkeit der Lösungen der Di- spersionbeziehung vom Wellenvektor}

Zur Bestimmung der Abhängigkeit einer Lösung der Dispersionsbeziehung vom Wellenvektor wende ich ein Homotopie-Verfahren an. Mit einem solchen Verfahren lassen sich generell die Nullstellen einer Funktion $H_{1}$ aus den bekannten Nullstellen einer anderen Funktion $H_{0}$ berechnen. Dabei ist die Homotopie ein Übergang $H(\lambda, z)$ zwischen den Funktionen mit einem Homotopieparameter $0 \leq \lambda \leq 1$, d.h. es gilt

$$
H(\lambda=0, z)=H_{0}(z), \quad H(\lambda=1, z)=H_{1}(z) .
$$

$\mathrm{Zu}$ jeder Nullstelle $z_{0}$ von $H_{0}$ ist die Kurve $z(\lambda)$ mit $H(\lambda, z(\lambda))=0$ und $z(0)=z_{0}$ gesucht. Diese Kurve erfüllt unter Annahme der hinreichenden Differenzierbarkeit der Homotopie die Differentialgleichung

$$
\frac{d H(\lambda, z(\lambda))}{d \lambda}=\sum_{\nu} \frac{\partial H(\lambda, z(\lambda))}{\partial z_{\nu}} z_{\nu}^{\prime}(\lambda)+\frac{\partial H(\lambda, z(\lambda))}{\partial \lambda}=0,
$$

welche sich, solange die Jakobi-Matrix der Homotopie invertierbar bleibt, integrieren läßt. Der von mir verwendete und darauf basierende Algorithmus wurde von Watson et al. (1987) beschrieben und implementiert. 
In dieser Arbeit muß das Verfahren auf die Berechnung von Kurven $z(p)$ von Nullstellen einer Funktion $F(z, p)$ in Abhängigkeit von einem Parameter $p$ angewendet werden, wobei Anfangslösungen $z\left(p_{0}\right)$ bekannt sind und die Funktion $F$, die Variable $z$ und der Parameter $p$ mehrere reellwertige Komponenten besitzen. Es bieten sich die Homotopien

$$
H(\lambda, z)=F\left(z, \lambda p+(1-\lambda) p_{0}\right)
$$

oder

$$
H(\lambda, z)=\lambda F(z, p)+(1-\lambda) F\left(z, p_{0}\right) .
$$

an. Der Parameter $p$ umfaßt die Komponenten des Wellenvektors $p=\left(k_{\|}, k_{\perp}\right)$. Er könnte aber prinzipiell auch die Parameter der verwendeten Geschwindigkeitsverteilungen der Teilchensorten bezeichnen. Durch die Definitionen

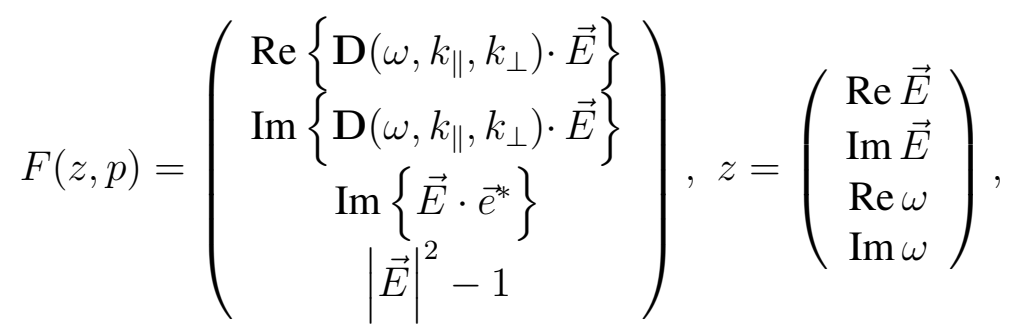

der Funktion $F$ und der Variablen $z$ entsprechen die Nullstellen von $F$ den Lösungen der Dispersionsbeziehung

$$
\mathbf{D}\left(\omega\left(k_{\|}, k_{\perp}\right), k_{\|}, k_{\perp}\right) \cdot \vec{E}\left(k_{\|}, k_{\perp}\right)=0 .
$$

Die zusätzlichen Bedingungen

$$
\operatorname{Im}\left\{\vec{E} \cdot \vec{e}^{*}\right\}=0, \quad|\vec{E}|^{2}=1
$$

dienen dazu, die Entartung $\vec{E} \rightarrow c \vec{E}, c \in \mathbb{C}$, aufzuheben. Der konstante Vektor $\vec{e}$ wird für die Integration der Differentialgleichung 5.5 auf die Anfangspolarisation gesetzt. Durch die Homotopien 5.6 und 5.7 können die Punkte eines regulären Gitters von Wellenvektoren verbunden werden. Das Gitter enthält einen Anfangspunkt bei $k_{\perp}=0$, bei dem Lösungen der Dispersionbeziehung 5.9 auf die im Abschnitt 5.1 beschriebene Weise als Nullstellen von $D_{ \pm}$oder $D_{\|}$bestimmt werden. Ohne Einschränkung der Allgemeinheit ist der Polarisationsvektor dort $\vec{E}=\left(\vec{e}_{1} \pm i \vec{e}_{2}\right) / \sqrt{2}$ für Nullstellen von $D_{ \pm}$und $\vec{E}=\vec{B} / B$ für Nullstellen von $D_{\|}$, wobei $\vec{B}$ das magnetische Feld und $\vec{e}_{1}$, $\vec{e}_{2}$ beliebige zu $\vec{B} / B$ orthonormale Vektoren bezeichnen (s. Abschnitt 2.2).

\subsection{Bestimmung der Parameterabhängigkeit von Moden maximaler Anwachsrate}

Die Eigenschaft maximaler Anwachsrate drückt sich durch die Bedingungen

$$
\frac{\partial \operatorname{Im} \omega}{\partial k_{\|}}=\frac{\partial \operatorname{Im} \omega}{\partial k_{\perp}}=0
$$


aus, welche zusätzlich zu der Dispersionsbeziehung 5.9 erfüllt sein müssen. Durch die in der Gleichung 5.8 definierten Variablen $z$ und den Parameter $p$ lassen sich diese Bedingungen in der allgemeinen Form

$$
\sum_{\mu} C_{\mu} \frac{d z_{\mu}}{d p}
$$

mit Konstanten $C_{\mu}$ schreiben, wobei in diesem Fall mit Ausnahme des Index $\mu$ mit $z_{\mu}=$ $\operatorname{Im} \omega$ alle Konstanten $C_{\mu}$ verschwinden müssen. Ein Mode maximaler Anwachsrate stimmt also mit einer Nullstelle der erweiterten Funktion

$$
\widetilde{F}(\widetilde{z})=\left(\begin{array}{c}
F(z, p) \\
\frac{\partial F}{\partial z} \cdot \frac{d z}{d p}+\frac{\partial F}{\partial p} \\
C \cdot \frac{d z}{d p}
\end{array}\right)
$$

in der neuen Variablen $\widetilde{z}=(z, d z / d p, p)$ überein. Dabei müssen $z$ und $d z / d p$ als unabhängig aufgefaßt werden. Die Indizes in den Ableitungen $\partial F_{\mu} / \partial z_{\nu}$, etc. sind ausgelassen worden. Über die Dispersionsmatrix $\mathbf{D}$ hängen die Funktionen $F$ und $\widetilde{F}$ von den Parametern $q$ der verwendeten Geschwindigkeitsverteilungen der Teilchensorten des Plasmas ab. Die Bestimmung einer Nullstellenkurve $\widetilde{z}(q)$ von $\widetilde{F}$ kann mit dem Verfahren von Watson et al. (1987) und einer der Homotopien 5.6 und 5.7 bewältigt werden. Anfangslösungen $\widetilde{z}\left(q_{0}\right)$ werden aus dem bei $q_{0}$ berechneten Verlauf von Dispersionszweigen abgelesen. Die Berechnung findet auf einem Gitter mit hinreichender Auflösung statt. Die Frequenz, der Wellenvektor und der Polarisationsvektor der auf dem Gitter vorhandenen Mode maximaler Anwachsrate dienen als Startwerte für eine Newton-Raphson-Iteration bezüglich $\widetilde{F}$.

Stabilitätsgrenzen von Mikroinstabilitäten unter den Parametern $q$ sind durch einen konstanten Wert $\gamma_{0}$ der maximalen Anwachsrate festgelegt. Stellen auf dieser Grenze können als Nullstellen derjenigen Funktion erhalten werden, die sich durch die Erweiterung von $\widetilde{F}$ um die Komponente $\operatorname{Im} \omega-\gamma_{0}$ ergibt. Die Variable $\widetilde{z}$ muß um eine beliebige Komponente der Parameter $q$ erweitert werden. Die Abhängigkeit der Stabilitätsgrenzen von den verbleibenden Parametern in $q$ kann wiederum durch das Verfahren von Watson et al. (1987) berechnet werden.

Die in den Abbildungen 2.4a bis 2.4f gezeigten Parameterabhängigkeiten der maximalen Anwachsraten spezieller Mikroinstabilitäten und die in den Abbildungen 2.5a und 2.5b dargestellten Stabilitätsgrenzen dieser Mikroinstabilitäten sind auf die hier beschriebene Weise berechnet worden. Der Vergleich mit den Ergebnissen von Daughton und Gary (1998) zeigt eine Übereinstimmung. 



\section{Untersuchung von Mikroinstabilitäten im schnellen Sonnenwind}

In diesem Kapitel der Arbeit soll eine Untersuchung des schnellen Sonnenwindes auf Mikroinstabilitäten beschrieben werden (s. Abschnitt 1.2 und 1.3 der Einleitung). Aus der Beobachtung linearer Instabilitäten darf geschlußfolgert werden, daß die betreffenden Moden angeregt sind. Ihre Wechselwirkung mit den Teilchen des Sonnenwindes beeinflußt möglicherweise entscheidend deren Geschwindigkeitsverteilungen (Schwartz et al. 1981). Die marginale Stabilität der Moden stellt ggf. eine beobachtbare Zwangsbedingung für die Parameter der Verteilungen dar, wie z.B. der Relativgeschwindigkeit des Protonenstrahls im Fall einer durch ihn ausgelösten Instabilität (s. Abschnitt 2.2.3). Eine Abweichung von der marginalen Stabilität zeigt, daß die betreffenden Moden einer nichtlinearen Wechselwirkung ausgesetzt sind, die nicht vernachlässigt werden darf. Die Anwachsraten instabiler Moden wurden direkt aus gemessenen Geschwindigkeitsverteilungen berechnet. Die für diesen Zweck verwendeten Meßdaten des Plasmaexperimentes auf Helios 2 stammen aus dem solaren Minimum um das Jahr 1976 (s. Abschnitt 3.2). Es sind die Geschwindigkeitsverteilungen aller vom Plasmaexperiment gleichzeitig gemessenen Teilchenarten, d.h. der Protonen, Elektronen und $\alpha$-Teilchen, in die Untersuchung einbezogen worden. Die Geschwindigkeitsverteilungen der Elektronen und $\alpha$-Teilchen (s. Abschnitte 3.1.3 und 4.3) habe ich durch ihre äquivalenten Maxwell-Verteilungen berücksichtigt. Diese Vereinfachung gilt ebenso für die Untersuchung des Kapitels 7. Die Geschwindigkeitsverteilungen der Protonen werden in Abhängigkeit von der Ausbreitungsrichtung der Mode maximaler Anwachsrate einer Instabilität auf unterschiedliche Weise in die Untersuchung einbezogen. Für sich schräg ausbreitende Wellenmoden kann die Dispersionsmatrix 2.11 für die Darstellung 4.1 der Verteilungen der Protonen nicht berechnet werden. Deshalb vereinfache ich die Geschwindigkeitsverteilungen zu einer Überlagerung von zwei Bi-Maxwell-Verteilungen, welche einen Kern und einen Strahl modellieren sollen. Im Abschnitt 6.1 dieses Kapitels erkläre ich, wie sich ein zutreffendes Kern-Strahl-Modell aus der allgemeinen Darstellung 4.1 gewinnen läßt. Die Dispersionsbeziehung von sich parallel zum mittleren magnetischen Feld ausbreitenden Wellenmoden hängt nur von den reduzierten Geschwindigkeitsverteilungen nullter und erster Ordnung ab (s. Abschnitt 2.2.1). Die reduzierten Verteilungen der allgemeinen Darstellung 4.1 können bestimmt werden (s. Anhang C). Allerdings besitzt die Entwicklung 4.1 nach Gaußfunktionen im Perihel von Helios oft mehr als 100 Summanden. Zugleich umfaßt die ausgewählte Datenmenge ungefähr 10000 Datensätze. Um den zeitlichen Aufwand der 
Auswertung zu begrenzen, wird die Anzahl der Summanden in den reduzierten Verteilungen, die selber Entwicklungen nach Gaußfunktionen sind, geeignet verringert. Dieses Verfahren beschreibe ich im Abschnitt 6.2. Die Dispersionsbeziehung (s. Abschnitt 2.2.2 bzw. Anhang D) wurden auf die im Kapitel 5 beschriebene Weise gelöst. Die Ergebnisse der Untersuchung auf Instabilitäten mit Moden maximaler Anwachsrate, die sich schräg zum mittleren magnetischen Feld ausbreiten, beschreibe ich im Abschnitt 6.3. Dabei handelt sich ausschließlich um solche Instabilitäten, die vom Protonenstrahl verursacht werden können: die Alfvén I, II und III Instabilitäten sowie eine Instabilität der magnetosonischen Moden (s. Abschnitt 2.2.3). Im Abschnitt 6.4 schildere ich die entsprechenden Resultate für die Instabilitäten, deren Mode maximaler Anwachsrate sich parallel zum magnetischen Feld ausbreitet. Diese Instabilitäten können sowohl durch den Protonenstrahl als auch durch die Temperaturanisotropie des Kerns der Verteilung der Protonen ausgelöst werden und betreffen die magnetosonischen Moden bzw. die Alfvén-Moden (s. Abschnitt 2.2.3).

\subsection{Zerlegung der Geschwindigkeitsverteilungen der Pro- tonen in Kern und Strahl}

In der Einleitung zu diesem Kapitel ist darauf hingewiesen worden, daß es nötig ist, die im Kapitel 4 beschriebene Darstellung der Geschwindigkeitsverteilungen der Protonen zu vereinfachen. Die Dispersionsbeziehung von sich schräg zum mittleren magnetischen Feld ausbreitenden Moden läßt sich anderenfalls nicht bestimmen. Dafür zerlege ich die Darstellung 4.1 der Verteilungen in einen Kern und einen Strahl, welche ich durch BiMaxwell-Verteilungen modelliere. Diese Zerlegung ist auch dann möglich, wenn sich der Protonenstrahl nicht als Nebenmaximum in der Verteilung zeigt sondern nur eine Flanke des Kerns bildet. Sie findet innerhalb der reduzierten Verteilung nullter Ordnung (s. Gleichung 2.19) statt. Im Anhang $\mathrm{C}$ zeige ich, daß diese reduzierte Verteilung für die Darstellung 4.1 die Form

$$
F_{0}\left(v_{\|}\right)=\left(2 \pi h^{2}\right)^{-1 / 2} \sum_{i=1}^{N} F_{i} e^{-\left(v_{\|}-u_{i \|}\right)^{2} / 2 h^{2}}
$$

mit $u_{i \|}=\left(\vec{v}_{i}-\vec{u}\right) \cdot \vec{b}$ besitzt, wobei $F_{i}, \vec{v}_{i}$ und $h$ die Entwicklungskoeffizienten, die Positionen der Gaußfunktionen bzw. der Glättungsparameter in der Darstellung 4.1 sind. Der Richtungsvektor des mittleren magnetischen Feldes und die mittlere Geschwindigkeit der Verteilung werden durch $\vec{b}$ bzw. $\vec{u}$ bezeichnet. In der reduzierten Verteilung sind der Kern und der Strahl weiterhin erkennbar, da die Relativgeschwindigkeit des Strahls im Sonnenwind nahezu parallel zum mittleren magnetische Feld gerichtet ist. Ohne Einschränkung der Allgemeinheit steigt die Folge der Geschwindigkeiten $u_{i \|}$ monoton an. Durch die Wahl eines Index $i$ ergibt sich mit den Teilsummen über die Indizes $j$ mit $1 \leq j<i$ und $i \leq j \leq N$ eine Zerlegung der reduzierten Verteilung in einen langsamen und einen schnellen Anteil. Die Stelle $i$ ist nun so zu bestimmen, daß diese Anteile als Kern bzw. als Strahl der Verteilung bezeichnet werden dürfen. Es seien $F_{<}$und $F_{\geq}$die zu den genannten Teilsummen in Bezug auf die Momente äquivalenten Maxwell-Verteilungen. Ich 
(a)

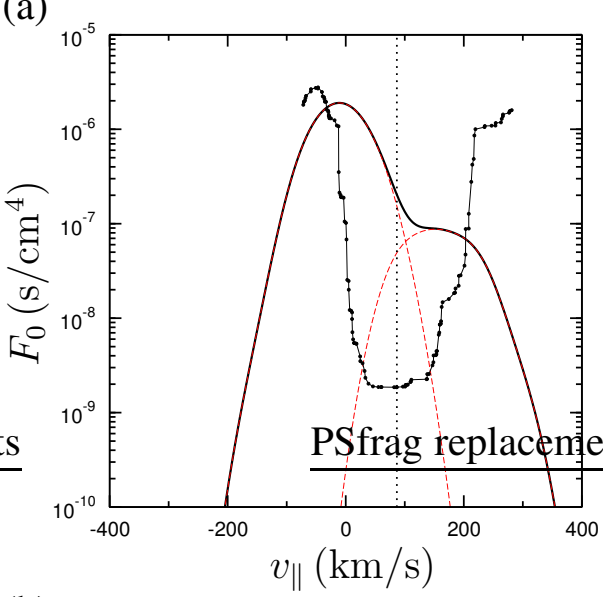

(b)

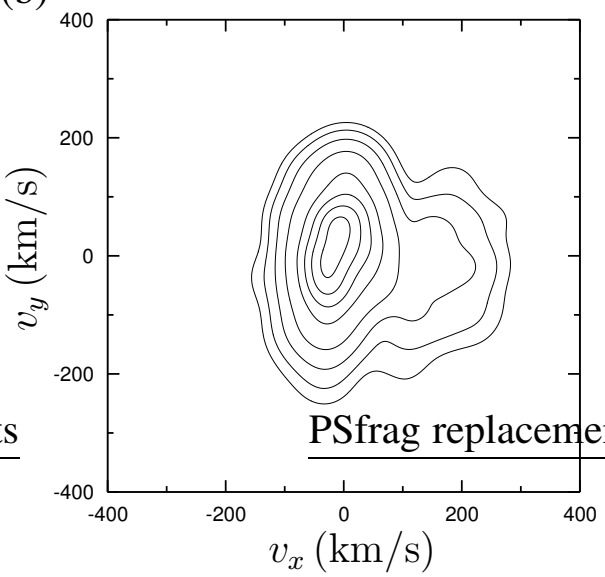

(c)

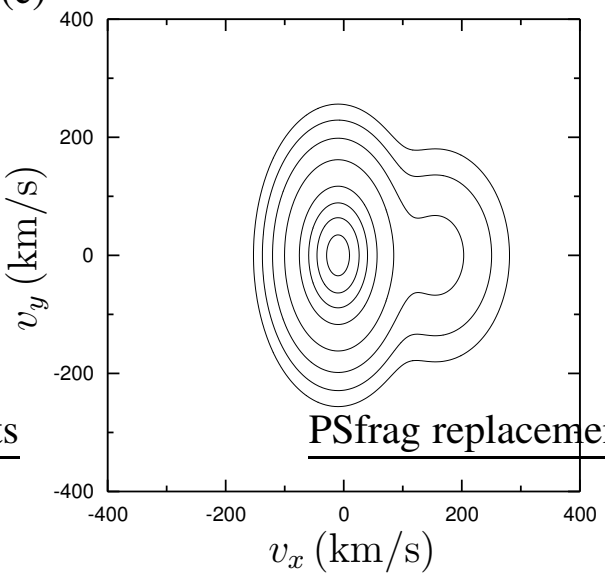

(d)

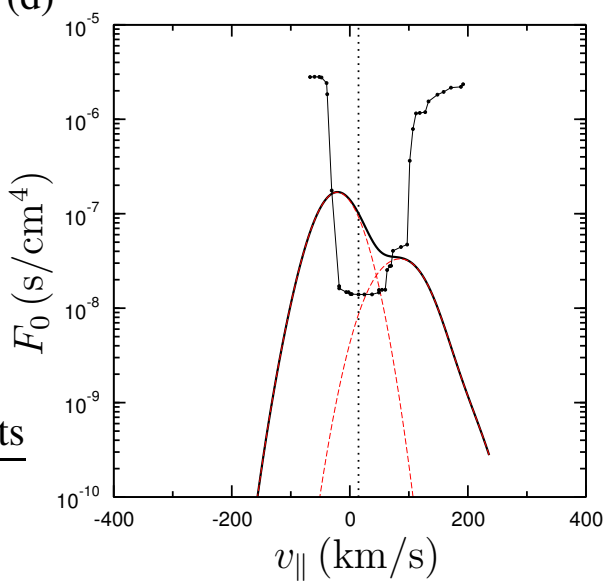

(e)

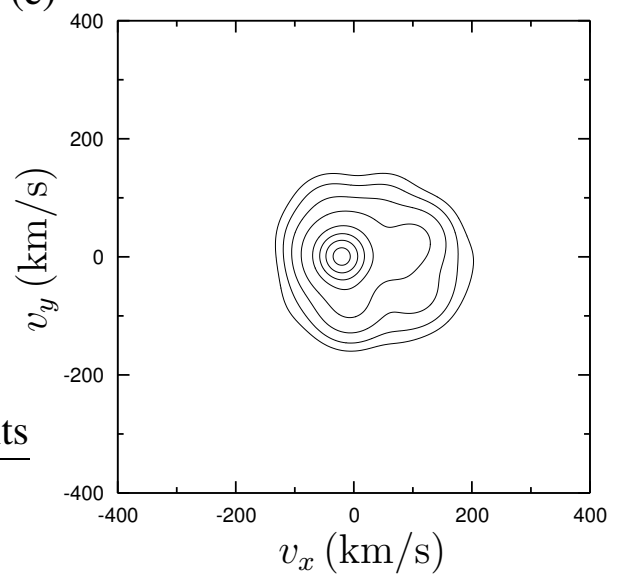

(f)

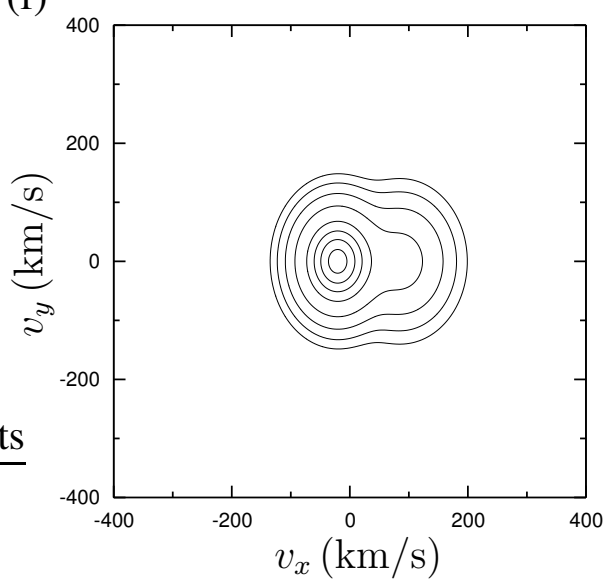

Abbildung 6.1: Trennung von zwei Geschwindigkeitsverteilungen in Kern und Strahl (links Helios 2, 14. April, 10h/37m/21s, 1976 und rechts Helios 2, 23. Januar, 21h/55m/7s, 1976). Die Abbildungen (a) und (d) zeigen die reduzierten Verteilungen nullter Ordnung $F_{0}$ sowie den Abstand von $F_{<}+F_{\geq}$(s. Text) zu $F_{0}$ als Funktion der Trennstelle. Eine Skala für diesen Abstand ist nicht angegeben. Die Abbildungen (b) und (e) sowie (c) und (f) zeigen Höhenlinien der Geschwindigkeitsverteilungen bzw. der Superpositionen aus BiMaxwell-Verteilungen für Kern und Strahl. Die Höhenlinien entsprechen den Anteilen 0.9, 0.7, 0.5, 0.3, 0.1, 0.03, 0.01 und 0.003 der Maxima der Verteilungen. Die Abszisse ist parallel zum magnetischen Feld und der Ursprung stimmt jeweils mit der mittleren Geschwindigkeit der Verteilung überein. 
wähle ich die Trennstelle $i$ so, daß der Abstand

$$
\int d v_{\|}\left|F_{0}\left(v_{\|}\right)-F_{<}\left(v_{\|}\right)-F_{\geq}\left(v_{\|}\right)\right|^{2}
$$

zwischen der Summe der äquivalenten Maxwell-Verteilungen und der reduzierten Verteilung minimal ist. Die Abbildungen 6.1a-6.1c und 6.1d-6.1f zeigen das Resultat der beschriebenen Vorgehensweise für zwei exemplarische im schnellen Sonnenwind gemessene Geschwindigkeitsverteilungen, die aus einer Entfernung von 0.30 AE bzw. 0.98 AE stammen. Der Anteil des Protonenstrahls an der Teilchendichte der Protonen ist im ersten Fall mit 0.06 klein, während er im anderen Fall mit 0.23 groß ist. Die Geschwindigkeiten $u_{i \|}$ aus der Entwicklung der reduzierten Verteilung nullter Ordnung sind in den Abbildungen 6.1a und 6.1d an den Positionen der schwarzen Punkte zu erkennen, die den Verlauf des zu minimierenden Abstandes 6.2 in Abhängigkeit von der Trennstelle $i$ markieren. Die dem Kern und dem Strahl entsprechenden Teilsummen innerhalb der reduzierten Verteilungen nullter Ordnung sind durch die roten gestrichelten Kurven dargestellt. Die reduzierte Verteilung erster Ordnung

$$
F_{1}\left(v_{\|}\right)=\left(2 \pi h^{2}\right)^{-1 / 2} \sum_{i=1}^{N} 2 h^{2} F_{i}\left(1+\frac{u_{i \perp}^{2}}{2 h^{2}}\right) e^{-\left(v_{\|}-u_{i \|}\right)^{2} / 2 h^{2}}
$$

wird an derselben Trennstelle $i$ zerlegt, die für die reduzierte Verteilung nullter Ordnung bestimmt wurde. Mit $u_{i \perp}$ wird der Betrag des Vektors $\vec{v}_{i}-\vec{u}$ senkrecht zum mittleren magnetischen Feld bezeichnet. Die Momente der Bi-Maxwell-Verteilungen, die den Kern bzw. den Strahl modellieren sollen, können aus den reduzierten Verteilungen nullter und erster Ordnung von Kern und Strahl, welche sich durch die beschriebene Zerlegung ergeben, bestimmt werden. Die Teilchendichte $n$, die mittlere und die thermische Geschwindigkeit parallel zum magnetischen Feld $u_{\|}$bzw. $v_{T \|}$ von Kern und Strahl ergeben sich jeweils aus der nullten Ordnung

$$
\begin{gathered}
n=\int d v_{\|} F_{0}\left(v_{\|}\right), \\
n u_{\|}=\int d v_{\|} v_{\|} F_{0}\left(v_{\|}\right),
\end{gathered}
$$

und

$$
n v_{T \|}^{2}=\int d v_{\|}\left(v_{\|}-u_{\|}\right)^{2} F_{0}\left(v_{\|}\right)
$$

Die thermischen Geschwindigkeiten $v_{T \perp}$ von Kern und Strahl senkrecht zum mittleren magnetischen Feld ergeben sich jeweils aus der ersten Ordnung

$$
2 n v_{T \perp}^{2}=\int d v_{\|} F_{1}\left(v_{\|}\right)
$$

Die mittlere Geschwindigkeit $u_{\|}$bezieht sich auf den Ursprung $\vec{u}$, d.h. die Bi-MaxwellVerteilungen von Kern und Strahl müssen jeweils bei der Geschwindigkeit $\vec{u}+u_{\|} \vec{b}$ platziert werden. Das Resultat dieser Bestimmung äquivalenter Bi-Maxwell-Verteilungen zeigen die Abbildungen 6.1c und 6.1f. 


\subsection{Vereinfachung der reduzierten Verteilungen der Pro- tonen}

Die Anzahl der Summanden in der Entwicklung 4.1 einer Geschwindigkeitsverteilung der Protonen nach Gaußfunktionen variiert zwischen den Größenordnungen 29 im Aphel von Helios bei kleinen Teilchendichten und 214 im Perihel von Helios bei großen Teilchendichten. Für die größere Anzahl nimmt die erforderliche Rechenzeit, um die Dispersionsbeziehung zu lösen, pro Geschwindigkeitsverteilung einen Umfang an, der es praktisch unmöglich macht, die insgesamt 10804 Verteilungen (s. Abschnitt 3.2) auszuwerten. Aus diesem Grund fasse ich die zu den reduzierten Verteilungen nullter und erster Ordnung beitragenden Summanden (s. Gleichung C.16 bzw. C.17) in Gruppen zusammen und reduziere damit ihre Anzahl. Das Zusammenfassen mehrerer Summanden zu einem einzigen Term der gleichen Gestalt soll zu einer möglichst guten Approximtion der ursprünglichen reduzierten Verteilungen führen. Dafür wende ich die im Abschnitt 6.1 beschriebene Methode an. Die Summanden einer reduzierten Verteilungen werden in zwei Gruppen aufgeteilt, so daß die Überlagerung der zu den Teilsummen äquivalenten Maxwell-Verteilungen den geringsten Abstand zur Ausgangsverteilung besitzt. Jede der beiden Gruppen von Summanden läßt sich erneut zweiteilen. Auf diese Weise wird die Menge aller Summanden der reduzierten Verteilung nach $n$ Schritten in maximal $2^{n}$ Teilmengen aufgeteilt. Die Summe der zu den Teilsummen äquivalenten MaxwellVerteilungen besitzt dieselbe mathematische Gestalt wie die Ausgangsverteilung und soll als Approximation verwendet werden. Um einen akzeptablen Zeitaufwand zu erzielen, habe ich in jedem Einzelfall die reduzierten Verteilungen nullter und erster Ordnung unabhängig voneinander durch $n=4$ Zerlegungsschritte auf die Größe von maximal 16 Summanden verringert. Die Abbildungen 6.2a und 6.2b zeigen zum Vergleich die reduzierten Verteilungen nullter bzw. erster Ordnung vor und nach der Verringerung der Anzahl der Summanden für ein Beispiel aus dem Perihel von Helios 2. In den Abbildung 6.2c und 6.2d sind ebenfalls zum Vergleich die Dispersionszweige im Bereich der Zyklotronfrequenzen der Ionen (vgl. Abbildung 2.1b) und die Anwachsraten der bestehenden Instabilitäten vor und nach der Verringerung dargestellt. Die Instabilität bei positiven Wellenzahlen betrifft die auswärts laufenden Alfvén-Moden. Bei negativen Wellenzahlen sind die Moden des in der Abbildung 2.1b gepunktet gezeichneten Zyklotron-Zweiges betroffen. Er ist auch in der Abbildung 6.2c zu sehen. Beide Instabilitäten werden durch die Temperaturanisotropie des Kerns der Verteilung der Protonen ausgelöst. Die vorhandenen Unterschiede können als gering bezeichnet werden. Ich habe ein Beispiel aus dem Perihel gewählt, da die Anzahl der Summanden und somit der Einfluß der beschriebenen Verringerung ihrer Anzahl dort am größten ist. Im gezeigten Fall beträgt die Anzahl der Summanden vor der Verringerung 165, und danach sind es 16 und 14 Summanden in den reduzierten Verteilungen nullter bzw. erster Ordnung. Die Abbildung 6.3a zeigt den Umfang, den die Verringerung der Summandenanzahl in verschiedenen Entfernungen zur Sonne annimmt. Im Perihel entfallen im Mittel mehr als $90 \%$ aller Summanden aus den Darstellungen der reduzieren Verteilungen. Im Aphel sind es noch 50\%. Dennoch variiert der verursachte Fehler, ausgedrückt durch den von der Norm $\|F\|^{2}=\int d v_{\|}\left|F\left(v_{\|}\right)\right|^{2}$ definierten relativen Abstand der verringerten reduzierten Verteilung zur Ausgangsverteilung, nur schwach mit dem Abstand und bleibt unterhalb von 1\%. Diese Beobachtungen spre- 
(a)

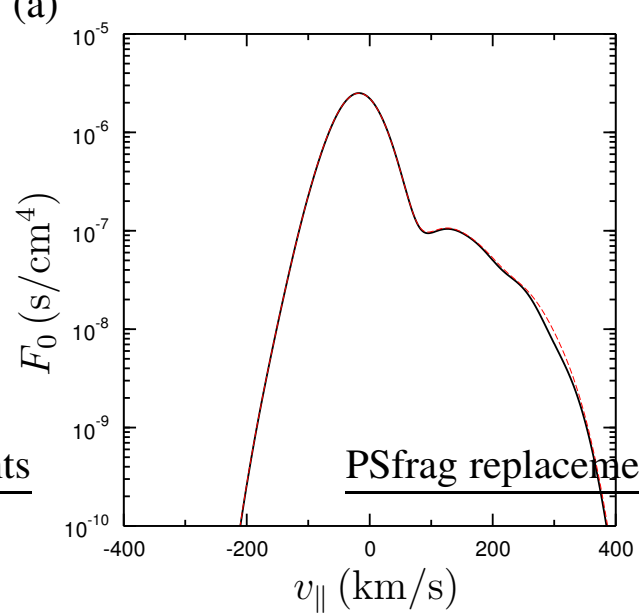

(c)

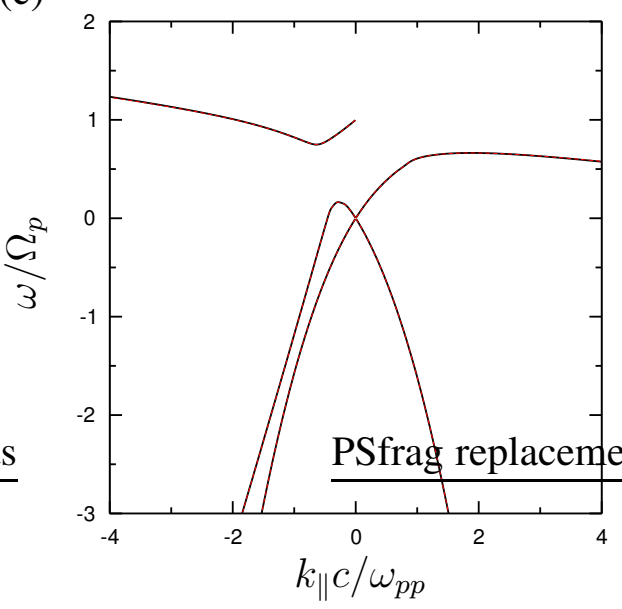

(b)

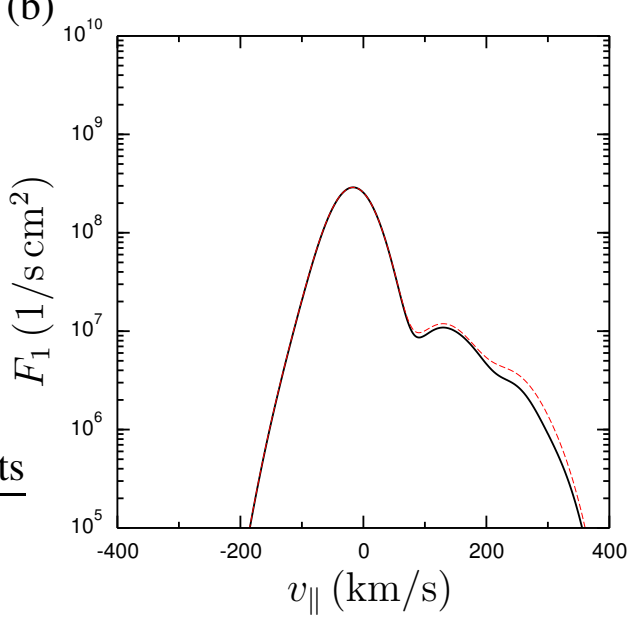

(d)

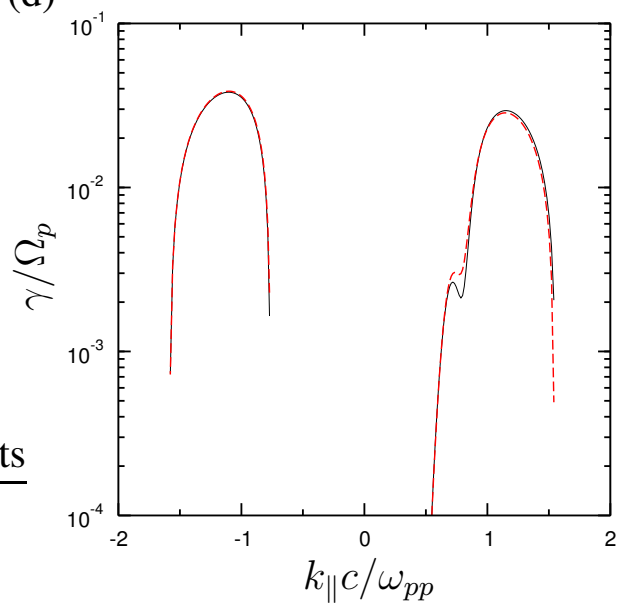

Abbildung 6.2: Die reduzierten Verteilungen (a) nullter und (b) erster Ordnung der Geschwindigkeitsverteilung in der nach Gaußfunktionen entwickelten Form 4.1 (-) und ihre auf maximal $2^{4}$ Summanden verringerten Versionen (---). Die Abbildungen (c) und (d) zeigen den Einfluß dieser Verringerung auf die Dispersionszweige und die Anwachsraten der vorhandenen Instabilitäten. Die verwendete Geschwindigkeitsverteilung der Protonen stammt von Helios 2 und wurde am 14. April 1976 um 10h/46m/8s gemessen.

chen dafür, daß die Verringerung der reduzierten Verteilungen zu keinem bedeutenden Fehler in den aus ihnen berechneten Dispersionsbeziehungen und Anwachsraten führt.

\subsection{Instabilitäten bei schräger Ausbreitungsrichtung}

In diesem Abschnitt sollen die Ergebnisse der Untersuchung des schnellen Sonnenwindes auf Instabilitäten, deren Mode maximaler Anwachsrate sich schräg zum mittleren magnetischen Feld ausbreitet, beschrieben werden. Diese Instabilitäten werden bis auf eine Ausnahme durch den Protonenstrahl verursacht. Es handelt sich um die Alfvén I, II und III Instabilität sowie eine Instabilität der magnetosonischen Moden (s. Abschnitt 2.2.3). Ihnen ist der Abschnitt 6.3.1 gewidmet. Im Abschnitt 6.3.2 komme ich auf ei- 
(a)

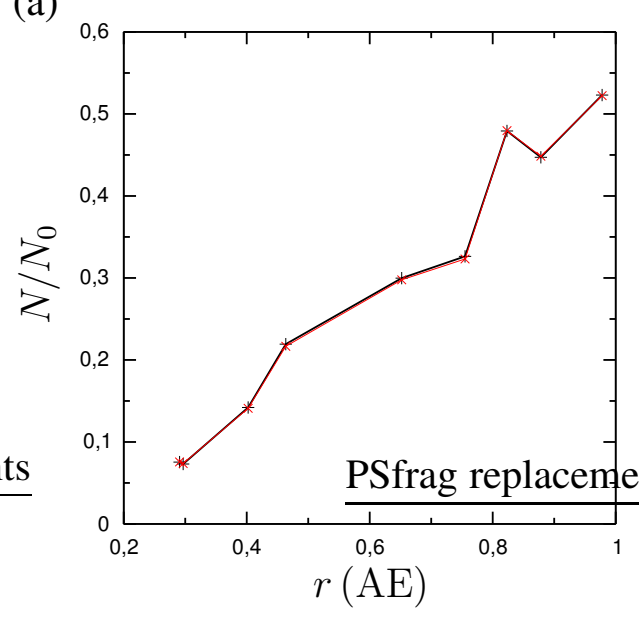

(b)

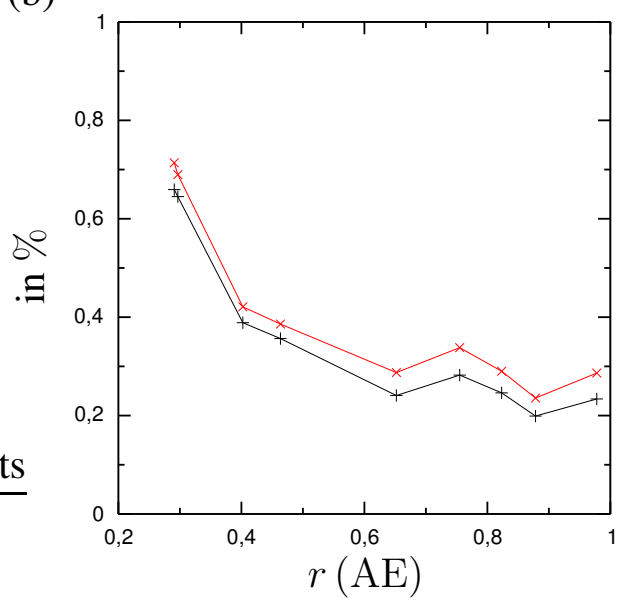

Abbildung 6.3: (a) Der Mittelwert des Verhältnisses aus der verringerten und ursprünglichen Summandenanzahl in Abhängigkeit vom mittleren Abstand der Messungen zur Sonne in den für diese Arbeit ausgewählten Strömungen schnellen Sonnenwindes (s. Tabelle 3.1). (b) Relativer Abstand $\left\|F_{0,1}-\tilde{F}_{0,1}\right\| /\|F\|$ der reduzierten Verteilungen nullter und erster Ordnung mit einer verringerter Anzahl von Summanden $\tilde{F}_{0,1}$ zu den Ausgangsverteilungen $F_{0,1}$. In (a) und (b) beziehen sich die schwarzen und die roten Kurven auf die reduzierten Verteilungen nullter bzw. erster Ordnung.

ne Instabilität zu sprechen, welche nicht vom Protonenstrahl herrührt und sich in wenigen Einzelfällen beobachten ließ: die sogenannte Spiegel-Instabilität. Sie wurde nicht im Abschnitt 2.2.3 über Mikroinstabilitäten diskutiert und kann durch die Temperaturanisotropie des Kerns der Geschwindigkeitsverteilung der Protonen ausgelöst werden. Die für alle Ergebnisse der Abschnitte 6.3.1 und 6.3.2 entscheidende Dispersionsbeziehung hat die im Abschnitt 2.2.2 behandelte Form, worin der Kern und der Strahl der Verteilung der Protonen als unabhängige Bi-Maxwell-Verteilungen eingehen. Diese Verteilungen von Kern und Strahl wurden in jedem Einzelfall durch die im Abschnitt 6.1 beschriebene Kern-Strahl-Zerlegung bestimmt. Als Resultat der Auswertung liegen für jeden kombinierten Datensatz der Protonen, Elektronen und $\alpha$-Teilchen Lösungen der Dispersionsbeziehung auf einem regulären Gitter im Wellenzahlenraum vor. Das Gitter füllt den durch $5 \times 10^{-3} \leq|\vec{k}| c / \omega_{p p} \leq 2.5$ bzw. $0^{\circ} \leq \theta \leq 80^{\circ}$ definierten Bereich aus ${ }^{1}$ und umfaßt $45 \times 25$ Punkte. Der Ausbreitungswinkel $\theta$ bezieht sich im folgenden ebenso wie im Kapitel 2 auf die durch den Protonenstrahl bestimmte Richtung entlang des mittleren magnetischen Feldes. Auf diese Weise wurden alle Dispersionszweige im Bereich der Zyklotronfrequenzen der Ionen berechnet, die die zu Beginn genannten Instabilitäten besitzen können.

\footnotetext{
${ }^{1}$ Alle instabilen Wellenmoden, deren Ausbreitungswinkel größer als $90^{\circ}$ ist, wurden für die Berechnungen durch die Symmetrie $\vec{k} \rightarrow-\vec{k}, \omega \rightarrow-\omega^{*}$ der Dispersionsbeziehung ausgetauscht (s. Abschnitt 2.2.2). In den Darstellungen dieses Abschnittes ist der Ausbreitungswinkel ggf. durch $\theta \rightarrow 180^{\circ}-\theta$ wiederhergestellt worden, um die Konventionen des Abschnittes 2.2.3 einzuhalten.
} 
(a)

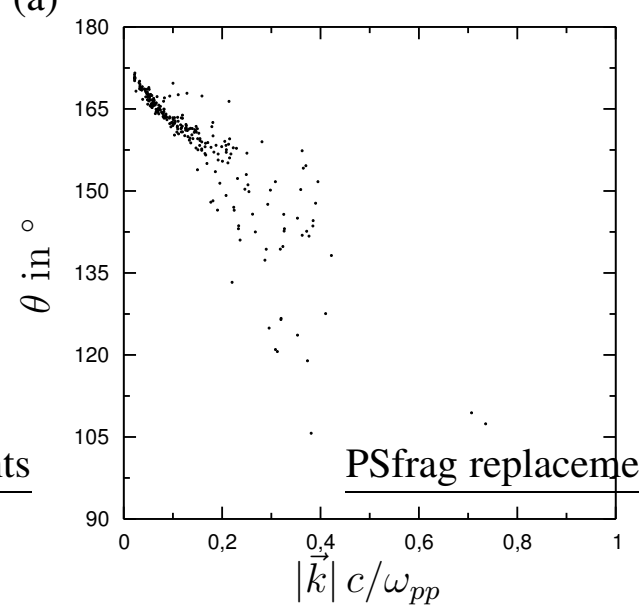

(c)

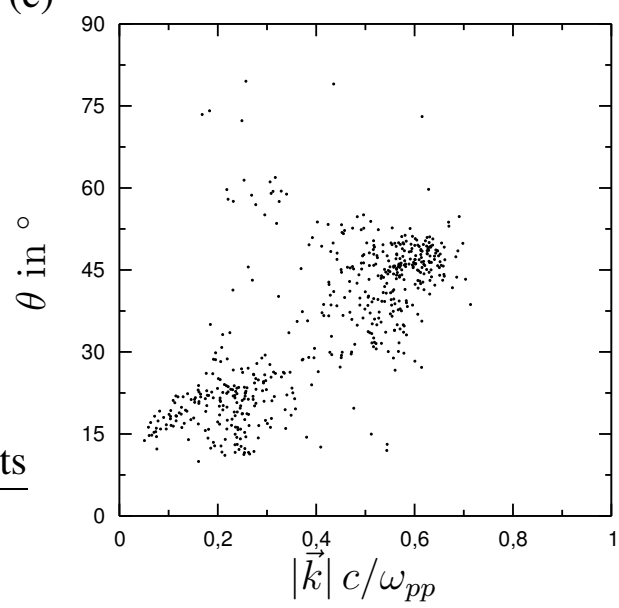

Abbildung 6.4: Verteilung des Wellenvektors der Mode maximaler Anwachsrate der beobachteten (a) magnetosonischen und (b) Alfvén I und II Instabilitäten.

\subsubsection{Strahl-Instabilitäten}

Von den Strahl-Instabilitäten, deren Mode maximaler Anwachsrate sich schräg zum mittleren magnetischen Feld ausbreitet, ließ sich nur die Alfvén III Instabilität nicht nachweisen. Für die Mittelwerte der Parameter der Verteilungen, wie sie in den ausgewerteten schnellen Sonnenwindströmungen gemessenen wurden (s. Tabelle A.1), existiert allerdings keine der Strahl-Instabilitäten. Die Abbildungen 6.4a und 6.4b zeigen Streudiagramme der Wellenvektoren der Moden maximaler Anwachsrate für die aufgefundenen magnetosonischen bzw. die Alfvén I und II Instabilitäten. Die Alfvén I und II Instabilitäten heben sich in getrennten Populationen bei großem Betrag des Wellenvektors, $0.35 \lesssim|\vec{k}| c / \omega_{p p}$, und großem Ausbreitungswinkel, $30^{\circ} \lesssim \theta$, bzw. umgekehrt von einander ab (vgl. Abbildung 2.3b). Die Streudiagramme umfassen 256 magnetosonische sowie 554 Alfvén I und II Instabilitäten, was, bezogen auf die 10804 in der Auswertung enthaltenen Datensätze, den geringen Häufigkeiten von $2.4 \%$ bzw. $5.1 \%$ entspricht. Die Abbildungen 6.5a und 6.5b zeigen normierte Histogramme der maximalen Anwachsraten der magnetosonischen bzw. der Alfvén Instabilitäten. Von den nachgewiesenen 256 magnetosonischen Instabilitäten besitzen 41 eine maximale Anwachsrate oberhalb von $10^{-3}$ und sind deshalb nicht in der Abbildung 6.5a zu sehen. Die maximale Anwachsrate der 554 nachgewiesenen Alfvén Instabilitäten sind in 69 Fällen größer als $5 \times 10^{-3}$ und somit nicht in der Abbildung 6.5b zu erkennen. Diese starken Instabilitäten treten ausnahmslos bei spontanen Einbrüchen des Betrages des mittleren magnetischen Feldes und somit der Alfvén-Geschwindigkeit auf (s. Abbildungen 6.11 und A.2). Die Geschwindigkeitsverteilungen bleiben von diesen Einbrüchen unberührt, weshalb die auf die Alfvén-Geschwindigkeit normierte Relativgeschwindigkeit des Protonenstrahls weit über die Stabilitätsgrenze gehoben wird. Unter Vernachlässigung der nicht in den Histogrammen erkennbaren Fälle betragen die Mittelwerte der maximalen Anwachsraten $\gamma_{m} / \Omega_{p}=1.1 \times 10^{-4}$ und $\gamma_{m} / \Omega_{p}=6.2 \times 10^{-4}$ für die magnetosonische Instabilität bzw. die zusammengefaßten Alfvén I und II Instabilitäten. In der Abbildung 6.5c sind die Häufigkeiten der magnetosonischen Instabilitäten und der Alfvén I und II Instabilitäten in 
(a)

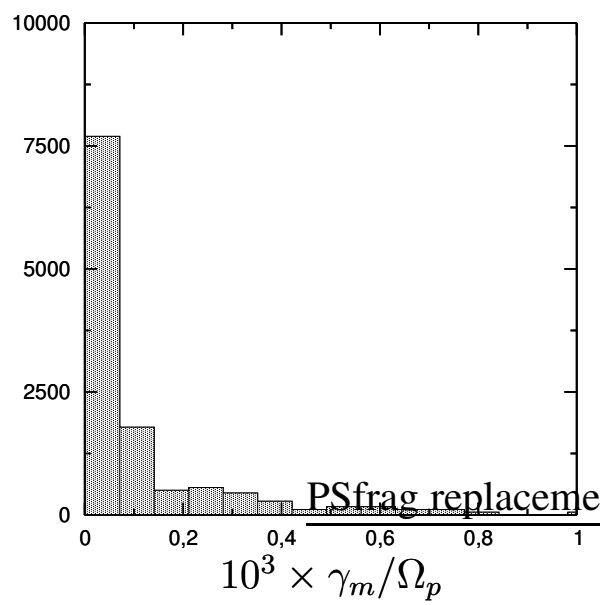

(c)

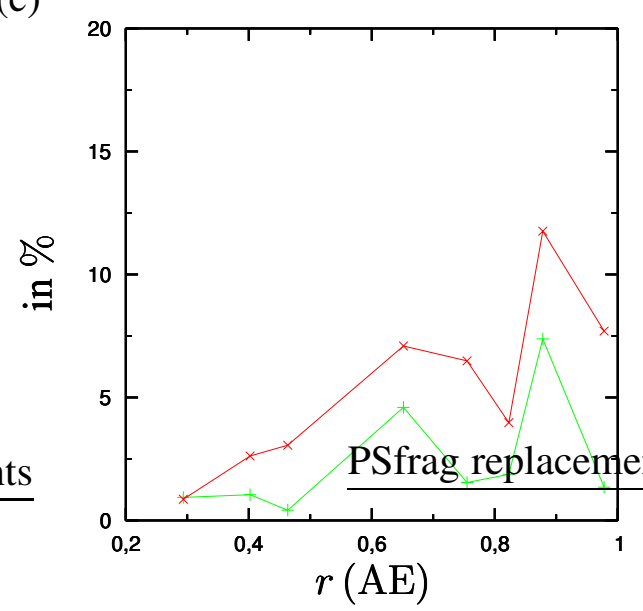

(b)

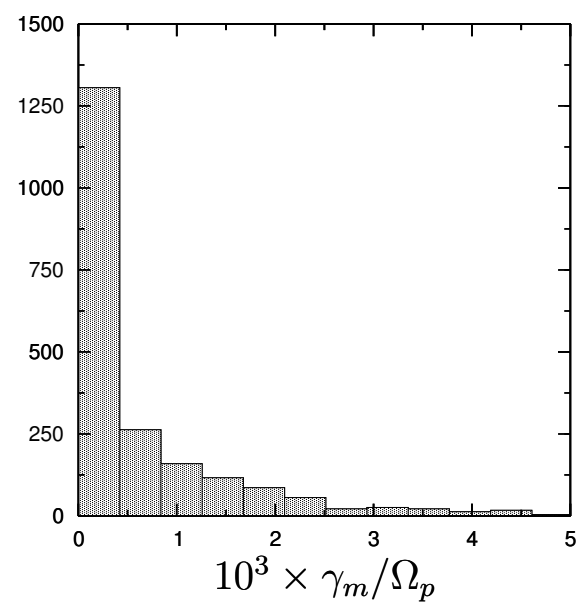

(d)

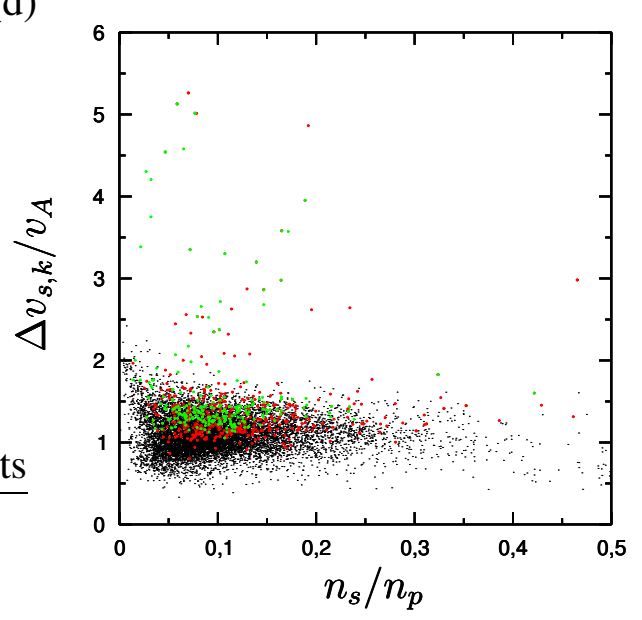

Abbildung 6.5: Normierte Histogramme der maximalen Anwachsraten (a) der magnetosonischen Instabilität sowie (b) der Alfvén I und II Instabilitäten. (c) Häufigkeit der Alfvén I, II (rot) und magnetosonischen (grün) Instabilität in Abhängigkeit von der mittleren Entfernung der betrachteten Strömungen schnellen Sonnenwindes zur Sonne. (d) Streudiagramm der Dichte und der Geschwindigkeit des Protonenstrahls aller ausgewerteten Geschwindigkeitsverteilungen (schwarz), der Verteilungen mit einer magnetosonischen Instabilität (grün) und der Verteilungen mit einer Alfvén I oder II Instabilität (rot).

den ausgewerteten Sonnenwindströmungen über die mittlere Entfernung der Messungen zur Sonne aufgetragen. Es ist ein schwacher Anstieg der Häufigkeiten mit wachsender Entfernung zu erkennen. Die Strahl-Instabilitäten treten allerdings noch im Aphel von Helios 2 mit einer Häufigkeit von höchstens 10\% selten auf. In der Abbildung 6.5d ist zu sehen, daß die instabilen Geschwindigkeitsverteilungen trotz dieser Beobachtungen nicht am Rande der Menge aller Verteilungen liegen. Sie zeigt ein Streudiagramm der Teilchendichte und der mittleren Geschwindigkeit des Protonenstrahls. Die Anwachsraten der Strahl-Instabilitäten werden, nach der in den Abbildungen 2.4a-2.4f dargestellten Parameterstudie (s.a. Daughton und Gary 1998) zu urteilen, im wesentlichen durch diese beiden Parameter bestimmt. Das Auftreten der magnetosonischen bzw. der Alfvén Instabilitäten ist farblich hervorgehoben. Instabile und stabile Geschwindigkeitsverteilungen 
(a)

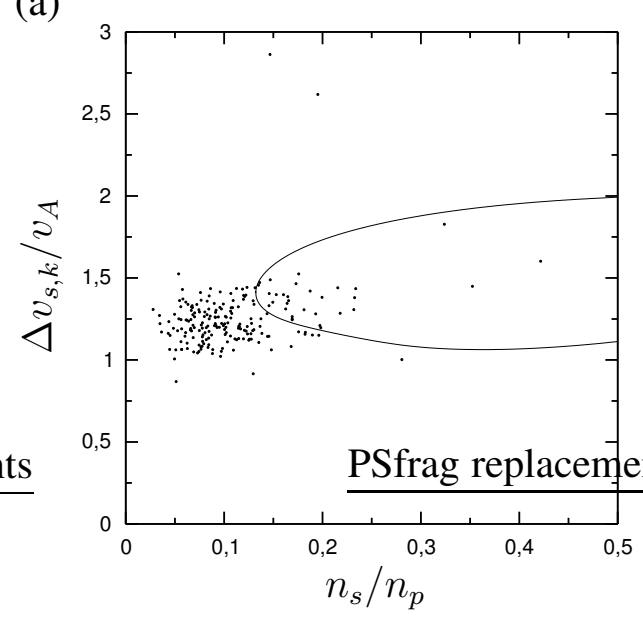

(b)

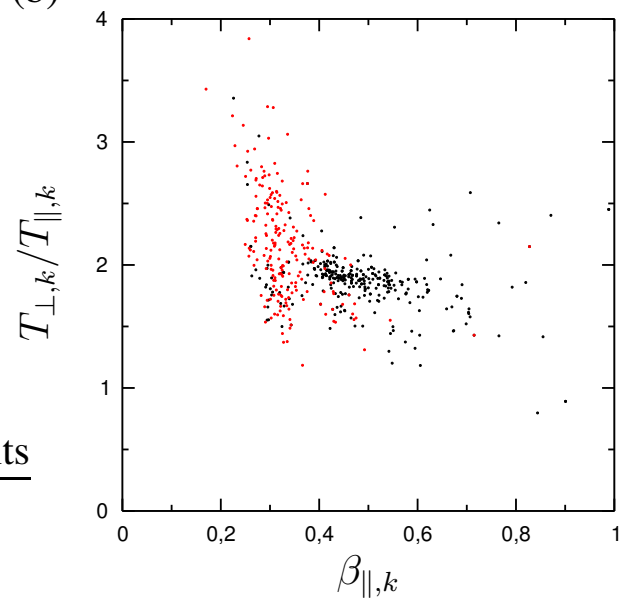

Abbildung 6.6: (a) Streudiagramm der Werte der Dichte und der Geschwindigkeit des Protonenstrahls für die Verteilungen mit einer Alfvén II Instabilität sowie die durch $\gamma_{m} / \Omega_{p}=10^{-5}$ definierte Stabilitätsgrenze der Alfvén II Instabilität, welche auf den Parametern der Tabelle 2.2 beruht. (b) Streudiagramm der Werte des Plasmabetas und der Temperaturanisotropie des Kerns der Verteilung der Protonen für die Fälle mit einer Alfvén I (schwarz) und einer Alfvén II Instabilität (rot).

treten nicht in getrennten Gebieten der Parameterebene auf. Mit Ausnahme der Verteilungen, deren Strahlgeschwindigkeit $\Delta v_{s, k}$ kleiner als die Alfvén-Geschwindigkeit $v_{A}$ ist, könnten sich alle Verteilungen in der Nähe der Grenze absoluter Stabilität befinden. Die Teilchendichte des Protonenstrahls entscheidet nicht, welche der Instabilitätstypen auftritt. $\mathrm{Zu}$ der entgegengesetzten Annahme verleitet hingegen die Abbildung 2.5b, in der Stabilitätsgrenzen der Strahl-Instabilitäten zwischen der Dichte und der Geschwindigkeit des Protonenstrahls zu sehen sind. Demnach sollte die magnetosonische Instabilität bei kleinen Teilchendichten des Protonenstrahls auftreten $\left(n_{s} \lesssim 0.25 n_{p}\right)$, während die Alfvén II Instabilität im umgekehrten Fall zu erwarten wäre. Damit die Alfvén I Instabilität mit vergleichbaren Anwachsraten wie die magnetosonische oder die Alfvén II Instabilität auftritt, sollten zudem größere Geschwindigkeiten des Protonenstrahls erforderlich sein. Nur die magnetosonischen Instabilitäten treten in dem Parameterbereich auf, in dem sie aufgrund der Parameterstudie des Abschnittes 2.2.3 mit den beobachteten Anwachsraten zu erwarten sind (s. Abbildungen 2.4e und 2.4f). Für eine getrennte Untersuchung der sich auf demselben Dispersionszweig befindenden Alfvén I und II Instabilitäten habe ich die beobachteten Fälle anhand der Abbildung 6.4b aufgeteilt. Die Frequenz, die Polarisation oder die Anwachsrate der Moden maximaler Anwachsrate erlauben hingegen keine Unterscheidung beider Instabilitätstypen. Ich betrachte alle Alfvén-Instabilitäten, die sich unterhalb der Geraden befinden, welche die Achsenabschnitte 0.7 und $60^{\circ}$ verbindet, als Alfvén II Instabilitäten und die verbleibenden als Alfvén I Instabilitäten. Dieses Vorgehen führt allerdings zu keiner sicheren Unterscheidung der Instabilitätstypen. Für wenige Fälle in der Nähe der Trennlinie mag eine falsche Zuordnung getroffen worden sein. Die Mittelwerte der maximalen Anwachsrate betragen demnach $\gamma_{m} / \Omega_{p}=1.0 \times 10^{-3}$ und $\gamma_{m} / \Omega_{p}=8.8 \times 10^{-5}$ für 281 Alfvén I bzw. 204 Alfvén II Instabilitäten. Fälle mit maximalen Anwachsraten oberhalb von $\gamma_{m} / \Omega_{p}=5 \times 10^{-3}$ wurden dabei ausgelas- 


\begin{tabular}{ccccc}
\hline \hline Parameter & Protonen-Kern & Protonen-Strahl & $\alpha$-Teilchen & Elektronen \\
\hline$n / n_{p}$ & 0.96 & 0.04 & 0.02 & 1.04 \\
$T_{\|} / T_{\|, k}$ & 1 & 1.23 & 6.76 & 0.74 \\
$T_{\perp} / T_{\|}$ & 1.9 & 1.4 & 1 & 1 \\
\hline \hline
\end{tabular}

Tabelle 6.1: Aus den auf Helios 2 am 6. März 1976 um 14h/16m/49s gemessenen Geschwindigkeitsverteilungen der Protonen, Elektronen und $\alpha$-Teilchen abgeleitete Plasmaparameter. Desweiteren sind $v_{A} / c=3.1 \times 10^{-4}, \beta_{\|, k}=0.41, \Delta v_{k, s}=1.59 v_{A}$, $\Delta v_{k, \alpha}=0.93 v_{A}$ und $T_{\alpha} / T_{p}=3.8$. Die genannten Parameter führen zu einer Alfvén I Instabilität.

sen. Diese entsprechen sehr kleinen Beträgen des magnetischen Feldes bzw. der AlfvénGeschwindigkeit $\left(v_{A} / c \lesssim 2 \times 10^{-4}\right.$, vgl. Abbildung A.2b). In der Abbildung 6.6a ist die Stabilitätsgrenze der Alfvén II Instabilität zwischen der Teilchendichte und der Geschwindigkeit des Protonenstrahls zu der beobachteten Größenordnung $\gamma_{m} / \Omega_{p}=10^{-5}$ ihrer maximalen Anwachsrate zusammen mit dem Streudiagramm der Parameterwerte dargestellt (vgl. Abbildungen 2.5b und 6.5d). Die der Stabilitätsgrenze zugrunde liegenden Parameter sind in der Tabelle 2.2 zusammengefaßt. Es ist zu erkennen, daß die Stabilitätsgrenze die gemessenen Parameterwerte erreicht hat. Die Abbildungen 6.7a-6.7c erklären, weshalb die Mehrheit der Werte dennoch im Bereich der Parameterebene zu Anwachsraten unterhalb von $\gamma_{m} / \Omega_{p}=10^{-5}$ anzutreffen sind. Sie zeigen insbesondere die Abhängigkeit der maximalen Anwachsrate der Alfvén II Instabilität von der Temperatur des Protonenstrahls parallel zum magnetischen Feld und von seiner Temperaturanisotropie (vgl. Abbildungen 2.4a-2.4d). Die Temperaturanisotropie des Protonenstrahls und das Verhältnis von seiner Temperatur parallel zum magnetischen Feld zu derjenigen des Kerns sind in den ausgewerteten Strömungen schnellen Sonnenwindes in der deutlichen Mehrheit größer als Eins (s. Abbildungen A.3b, A.3f und Tabelle A.1), und die maximale Anwachsrate der Alfvén II Instabiltät nimmt mit diesen Parametern zu. Die gezeigte Stabilitätsgrenze berücksichtigt diese Tatsache nicht (s. Tabelle 2.2).

Die Abbildung 6.6b verdeutlicht, von welchen Parametern der Verteilung der Protonen das Auftreten der Alfvén I Instabilität abhängt. Sie zeigt ein Streudiagramm der Temperaturanisotropie und des Plasmabetas $\beta_{\|, k}$ des Kerns der gemessenen Verteilungen für diejenigen Fälle, in denen sich eine Alfvén I bzw. eine Alfvén II Instabilität nachweisen ließ. Die Alfvén I Instabilitäten treten nahezu ausschließlich bei Temperaturanisotropien $T_{\perp, k} / T_{\|, k} \approx 2$ und Plasmabetas $0.4 \lesssim \beta_{\|, k}$ auf. Der Wert $\beta_{\|, k} \approx 0.4$ trennt Alfvén I und II Instabilitäten (s. dazu auch Abbildung 6.7d). Die Plasmaparameter der Tabelle 6.1 erlauben es, diesen Sachverhalt genauer zu beleuchten. Sie gehören zu Geschwindigkeitsverteilungen der Protonen, Elektronen und $\alpha$-Teilchen, die auf Helios 2 gemessenen wurden, und führen zu einer Alfvén I Instabilität. In der Abbildung 6.8a sind Höhenlinien der Anwachsrate der instabilen Alfvén-Moden in Abhängigkeit von dem Ausbreitungswinkel und dem Betrag des Wellenvektors zu sehen. Bei paralleler Ausbreitungsrichtung tritt zusätzlich die von der Temperaturanisotropie des Kerns der Protonen verursachte Instabilität auf (vgl. Abbildungen 2.3a und 2.3b). Die Abbildung 6.8b zeigt die Abhängig 
(a)

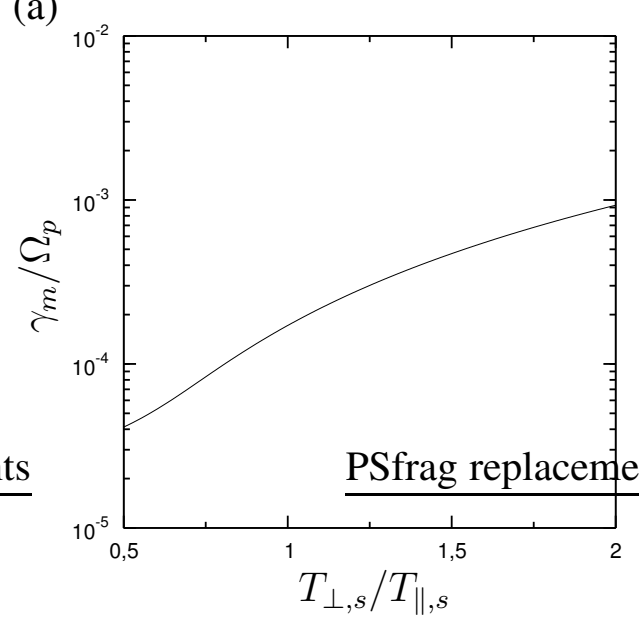

(c)

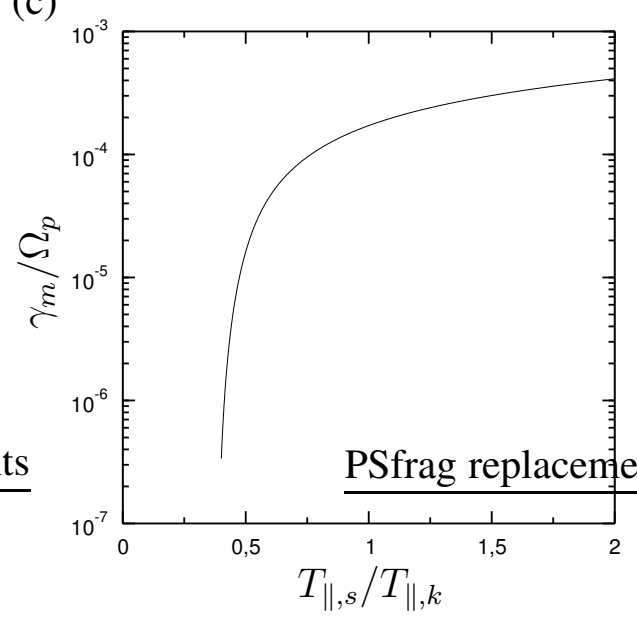

(b)

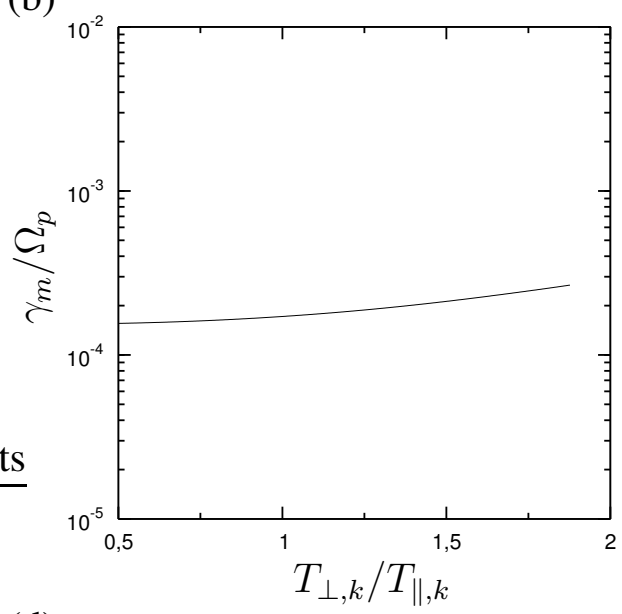

(d)

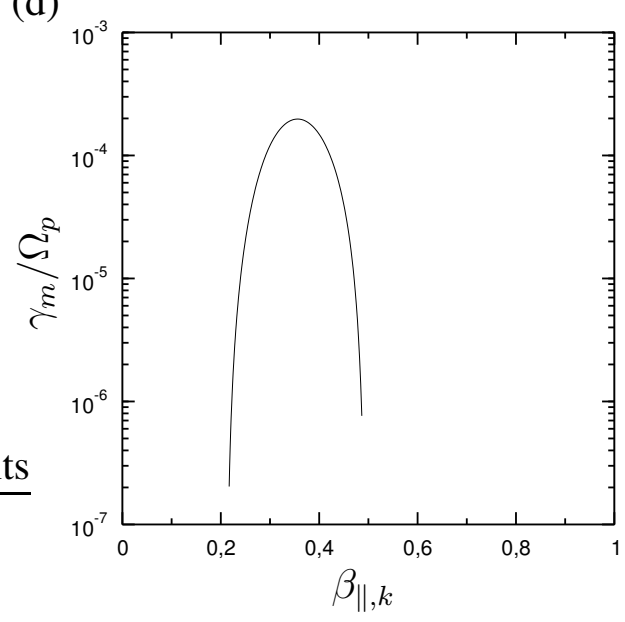

Abbildung 6.7: Abhängigkeit der maximalen Anwachsrate der Alfvén II Instabilität von (a) $T_{\perp, s} / T_{\|, s}$, (b) $T_{\perp, k} / T_{\|, k}$, (c) $T_{\|, s} / T_{\|, k}$ und (d) $\beta_{\|, k}$. Es liegen (a)-(d) die Parameter der Tabelle 2.2 zugrunde (vgl. Abbildungen 2.4a-2.4d).

der Mode maximaler Anwachsrate der Instabilität der Alfvén-Moden bei schräger Ausbreitungsrichtung von der Geschwindigkeit des Protonenstrahls. Durch den Vergleich mit der Abbildung 2.4f wird belegt, daß es sich bei dieser Instabilität tatsächlich um die in den Grundlagen diskutierte Alfvén I Instabilität handelt. Die folgenden Abbildungen 6.8c und 6.8d erklären schließlich, die in der Abbildung 6.6b gezeigte Verteilung der Alfvén I Instabilitäten in Abhängigkeit von der Temperaturanisotropie und dem Plasmabeta $\beta_{\|, k}$ des Kerns der Geschwindigkeitsverteilung der Protonen. Für die Parameter der Tabelle 6.1 nimmt die maximale Anwachsrate der Alfvén I Instabilität zusammen mit der Anisotropie sowie dem Plasmabeta ab und hört bei bestimmten Grenzwerten dieser Parameter auf zu existieren. Im Unterschied zu dem im Abschnitt 2.2.3 diskutierten Fall einer Alfvén I Instabilität, können die in den ausgewerteten Strömungen des schnellen Sonnenwindes bei deutlich kleineren Dichten und Geschwindigkeiten des Protonenstrahls beobachteten Alfvén I Instabilitäten nicht ohne eine Temperaturanisotropie des Kerns bestehen. Insofern handelt es sich bei ihnen um keine eindeutigen Strahl-Instabilitäten mehr.

Aus der der Größenordnung der maximalen Anwachsraten und den geringen Häufigkeiten 
(a)

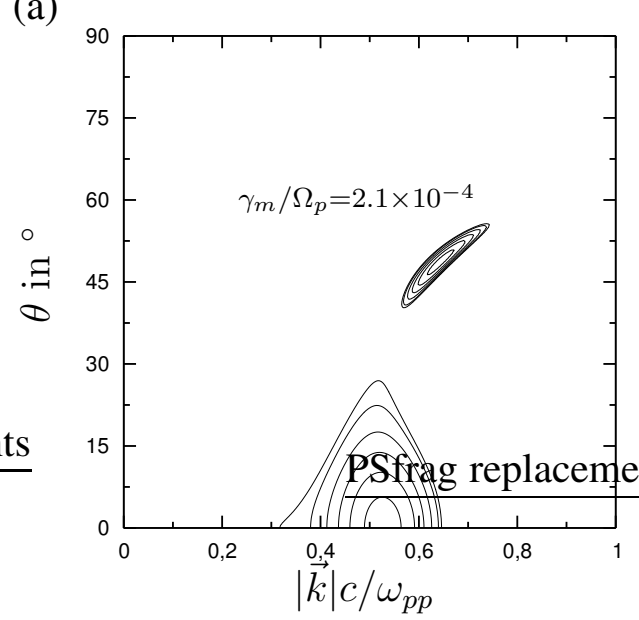

(c)

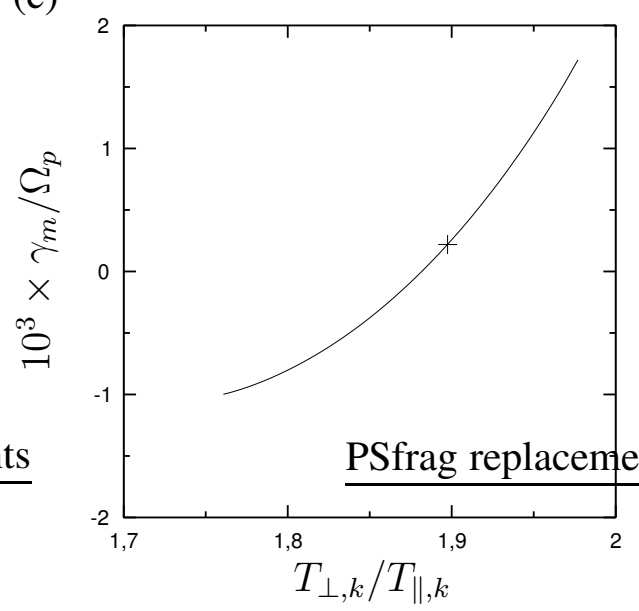

(b)

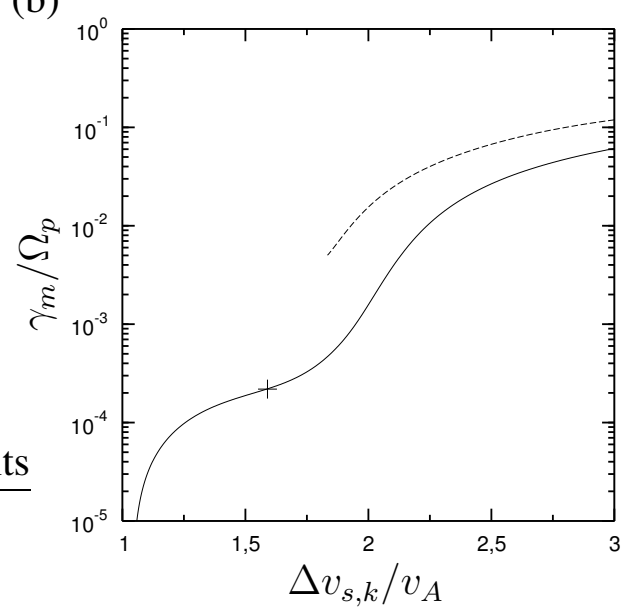

(d)

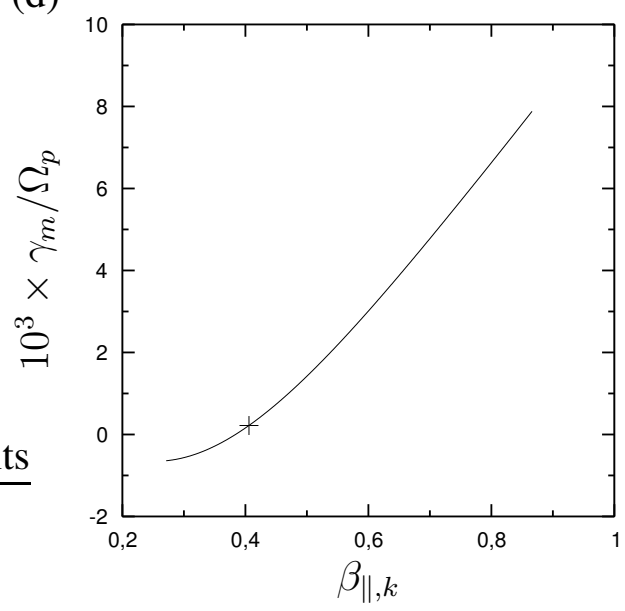

Abbildung 6.8: (a) Höhenlinien der sich aus den in der Tabelle 6.1 genannten Plasmaparametern ergebenden Alfvén I Instabilität und der ebenfalls vorhandenen und von der Temperaturanisotropie des Kerns der Verteilung der Protonen verursachten Instabilität, deren Mode maximaler Anwachsrate sich parallel zum mittleren magnetischen Feld ausbreitet. Die Höhenlinien entsprechen jeweils den Anteilen 0.9, 0.7, 0.5, 0.3, 0.1 und 0 der maximalen Anwachsrate. (b) Abhängigkeit der maximalen Anwachsrate der in (a) gezeigten Alfvén I Instabilität von der Geschwindigkeit des Protonenstrahls. Die gestrichelte Kurve zeigt dieselbe Abhängigkeit, die sie sich aus den Mittelwerten der Plasmaparameter in derjenigen Strömung des schnellen Sonnenwindes ergibt, in der die in der Tabelle 6.1 genannten Parameter gemessen wurden. (c) und (d) zeigen die Abhängigkeit der maximalen Anwachsrate der in (a) gezeigten Alfvén I Instabilität von der Temperaturanisotropie bzw. des Plasmabetas des Kerns der Verteilung der Protonen.

der Strahl-Instabilitäten bei schräger Ausbreitungsrichtung läßt sich schlußfolgern, daß sie im mittleren Zustand des schnellen Sonnenwindes allenfalls marginal instabil sind (s. Diskussion im Abschnitt 2.2.3). Für die Mittelwerte der Plasmaparameter in den ausgewerteten Strömungen des schnellen Sonnenwindes, die jeweils den Zeitraum eines Tages umfassen, existiert keine dieser Strahl-Instabilitäten. Ihre Häufigkeit nimmt mit der Entfernung zur Sonne nur schwach zu. Das Auftreten der Alfvén I Instabilität wird durch 
vergleichsweise kleine Temperaturanisotropien und große Werte von $\beta_{\|, k}$ des Kerns der Geschwindigkeitsverteilung der Protonen begünstigt, die erst nahe der Entfernung von 1 AE im Mittel erreicht werden. Das Anwachsen der Häufigkeit der magnetosonischen Instabilität hängt mit der Zunahme des Mittelwertes von $\Delta v_{s, k} / v_{A}$ mit der Entfernung zur Sonne zusammen (s. Abbildung A.2c). Abschließend stellt sich die Frage, ob die beobachteten Strahl-Instabilitäten im mittleren Zustand des schnellen Sonnenwindes marginal instabil sind oder noch in der Entfernung von $1 \mathrm{AE}$ nicht auftreten. Die marginale Instabilität der Strahl-Instabilitäten wäre mit ihrer (quasi-linearen) Relaxation im Verlauf der Expansion des Windes verbunden. Als Ursache der magnetosonischen ${ }^{2}$ und der Alfvén I Instabilitäten, welche während der Expansion anhält, kommt ggf. die Abnahme der Magnetisierung des Sonnenwindes mit der Entfernung zur Sonne in Betracht. Sie drückt sich durch die Abnahme der Alfvén-Geschwindigkeit aus. Eine Relaxation der Instabilitäten zeigt sich möglicherweise in der Verteilung der gemessenen Plasmaparameter in Abhängigkeit von der Entfernung zur Sonne. Aus diesem Grund sind in den Abbildungen 6.9a-6.9h Streudiagramme der beobachteten Werte der Dichte und der Geschwindigkeit des Protonenstrahls zusammen mit Stabilitätsgrenzen aller Strahl-Instabilitäten in dieser Parameterebene zu sehen. Ein Vergleich dieser Parametern mit Stabilitätsgrenzen wurde auch von Marsch und Livi (1987) und Goldstein et al. (2000) vorgenommen. Dies geschah allerdings mit dem Ziel über die Instabilität der gemessenen Verteilungen zu entscheiden, was in dieser Arbeit durch die direkte Berechnung der Anwachsraten durchgeführt wurde. Die Teilabbildungen unterscheiden zwischen den ausgewerteten Strömungen des schnellen Sonnenwindes. Den Stabilitätgrenzen (vgl. Abbildung 2.5b) liegen jeweils die in der Tabelle A.1 zusammengefaßten Mittelwerte der Plasmaparameter in den Strömungen zugrunde $^{3}$. Die gemessenen Werte der Dichte und der Geschwindigkeit des Protonenstrahls befinden sich in allen Entfernungen zur Sonne unterhalb der Stabilitätgrenzen, was aufgrund der vergleichsweise kleinen Größenordnung der beobachteten Anwachsraten und der geringen Häufigkeit der Strahl-Instabilitäten zu erwarten war. In der Abbildung 6.9a, die zu einer Entfernung von 0.29 AE gehört, ist kein Zusammenhang zwischen der Verteilung der Parameter und den Stabilitätsgrenzen erkennbar. Bei den in diesem Streudiagramm gezeigten Parameterwerten handelt es sich in der Mehrheit um die nur kaum von Instabilitäten durchsetzte Teilmenge des Steudiagramms 6.5c mit Geschwindigkeiten des Protonenstrahl, die kleiner als die Alfvén-Geschwindigkeit sind. Mit wachsender Entfernung zur Sonne ist in den folgenden Abbildungen 6.9b-6.9h zu erkennen, daß sich die Verteilung der Parameterwerte zu größeren Geschwindigkeiten des Protonenstrahls ver-

\footnotetext{
${ }^{2}$ Die magnetosonische Strahl-Instabilität bei paralleler Ausbreitungsrichtung ist ebenfalls betroffen (s. Abschnitt 6.4 zu der direkten Beobachtung ihrer Häufigkeit und maximalen Anwachsraten).

${ }^{3}$ Die Stabilitätsgrenze der Alfvén II Instabilität reicht in der Nähe zum Perihel von Helios 2 bei niedrigen Geschwindigkeiten des Protonenstrahls nicht bis $n_{s} / n_{p}=0.5$ zurück. Die Alfvén II Instabilität tritt nicht oberhalb, sondern im Inneren dieser im Idealfall geschlossenen Kurve auf. In größerer Nähe zur Sonne schließt sich die Stabilitätskurve nicht, weil die Alfvén II Instabilität von der durch die Temperaturanisotropie des Kerns der Verteilung der Protonen verursachten Instabilität der Alfvén-Moden überlagert wird und ihre Anwachsraten bei zu geringen Strahlgeschwindigkeiten kein lokales Maximum besitzen können. Es wurde der Wert $\gamma_{m} / \Omega_{p}=5 \times 10^{-4}$ für die Berechnung der Stabilitätskurven verwendet. Auch die Alfvén I Instabilität wird von der durch die Temperaturanisotropie verursachten Instabilität überlagert, weshalb in den Abbildungen 6.9a-6.9h die ansteigenden Werte $\gamma_{m} / \Omega_{p}=10^{-3}, 10^{-3}, 10^{-3}, 10^{-2}, 10^{-2}, 2 \times 10^{-2}, 2 \times$ $10^{-2}, 3 \times 10^{-2}$ der maximalen Anwachsrate für die Berechnung ihrer Stabilitätsgrenzen verwendet werden mußten. Die Stabilitätsgrenzen aller verbleibenden Strahl-Instabilitäten sind in den Abbildungen 6.9a-6.9h durch den Wert $\gamma_{m} / \Omega_{p}=5 \times 10^{-3}$ definiert.
} 
(a)

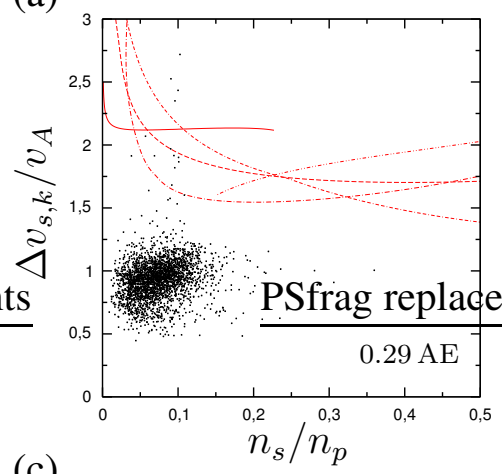

(c)

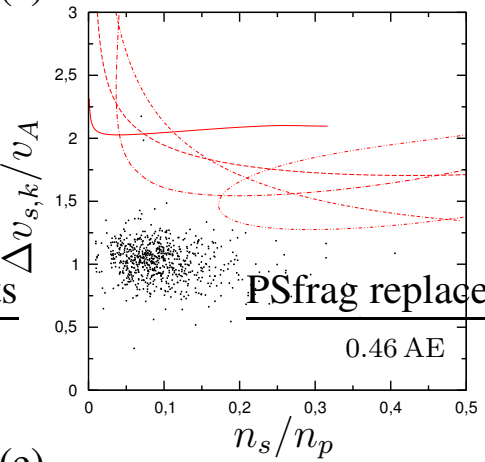

(e)

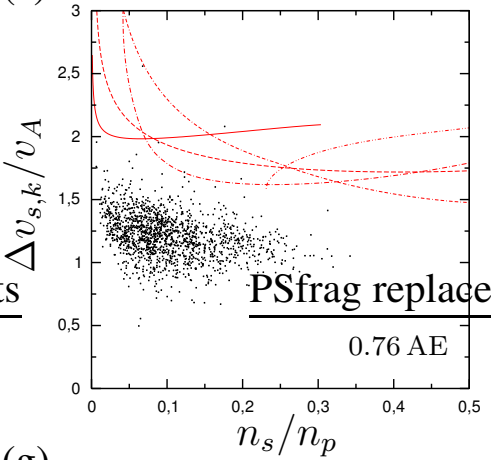

(g)

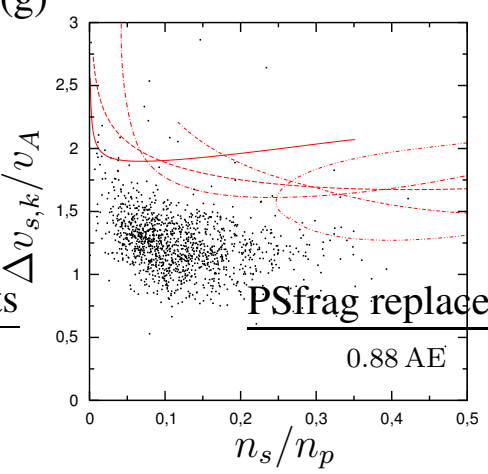

(b)

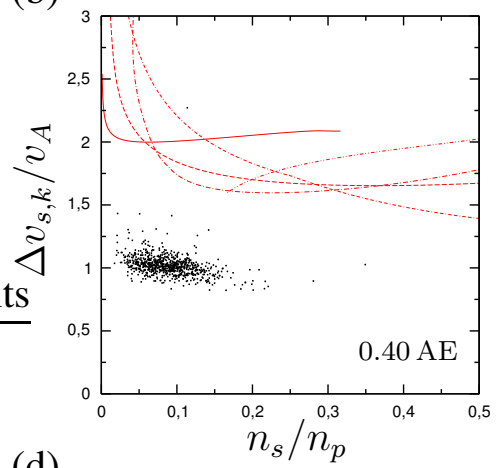

(d)

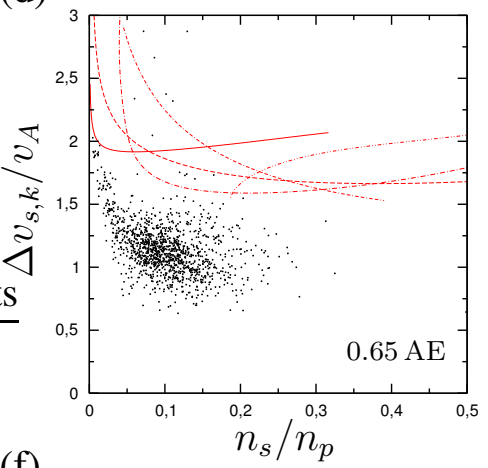

(f)

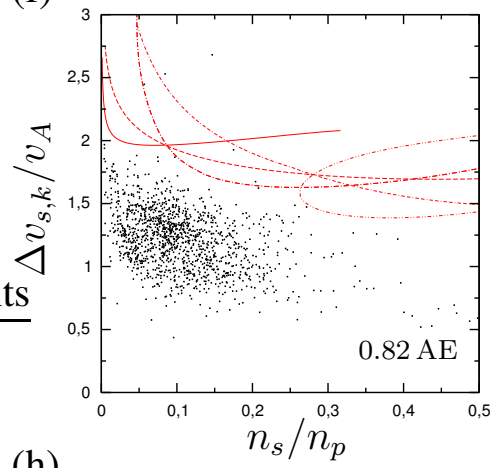

(h)

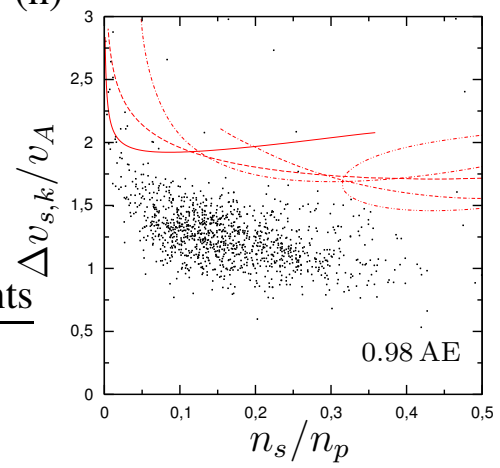

Abbildung 6.9: (a)-(f) Stabilitätsgrenzen der Strahl-Instabilitäten in der aus der Dichte und der Geschwindigkeit des Protonenstrahls gebildeten Parameterebene (parallel magnetosonisch (-), Alfvén I (---), schräg magnetosonisch (---), Alfvén II (--.-), Alfvén III (.-.)) sowie Streudiagramme der gemessenen Werte dieser Parameter. Die Teilabbildungen unterscheiden nach den ausgewerteten Strömungen des schnellen Sonnenwindes (s. Tabelle 3.1). Den Stabilitätsgrenzen liegen jeweils die Mittelwerte der Plasmaparameter aus diesen Strömungen (s. Tabelle A.1) und die in der Fußnote 3 genannten Werte der maximalen Anwachsrate zugrunde. 
schiebt und die Teilchendichte des Strahl stärker streut. Dabei verteilen sich die Parameter in zunehmendem Maße entlang der Stabilitätskurven. Diese Beobachtung kann als schwaches Indiz für eine Relaxation von Strahl-Instabilitäten im mittleren Zustand des schnellen Sonnenwindes betrachtet werden. In jedem Fall ist sie wie die bisherigen Ergebnisse mit der Vorstellung einer quasi-linearen Relaxation der Strahl-Instabilitäten verträglich, welche somit nicht ausgeschlossen werden kann. Daughton et al. (1999) führten numerische Simulationen der Relaxation der Alfvén I und der magnetosonischen Instabilität bei paralleler Ausbreitungsrichtung aus. Sie fanden, daß die Relaxation beider Instabilitäten zur Erhöhung der Temperaturanisotropie des Protonenstrahls und im Fall der Alfvén I Instabilität auch zur Erhöhung der Temperaturanisotropie des Kerns der Geschwindigkeitsverteilung der Protonen führt (vgl. Schwartz et al. (1981)). Letzteres ist möglich, da Wellenmoden, die sich schräg zum mittleren magnetischen Feld ausbreiten, über alle Resonanzen mit den Teilchen wechselwirken (s. Gleichungen 2.40-2.43) und die Moden der Alfvén I Instabilität eine Zyklotron-Resonanz im Kern der Verteilung der Protonen besitzen. Als Resultat ihrer Simulationen erhielten Daughton et al. (1999) stets größere Temperaturanisotropien des Protonenstrahls, $T_{s \perp} / T_{s \|}>T_{k \perp} / T_{k \|}$. Dafür läßt sich in den in dieser Arbeit untersuchten Daten kein Anzeichen finden (s. Anhang A), was somit gegen eine Relaxation von Strahl-Instabilitäten im mittleren Zustand des Sonnenwindes spricht. Die Anwendbarkeit ihrer Simulationen ist allerdings fraglich, da sie anfängliche Temperaturanisotropien vernachlässigten und insbesondere von sehr großen Anwachsraten der Strahl-Instabilitäten ausgingen, welche den Ergebnisse dieser Arbeit widersprechen. Dabei beriefen sie sich insbesondere auf Marsch und Livi (1987), die bei ihrer Auswertung von Messungen des Plasmaexperimentes auf Helios eine große Menge von Verteilungen der Protonen mit Geschwindigkeiten des Protonenstrahls oberhalb von $2 v_{A}$ gefunden haben (s.a. Marsch et al. 1982c). Die von ihnen ermittelte Verteilung der Strahlgeschwindigkeit stimmt nicht mit meinen Ergebnissen überein. Dafür stehen meine Ergebnisse im vollständigen Einlang mit denjenigen der Arbeit von (Tu et al. 2004), die ebenfalls von Helios 2 stammende Messungen aus dem schnellen Sonnenwind in Hinsicht auf den Protonenstrahl untersucht haben. Ich schließe mich der dort geäußerten Meinung an, daß die Resultate von (Marsch und Livi 1987) zu großen Teilen auf eine falsche Identifikation des Protonenstrahls innerhalb der Verteilung der Protonen zurückzuführen sind.

\subsubsection{Spiegel-Instabilität}

Einige wenige und nahezu ausschließlich aus der Nähe des Perihels von Helios stammende Geschwindigkeitsverteilungen weisen bei schräger Ausbreitungsrichtung die SpiegelInstabilität (Hasegawa 1969, Gary 1993, Southwood und Kivelson 1993) auf. Sie entsteht durch die Temperaturanisotropie $T_{\perp}>T_{\|}$des Kerns der Geschwindigkeitsverteilung der Protonen und wird durch große Werte von $\beta_{\|, k}$ begünstigt. Die Frequenz der Moden dieser Instabilität verschwindet im Bezugssystem des Kerns der Verteilung der Protonen. In dem mit dem Massenschwerpunkt aller Teilchen mitbewegten Bezugssystem, gilt somit $\omega \approx v_{\|, k}|\vec{k}| \cos \theta$, wobei $v_{\|, k}$ die mittlere Geschwindigkeit des Kerns der Verteilung der Protonen in diesem Bezugssystem ist. Die Abbildung 6.10a zeigt Höhenlinien einer der ausgewerteten Geschwindigkeitsverteilungen der Protonen. In der Abbildung 6.10b sind Höhenlinien der durch die Kern-Strahl-Zerlegung (s. Abschnitt 6.1) vereinfachten 
(a)

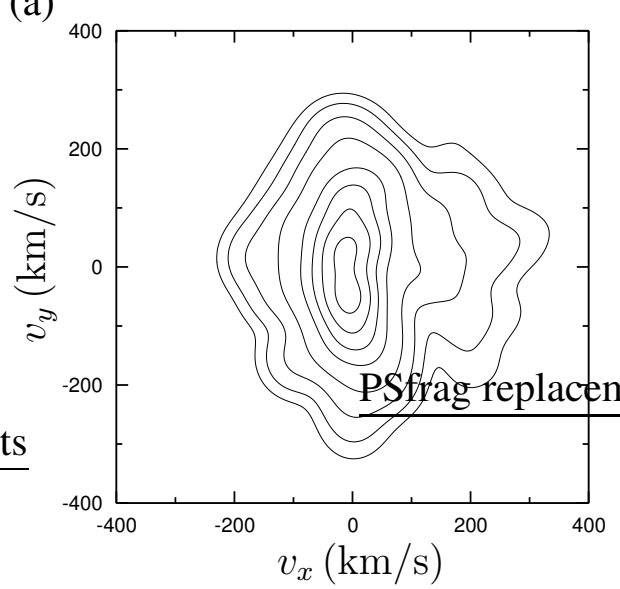

(b)

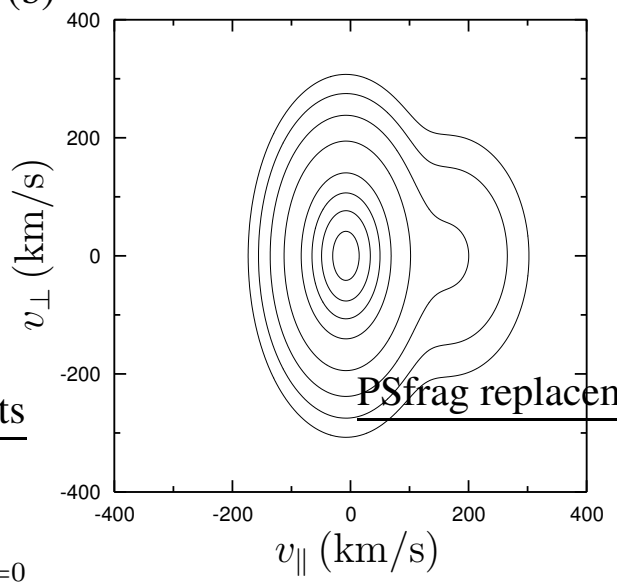

(c)

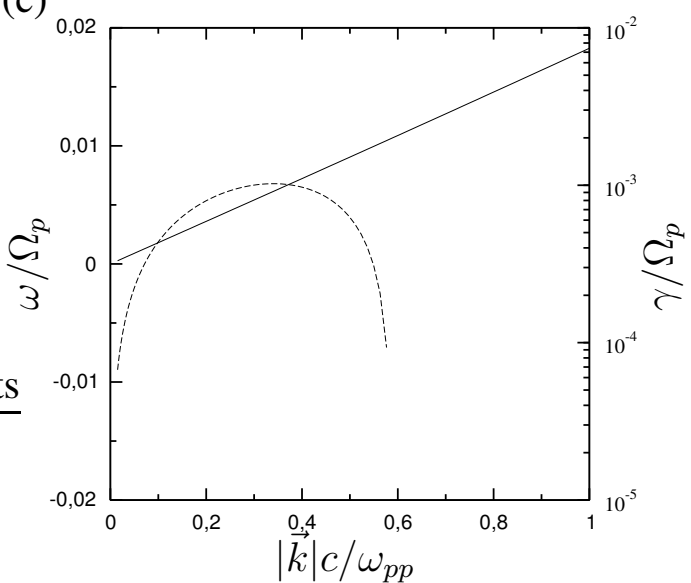

(d)

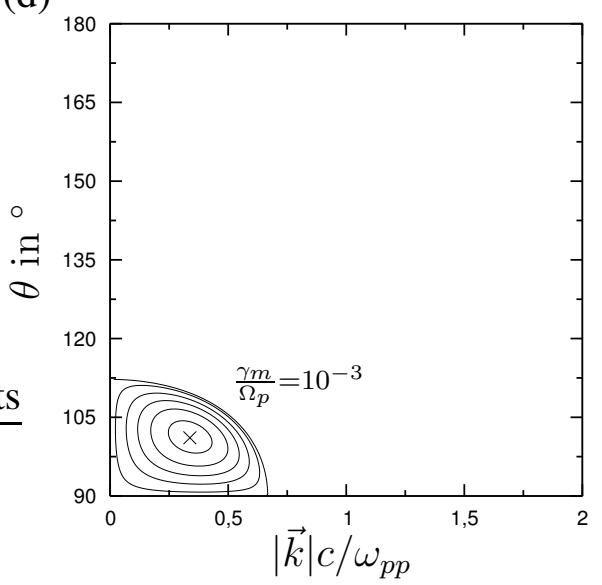

Abbildung 6.10: (a) Höhenlinien der Entwicklung 4.1 einer auf Helios 2 am 14. April 1976 um 9h/5m/33s gemessenen Geschwindigkeitsverteilung der Protonen, die zu einer Spiegel-Instabilität führt. (b) Höhenlinien der Kern-Strahl-Approximation dieser Verteilung. Die Höhenlinien entsprechen jeweils den Anteilen 0.9, 0.7, 0.5, 0.3, 0.1, 0.03, 0.01 und 0.003 des Maximums der Verteilung. Die Abszisse ist parallel zur Richtung des mittleren magnetische Feldes. Der Ursprung stimmt mit der mittleren Geschwindigkeit der Verteilung überein. (c) Frequenz und Anwachsrate des instabilen Wellenmoden in Abhängigkeit vom Betrag des Wellenvektors bei der Ausbreitungsrichtung $\theta=101.3^{\circ}$ der Mode maximaler Anwachsrate. (d) Höhenlinien der Anwachsrate der Spiegel-Instabilität im Wellenzahlraum, die den Anteilen 0.9, 0.7, 0.5, 0.3, 0.1 und 0 der maximalen Anwachsrate entsprechen.

Verteilung zu sehen. Die aus ihr und den gleichzeitig gemessenen Verteilungen der Elektronen und $\alpha$-Teilchen ermittelten Plasmaparameter sind in der Tabelle 6.2 zusammengestellt. Sie führen zu einer der beobachteten Spiegel-Instabilitäten. Die Abhängigkeit der Frequenz und der Anwachsrate der instabilen Moden vom Betrag des Wellenvektors sind in der Abbildung 6.10c für den Ausbreitungswinkel der Mode maximaler Anwachsrate dargestellt. Höhenlinien der Anwachsraten der Moden dieser Spiegel-Instabilität in Abhängigkeit vom Betrag des Wellenvektors und dem Ausbreitungswinkel sind in der Abbildung 6.10d zu sehen. Die Auswertung ergab insgesamt 54 Spiegel-Instabilitäten, von 


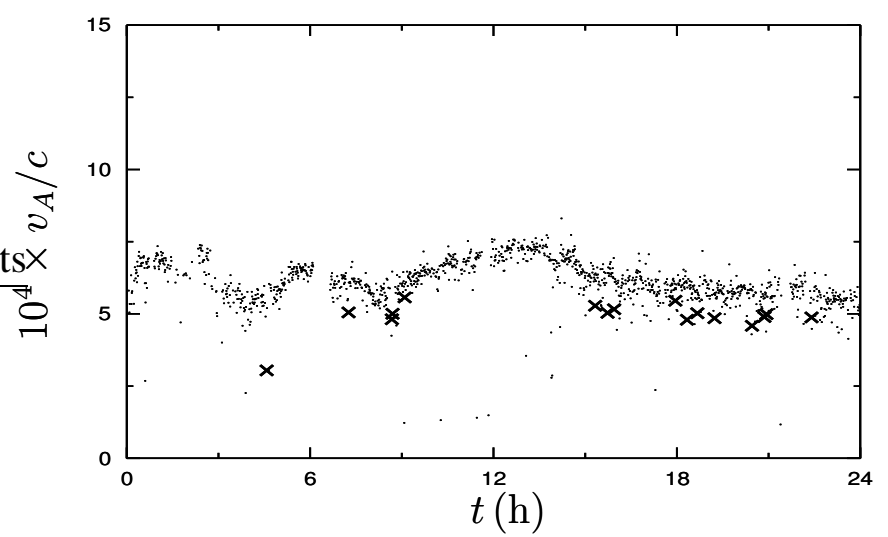

Abbildung 6.11: Zeitlicher Verlauf der Alfvén-Geschwindigkeit in der Sonnenwindströmung des 14. Aprils 1976. Das Auftreten von Spiegel-Instabilitäten ist mit Kreuzen markiert.

denen 30 aus den Sonnenwindströmungen vom 14. und 16. April 1976 aus der Entfernung von 0.29 AE stammen. Anhand der Abbildung 6.11 kann eine Ursache für das Auftreten der Spiegel-Instabilität erkannt werden. Sie zeigt den zeitlichen Verlauf des Plasmabetas und der Temperaturanisotropie des Protonenkerns sowie der normierten AlfvénGeschwindigkeit, wie er auf Helios am 14. April 1976 in der Nähe des Perihels gemessen wurde. Die nachgewiesenen Spiegel-Instabilitäten sind unter den Werten mit Kreuzen markiert. Es ist zu erkennen, daß sie auftreten, wenn die Alfvén-Geschwindigkeit wegen eines zeitlich begrenzten Abfalls des Betrages des mittleren magnetischen Feldes unter ihren Umgebungswert sinkt. Da die thermische Geschwindigkeit des Kerns der Verteilung der Protonen davon unberührt bleibt, steigt $\beta_{\|, k}$ an, wodurch die Spiegel-Instabilität begünstigt wird. Das Auftreten der Spiegel-Instabilität ist auch mit überdurchschnittlichen Temperaturanisotropien verbunden. Die Ursache der Einbrüche des Betrages des magnetischen Feldes kann ich nicht benennen (s. dazu Zurbuchen et al. 2001). Die Bedeutung der Spiegel-Instabilität für die mittleren Eigenschaften des schnellen Sonnenwindes schätze ich wegen ihrer geringen Häufigkeit als vernachlässigbar ein. Für die Mittelwerte der in den ausgewerteten Sonnenwindströmungen gemessenen Parameter (s. Tabelle A.1) existiert die Spiegel-Instabilität nicht. Tsurutani et al. (1992) berichten, daß sie in von Ulysses im schnellen Sonnenwind aufgenommenen Magnetfelddaten die Moden dieser Instabilität hätten nachweisen können. Diese Messungen stammen aus einer Entfernung von 2.2 AE und im Gegensatz zu den Daten dieser Arbeit aus einer Phase maximaler solarer Aktivität. Mir sind keine weiteren Beobachtungen dieser Moden im Sonnenwind bekannt.

\subsection{Instabilitäten bei paralleler Ausbreitungsrichtung}

Bei paralleler Ausbreitungsrichtung besitzen zwei Instabilitäten, die durch die Temperaturanisotropie des Kerns der Geschwindigkeitsverteilung der Protonen verursachten werden, und eine vom Protonenstrahl ausgelöste Instabilität der magnetosonischen Moden 


\begin{tabular}{ccccc}
\hline \hline Parameter & Protonen-Kern & Protonen-Strahl & $\alpha$-Teilchen & Elektronen \\
\hline$n / n_{p}$ & 0.96 & 0.04 & 0.01 & 1.02 \\
$T_{\|} / T_{\|, k}$ & 1 & 1.55 & 11.8 & 0.75 \\
$T_{\perp} / T_{\|}$ & 3.5 & 2.3 & 1 & 1 \\
\hline \hline
\end{tabular}

Tabelle 6.2: Aus den auf Helios 2 am 14. April 1976 um 9h/5m/33s gemessenen Geschwindigkeitsverteilungen der Protonen, Elektronen und $\alpha$-Teilchen abgeleitete Plasmaparameter. Desweiteren sind $v_{A} / c=5.6 \times 10^{-4}, \beta_{\|, k}=0.29, \Delta v_{k, s}=1.05 v_{A}$, $\Delta v_{k, \alpha}=1.06 v_{A}$ und $T_{\alpha} / T_{p}=4.2$. Die genannten Parameter führen zu einer SpiegelInstabilität.

ihre maximale Anwachsrate. Die Temperaturanisotropie kann die auswärts laufenden ${ }^{4}$ Alfvén-Moden und einen Zweig von einwärts laufenden Zyklotron-Moden, der in der Abbildung 2.1b gestrichelt dargestellt ist, instabil werden lassen. Die möglicherweise instabilen magnetosonischen Moden laufen auswärts. Die direkte Berechnung der Anwachsraten dieser Instabilitäten beruht auf der Lösung der im Anhang hergeleiteten Dispersionsbeziehung D.21 der Zyklotronmoden, wie sie sich aus der Entwicklung der gemessenen Verteilungen der Protonen nach Gaußfunktionen (s. Kapitel 4) ergibt. Es wurde die im Abschnitt 6.2 diskutierte Vereinfachung der reduzierten Geschwindigkeitsverteilungen der Protonen vorgenommen. Als Resultat der Auswertung liegen die in der Abbildung 2.1b gezeigten Zyklotronzweige auf einem regulären Gitter mit $6 \times 10^{-3} \leq k_{\|} c / \omega_{p p} \leq 25$ und einer Auflösung von 400 Punkten vor ${ }^{5}$. Der große Wert $k_{\|} c / \omega_{p p}=25$ wurde im Hinblick auf die Untersuchung des Kapitels 7 gewählt. Dort werden diejenigen Dispersionszweige der Zyklotronmoden benötigt, die mit den Protonen im Kern der Geschwindigkeitsverteilung resonant sind. Die Resonanzgeschwindigkeiten dieser Moden erreichen mit ansteigendem Betrag der Wellenzahl das Zentrum des Kerns der Verteilung (s. Abbildungen 7.2a und 7.2b) und sollen sich ihm innerhalb des vom Gitter umfaßten Bereichs hinreichend annähern.

Die Ergebnisse der direkten Berechnung der Anwachsraten der Instabilitäten sind in den Abbildungen 6.12a-6.12f zusammengefaßt. Sie zeigen für jede der drei Instabilitätstypen das normierte Histogramm der beobachteten maximalen Anwachsraten und die Häufigkeiten der jeweiligen Instabilität in den ausgewerteten Sonnenwindströmungen, welche über die mittleren Entfernungen der Messungen aus diesen Strömungenzur Sonne aufgetragen sind. Im Zusammenhang mit der Untersuchung der Instabilitäten bei schräger Ausbreitungsrichtung wurden die Dispersionszweige unter Verwendung der im Abschnitt 6.1 beschriebenen Kern-Strahl-Approximation der Geschwindigkeitsverteilungen der Protonen berechnet. Die daraus folgenden Ergebnisse zu den Instabilitätstypen sind in den Abbildungen 6.12a-6.12f zum Vergleich hinzugefügt. Die Mittelwerte der maximalen Anwachsraten betragen $\gamma_{m} / \Omega_{p}=1.2 \times 10^{-2}, \gamma_{m} / \Omega_{p}=1.9 \times 10^{-2}$ und $\gamma_{m} / \Omega_{p}=$ $5.6 \times 10^{-3}$ für die Instabilitäten der auswärts laufenden Alfvén-Moden, der einwärts

\footnotetext{
${ }^{4}$ definiert über die Phasengeschwindigkeit der Moden

${ }^{5}$ Es wurde wie zuvor von der Symmetrie $k_{\|} \rightarrow-k_{\|}, \omega \rightarrow-\omega^{*}$ der Dispersionsbeziehung Gebrauch gemacht. Links-zirkular polarisierte Moden gehen dadurch in recht-zirkular polarisierte über und umgekehrt.
} 
laufenden Zyklotronmoden bzw. der magnetosonischen Moden. Unter Verwendung der Kern-Strahl-Approximation ergeben sich in der gleichen Reihenfolge die Mittelwerte $\gamma_{m} / \Omega_{p}=1.7 \times 10^{-2}, \gamma_{m} / \Omega_{p}=1.3 \times 10^{-2}$ bzw. $\gamma_{m} / \Omega_{p}=7.7 \times 10^{-3}$. Es fanden sich 9708 , 1273 und 2060 Instabilitäten der auswärts laufenden Alfvén-Moden, der einwärts laufenden Zyklotronmoden bzw. der magnetosonischen Moden. Unter Verwendung der Kern-Strahl-Approximation beträgt die Anzahl der Instabilitäten in dieser Reihenfolge 9741, 611 bzw. 766, wobei 10804 Datensätze ausgewertet wurden. Die Verwendung der Kern-Strahl-Approximation führt dazu, daß die instabilen auswärts laufenden Alfvén-Moden häufiger mit einer großen maximalen Anwachsrate auftreten. Die Gesamthäufigkeit dieser Instabilität bleibt allerdings unberührt. Die einwärts laufenden Zyklotronmoden sind für die Kern-Strahl-Approximation der Verteilung der Protonen seltener instabil. Das Histogramm ihrer Anwachsraten ändert sich kaum. Die Häufigkeit der magnetosonischen Instabilität fällt durch die Verwendung der Kern-Strahl-Approximation in allen Entfernungen zur Sonne deutlich geringer aus.

Die Anwachsraten einer instabilen Mode hängt aufgrund der beobachteten Größenordnung der maximalen Anwachsraten im wesentlichen von der Form der Geschwindigkeitsverteilungen der Protonen in der Nähe ihrer Resonanzgeschwindigkeit $v_{\text {res }}=(\omega-$ $\left.\Omega_{p}\right) / k_{\|}{ }^{6}$ ab (s. Gleichung 2.18). Die Resonanzgeschwindigkeiten der Moden maximaler Anwachsrate der von der Temperaturanisotropie des Kerns verursachten Instabilitäten befinden sich jeweils auf einer Seite des Kerns der Geschwindigkeitsverteilung der Protonen, wo die gemessenen Verteilungen durch große Zählraten des Instrumentes 1a festgelegt sind. Deshalb sollte die Entwicklung der Verteilung nach Gaußfunktionen zu besseren Ergebnissen als die Kern-Strahl-Approximation führen. Aus diesem Grund wird die aus dieser Darstellung berechnete Dispersion von Zyklotronmoden in den Untersuchungen des Kapitels 7 über Diffusionsplateaus in den beobachteten Verteilungen der Protonen verwendet. Die Mode maximaler Anwachsrate der magnetosonischen Instabilität hat ihre Resonanz im Protonenstrahl, von dem sie verursacht wird. Die Abbildungen 6.13a und $6.13 \mathrm{~b}$ verdeutlichen, daß es sich dabei nicht um den regulären Protonenstrahl handeln muß. In der Abbildung 6.13a sind zwei reduzierte Verteilungen nullter Ordnung der Protonen zu sehen. Beide ergeben sich aus der Entwicklung 4.1 einer auf Helios 2 gemessenen Geschwindigkeitsverteilung nach Gaußfunktionen und unterscheiden sich dadurch, daß in einem Fall die Beiträge von Kanälen des Instrumentes 1a zu der Entwicklung vernachlässigt wurden, die zu einem weiteren Strahl von Protonen mit sehr geringer Teilchendichte und vergleichsweise großer Geschwindigkeit führen. Dieser Strahl verursacht eine Instabilität der magnetosonischen Moden. Die Anwachsraten und die Resonanzgeschwindigkeiten dieser Moden sind in der Abbildung 6.13b in Abhängigkeit von der Wellenzahl für die Entwicklung 4.1 mit und ohne den zweiten Protonenstrahl dargestellt. Die magnetosonischen Moden sind nur instabil, wenn der Protonenstrahl beibehalten wird. Die Resonanzgeschwindigkeiten der instabilen Moden befinden sich innerhalb dieses Protonenstrahls. Solche Protonenstrahlen treten in ähnlicher Form in vielen Geschwindigkeitsverteilungen der Protonen auf und werden durch sehr kleine Zählraten, die nicht viel größer als Eins sind, erzeugt. Da die maximale Anwachsrate der magnetosonischen

\footnotetext{
${ }^{6}$ Die Symmetrie $k_{\|} \rightarrow-k_{\|}, \omega \rightarrow-\omega^{*}$ der Dispersionsbeziehung erlaubt es, sich auf Lösungen der Dispersionsbeziehung zu $D_{-}$einzuschränken. Davon wird in dieser Arbeit stets Gebrauch gemacht (s.a. Abbildungen 2.1a und 2.1b).
} 
(a)

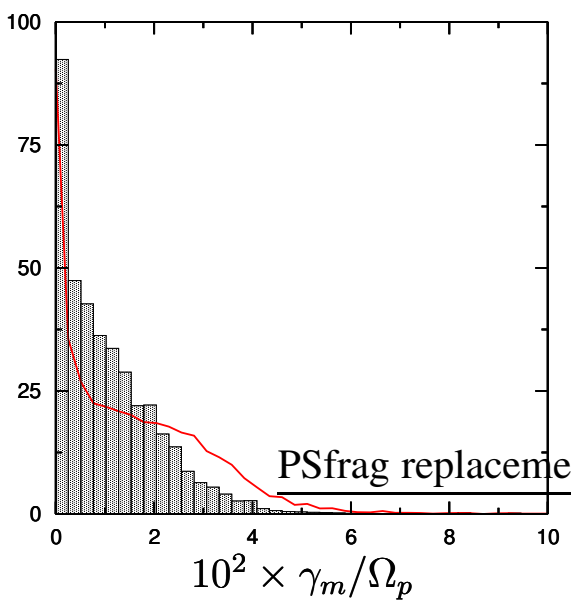

(c)

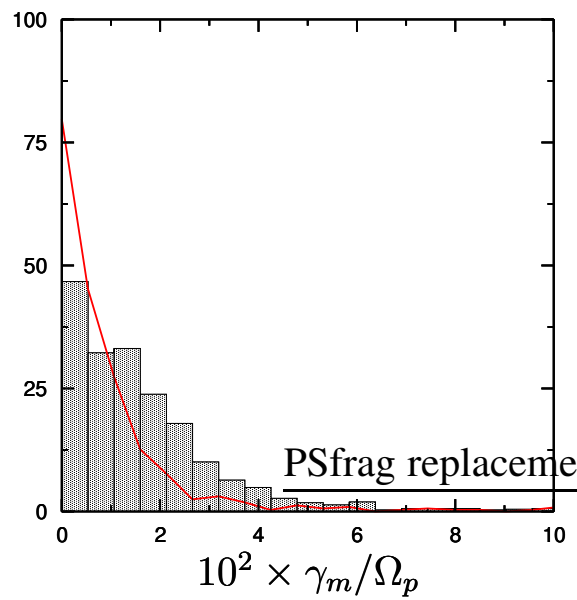

(e)

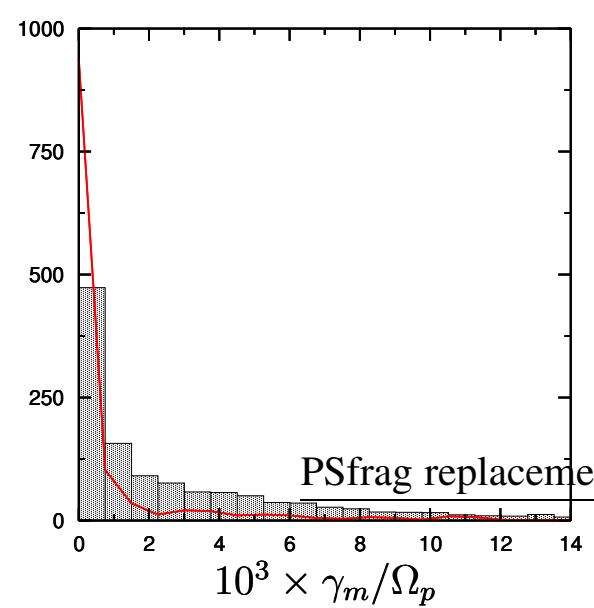

(b)

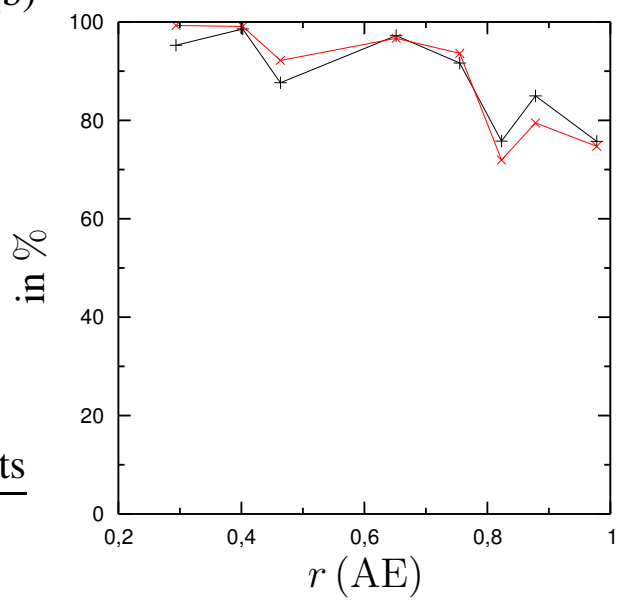

(d)

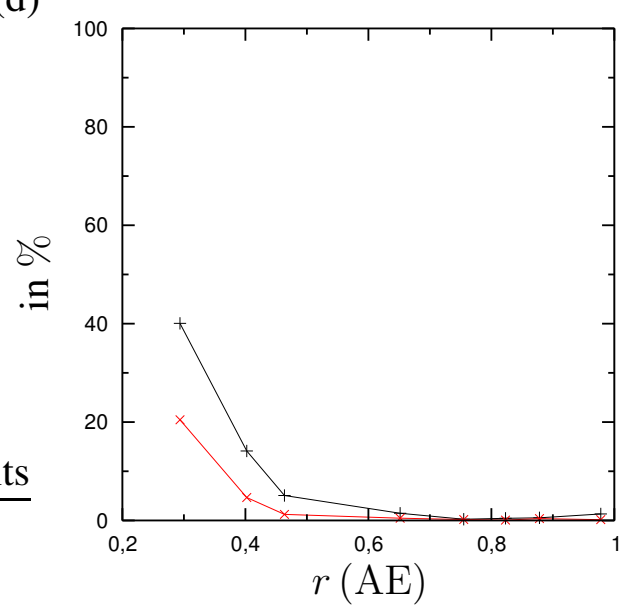

(f)

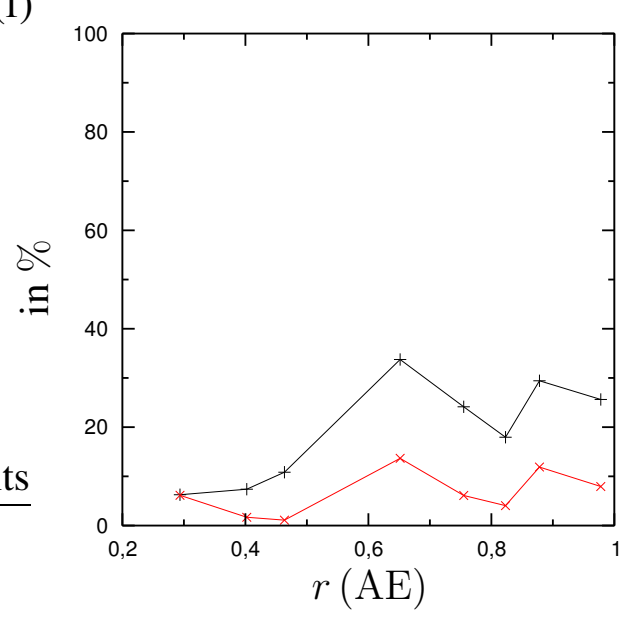

Abbildung 6.12: (a),(c) und (e) zeigen normierte Histogramme der maximalen Anwachsrate der auswärts laufenden Alfvén-Moden, der einwärts laufenden Zyklotronmoden (s. Text) bzw. der auswärts laufenden magnetosonischen Moden. (b),(d) und (f) zeigen die Häufigkeit dieser Instabilitäten in den ausgewählten Strömungen schnellen Sonnenwindes. Sie sind in abhängigkeit von der mittleren Entfernungen der Messungen in den Strömungen zur Sonnne aufgetragen. Die roten Kurven zeigen jeweils die entsprechenden Ergebnisse unter Verwendung der Kern-Strahl-Approximation der Verteilung der Protonen. 
(a)

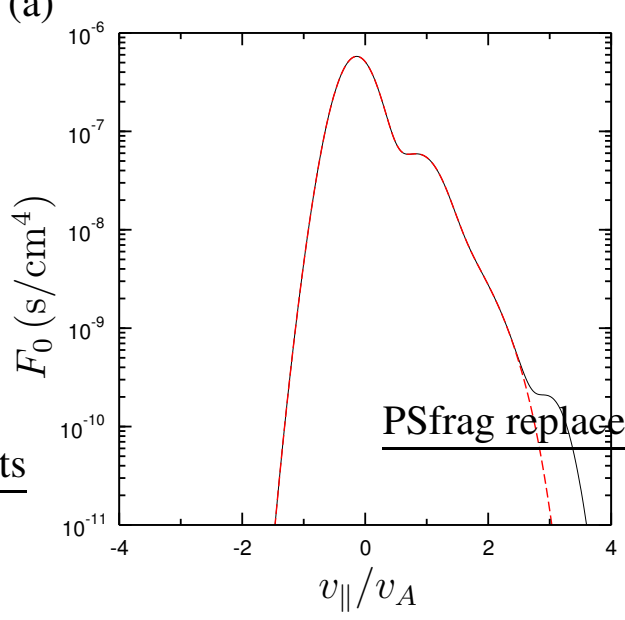

(b)

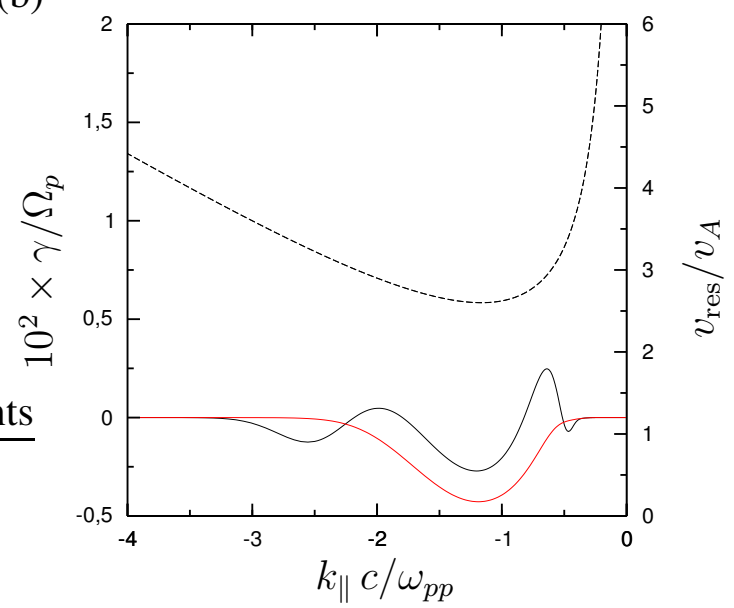

Abbildung 6.13: (a) Reduzierte Verteilungen nullter Ordnung der auf Helios 2 am 16. März 1976 um 23h/33m/12s gemessenen Geschwindigkeitsverteilung der Protonen mit (schwarz) und ohne (rot) den Beitrag derjenigen Kanäle des Instrumentes 1a zur Darstellung 4.1 der Verteilung, die einen irregulären Protonenstrahl verursachen. (b) Anwachsrate der magnetosonischen Moden mit (schwarz) und ohne (rot) Berücksichtigung dieser Beiträge zur Verteilung der Protonen. Die Resonanzgeschwindigkeiten der magnetosonischen Moden sind durch die gestrichelte Kurve dargestellt.

Instabilität bei paralleler Ausbreitungsrichtung mit der Geschwindigkeit des ursächlichen Protonenstrahls stark ansteigt (vgl. Abbildung 2.4), können diese Strahlen trotz ihrer sehr geringen Teilchendichte eine verhältnismäßig große Anwachsrate verursachen. Durch die Kern-Strahl-Approximation der Verteilungen der Protonen (s. Abschnitt 6.1) werden sie nicht berücksichtigt, weshalb sich auf diese Weise deutlich geringere Häufigkeiten und maximale Anwachsraten der magnetonischen Instabilität ergeben haben (s. Abbildungen 6.12e und 6.12f). Der Mittelwert der maximalen Anwachsrate der magnetosonischen Instabilität beträgt $\gamma_{m} / \Omega_{p}=8.5 \times 10^{-4}$, wenn die nicht im Histogramm 6.12e sichtbaren 74 Fälle mit $\gamma_{m} / \Omega_{p}>1.4 \times 10^{-2}$ vernachlässigt werden. Diese Einzelfälle sind wie für die Strahl-Instabilitäten bei schräger Ausbreitung mit kurzzeitigen Einbrüchen des Betrages des magnetischen Feldes und somit sehr kleinen Werten der Alfvén-Geschwindigkeit verbunden $\left(v_{A} / c \lesssim 2 \times 10^{-4}\right)$. Die auswärts laufenden magnetosonischen Moden sind bei paralleler Ausbreitungsrichtung mit einer Gesamthäufigkeit von $7 \%$ selten instabil. Diese Strahl-Instabilität kann in Anbetracht des Mittelwertes ihrer maximalen Anwachsrate genauso wie die Strahl-Instabilitäten bei schräger Ausbreitungsrichtung (s. Abschnitt 6.3.1) allenfalls marginal instabil sein. Im schnellen Sonnenwind existieren somit bis zu der Entfernung von $1 \mathrm{AE}$ zur Sonne keine starken Strahl-Instabilitäten. Bisher wurde aufgrund der Arbeit von Marsch und Livi (1987) das Gegenteil angenommen (s. z.B. Daughton und Gary 1998, Daughton et al. 1999).

Die Ergebnisse zeigen, daß die auswärts laufenden Alfvén-Moden, deren Instabilität von der Temperaturanisotropie des Kerns der Verteilung der Protonen verursacht wird, im mittleren Zustand des schnellen Sonnenwindes nicht bloß marginal instabil sein können. Ihre maximalen Anwachsraten übersteigen die aufgrund der Diskussion des Abschnittes 2.2.3 zu erwartenden Werte um mehr als zwei Größenordnungen. Diese Instabilität betrifft 
mit einer Gesamthäufigkeit von $90 \%$ nahezu jede gemessene Geschwindigkeitsverteilung und diese Häufigkeit wird selbst in der Entfernung von 0.98 AE kaum unterschritten. Die von der Temperaturanisotropie ausgelöste Instabilität der einwärts laufenden Zyklotronmoden tritt nur in der Nähe des Perihels von Helios mit bedeutender Häufigkeit auf. Die dort beobachteten Anwachsraten dürfen ebenfalls nicht als marginal bezeichnet werden. Die quasi-lineare Näherung der Welle-Teilchen-Wechselwirkung verlangt, daß die Instabilitäten im Verlauf der Expansion des schnellen Sonnenwindes auf der durch die linearen Anwachsraten vorgegebenen Zeitskala von $T \approx 10^{2} \mathrm{~s}$ bzw. einer räumlichen Skala von $L=V T \approx 10^{-3} \mathrm{AE}$ relaxieren, wobei von der typischen Größenordnung $\Omega_{p}=1 \mathrm{~s}^{-1}$ der Zyklotronfrequenz der Protonen und einem Höchstwert $V \approx 10^{3} \mathrm{~km} \mathrm{~s}^{-1}$ der Expansionsgeschwindigkeit des Windes ausgegangen wurde. Demnach sollten sie sich nicht beständig über zwischen $0.3 \mathrm{AE}$ und $1 \mathrm{AE}$ beobachten lassen.

Die Abbildungen 6.14a-6.14h sollen Aufschluß geben, ob eine Relaxation der Instabilitäten im Verlauf der Expansion des schnellen Sonnenwindes stattfindet. Dafür zeigen sie Stabilitätsgrenzen der von der Temperaturanisotropie des Kerns der Verteilung der Protonen ausgelösten Instabilitäten in der von dem Plasmabeta und Temperaturanisotropie des Kerns gebildeten Parameterebene. Die gemessenen Werten dieser Parameter sind in Form von Streudiagrammen ebenfalls in den Abbildungen, die zwischen den ausgewerteten Strömungen des schnellen Sonnenwindes und der Entfernung zur Sonne unterscheiden, zu sehen. Die Stabilitätgrenzen sind durch den Wert $\gamma_{m} / \Omega_{p}=10^{-3}$ der maximalen Anwachsrate definiert und ihnen liegen die in der Tabelle A.1 zusammengestellten Mittelwerte der Plasmaparameter in ausgewerteten Strömungen zugrunde. Es ist zu erkennen, daß die Menge der Parameterwerte sich mit wachsender Entfernung zur Sonne auf die Stabilitätsgrenze der auswärts laufenden Alfvén-Moden zubewegen und sich in der Nähe von $1 \mathrm{AE}$ entlang der Stabilitätsgrenze verteilen. In geringerer Entfernung zur Sonne befinden sich im Einklang mit den Resultaten der direkten Berechnung der Anwachsraten der Instabilität der Alfvén-Moden praktisch alle Wertepaare oberhalb der Stabilitätgrenze. Im Perihel von Helios 2 reicht die Größe der Temperaturanisotropie noch aus, um die Instabilität der einwärts laufenden Zyklotronmoden zu verursachen. Im Verlauf der Relaxation der linearen Instabilität der auswärts laufenden Alfvén-Moden nehmen der Mittelwert und die Varianz des Plasmabetas des Kerns der Verteilung der Protonen zu (vgl. Abbildung A.3a). Die Temperaturanisotropie des Kerns nimmt weniger stark ab, als es bei einer adiabatischen Expansion des Windes zu erwarten ist (vgl. Abbildung A.3e). Eine Regression führt zu den Potenzgesetzen $T_{\perp, k} / T_{\|, k}=1.62(r / \mathrm{AE})^{-0.49}$ und $\beta_{\|, k}=0.4(\mathrm{r} / \mathrm{AE})^{0.91}$ zwischen den Parametern und der Entfernung $r$ zur Sonne. Marsch et al. (2004) beobachteten im schnellen Sonnenwind, ohne die Entfernung zur Sonne zu berücksichtigen, eine direkte Korrelation zwischen dem Plasmabeta und der Temperaturanisotropie des Kerns $\left(T_{\perp, k} / T_{\|, k} \approx 1.16 \beta_{\|, k}^{-0.55}\right)$, die sich aus dieser Entfernungsabhängigkeit der Parameter im Zusammenhang mit der Relaxation erklärt.

Die Ergebnisse dieses Abschnittes zeigen, daß die nicht-lineare Wechselwirkung der mit den Protonen resonanten auswärts laufenden Alfvén-Moden im Bereich der Zyklotronfrequenzen der Ionen nicht vernachlässigt werden darf. Die nicht-lineare Dynamik der Wellenmoden mit wesentlich kleiner Frequenz und Wellenzahl, d.h. im Regime der MHD kann seit der Arbeit von Belcher und Davis (1971) nicht bezweifelt werden. Sie beobachteten im schnellen Sonnenwind Alfvén-Wellen, deren Energiedichte mit derjenigen 
(a)

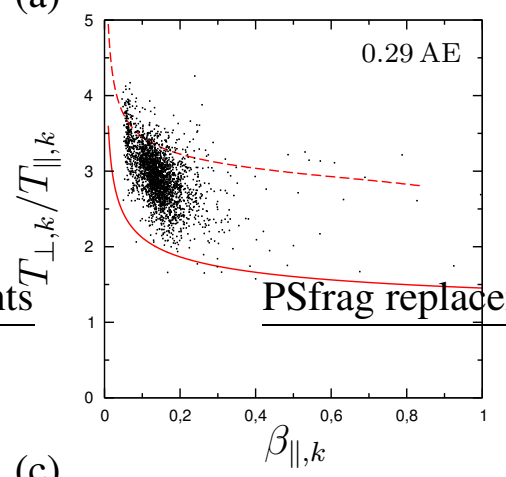

(c)

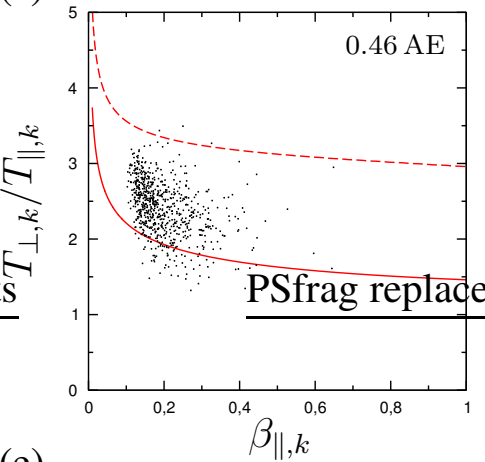

(e)

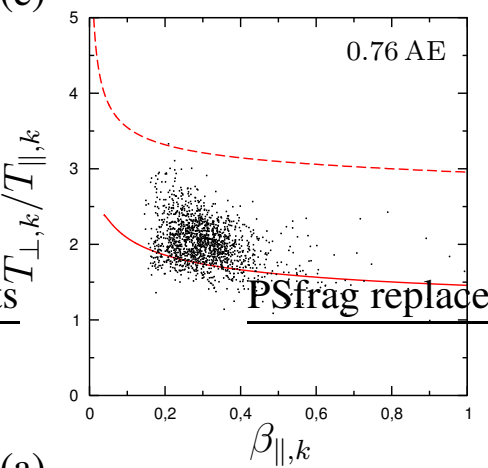

(a)

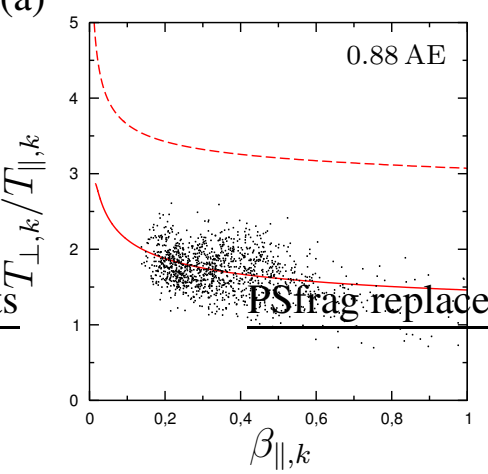

(b)

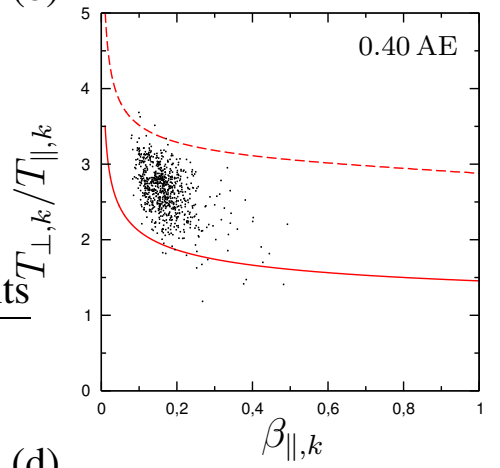

(d)

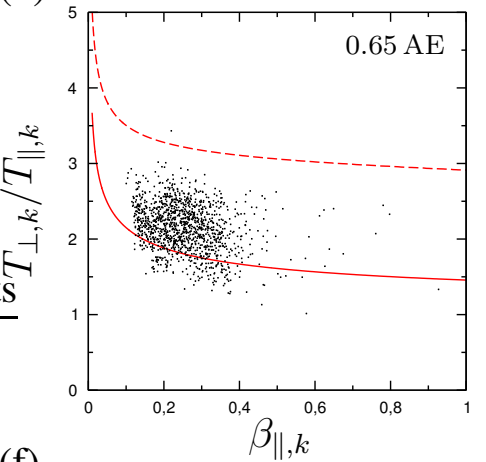

(f)

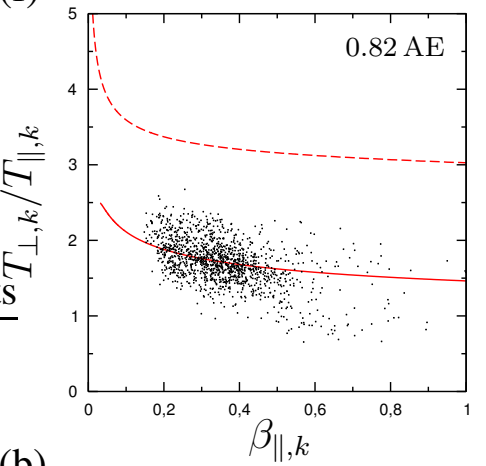

(b)

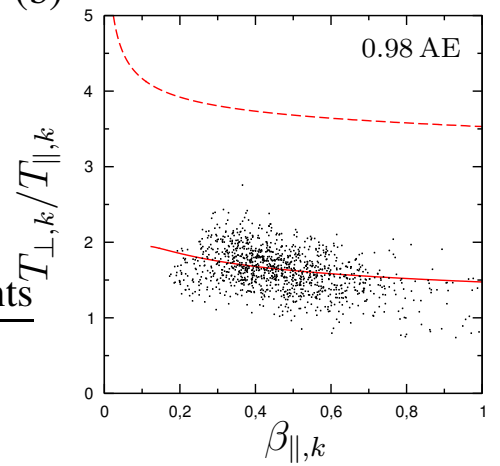

Abbildung 6.14: (a)-(f) Stabilitätsgrenzen der von der Temperaturanisotropie des Kerns der Verteilung der Protonen ausgelösten Instabilitäten in der zusammen mit dem Plasmabeta des Kerns gebildeten Parameterebene (auswärts laufende Alfvén-Moden ( - ) und einwärts laufende Zyklotronmoden (---)) sowie Streudiagramme der gemessenen Werte dieser Parameter. Die Teilabbildungen unterscheiden nach den ausgewerteten Strömungen des schnellen Sonnenwindes (s. Tabelle 3.1). Den Stabilitätsgrenzen liegen jeweils die Mittelwerte der Plasmaparameter aus diesen Strömungen (s. Tabelle A.1) und der Wert $\gamma_{m} / \Omega_{p}=10^{-3}$ der maximalen Anwachsrate zugrunde. 
des mittleren magnetischen Feldes nahezu vergleichbar ist. Die auswärts laufenden und resonanten Alfvén-Wellen sind für das Entstehen der Temperaturanisotropie des Kerns der Verteilung der Protonen und der nicht-adiabatischen Abnahme der Anisotropie mit der Entfernung zur Sonne verantwortlich (s. Kapitel 7). Das Überschreiten der Grenze absoluter Stabilität, was vor dem Perihel von Helios und unter Umständen noch innerhalb der Korona im Zusammenhang mit dem Entstehen der Temperaturanisotropie geschieht, ist mit der quasi-linearen Näherung genauso wie das Verweilen im Bereich linearer Instabilität zwischen 0.3 AE und $1 \mathrm{AE}$ unvereinbar. Die nicht-lineare Welle-TeilchenWechselwirkung beinhaltet Modenkopplungen und die nicht-lineare Dämpfung und Instabilitäten von Wellenmoden (s.z.B. Sagdeev und Galeev 1969, Davidson 1972, Galeev und Sagdeev 1983b,c). Letztere sind mit Resonanzen zwischen mehreren Wellenmoden und den Teilchen verbunden, z.B. $\omega^{\prime}+\omega^{\prime \prime}-\left(k_{\|}^{\prime}+k_{\|}^{\prime \prime}\right) v_{\|}+n \Omega_{s}=0$ in der untersten nicht-linearen Ordnung, über die sie zusätzlich zu den linearen Resonanzen (s. Gleichungen 2.40-2.43) mit den Teilchen wechselwirken. Es ist denkbar, daß die lineare Instabilität der auswärts laufenden Alfvén-Moden durch eine nicht-lineare Dämpfung, an der mehrere Wellenmoden beteiligt sein müssen, ausgeglichen wird. Modenkopplungen können ebenfalls zur Stabilisierung der linearen instabilen Moden beitragen. Es muß ggf. herausgefunden werden, welche Moden an einer nicht-linearen Dämpfung oder an einer Modenkopplung der Alfvén-Moden beteiligt sind und welchen Einfluß diese Moden auf die Geschwindigkeitsverteilung besitzen. Möglicherweise steht eine Modenkopplung der instabilen Alfvén-Moden in einem Zusammenhang mit einer direkten Kaskade, die Energie von den groß- zu den kleinskaligen Fluktuationen befördern soll (s. z.B. Tu et al. 1984), deren Bestandteil die mit den Protonen resonanten Alfvén-Moden sind. 



\section{Untersuchung von Diffusionsplateaus im schnellen Sonnenwind}

Im schnellen Sonnenwind zeigen die Geschwindigkeitsverteilungen der Protonen in ihrem Kern eine Temperaturanisotropie. Die Temperatur des Kerns senkrecht zum mittleren magnetischen Feld ist größer ist als seine Temperatur parallel dazu. Aus diesem Grund muß geschlußfolgert werden, daß die Protonen im Kern der Verteilung einer Heizung unterliegen. Für diesen Prozeß werden Alfvén-Wellen im Bereich der Zyklotronfrequenzen der Ionen verantwortlich gemacht (Marsch et al. 1982a, Marsch und Tu 2001, Tu und Marsch 2002, Marsch et al. 2004). Von ihnen wird angenommen, daß sie ihren Ursprung auf der Sonne besitzen und entlang des magnetischen Feldes auswärts laufen. Die Alfvén-Wellen sollen mit den Protonen gemäß der quasi-linearen Näherung wechselwirken, wodurch die beobachteten Temperaturanisotropien entstehen können (Arunasalam 1976). Im Abschnitt 2.3 dieser Arbeit wurde gezeigt, daß die Protonen durch diese Wechselwirkung im Geschwindigkeitsraum diffundieren. Daraus folgt unter geeigneten Umständen die Ausbildung von Diffusionsplateaus in ihren Geschwindigkeitsverteilungen. Es war eine Zielsetzung meiner Arbeit, diese Vorhersage im schnellen Sonnenwind zu untersuchen. Aus der Beobachtung von Diffusionsplateaus im schnellen Sonnenwind folgt somit die Existenz der Alfvén-Wellen im Bereich der Zyklotronfrequenzen der Ionen. Im Sonnenwind sind Alfvén-Wellen bisher nur bei Frequenzen nachgewiesen, die deutlich kleiner als die Zyklotronfrequenz der Protonen sind (Belcher und Davis 1971). Außerdem beweist eine solche Beobachtung, daß die zur quasi-linearen Diffusion führenden Annahmen im schnellen Sonnenwind weitgehend gültig sind. Die Vorstellung von der schwachen Turbulenz des schnellen Sonnenwindes muß zutreffen und es gilt eine Skalentrennung zwischen den Fluktuationen und dem Hintergrund der mittleren Felder. Im Abschnitt 7.1 beschreibe ich die Ergebnisse der Untersuchung dieser Arbeit. Die ausgewerteten Daten werden im Abschnitt 3.2 dargestellt. Die von Tu et al. (2002) aufgeworfene Frage, ob die quasi-lineare Diffusion ebenfalls für die Form des im schnellen Sonnenwind beobachteten Protonenstrahls verantwortlich ist, soll im Abschnitt 7.2 diskutiert werden. 


\subsection{Diffusionsplateaus im Kern der Verteilung der Pro- tonen}

Die Erwartung, daß sich Diffusionsplateaus in den Geschwindigkeitsverteilungen der Protonen des schnellen Sonnenwindes beobachten lassen, ist durch eine Abschätzung der Größenordnung der quasi-linearen Diffusion begründet. Die typische Zeitskala $\tau_{D}$, auf der sich die Geschwindigkeitsverteilung der Protonen durch die Diffusion ändert, beträgt $\tau_{D} \approx V^{2} / D$. Dabei sind $D$ und $V$ die typische Größenordnung der Diffusionskoeffizienten 2.48-2.50 bzw. die typische Skala der Gradienten der Geschwindigkeitsverteilung. Die Diffusionskoeffizienten besitzen die übersichtliche Form

$$
D_{a b}=\frac{\pi}{2}\left(\frac{e_{p}}{m_{p}}\right)^{2} \int \frac{d k_{\|}}{\sqrt{2 \pi}} \delta\left(\omega_{k_{\|}}-k_{\|} v_{\|}-\Omega_{p}\right)\left(\frac{\omega_{k_{\|}}}{c k_{\|}}\right)^{2} P_{B}\left(k_{\|}\right) \vartheta_{a} \vartheta_{b}
$$

mit $\vartheta_{\|}=v_{\perp} k_{\|} / \omega_{k_{\|}}$und $\vartheta_{\perp}=1-v_{\|} k_{\|} / \omega_{k_{\|}}$, wobei ausgehend von 2.48-2.50 das Spektrum der Fluktuationen des elektrischen Feldes über das Faraday-Gesetz durch dasjenige des magnetischen Feldes ausgetauscht wurde. Daraus folgt unmittelbar die typische Größenordnung der Koeffizienten

$$
D \approx \frac{\pi}{2}\left(\frac{e_{p}}{m_{p}}\right)^{2}\left(\frac{v_{A}}{c}\right)^{2} \frac{P_{B}}{\sqrt{2 \pi} v_{A}}
$$

mit einem typischen Wert $P_{B}$ für das Fluktuationsspektrum im Bereich resonanter Wellenzahlen. Die Koeffizienten $\vartheta_{a}$ tragen im Kern der Geschwindigkeitsverteilung mit der Größenordnung $1 \mathrm{zu}$ den Diffusionskoeffizienten bei und alle Wellengeschwindigkeiten dürfen durch die Alfvén-Geschwindigkeit abgeschätzt werden. Der typische Wert für $P_{B}$ muß aus Messungen bei wesentlich kleineren Frequenzen bzw. Wellenzahlen durch Extrapolation abgeleitet werden. Die von Denskat und Neubauer (1983) erarbeiteten Spektren führen zu groben Schätzwerten von $P_{B} / v_{A} \approx 10^{-10} \mathrm{G}^{2} \mathrm{~s}$ und $P_{B} / v_{A} \approx 10^{-11} \mathrm{G}^{2} \mathrm{~s}$ in Entfernungen von 0.3 AE bzw. 1 AE zur Sonne. Daraus ergibt sich die typische Größenordnung der Diffusionskoeffizienten zu

$$
D \approx 10^{2} \mathrm{~km}^{2} / \mathrm{s}^{3}, \text { bzw. } D \approx 1 \mathrm{~km}^{2} / \mathrm{s}^{3}
$$

in diesen Entfernungen. Die typische Zeitskala der Diffusion lautet somit

$$
\tau_{D} \approx 10^{2} \mathrm{~s} \text {, bzw. } \tau_{D} \approx 10^{3} \mathrm{~s} .
$$

Dafür wurde die typische Skala der Gradienten der Geschwindigkeitsverteilung durch die Alfvén-Geschwindigkeit $v_{A}$ abgeschätzt, die bis $1 \mathrm{AE}$ größer als die thermische Geschwindigkeit der Protonen ist. Die Expansionszeitskala des Sonnenwindes, $\tau_{E} \approx r / 2 v$, beträgt im Vergleich zu $\tau_{D}$ bereits $\tau_{E} \approx 10^{4} \mathrm{~s}$ bzw. $\tau_{E} \approx 10^{5} \mathrm{~s}$. Die Diffusion dominiert über alle anderen Terme in der Bewegungsgleichung der Geschwindigkeitsverteilung. Eine stationäre Verteilung muß bis auf geringe Abweichungen Diffusionsplateaus ausgebildet haben.

Die Arbeiten von Marsch und Tu (2001) und Tu und Marsch (2002) haben erste Hinweise ergeben, daß sich Diffusionsplateaus tatsächlich in den Geschwindigkeitsverteilung der 
(a)

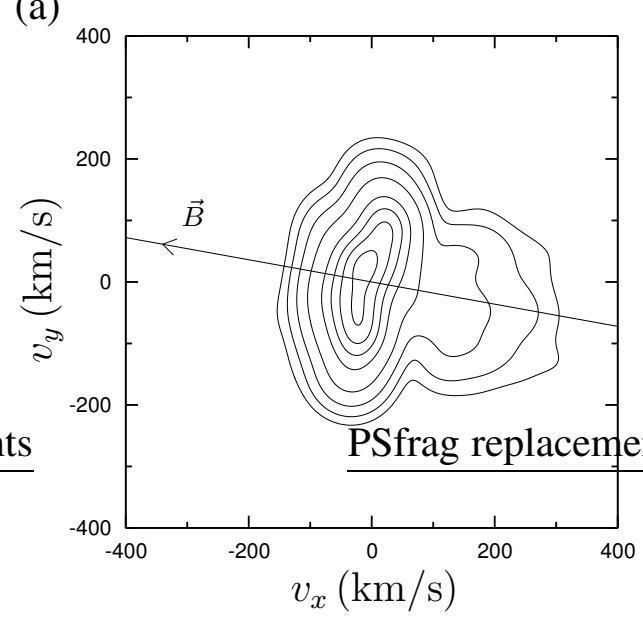

(b)

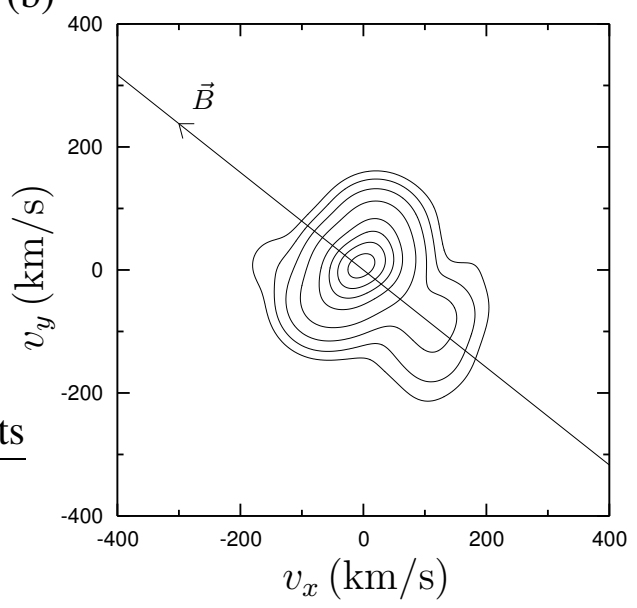

Abbildung 7.1: Höhenlinien der auf Helios 2 (a) am 14. April 1976 um 10h/46m/8s und (b) am 16. März um 4h/24m/52s gemessenen Geschwindigkeitsverteilung der Protonen in der im Kapitel 4 eingeführten Darstellung. Die Höhenlinien entsprechen den Anteilen 0.9, $0.7,0.5,0.3,0.1,0.03,0.01$ und 0.003 des Maximums der Verteilungen und wurden in der durch die mittlere Geschwindigkeit der Protonen und das magnetischen Feld definierten Ebene berechnet. Die Abszisse zeigt in die Richtung der mittleren Geschwindigkeit und der Ursprung stimmt mit ihr überein. Wichtige Parameter der Verteilungen sind in der Tabelle 7.1 zusammengefaßt.

Protonen beobachten lassen. Sie betrachteten einige wenige exemplarische Verteilungen und fanden durch einen graphischen Vergleich eine gute Übereinstimmung zwischen den Höhenlinien der Verteilungen und den theoretischen Diffusionsplateaus. Dafür mußten sie eine einfache und nicht von der Form der gemessenen Verteilungen abhängige Dispersionsbeziehung der Alfvén-Wellen vorgeben. Diese Vereinfachung wird in dieser Arbeit aufgegeben. Bevor ich auf die Auswertung der im Abschnitt 3.2 genannten Menge von Daten eingehe, möchte ich die in der Abbildung 7.1 gezeigten Geschwindigkeitsverteilungen der Protonen diskutieren. Sie wurden auf Helios 2 in einer Entfernung von 0.29 AE bzw. 0.66 AE gemessen. Diesen Darstellungen liegt genauso wie allen Ergebnissen dieses Abschnittes das im Kapitel 4 eingeführte Modell der Verteilungen zugrunde. Die entsprechende Dispersionsbeziehung (s. Anhang D) wurde unter der im Abschnitt 6.2 erläuterten Einschränkung mit der im Kapitel 5 beschriebenen Methode gelöst. Die Abbildungen 7.2a und 7.2b zeigen die Dispersionszweige der mit den Protonen im Kern der Verteilungen resonanten Wellenmoden und ihre Resonanzgeschwindigkeiten (vgl. Abbildung 2.6a). Die Wellenmoden bei positiven Wellenzahlen $k_{\|}$sind Alfvén-Moden. Sie breiten sich in Richtung des Protonenstrahls, d.h. auswärts aus. Die Resonanzgeschwindigkeit der Zyklotronmoden bei negativen Wellenzahlen $k_{\|}$ist beidseitig beschränkt. Sie können nicht mit den Protonen im Strahl in Resonanz treten. Die zu diesen Moden gehörenden Diffusionsplateaus liegen auf der dem Strahl zugewandten Seite des Kerns der Verteilungen, während die zu den auswärts laufenden Alfvén-Moden gehörenden Diffusionsplateaus auf der gegenüberliegenden Seite liegen (s. Abschnitt 2.3). Diese Topologie der Dispersionszweige ist repräsentativ für aller in dieser Arbeit ausgewerteten Verteilungen. In den Abbildung 7.2c und 7.2d sind Höhenlinien der um das mittlere magnetische Feld 
(a)

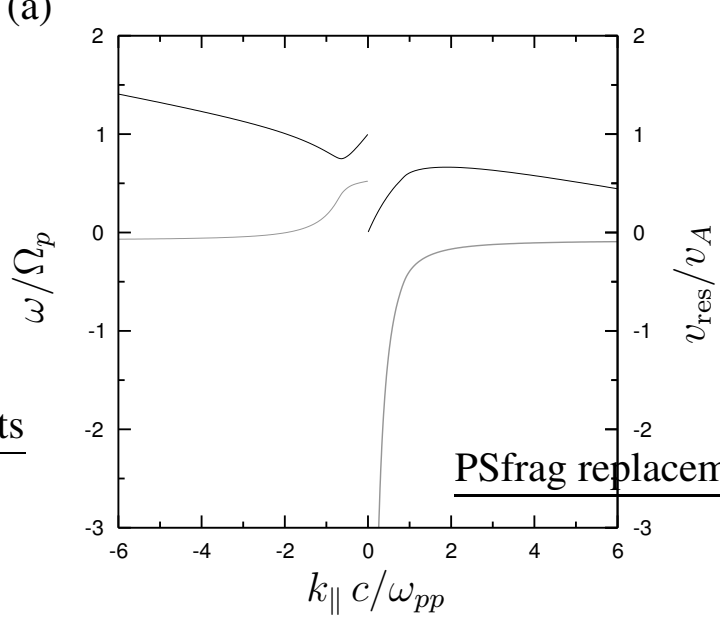

(c)

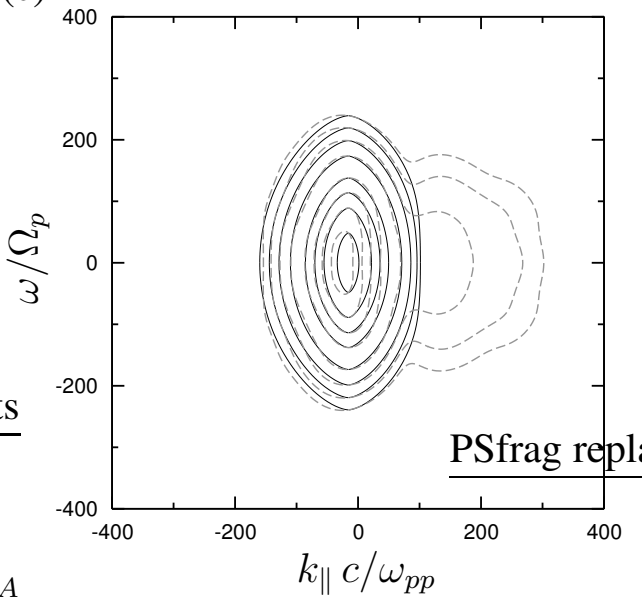

(b)

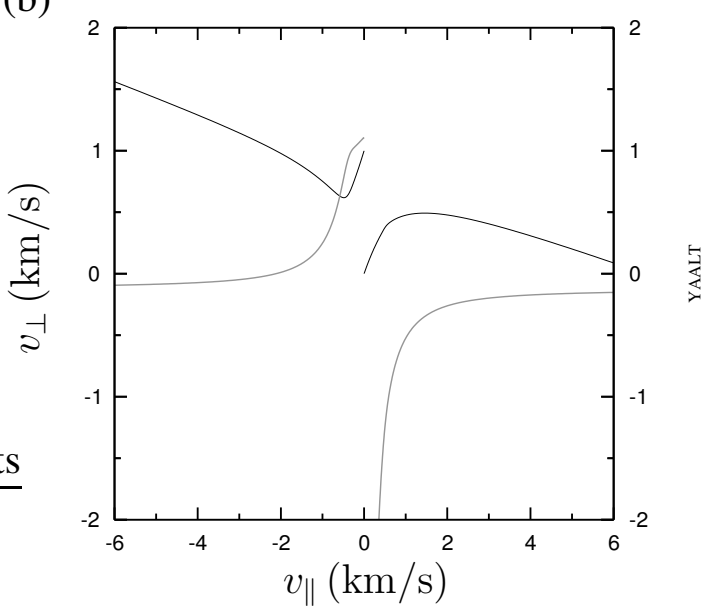

(d)

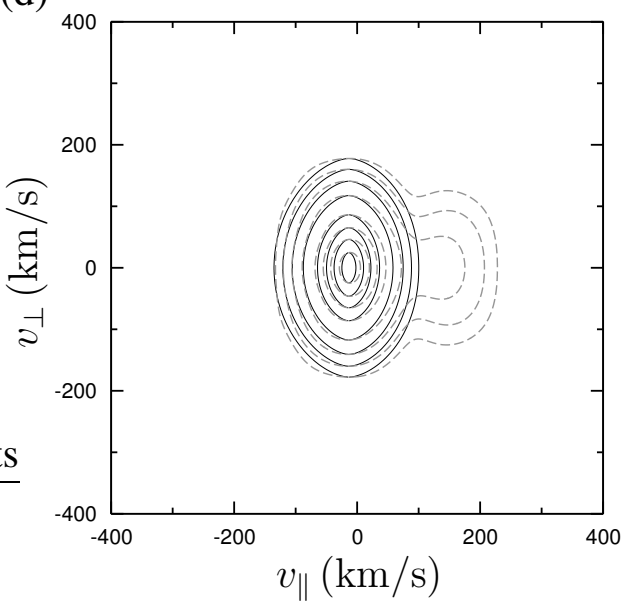

Abbildung 7.2: Die Abbildungen (a) und (b) zeigen die Dispersionszweige (schwarz) der Moden, die mit den Protonen im Kern der Geschwindigkeitsverteilung resonant sind. Ihnen liegen die auf Helios 2 (a) am 14. April 1976 um 10h/46m/8s bzw. (b) am 16. März um $4 \mathrm{~h} / 24 \mathrm{~m} / 52 \mathrm{~s}$ gemessenen Geschwindigkeitsverteilungen zugrunde. Die Resonanzgeschwindigkeit $v_{\text {res }}=\left(\omega-\Omega_{p}\right) / k_{\|}$dieser Moden wird durch die grauen Kurven dargestellt. In den Abbildungen (c) und (d) sind Höhenlinien (grau) der um das mittlere magnetische Feld symmetrisierten Verteilungen zu sehen. Die Höhenlinien entsprechen den Anteilen am Maximum der Verteilungen, wie sie in den Abbildungen 7.1a und 7.1b verwendet wurden. Den Höhenlinien sind die Diffusionsplateaus (schwarz) überlagert, die dieselben $v_{\perp}$-Achsenabschnitten im Zentrum des Kerns besitzen. Die Abbildungen (c) und (d) gehören zu den Abbildungen (a) bzw. (b).

symmetrisierten Verteilungen dargestellt. Ihnen sind die Diffusionsplateaus, die dieselben $v_{\perp}$-Achsenabschnitte im Zentrum des Kerns besitzen, überlagert (vgl. Abbildung 2.6b). Der Ursprung dieser Abbildungen stimmt mit der Geschwindigkeit des Massenschwerpunktes überein. Die Darstellung der Dispersionszweige bezieht sich ebenfalls auf dieses Ruhesystem des Plasmas. Die Höhenlinien der Verteilungen stimmen mit den theoretischen Diffusionsplateaus insbesondere auf der dem Strahl abgewandten Seite des Kerns gut überein. Die Ergebnisse von Marsch und Tu (2001) und Tu und Marsch (2002) werden 
damit auch ohne die von ihnen gemachten Vereinfachungen bestätigt.

Die Auswertung der insgesamt 10804 Geschwindigkeitsverteilungen (s. Abschnitt 3.2) auf ihre Abweichung von den theoretischen Diffusionsplateaus erfordert es, ein quantitatives $\mathrm{Ma}$ für diese Abweichung aufzustellen. Eine Möglichkeit, dieses Maß zu definieren, besteht darin, die Anisotropie von theoretischen Diffusionsplateaus und Höhenlinien zu vergleichen (Tu und Marsch 2002). Letztere könnte als scheinbare Anisotropie der Verteilung bezeichnet werden. Die Anisotropie einer Kurve ist dabei über $A=\left(V_{\perp} / V_{\|}\right)^{2}$ durch ihre Achsenabschnitte $V_{\perp}$ und $V_{\|}$definiert. Tu und Marsch (2002) gaben den Achsenabschnitt $V_{\|}$dabei so vor, daß die Verteilung dort den Bruchteil 0.3 ihres Maximums beträgt. Ich möchte den Unterschied zwischen den Anisotropien von Diffusionsplateaus und Höhenlinien nicht als Maß für die Abweichung von den Diffusionsplateaus verwenden, da das Ergebnis eines Vergleiches der Anisotropien von der Wahl eines Achsenabschnittes $V_{\|}$abhängt. Ich möchte auch nicht die Richtungsableitung der Verteilungen entlang der theoretischen Diffusionsplateaus betrachten. Es böte sich z.B. an, die Norm

$$
\int d v_{\|} d v_{\perp} v_{\perp}\left|\vartheta_{\|} \frac{\partial f}{\partial v_{\|}}+\vartheta_{\perp} \frac{\partial f}{\partial v_{\perp}}\right|^{2}
$$

als Maß für die Abweichung einer Verteilung von den Plateaus zu verwenden. Die Funktion $\epsilon \sin \omega t$ variiert mit der kleinen Amplitude $\epsilon$ nur schwach um Null. Durch die geeignete Wahl der Frequenz $\omega$ wird das Maximum ihrer Ableitung aber beliebig groß. Dementsprechend kann das Integral 7.5 groß sein, obwohl die Werte der Verteilungsfunktion $f$ entlang der Diffisionsplateaus nur schwach variieren. Schwankungen um die theoretischen Plateaus sind durch die in dieser Arbeit verwendete Darstellung der Geschwindigkeitsverteilungen nicht zu vermeiden. Ich beabsichtige, die Varianz der Werte einer Verteilung auf den theoretischen Diffusionsplateaus als Maß für die Abweichung der Verteilung von diesen Plateaus zu verwenden. Die Diffusionsplateaus sind die Höhenlinien der Funktion

$$
P\left(v_{\|}, v_{\perp}\right)=v_{\|}^{2}+v_{\perp}^{2}-2 \int^{v_{\|}} d v_{\|}^{\prime} \frac{\omega_{k_{\|}}}{k_{\|}}\left(v_{\|}^{\prime}\right)
$$

wobei die Phasengeschwindigkeit über die Resonanzbedingung eine Funktion der Geschwindigkeit $v_{\|}$ist. Diese Funktion erlaubt es, neue Koordinaten $P, \mu=\left(v_{\|}-v_{\|, 0}\right) / P$ einzuführen. Mit $v_{\|, 0}$ sei die Geschwindigkeit im Zentrum des Kerns der Verteilung bezeichnet. Das Mittel einer Geschwindigkeitsverteilung auf den Diffusionsplateaus ist in diesen Koordinaten durch

$$
\begin{aligned}
\langle f\rangle_{P}(P) & =\frac{1}{\Delta \mu(P)} \int_{\mu_{0}}^{\mu_{e}(P)} d \mu f(P, \mu) \\
& =\frac{1}{\Delta v_{\|}(P)} \int_{v_{\|, 0}}^{v_{\|, e}(P)} d v_{\|} f\left(v_{\|}, v_{\perp}\left(P, v_{\|}\right)\right)
\end{aligned}
$$

definiert, wobei $\Delta \mu(P)=\mu_{e}(P)-\mu_{e}(P), \Delta v_{\|}(P)=v_{\|, e}(P)-v_{\|, 0}$ und die Verteilungsfunktion $f$ in der Gleichung 7.7 durch $P$ und $\mu$ auszudrücken ist. Es werden die Symbole 


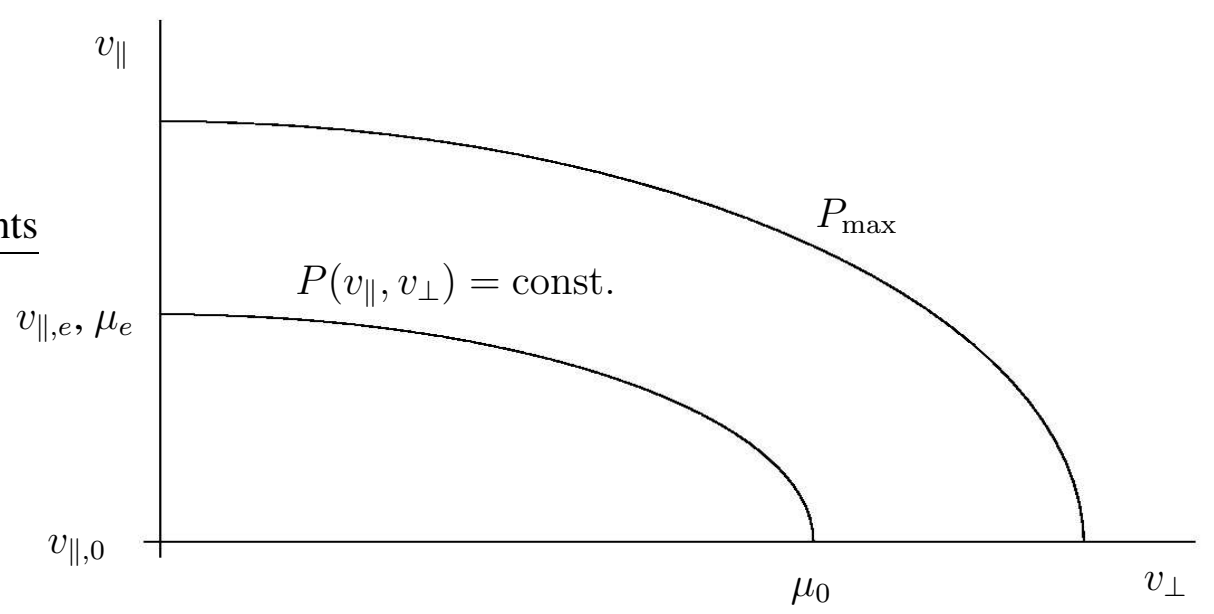

Abbildung 7.3: Skizze zur Bestimmung des Mittels einer Verteilung auf ihren Plateaus.

der Skizze 7.3 verwendet. Die Geschwindigkeit $v_{\perp}\left(P, v_{\|}\right)$ergibt sich als Lösung der Gleichung 7.6. Durch die Mittelung $\langle\cdot\rangle_{P}$ bleibt die Teilchendichte unberührt ${ }^{1}$. Die Norm

$$
\begin{aligned}
\|f\|^{2} & =2 \pi \int d v_{\|} d v_{\perp} v_{\perp} f^{2}\left(v_{\|}, v_{\perp}\right) \\
& =\pi \int d \mu d P P f^{2}(P, \mu) \\
& =\pi \int d P P \Delta \mu(P)\left\langle f^{2}\right\rangle_{P}(P)
\end{aligned}
$$

führt auf den Abstand

$$
\left\|f-\langle f\rangle_{P}\right\|^{2}=\pi \int d P P \Delta \mu(P)\left(\left\langle f^{2}\right\rangle_{P}(P)-\langle f\rangle_{P}^{2}(P)\right)
$$

zwischen der Geschwindigkeitsverteilung $f$ und ihrem Plateaumittel $\langle f\rangle_{P}$. Im Fall vollständig ausgebildeter Plateaus stimmen beide überein, und der Abstand verschwindet. $\mathrm{Als} \mathrm{Ma} \beta \epsilon$ für die Abweichung einer Geschwindigkeitsverteilung von den theoretischen Diffusionsplateaus verwende ich den relativen Abstand

$$
\epsilon=\left\|f-\langle f\rangle_{P}\right\| /\|f\| .
$$

Aus praktischen Gründen ist die Integration über die Plateauvariable $P$ in den Gleichungen 7.9 bis 7.12 durch einen maximalen Wert $P_{\max }$ beschränkt. Dadurch erstreckt sich die Geschwindigkeitsintegration auf den Bereich, der von einem äußersten Diffusionsplateau begrenzt wird (s. Skizze 7.3). Der Wert $P_{\max }$ wird auf der dem Protonenstrahl abgewandten Seite des Kerns der Verteilung so gewählt, daß die Positionen aller Gaußfunktionen, die in die verwendete Darstellung 4.1 der Geschwindigkeitsverteilung eingehen, noch im Integrationsgebiet liegen. Diese Positionen stimmen mit Kanalzentren des Instrumentes 1a überein (s. Abschnitt 3.1.1). Für die Diffusionsplateaus in der dem Protonenstrahl

\footnotetext{
${ }^{1}$ Somit ist die auf den theoretischen Diffusionsplateaus gemittelte Verteilung $\langle f\rangle_{P}$ der asymptotische Endzustand von f, wenn die Diffusion mit unveränderter Dispersion der beteiligten Wellen und unter Ausschluß anderer Einflüsse auf $f$ fortgesetzt würde.
} 


\begin{tabular}{cccccc}
\hline \hline$n_{p}\left(\mathrm{~cm}^{-3}\right)$ & $v_{\mathrm{sw}}(\mathrm{km} / \mathrm{s})$ & $v_{A}(\mathrm{~km} / \mathrm{s})$ & $\Omega_{p}\left(\mathrm{~s}^{-1}\right)$ & $\epsilon$ (abgewandt) & $\epsilon$ (zugewandt) \\
\hline 23.9 & 590 & 189 & 4.3 & $3.8 \times 10^{-2}$ & $1.1 \times 10^{-1}$ \\
5.7 & 614 & 109 & 1.1 & $3.6 \times 10^{-2}$ & $1.1 \times 10^{-1}$ \\
\hline \hline
\end{tabular}

Tabelle 7.1: Parameter zu den auf Helios 2 am 14. April 1976 um 10h/46m/8s und am 16. März 1976 um 4h/24m/52s in den Entfernungen von 0.29 AE bzw. 0.66 AE zur Sonne gemessenen Geschwindigkeitsverteilungen der Protonen (s.a. Abbildungen 7.2a-7.2d).

zugewandten Hälfte des Kerns kann das allerdings nicht erreicht werden, da die Resonanzgeschwindigkeiten der relevanten Zyklotronmoden beschränkt sind (s. Abbildungen 7.2a und 7.2b). In diesem Fall ist $P_{\max }$ durch die betraglich kleinste der in der numerischen Berechnung des Dispersionszweiges auftretenden Wellenzahlen $k_{\|, 0}$ bestimmt. Für diese Wellezahl kommt die Resonanzgeschwindigkeit dem Strahl am nächsten und $P_{\max }$ wird so gewählt, daß das der $v_{\|}$-Achsenabschnitt des entsprechenden Diffusionsplateaus mit der Resonanzgeschwindigkeit zu $k_{\|, 0}$ übereinstimmt. Die untere Integrationsgrenze für $P$ kann ohne Einschränkung der Allgemeinheit durch eine additive Konstante in der Gleichung 7.6 auf Null gesetzt werden. In der Tabelle 7.1 sind für die in den Abbildungen 7.1a und 7.1b sowie 7.2a-7.2d behandelten Beispiele die Abweichungen $\epsilon$ von den theoretischen Plateaus in den beiden Hälften des Kerns der Verteilungen angegeben. Die entsprechenden Werte von $\epsilon$ lauten für das im Abschnitt 2.3 diskutierte und in den Abbildungen 2.6a und 2.6b dargestellte Beispiel zum Vergleich $\epsilon=0.25$ und $\epsilon=0.3$ auf der dem Strahl ab- bzw. zugewandten Seite des Kerns der Verteilung. In diesem Fall besteht qualitativ wie quantitativ eine deutliche Abweichung der Verteilung von den theoretischen Plateaus. Die Beispiele vermitteln den erforderlichen Maßstab für die qualitative Bewertung der durch $\epsilon$ ausgedrückten Abweichung der 10804 ausgewerteten Verteilungsfunktionen von den theoretischen Diffusionsplateaus.

Die Abbildungen 7.4a-7.4d fassen die Ergebnisse der Auswertung für die ausgewählten schnellen Sonnenwindströmungen zusammen (s. Tabelle 3.1). Sie zeigen die auf Eins normierten Histogramme der Abweichungen $\epsilon$ der Verteilungen von den theoretischen Diffusionsplateaus und den Verlauf dieser Abweichungen mit der Entfernung zur Sonne. Die Teilabbildungen unterscheiden zwischen den Diffusionsplateaus, die in der dem Protonenstrahl ab- bzw. zugewandten Hälfte des Kerns der Verteilungen liegen. Diese gehören zu den auswärts laufenden Alfvén-Moden bzw. den einwärts laufenden Zyklotronmoden. Letztere sind in den Abbildungen 7.2a und 7.2b bei negativen Wellenzahlen zu sehen (s.a. Abbildung 2.6a). Der Mittelwert der Abweichungen aller ausgewerteten Geschwindigkeitsverteilungen von den theoretischen Diffusionsplateaus beträgt $5.8 \cdot 10^{-2}$ auf der dem Protonenstrahl abgewandten Seite des Kerns. Der entsprechende Mittelwert ist auf der dem Strahl zugewandten Seite mit 1.2 $10^{-1}$ deutlich größer. In den Abbildungen 7.4c und $7.4 \mathrm{~d}$ ist zu erkennen, daß die Abweichung der Verteilungen auf der dem Strahl zugewandten Seite in jeder Entfernung zur Sonne größer als auf der abgewandten Seite ist. Desweiteren variieren die Abweichungen der Verteilungen von den theoretischen Plateaus in der dem Protonenstrahl abgewandten Hälfte des Kerns nur unmerklich mit der Entfernung: $\bar{\epsilon}=0.05$ im Perihel und $\bar{\epsilon}=0.06$ im Aphel von Helios. Im Gegensatz dazu 
(a)

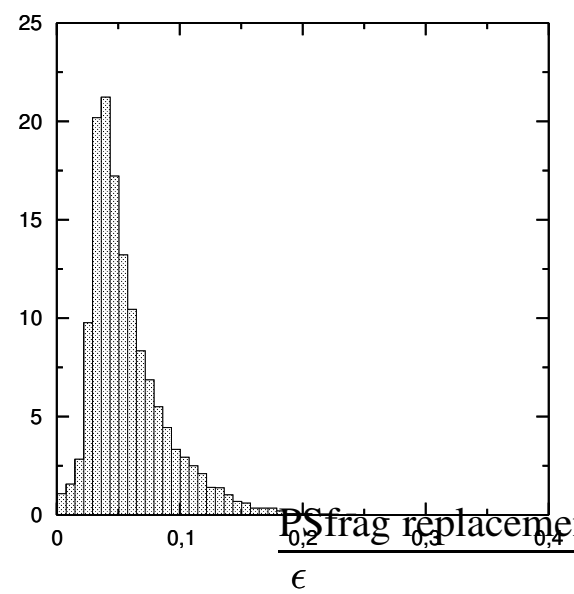

(c)

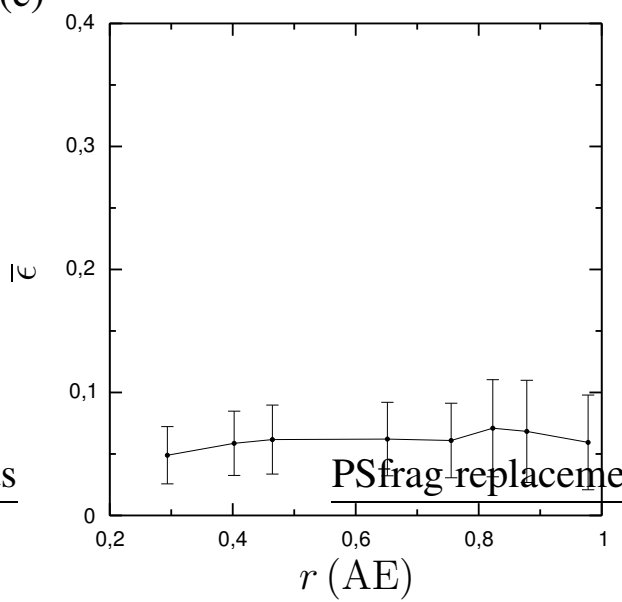

(b)

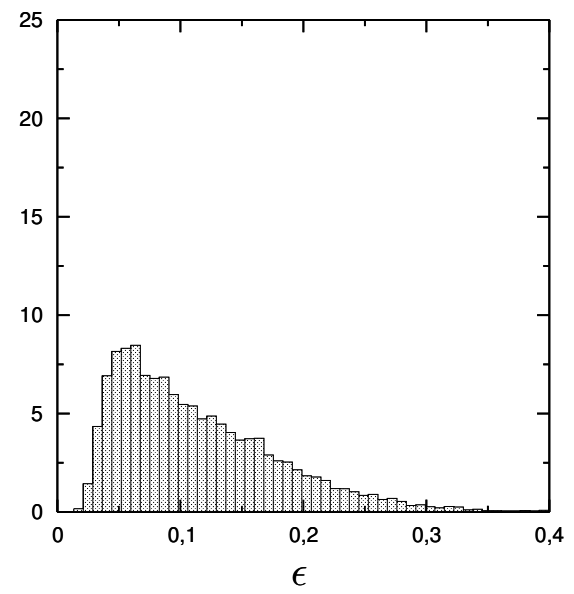

(d)

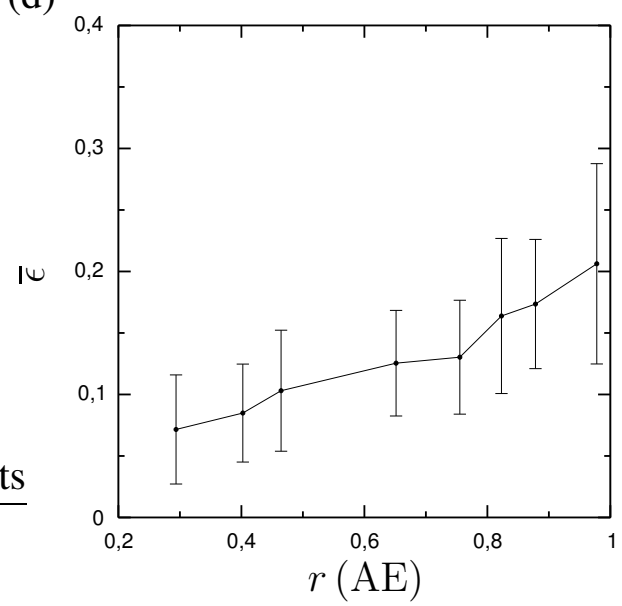

Abbildung 7.4: Auf Eins normierte Histogramme der Abweichungen $\epsilon$ aller innerhalb der ausgewählten schnellen Sonnenwindströmungen gemessenen Geschwindigkeitsverteilungen zu ihren Plateaumitteln in der dem Protonenstrahl (a) ab- und (b) zugewandten Hälfte des Kerns der Verteilungen. Die Mittelwerte und die Varianzen der Abweichungen $\epsilon$ in der dem Strahl ab- und abgewandten Hälfte innerhalb der Sonnenwindströmungen sind als Funktion der mittleren Entfernungen zur Sonne in den Abbildungen (c) bzw. (d) aufgetragen.

steigt auf der anderen Seite des Kerns die Abweichung mit der Entfernung deutlich an: $\bar{\epsilon}=0.07 \mathrm{im}$ Perihel und $\bar{\epsilon}=0.2 \mathrm{im}$ Aphel. Diese Ergebnisse zeigen, daß sich die Geschwindigkeitsverteilungen auf der dem Protonenstrahl abgewandten Seite des Kerns in allen Entfernungen zwischen dem Perihel und dem Aphel von Helios bei 0.29AE bzw. 1 AE nur gering von den theoretischen Diffusionsplateaus unterscheiden. Diese qualitative Beurteilung wird dadurch nahegelegt, daß die beobachteten Werte der Abweichung $\epsilon$ im Mittel mit den entsprechenden Werten der zu Beginn dieses Abschnitts besprochenen positiven Beispielen übereinstimmt. Die Varianz der Abweichung ist in dieser Hälfte des Kerns gering. Auf der dem Protonenstrahl zugewandten Seite des Kerns unterscheiden sich die Geschwindigkeitsverteilungen hingegen deutlich von den theoretischen Plateaus. Die Abweichung $\epsilon$ erreicht im Aphel von Helios Werte, die im Mittel mit der Abwei- 
chung $\epsilon$ des im Abschnitt 2.3 dieser Arbeit besprochenen Beispiels übereinstimmen. Für dieses Beispiel ist keine Ähnlichkeit zwischen den Höhenlinien der Verteilung und den theoretischen Plateaus zu erkennen.

Aus den Ergebnissen kann geschlußfolgert werden, daß die auswärts laufenden und mit den Protonen in der dem Strahl abgewandten Hälfte des Kerns der Verteilung resonanten Alfvén-Moden angeregt sein müssen. Sie sind für die beobachtete Temperaturanisotropie des Kerns der Geschwindigkeitsverteilungen der Protonen verantwortlich. Außerdem werden die Voraussetzungen der quasi-linearen Diffusionen im schnellen Sonnenwind weitgehend erfüllt sein. Der schnelle Sonnenwind ist schwach turbulent und es gilt eine Skalentrennung zwischen den Fluktuationen und dem Hintergrund der mittleren Felder. Die starken Abweichungen der Verteilungen von den theoretischen Plateaus auf der dem Protonenstrahl zugewandten Seite des Kerns müssen dadurch erklärt werden, daß die resonanten einwärts laufenden Zyklotronmoden mit einer zu niedrigen spektralen Energiedichte angeregt sind, um die Ausbildung von Plateaus zu erzwingen. In diesem Fall gewinnen die Advektion, etc. in der Bewegungsgleichung der Geschwindigkeitsverteilung an Bedeutung. Die nicht-linearen Korrekturen zur quasi-linearen Näherung dürfen wegen der im Kapitel 6 festgestellten linearen Instabilität eines breiten Bandes der auswärts laufenden Alfvén-Moden prinzipiell nicht vernachlässigt werden. Ihr Einfluß ist allerdings so gering, daß sie keine in den Beobachtungen benennbaren Abweichungen von den quasi-linearen Diffusionsplateaus verursachen. Dennoch unterschreiten auf beiden Seite des Kerns die Abweichungen der Geschwindigkeitsverteilungen von den theoretischen Plateaus nur in wenigen Fällen einen Wert von $\epsilon \approx 0.03$. Es ist unmöglich zu erkennen, ob sich dahinter ein systematische Fehler verbirgt, der durch die in dieser Arbeit verwendete Darstellung der Geschwindigkeitsverteilungen eingeführt wird. Durch die Wahl der im Kapitel 4 beschriebenen nicht-parametrischen Entwicklung der Geschwindigkeitsverteilungen nach Kernfunktionen habe ich versucht, den systematischen Fehler so gering wie möglich zu halten.

\subsection{Diffusionsplateaus im Strahl der Verteilung der Pro- tonen}

Tu et al. (2002) untersuchten die Möglichkeit, die Form des beobachteten Protonenstrahls ebenso wie die Form des Kerns der Verteilung durch Diffusionsplateaus zu erklären. Dafür suchten sie für ein kaltes und aus Elektronen, Protonen und $\alpha$-Teilchen bestehendes Plasma nach geeignete Dispersionszweigen der Zyklotronmoden. Einen Protonenstrahl bezogen sie nicht in die Dispersionsbeziehung ein, die für die Zyklotronmoden

$$
\omega^{2}+c^{2} k_{\|}^{2}+\sum_{s} \omega_{p s}^{2} \frac{\omega-v_{\|, s} k_{\|}}{\omega-v_{\|, s} k_{\|} \pm \Omega_{s}}=0
$$

lautet. Dabei sind $u_{s}$ und $\Omega_{s}$ die mittlere Geschwindigkeit bzw. die Zyklotronfrequenz der Teilchenart $s$. Diese Dispersionsbeziehung folgt aus derjenigen des warmen Plasmas durch den Grenzübergang $\beta_{s \|} \rightarrow 0,\left|\zeta_{s \pm}\right| \rightarrow \infty$ und die Näherung $Z(\zeta) \approx-1 / \zeta$ für $|\zeta| \gg 1$ und $\operatorname{Im} \zeta \geq 0$ (s. Abschnitt 2.2.2). Die Abbildung 7.5a zeigt die von Tu 
(a)

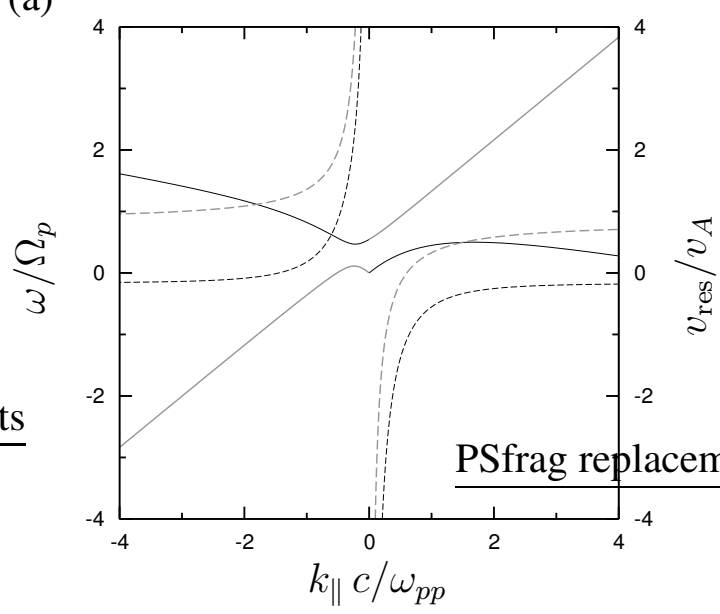

(b)

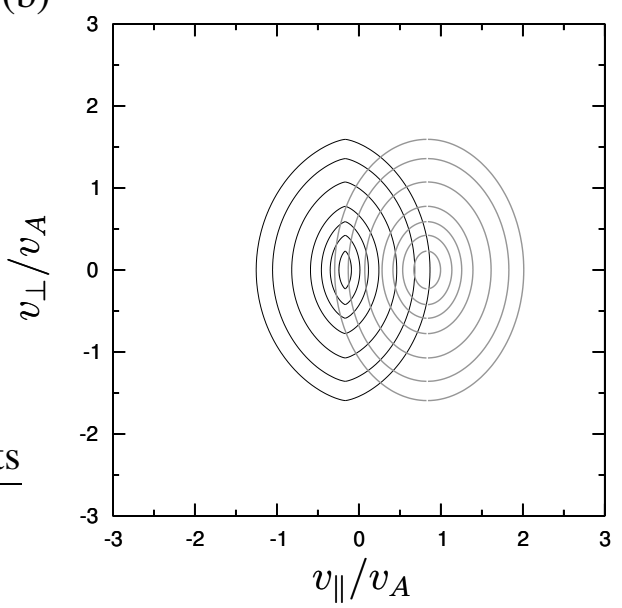

Abbildung 7.5: (a) Dispersionszweige des von Tu et al. (2002) betrachteten kalten Plasmas. (b) Die zu den Dispersionszweigen gehörenden Diffusionsplateaus.

et al. (2002) ausgewählten Dispersionszweige sowie die zu ihnen gehörenden Resonanzgeschwindigkeiten. Den Zweigen liegen die Parameter $n_{\alpha} / n_{p}=0.05$ und $v_{\|, \alpha}-v_{\|, p}=v_{A}$ zugrunde, durch die sie bis auf die Normierungen $\omega / \Omega_{p}$ und $c k_{\|} / \omega_{p p}$ vollständig bestimmt sind. Die Abbildung 7.5b stellt die den Zweigen entsprechenden theoretischen Diffusionsplateaus dar. Sie sind zum einen bei der mittleren Geschwindigkeit der Protonen $v_{\|, p}=0.17 v_{A}$ und zum anderen bei der mittleren Geschwindigkeit der $\alpha$-Teilchen $v_{\|, \alpha}=0.83 v_{A}$ zentriert. Letztere sollen nach der Meinung von Tu et al. (2002) den Protonenstrahl bestimmen. Allerdings ist es problematisch, daß zu jeder von $v_{\|, p}$ und $v_{\|, \alpha}$ verschiedenen Geschwindigkeit $v_{\|}$jeweils zwei Moden resonant sein können, weshalb sich die in der Abbildung 7.5 gezeigten Diffusionsplateaus überschneiden. Die Geschwindigkeitsverteilung der Protonen kann unter diesen Umständen nur stationär sein, wenn sie insgesamt konstant ist. Kern und Strahl der Verteilung können nicht bestehen, sondern sie zerlaufen im Geschwindigkeitsraum. Tu et al. (2002) vermieden dieses Problem, indem sie die spektralen Energiedichten der Moden der oberen Dispersionszweige so beschnitten, daß von zwei mit der gleichen Geschwindigkeit resonanten Moden nur eine angeregt ist. Sie schränkten die spektralen Energiedichten dazu willkürlich auf die Bereiche ein, in denen die Resonanzgeschwindigkeiten der Moden dieser Zweige bei negativen Wellenzahlen kleiner bzw. bei positiven Wellenzahlen größer als eine Grenzgeschwindigkeit in der Mitte zwischen zwischen $v_{\|}$und $v_{\alpha}$ sind. In dieser Arbeit habe ich anhand der bekannten Datenmenge (s. Abschnitt 3.2) für den schnellen Sonnenwindes untersucht, ob sich Diffusionsplateaus im Protonenstrahl der gemessenen Geschwindigkeitsverteilungen beobachten lassen. Ebenso wie Tu et al. (2002) betrachte ich nur sich parallel zum mittleren magnetischen Feld ausbreitende Zyklotronmoden. Deren Dispersion berechne ich aus den gemessenen Verteilungen (s. Erläuterungen im Abschnitt 7.1).

Die Abbildungen 7.6a und 7.6b zeigen für die beispielhaften Geschwindigkeitsverteilungen des vorherigen Abschnittes die im Abschnitt 2.2.2 diskutierten Dispersionszweige im Bereich der Zyklotronfrequenzen der Ionen (vgl. Abbildungen 7.2a, 7.2b). Zusätzlich zu den in der Abbildung 2.1b dargestellten Zweigen erscheinen zwei weitere, welche für kleine positive bzw. negative Wellenzahlen in eine Frequenz münden, die nicht wesent- 
(a)

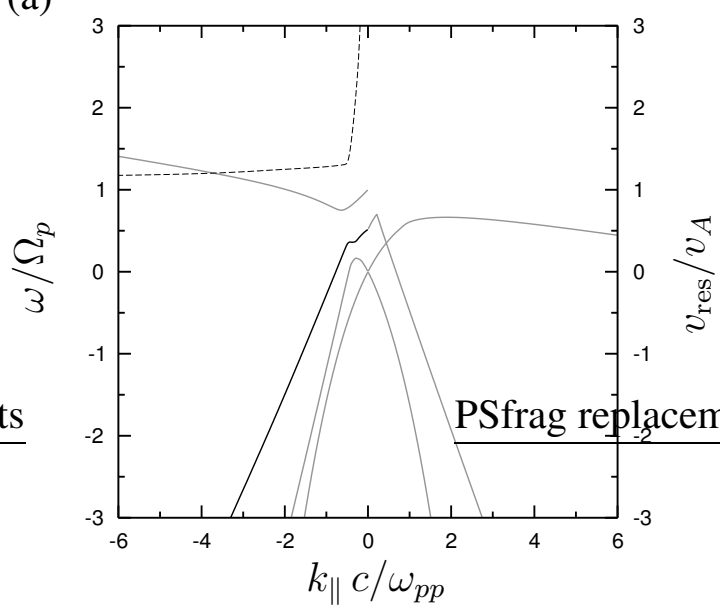

(c)

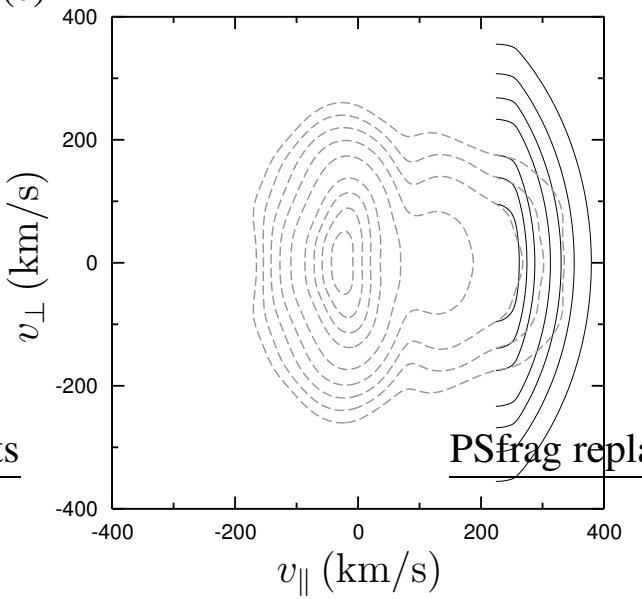

(b)

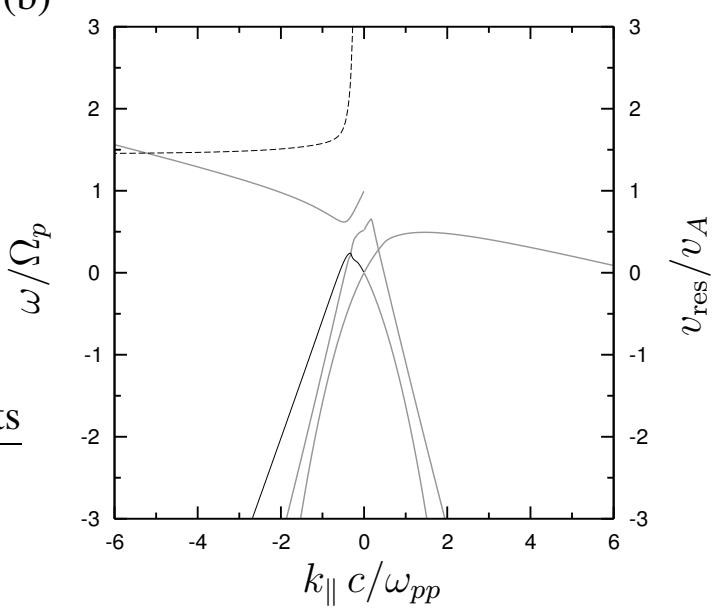

(d)

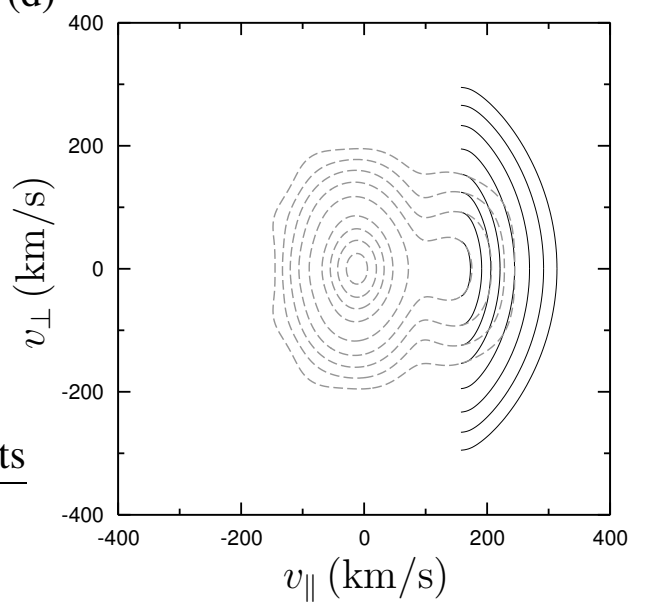

Abbildung 7.6: Die Abbildungen (a) und (b) zeigen Dispersionszweige zu den in den Abbildungen 7.1a bzw. 7.1b dargestellten und auf Helios 2 gemessenen Geschwindigkeitsverteilungen der Protonen. Die Dispersionszweige, deren Moden mit den Protonen im Strahl der Verteilungen in Resonanz treten, sind hervorgehoben (schwarz). Die betreffenden Resonanzgeschwindigkeiten, $v_{\text {res }}=\left(\omega-\Omega_{p}\right) / k_{\|}$, sind als gestrichelte Kurven zu sehen. Die Abbildungen (c) und (d) zeigen theoretische Diffusionsplateaus, welche zu den in (a) bzw. (b) hervorgehobenen Moden gehören. Die in den Abbildungen 7.1c bzw. 7.1d gezeichneten Höhenlinien der Verteilungen sind den Plateaus überlagert. Die Höhenlinien zum Anteil $10^{-3}$ des Maximums der Verteilungen sind hinzugefügt worden.

lich größer als die Zyklotronfrequenz der $\alpha$-Teilchen ist. Diese Dispersionszweige, die ihre Existenz den $\alpha$-Teilchen verdanken, entsprechen den in der Abbildung 7.5a für das kalte Plasma dargestellten oberen Dispersionzweigen. Im warmen Plasma sind die Moden auf beiden Zweigen bei betraglich kleinen Wellenzahlen ungedämpft. In den Abbildungen 7.6a und 7.6b sind diejenigen Dispersionszweige, deren Moden mit den Protonen im Strahl in Resonanz treten, hervorgehoben. Die Resonanzgeschwindigkeiten dieser Moden werden durch die gestrichelten Kurven dargestellt. Die Abbildungen 7.6a und 7.6b zeigen theoretische Diffusionsplateaus, welche aus den hervorgehobenen Moden abgeleitet sind. Die bereits in den Abbildungen 7.2c und 7.6d gezeichneten Höhenlinien der um das mitt- 
lere magnetische Feld symmetrisierten Verteilungen sind ebenfalls zu sehen. Es ist keine deutliche Übereinstimmung zwischen den Höhenlinien und den theoretischen Plateaus feststellbar. Unter den Dispersionszweigen, die nicht in den Abbildung 7.6a und 7.6b dargestellt sind, gibt es eine endliche Anzahl weiterer Zweige, deren Moden mit den Protonen im Strahl der Verteilungen in Resonanz treten. Die aus ihnen abgeleiteten theoretischen Diffusionsplateaus zeigen ebenfalls keine Übereinstimmung mit den Höhenlinien der jeweiligen Verteilung. Aus Gründen der Übersichtlichkeit, wurden diese Dispersionszweige und die entsprechen theoretischen Plateaus nicht in die Abbildungen 7.6a-7.6d aufgenommen. Die betreffenden Moden sind für alle Wellenzahlen stark gedämpft und vermutlich nicht angeregt.

Die Vorstellung von der Formung des Protonenstrahls durch die quasi-lineare Diffusion unter sich parallel zum mittleren magnetischen Feld ausbreitenden Zyklotronmoden läßt sich nicht durch Beobachtungen bestätigen. Für die beispielhaften Geschwindigkeitsverteilungen existieren keine Dispersionszweige, welche zu theoretischen Diffusionsplateaus führen, die mit den Höhenlinien der Verteilung im Strahl überzeugend übereinstimmen. Die Untersuchung einer großen Anzahl von Verteilungen wurde nicht versucht. Es kann kein eindeutiger Dispersionszweig benannt werden, der in allen Einzelfällen die Eigenschaft besitzt, mit dem Protonen im Strahl resonant zu sein (s.a. 7.6a und 7.6b). Die Betrachtung weiterer Einzelfälle ergab keine von diesen Feststellungen abweichende Ergebnisse. Es muß angenommen werden, daß die Wechselwirkung der Protonen mit den Moden der im Kapitel 6 nachgewiesenen Strahl-Instabilitäten für die Form des Protonenstrahls wichtiger ist. Allerdings kann die Diffusion der Protonen unter dieser Wechselwirkung offensichtlich nicht die Ursache für die Ausbildung des Strahls sein, wie es Tu et al. (2002) von den Zyklotronmoden mit paralleler Ausbreitungsrichtung angenommen haben. 


\section{Schlußfolgerungen und Ausblick}

Wesentliche Eigenschaften des schnellen Sonnenwindes lassen sich nicht ohne eine Wechselwirkung der Teilchen des Windes mit Plasmawellen erklären. Diese Wellen müssen im wesentlichen von der Sonne stammen und sich von ihr entlang des interplanetaren Magnetfeldes entfernen. Alfvén-Wellen mit großen Wellenlängen und Perioden, welche diese Eigenschaften besitzen, wurden im schnellen Sonnenwind nachgewiesen. Sie beschleunigen über ihren Wellendruck die Teilchen des Windes, ohne mit ihnen resonant zu sein, und erzeugen auf diese Weise die großen Strömungsgeschwindigkeiten des schnellen Sonnenwindes. In der Einleitung dieser Arbeit wurden die im schnellen Sonnenwind beobachtete Temperaturanisotropie der Protonen und die bevorzugte Beschleunigung sowie Heizung der Minoritätsionen angesprochen. Solche Abweichungen vom lokalen thermodynamischen Gleichgewicht sind dort durch die extrem kleinen Kollisionsfrequenzen möglich. Um ihr Entstehen zu erklären, müssen von der Sonne stammende Plasmawellen mit kleinen Wellenlängen und Perioden angenommen werden, die mit den Ionen resonant wechselwirken. Ihre Frequenzen sind von der Größenordnung der Zyklotronfrequenzen der Ionen. Neben der Sonne sind lineare Instabilitäten eine mögliche Quelle von Plasmawellen im interplanetaren Raum. Der im schnellen Sonnenwind stets vorhandene Protonenstrahl verursacht unter Umständen lineare Instabilitäten. Die auf diese Weise entstehenden Wellen haben durch die Relaxation ihrer Instabilität ggf. einen entscheidenden Einfluß auf die Geschwindigkeitsverteilungen der Teilchen des Windes. Die Strahl-Instabilitäten begrenzen somit die Geschwindigkeit des Protonenstrahls und können die Temperaturanisotropien des Strahls und des Kerns der Verteilung der Protonen mitbestimmen. Damit die Entwicklung des Sonnenwindes auf den großen Skalen zutreffend vorhergesagt werden kann, muß also sein turbulenter Zustand auf den kleinen Skalen verstanden werden. In dieser Hinsicht sind Beobachtungen erforderlich, die Aufschluß über seine wesentlichen Eigenschaften liefern und Anhaltspunkte für seine Modellierung liefern können. Es ist entscheidend zu wissen, welche der Plasmawellen im Bereich der Zyklotronfrequenzen der Ionen, die mit den Ionen resonant wechselwirken können, angeregt sind. In diesem Frequenzbereich liegen keine direkten Messungen von Plasmawellen vor. Die im Sonnenwind vorhandenen linearen Instabilitäten müssen identifiziert werden. Desweiteren sind auch die sich auf den keinen Skalen im Sonnenwind ausbreitenden Plasmawellen prinzipiell nicht frei von nicht-linearer Wechselwirkung. Die quasi-lineare Näherung, welche in Modellen des Sonnenwindes ausschließlich verwendet wird, braucht nicht auf alle Wellenmoden zuzutreffen. Auch die allgemeinen Vorstellungen einer schwachen Turbulenz und einer Skalentrennung zwischen den Fluktuationen und dem mittleren Zustand des Sonnenwindes benötigen eine Bestätigung. In dieser Arbeit hatte ich, soweit es die alleinige Messung der Geschwindigkeitsverteilungen der Protonen, Elektronen und $\alpha$-Teilchen 
zuläßt, diese Fragestellungen in Bezug auf den schnellen Sonnenwind untersucht.

Der erste Ansatzpunkt dieser Arbeit bestand in der Untersuchung des schnellen Sonnenwindes auf Mikroinstabilitäten. Diese Instabilitäten können nach ihren Ursachen, welche der Protonenstrahl bzw. die Temperaturanisotropie des Kerns der Verteilung der Protonen sind in zwei Kategorien unterteilt werden. Die Größenordnungen der beobachteten Anwachsraten der Instabilitäten erlauben Rückschlüsse auf die Bedeutung der nicht-linearen Wechselwirkung der instabilen Wellenmoden. Solche Wellenmoden, die sich quasi-linear verhalten, können höchstens marginal instabil sein. Die Untersuchung zeigte, daß die Strahl-Instabilitäten im schnellen Sonnenwind selten und nur für weniger als $10 \%$ der ausgewerteten Geschwindigkeitsverteilungen auftreten. Desweiteren sind die maximalen Anwachsraten der Strahl-Instabilitäten so gering, daß diese Instabilitäten im mittleren Zustand des schnellen Sonnenwindes höchstens marginal instabil sein können. Die Beobachtungen erlauben es nicht, zwischen der marginalen Instabilität und der absoluten Stabilität des Protonenstrahls zu unterscheiden. Im Unterschied dazu können die von der Temperaturanisotropie des Kerns der Verteilung der Protonen verursachten Instabilitäten aufgrund der Ergebnisse der angestellten Untersuchung nicht bloß marginal instabil sein. Insbesondere die Anwachsraten der in Bezug auf die Sonne auswärts laufenden Alfvén-Wellen im Bereich der Zyklotronfrequenzen der Ionen übersteigen die Grenze marginaler Instabilität um Größenordnungen. Zudem ergab sich diese Instabilität aus $90 \%$ aller ausgewerteten Geschwindigkeitsverteilungen. Während sich somit die vom Protonenstrahl im schnellen Sonnenwind verursachten Instabilitäten quasi-linear beschreiben lassen, kann die nichtlineare Wechselwirkung der auswärts laufenden Alfvén-Moden selbst auf den kleinen Skalen im Bereich der Zyklotronfrequenzen der Ionen nicht vernachlässigt werden. Das Überschreiten der Grenze ihrer absoluten Stabilität, was vor dem Perihel von Helios und unter Umständen noch innerhalb der Korona im Zusammenhang mit dem Entstehen der Temperaturanisotropie geschieht, ist mit der quasi-linearen Näherung genauso wie das beobachtete Verweilen im Bereich linearer Instabilität bis zur Erdentfernung unvereinbar. Eine Modenkopplung der instabilen Alfvén-Moden steht möglicherweise in einem Zusammenhang mit einer direkten Kaskade, die Energie von den groß- zu den kleinskaligen Fluktuationen befördern soll.

Der zweite Ansatzpunkt dieser Arbeit bestand in der Untersuchung der Geschwindigkeitsverteilungen der Protonen des schnellen Sonnenwindes auf Diffusionsplateaus, wie sie von der quasi-linearen Näherung der Welle-Teilchen-Wechselwirkung vorhergesagt werden. Der sogenannten quasi-linearen Diffusion liegen insbesondere die Vorstellung der schwachen Turbulenz und einer Skalentrennung zugrunde. Während sich im Kern der Verteilungen der Protonen Diffusionsplateaus nachweisen ließen, fand sich innerhalb des Protonenstrahls keine Übereinstimmung mit möglichen Diffusionsplateaus. Bei den mit den Protonen im Kern resonant wechselwirkenden Wellenmoden, welche die Diffusionsplateaus verursachen, handelt sich um die auswärts laufenden Alfvén-Moden Bereich der Zyklotronfrequenzen der Ionen bzw. um einen besonderen Zweig von einwärts laufenden Zyklotronmoden. Ein systematischer Vergleich zwischen den gemessenen Verteilungen und den theoretischen Diffusionsplateaus zeigte, daß die Verteilungen der Protonen zwischen dem Perihel und dem Aphel von Helios in der dem Protonenstrahl abgewandten Hälfte ihres Kerns unveränderlich gut mit den Diffusionsplateaus, welchen dort den auswärts laufenden Alfvén-Moden zuzuordnen sind, übereinstimmen. In der anderen 
Hälfte des Kerns der Verteilungen ist die Übereinstimmung mit den Diffusionsplateaus bereits im Perihel von Helios geringer. Zudem nehmen die Abweichungen mit wachsender Entfernung zur Sonne deutlich zu. Aus diesen Ergebnissen kann geschlußfolgert werden, daß die auswärts laufenden und mit den Protonen in der dem Strahl abgewandten Hälfte des Kerns resonanten Alfvén-Moden angeregt sein müssen. Sie sind für die beobachtete Temperaturanisotropie des Kerns der Geschwindigkeitsverteilungen der Protonen verantwortlich. Wegen der beobachteten linearen Instabilität eines breiten Bandes dieser Moden dürfen nicht-lineare Korrekturen zur quasi-linearen Näherung allerdings nicht vernachlässigt werden. Die Voraussetzungen der quasi-linearen Diffusion werden im schnellen Sonnenwind weitgehend erfüllt sein. Er ist insbesondere schwach turbulent und es gilt eine Skalentrennung zwischen den Fluktuationen und dem mittleren Zustand. Die starken Abweichungen der Verteilungen von den Diffusionsplateaus auf der dem Protonenstrahl zugewandten Seite des Kerns müssen dadurch erklärt werden, daß die entsprechenden einwärts laufenden Zyklotronmoden nicht hinreichend angeregt sind, um die Ausbildung von Plateaus zu erzwingen.

Die in dieser Arbeit angestellten Untersuchungen sollten auf den langsamen Sonnenwind ausgedehnt werden. Der langsame Wind unterscheidet sich allerdings in wichtigen Eigenschaften vom schnellen Sonnenwind. Die Differenz der mittleren Geschwindigkeit der $\alpha$-Teilchen und der Protonen ist im schnellen Sonnenwind nach den Messungen auf Helios nahezu parallel zum mittleren magnetischen Feld. Der Winkel zwischen beiden Richtungen streut gehäuft um Null. Eine von mir unternommene Auswertung von Messungen auf Helios im langsamen Sonnenwind ergab, daß dieser Winkel dort zwischen 0 und $\pi$ gleichverteilt ist. Eine zentrale Voraussetzung für diese Arbeit ist die gemeinsame Symmetrie der Geschwindigkeitsverteilungen aller Teilchenarten um das mittlere magnetische Feld, die somit im langsamen Wind verletzt zu sein scheint. Desweiteren besitzen die beobachteten Geschwindigkeitsverteilungen der Protonen im langsamen Sonnenwind eine deutlich variablere Gestalt. Es gibt Geschwindigkeitsverteilungen die keinen Protonenstrahl besitzen und nahezu isotrop sind. Andere Geschwindigkeitsverteilungen besitzen einen Protonenstrahl, dessen Teilchendichte mit derjenigen des Kerns der Verteilung vergleichbar ist. 



\section{A Radiale Profile der Plasmaparameter zu den untersuchten Strömungen schnellen Sonnenwindes}

In diesem Anhang sollen zur Übersicht der in dieser Arbeit verwendeten Meßdaten und der physikalischen Eigenschaften der untersuchten schnellen Sonnenwindströmungen die radialen Profile der wichtigen Plasmaparameter in einzelnen Abbildungen dargestellt werden. Die folgenden Abbildungen zeigen jeweils sowohl den Verlauf der Mittelwerte der Parameter in den Strömungen als Funktion der mittleren Entfernung der Messungen als auch die gemessenen Einzelwerte, um einen Eindruck von ihrer Streuung zu vermitteln. Obwohl die zusammengestellten Strömungen ihren Ursprung an verschiedenen Orten auf der Sonnenoberfläche haben und das gemessene Plasma zu verschiedenen Zeiten ausgeströmt ist, zeigen die Mittelwerte und Varianzen in den meisten Fällen einen systematischen Verlauf, wodurch die Eigenschaften des schnellen Sonnenwindes, einheitlich, stationär zu sein und sich mit den stets vergleichbaren Randbedingungen auf der Sonnenoberfläche im Gleichgewicht zu befinden, hervorgehoben werden (Axford und McKenzie 1992, 1997).

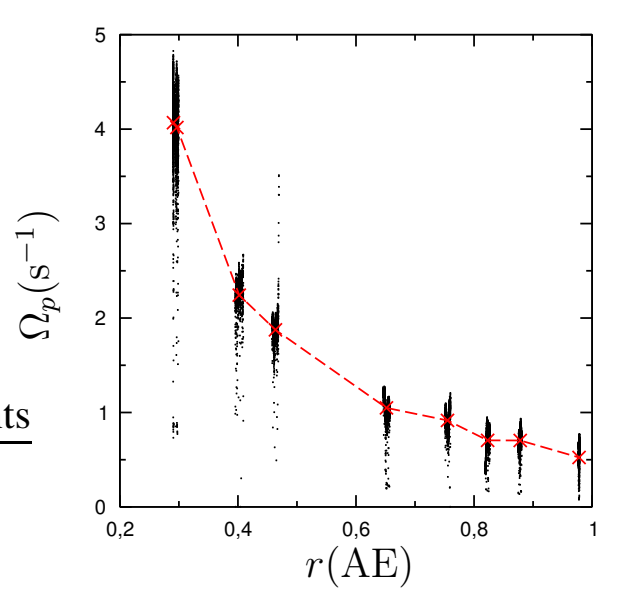

Abbildung A.1: Es ist die Zyklotronfrequenz der Protonen zu sehen. 
(a)

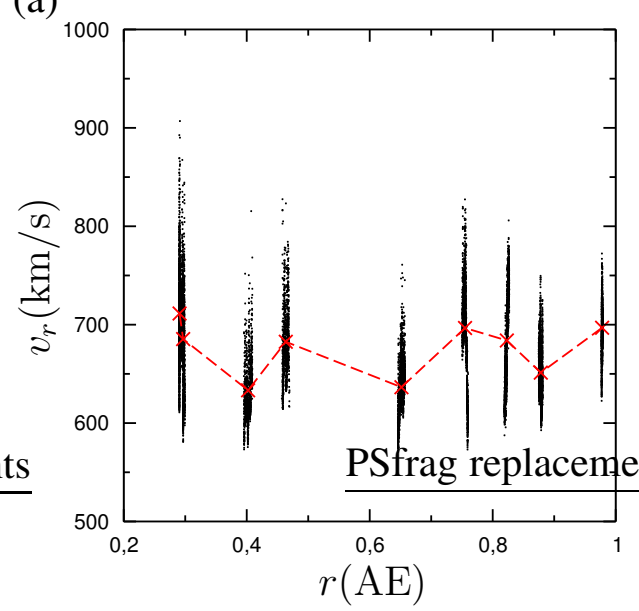

(c)

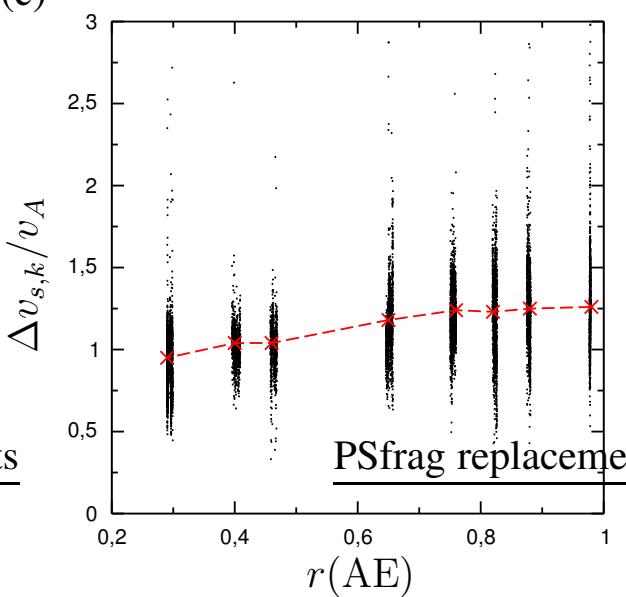

(e)

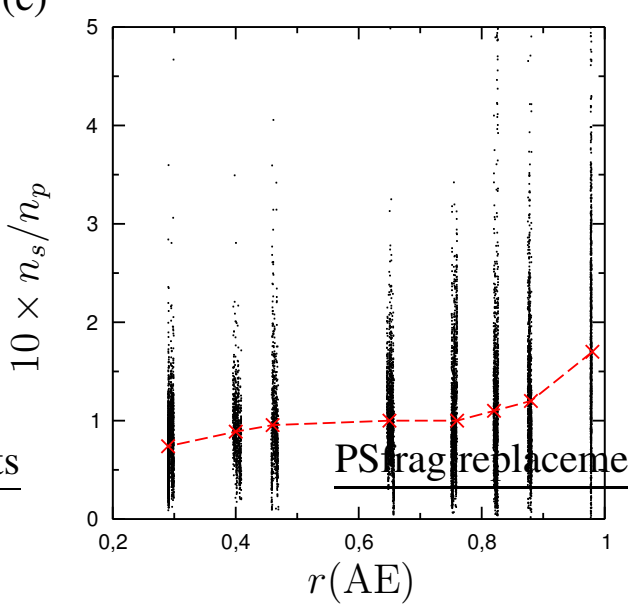

(b)

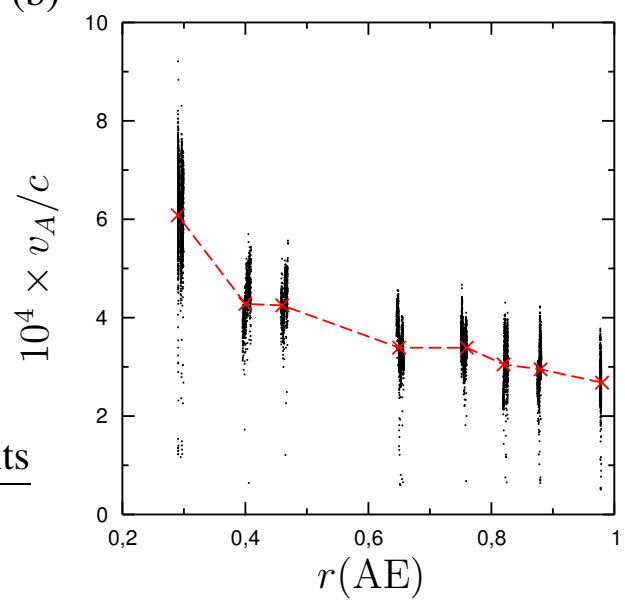

(d)

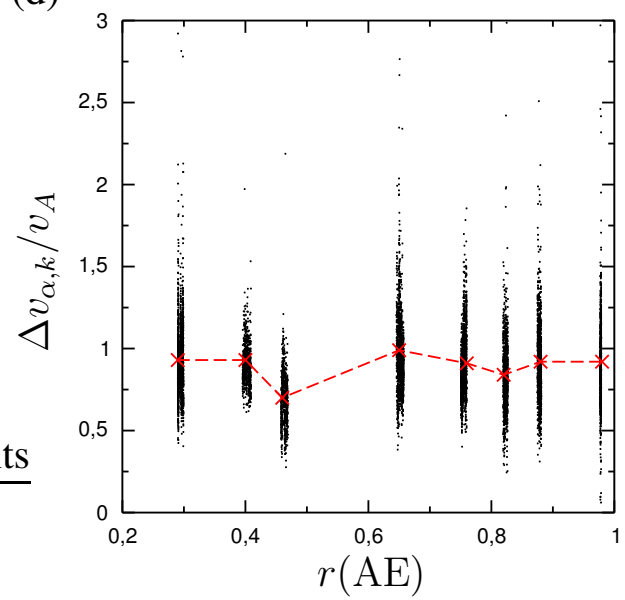

(f)

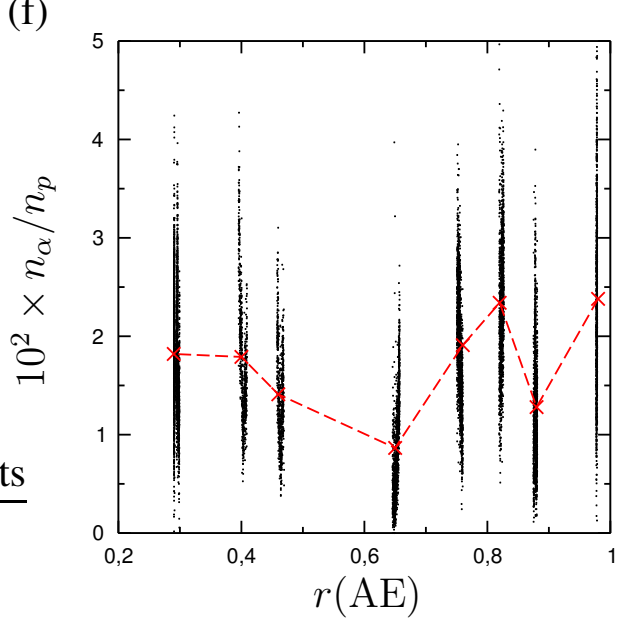

Abbildung A.2: Es sind (a) die radiale Komponente der Geschwindigkeit des Massenschwerpunktes der Protonen und $\alpha$-Teilchen, (b) die normierte Alfvén-Geschwindigkeit, (c),(d) die normierten Relativgeschwindigkeiten sowie (e),(f) die relativen Dichten des Protonenstrahls und der $\alpha$-Teilchen zu sehen. 
(a)

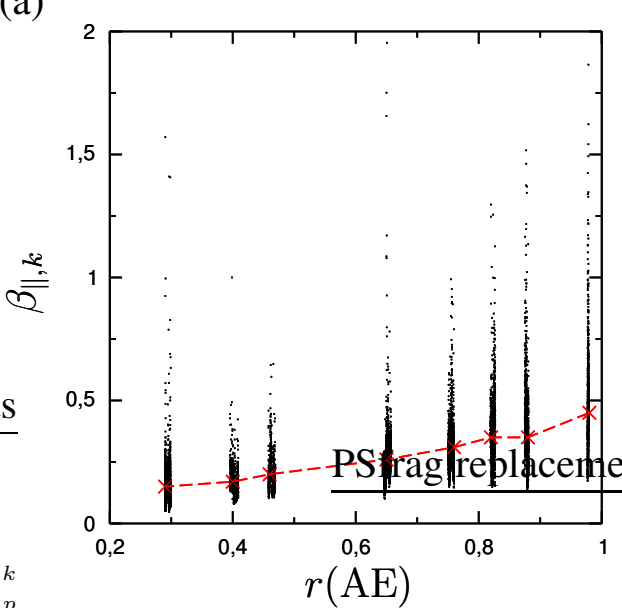

(c)

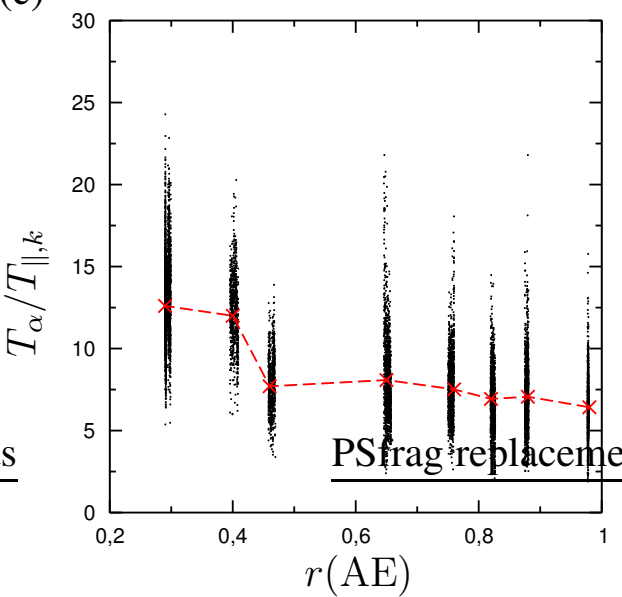

(e)

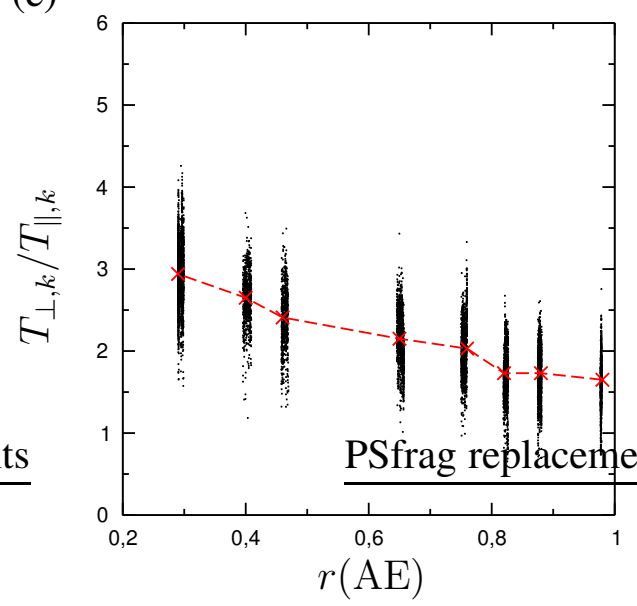

(b)

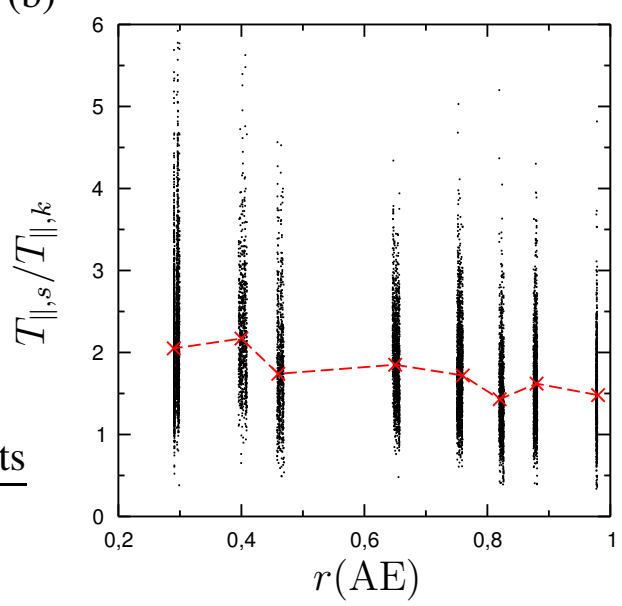

(d)

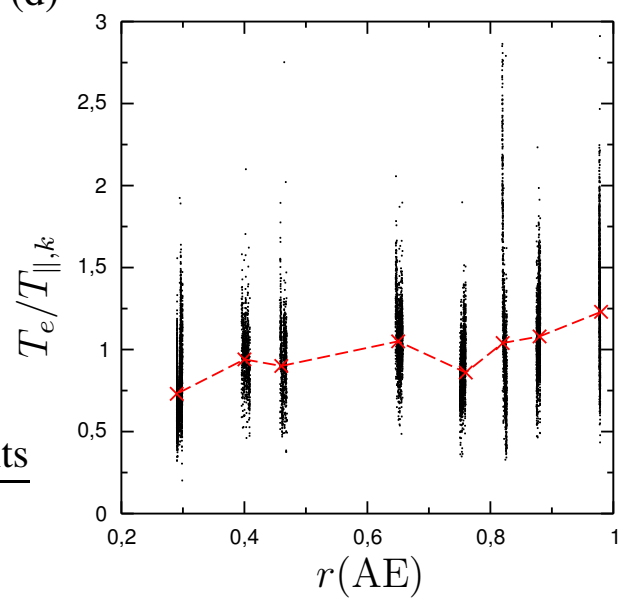

(f)

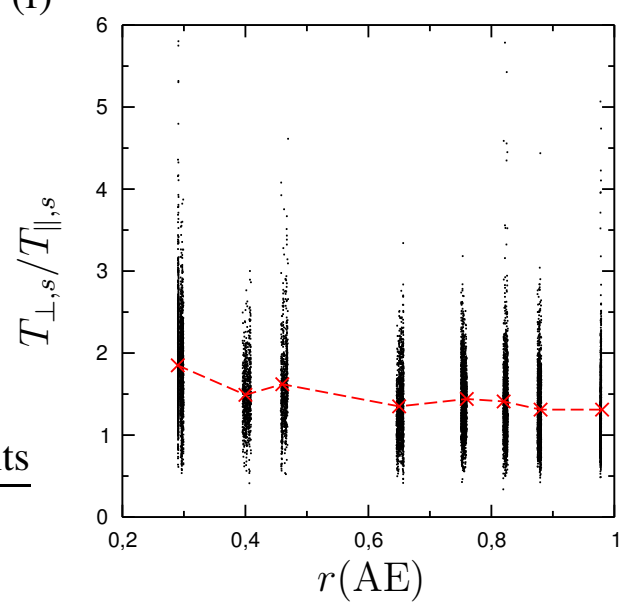

Abbildung A.3: Es sind (a) das Plasmabeta des Kerns der Protonenverteilung, (b),(c),(d) die relativen Temperaturen des Protonenstrahls, der $\alpha$-Teilchen und der Elektronen sowie (e),(f) die Temperaturanisotropien des Kerns und des Strahls der Protonenverteilung zu sehen. 


\begin{tabular}{|c|c|c|c|c|c|c|c|c|}
\hline Tag & $r(\mathrm{AE})$ & $\overline{v_{A} / c}$ & $\beta_{\|, k}$ & $\beta_{\|, p}$ & $T_{\perp, k} / T_{\|, k}$ & $n_{s} / n_{p}$ & $\Delta v_{k, s} / v_{A}$ & $T_{\|, s} / T_{\|, k}$ \\
\hline 23. Januar & 0.98 & $2.68 \times 10^{-4}$ & 0.45 & 0.93 & 1.65 & 0.17 & 1.26 & 1.48 \\
\hline 19. Februar & 0.88 & $2.95 \times 10^{-4}$ & 0.35 & 0.71 & 1.73 & 0.12 & 1.25 & 1.62 \\
\hline 27. Februar & 0.82 & $3.05 \times 10^{-4}$ & 0.35 & 0.66 & 1.73 & 0.11 & 1.23 & 1.43 \\
\hline 6. März & 0.76 & $3.39 \times 10^{-4}$ & 0.31 & 0.62 & 2.03 & 0.10 & 1.24 & 1.72 \\
\hline 16. März & 0.65 & $3.39 \times 10^{-4}$ & 0.26 & 0.54 & 2.15 & 0.10 & 1.18 & 1.85 \\
\hline 4. April & 0.40 & $4.28 \times 10^{-4}$ & 0.17 & 0.37 & 2.65 & $8.88 \times 10^{-2}$ & 1.04 & 2.17 \\
\hline 14./16. April & 0.29 & $6.08 \times 10^{-4}$ & 0.15 & 0.28 & 2.94 & $7.40 \times 10^{-2}$ & 0.95 & 2.05 \\
\hline 6. April 1977 & 0.46 & $4.25 \times 10^{-4}$ & 0.20 & 0.41 & 2.41 & $9.55 \times 10^{-2}$ & 1.04 & 1.74 \\
\hline Tag & $r(\mathrm{AE})$ & $T_{\perp, s} / T_{\|, s}$ & $n_{\alpha} / n_{p}$ & $\Delta v_{\alpha, k} / v_{A}$ & $\overline{T_{\alpha} / T_{\|, k}}$ & $T_{\alpha} / T_{\|, p}$ & $\overline{T_{e} / T_{\|, k}}$ & $T_{e} / T_{\|, p}$ \\
\hline 23. Januar & 0.98 & 1.31 & $2.38 \times 10^{-2}$ & 0.92 & 6.42 & 3.12 & 1.23 & 0.60 \\
\hline 19. Februar & 0.88 & 1.31 & $1.28 \times 10^{-2}$ & 0.92 & 7.06 & 3.47 & 1.08 & 0.53 \\
\hline 27. Februar & 0.82 & 1.41 & $2.34 \times 10^{-2}$ & 0.84 & 6.93 & 3.73 & 1.04 & 0.56 \\
\hline 6. März & 0.76 & 1.44 & $1.91 \times 10^{-2}$ & 0.91 & 7.51 & 3.81 & 0.86 & 0.44 \\
\hline 16. März & 0.65 & 1.35 & $8.65 \times 10^{-3}$ & 0.99 & 8.08 & 3.92 & 1.05 & 0.51 \\
\hline 4. April & 0.40 & 1.49 & $1.79 \times 10^{-2}$ & 0.93 & 12.0 & 5.69 & 0.94 & 0.45 \\
\hline 14./16. April & 0.29 & 1.85 & $1.82 \times 10^{-2}$ & 0.93 & 12.6 & 6.57 & 0.73 & 0.38 \\
\hline 6. April 1977 & 0.46 & 1.62 & $1.41 \times 10^{-2}$ & 0.70 & 7.70 & 3.85 & 0.90 & 0.45 \\
\hline
\end{tabular}

Tabelle A.1: Die Mittelwerte der normierten Parameter des Kerns und des Strahls der Verteilung der Protonen, der $\alpha$-Teilchen sowie der Elektronen in den zur Auswertung ausgewählten schnellen Sonnenwindströmungen des Jahres 1976 (vgl. Tabelle 3.1). 


\section{B Asymptotischer MISE für die nach Gaußschen Kernfunktionen entwickelten Geschwindigkeitsverteilungen}

In diesem Anhang soll der im Abschnitt 4.3 verwendete asymptotische MISE zu einer Entwicklung nach Gaußschen Kernfunktionen berechnet werden. Der Ausgangspunkt ist der Ansatz

$$
f(\vec{v})=\sum_{i=1}^{N} \frac{F_{i}}{h^{3}} K\left(\frac{\vec{v}-\vec{v}_{i}}{h}\right),
$$

worin $F_{i}, \vec{v}_{i}, i=1, \ldots, N$, beliebige Koeffizienten und Stützstellen sowie $h$ ein beliebiger Glättungsparameter sind. Der asymptotische MISE ist durch

$$
\begin{aligned}
A_{\mathrm{MISE}}=\frac{\delta_{N}}{h^{3}} \int d^{3} v \sigma^{2}(\vec{v}) \int d^{3} x K^{2}(\vec{x}) \\
+\frac{h^{4}}{4} \int d^{3} v\left(\sum_{i, j} \frac{\partial^{2} f}{\partial v_{i} \partial v_{j}}(\vec{v}) \int d^{3} x x_{i} x_{j} K^{2}(\vec{x})\right)^{2}
\end{aligned}
$$

vorgegeben (s. Abschnitt 4.2). Die enthaltenen Integrale der Kernfunktion

$$
K(\vec{x})=\frac{1}{(2 \pi)^{3 / 2}} e^{-\vec{x}^{2} / 2}
$$

lauten

$$
\int d^{3} x K^{2}(\vec{x})=(4 \pi)^{-3 / 2}
$$

und

$$
\int d^{3} x x_{i} x_{j} K(\vec{x})^{2}=\delta_{i j}
$$

Es sei

$$
K_{1}(x)=\frac{1}{\sqrt{2 \pi}} e^{-x^{2} / 2} .
$$


Dann gilt

$$
\begin{aligned}
\sum_{i} \frac{\partial^{2} f}{\partial v_{i}^{2}} & =\sum_{i}^{N} F_{i} \sum_{k=1}^{3} \frac{1}{h^{3}} K_{1}^{\prime \prime}\left(\frac{v_{k}-v_{i k}}{h}\right) \prod_{j \neq k} \frac{1}{h} K_{1}\left(\frac{v_{j}-v_{i j}}{h}\right) \\
& =\sum_{i}^{N} F_{i} \sum_{k=1}^{3} \frac{1}{h^{2}}\left(\left(\frac{v_{k}-v_{i k}}{h}\right)^{2}-1\right) \prod_{j=1}^{n} \frac{1}{h} K_{1}\left(\frac{v_{j}-v_{i j}}{h}\right) \\
& =\sum_{i}^{N} \frac{F_{i}}{h^{2}}\left(\left|\frac{\vec{v}-\vec{v}_{i}}{h}\right|^{2}-3\right) \frac{1}{h^{3}} K\left(\frac{\vec{v}-\vec{v}_{i}}{h}\right)
\end{aligned}
$$

wofür

$$
K_{1}^{\prime \prime}(x)=\left(x^{2}-1\right) \cdot K_{1}(x)
$$

verwendet wurde. Daraus folgen

$$
\begin{aligned}
\left(\sum_{i} \frac{\partial^{2} f}{\partial v_{i}^{2}}\right)^{2}= & \sum_{i, j}^{N} \frac{F_{i} F_{j}}{(2 \pi)^{3} h^{10}}\left(\left|\frac{\vec{v}-\vec{v}_{i}}{h}\right|^{2}-3\right) \\
& \times\left(\left|\frac{\vec{v}-\vec{v}_{j}}{h}\right|^{2}-3\right) e^{-\left(\left(\vec{v}-\vec{v}_{i}\right)^{2}+\left(\vec{v}-\vec{v}_{j}\right)^{2}\right) / 2 h^{2}}
\end{aligned}
$$

und

$$
\begin{aligned}
\int d^{3} v\left(\sum_{i} \frac{\partial^{2} f}{\partial v_{i}^{2}}\right)^{2} & =\sum_{i, j}^{N} \frac{F_{i} F_{j}}{(2 \pi)^{3} h^{10}} \int d^{3} v\left(\left|\frac{\vec{v}-\vec{v}_{i}}{h}\right|^{2}-3\right) \\
& \times\left(\left|\frac{\vec{v}-\vec{v}_{j}}{h}\right|^{2}-3\right) e^{-\left(\left(\vec{v}-\vec{v}_{i}\right)^{2}+\left(\vec{v}-\vec{v}_{j}\right)^{2}\right) / 2 h^{2}} \\
& =\sum_{i, j}^{N} \frac{F_{i} F_{j}}{(2 \pi)^{3} h^{10}} e^{-\vec{u}_{i j}^{2}} \int d^{3} v\left(\left|\frac{\vec{v}-\vec{v}_{i}}{h}\right|^{2}-3\right) \\
& =\sum_{i, j}^{N} \frac{F_{i} F_{j}}{(2 \pi)^{3} h^{7}} e^{-\vec{u}_{i j}^{2}} \int d^{3} v\left(\left|\vec{v}+\vec{u}_{i j}\right|^{2}-3\right) \\
= & \left.\left.\sum_{i, j}^{N} \frac{F_{i} F_{j}}{(2 \pi)^{3} h^{7}} e^{-\vec{u}_{i j}^{2}}\right|^{2}-3\right) e^{-\left(\vec{v}-\left(\vec{v}_{i}+\vec{v}_{j}\right) / 2\right)^{2} / h^{2}} \\
& \left.\left.-4\left(\mid \vec{v} \cdot \vec{u}_{i j}\right)^{2}+2 \vec{u}_{i j}^{2} \vec{v}^{2}-6\left(\vec{v}^{2}+\vec{u}_{i j}^{2}\right)+9\right)\left.\vec{u}_{i j}\right|^{2}-3\right) e^{-\vec{v}^{2}},
\end{aligned}
$$

wobei die Abkürzung $\vec{u}_{i j}=\left(\vec{v}_{i}-\vec{v}_{j}\right) / 2 h$ verwendet wird. Die beteiligten Integrale lauten

$$
\int d^{3} v|\vec{v}|^{4} e^{-\vec{v}^{2}}=\frac{15}{4} \pi^{3 / 2},
$$




$$
\int d^{3} v(\vec{v} \cdot \vec{u})^{2} e^{-\vec{v}^{2}}=\frac{\pi^{3 / 2}}{2} \vec{u}^{2}
$$

und

$$
\int d^{3} v \vec{v}^{2} e^{-\vec{v}^{2}}=\frac{3 \pi^{3 / 2}}{2} .
$$

Das Einsetzen in die Ausgangsgleichung führt auf das Ergebnis

$$
\begin{aligned}
& \int d^{3} v\left(\sum_{i} \frac{\partial^{2} f}{\partial v_{i}^{2}}\right)^{2}= \sum_{i, j}^{N} \frac{F_{i} F_{j}}{(2 \pi)^{3} h^{7}} e^{-\vec{u}_{i j}^{2}} \int d^{3} v\left(|\vec{v}|^{4}+\left|\vec{u}_{i j}\right|^{4}\right. \\
&\left.-4\left(\vec{v} \cdot \vec{u}_{i j}\right)^{2}+2 \vec{u}_{i j}^{2} \vec{v}^{2}-3\left(\vec{v}^{2}+\vec{u}_{i j}^{2}\right)+9\right) e^{-\vec{v}^{2}} \\
&= \sum_{i, j}^{N} \frac{F_{i} F_{j}}{(4 \pi)^{3 / 2} h^{7}}\left(\frac{15}{4}+\left|\vec{u}_{i j}\right|^{4}-2 \vec{u}_{i j}^{2}+3 \vec{u}_{i j}^{2}\right. \\
&\left.-3\left(\frac{3}{2}+\vec{u}_{i j}^{2}\right)+9\right) e^{-\vec{u}_{i j}^{2}} \\
&= \sum_{i, j}^{N} \frac{F_{i} F_{j}}{(4 \pi)^{3 / 2} h^{7}}\left(\left|\vec{u}_{i j}\right|^{4}-5\left|\vec{u}_{i j}\right|^{2}+\frac{15}{4}\right) e^{-\vec{u}_{i j}^{2}}
\end{aligned}
$$

welches sich zusammen mit dem Polynom

$$
P(x)=x^{4}-5 x^{2}+\frac{15}{4}
$$

in der kompakteren Form

$$
\int d^{3} v\left(\sum_{i} \frac{\partial^{2} f}{\partial v_{i}^{2}}\right)^{2}=\sum_{i, j}^{N} \frac{F_{i} F_{j}}{(4 \pi)^{3 / 2} h^{7}} P\left(\frac{\vec{v}_{i}-\vec{v}_{j}}{2 h}\right) e^{-\left(\vec{v}_{i}-\vec{v}_{j}\right)^{2} / 4 h^{2}}
$$

schreiben läßt. Der asymptotische MISE lautet damit

$$
\begin{aligned}
A_{\mathrm{MISE}}=\frac{\delta_{N}}{(4 \pi)^{3 / 2} h^{3}} & \int d^{3} v \sigma^{2}(\vec{v}) \\
& +\frac{1}{4} \sum_{i, j}^{N} \frac{F_{i} F_{j}}{(4 \pi)^{3 / 2} h^{3}} P\left(\frac{\vec{v}_{i}-\vec{v}_{j}}{2 h}\right) e^{-\left(\vec{v}_{i}-\vec{v}_{j}\right)^{2} / 4 h^{2}} .
\end{aligned}
$$





\section{Symmetrisierung und Reduktion der nach Gaußschen Kernfunktionen entwickelten Geschwindigkeitsverteilungen}

In diesem Anhang sollen die reduzierten Geschwindigkeitsverteilungen der nach Gaußschen Kernfunktionen entwickelten Verteilungen berechnet werden. Der Ausgangspunkt ist die Darstellung

$$
f(\vec{v})=\sum_{i=1}^{N} \frac{F_{i}}{h^{3}} K\left(\frac{\vec{v}-\vec{v}_{i}}{h}\right),
$$

worin $F_{i}, \vec{v}_{i}, i=1, \ldots, N$, beliebige Koeffizienten und Stützstellen sowie $h$ ein beliebiger Glättungsparameter sind. Die Kernfunktion lautet

$$
K(\vec{x})=\frac{1}{(2 \pi)^{3 / 2}} e^{-\vec{x}^{2} / 2} .
$$

Die reduzierte Geschwindigkeitsverteilung $n$-ter Ordnung einer um das magnetische Feld $\vec{B}$ symmetrischen Verteilung $F\left(v_{\|}, v_{\perp}\right)$ ist durch

$$
F_{n}\left(v_{\|}\right)=2 \pi \int_{0}^{\infty} d v_{\perp} v_{\perp}^{2 n+1} F\left(v_{\|}, v_{\perp}\right)
$$

definiert. Der Ansatz C.1 besitzt die Symmetrieeigenschaft nicht und muß erst symmetrisiert werden

$$
F\left(v_{\|}, v_{\perp}\right)=\int_{0}^{2 \pi} \frac{d \phi}{2 \pi} f\left(\vec{u}+\vec{v}\left(v_{\|}, v_{\perp}, \phi\right)\right)
$$

wobei

$$
\vec{v}\left(v_{\|}, v_{\perp}, \phi\right)=v_{\|} \vec{e}_{0}+v_{\perp} \cos \phi \vec{e}_{1}+v_{\perp} \sin \phi \vec{e}_{2}
$$

und $\vec{u}$ ein Bezugspunkt für die Symmetrisierung, z.B. die mittlere Geschwindigkeit der Verteilung oder die Geschwindigkeit des Massenschwerpunktes aller Teilchensorten, sind. Die Basis $\vec{e}_{0}, \vec{e}_{1}, \vec{e}_{2}$ ist orthonormal und es gilt $\vec{e}_{0}=\vec{B} / B$. 


\section{Symmetrisierung}

In einem ersten Schritt soll die Gyrophasenmittelung ausgeführt werden. Mit der Bezeichnung

$$
\vec{u}_{i}=\vec{v}_{i}-\vec{u}=u_{i \|} \vec{e}_{0}+u_{i \perp} \cos \psi_{i} \vec{e}_{1}+u_{i \perp} \sin \psi_{i} \vec{e}_{2}
$$

gilt

$$
\begin{aligned}
f\left(\vec{u}+\vec{v}\left(v_{\|}, v_{\perp}, \phi\right)\right)= & \left(2 \pi h^{2}\right)^{-3 / 2} \sum_{i=1}^{N} F_{i} e^{-\left(\vec{v}-\vec{v}_{i}+\vec{u}\right)^{2} / 2 h^{2}} \\
= & \left(2 \pi h^{2}\right)^{-3 / 2} \sum_{i=1}^{N} F_{i} e^{-\left(v_{\|}-u_{i \|}\right)^{2} / 2 h^{2}} \\
& \times e^{-\left(v_{\perp}^{2}+u_{i \perp}^{2}-2 v_{\perp} u_{i \perp} \cos \left(\phi-\psi_{i}\right)\right) / 2 h^{2}} .
\end{aligned}
$$

Das Integral

$$
\int_{0}^{2 \pi} \frac{d \phi}{2 \pi} e^{z \cos \phi}
$$

läßt sich durch die Identität

$$
e^{z \cos \phi}=\sum_{k=-\infty}^{\infty} J_{k}(i z) e^{i k(\phi-\pi / 2)}, \quad z \in \mathbb{C}
$$

der Besselfunktionen erster Art (Gleichungen 9.6.34 Abramowitz und Stegun 1965) berechnen und es ergibt sich

$$
\int_{0}^{2 \pi} \frac{d \phi}{2 \pi} e^{z \cos \phi}=J_{0}(i z)
$$

Daraus folgt sofort

$$
\begin{aligned}
F\left(v_{\|}, v_{\perp}\right) & =\int_{0}^{2 \pi} \frac{d \phi}{2 \pi} f\left(\vec{u}+\vec{v}\left(v_{\|}, v_{\perp}, \phi\right)\right) \\
& =\left(2 \pi h^{2}\right)^{-3 / 2} \sum_{i=1}^{N} F_{i} J_{0}\left(\frac{i v_{\perp} u_{i \perp}}{h^{2}}\right) e^{-\left(v_{\|}-u_{i \|}\right)^{2} / 2 h^{2}} e^{-\left(v_{\perp}^{2}+u_{i \perp}^{2}\right) / 2 h^{2}}
\end{aligned}
$$

\section{Reduktion}

Aus der Symmetrisierung C.11 lassen sich nun die reduzierten Verteilungen C.3 berechnen. Das entscheidende Integral ist

$$
R_{n}(w, z)=\int_{0}^{\infty} d v_{\perp} v_{\perp}^{2 n+1} e^{-w v_{\perp}^{2}} J_{0}\left(z v_{\perp}\right) .
$$


Es gilt

$$
R_{n}(w, z)=(-1)^{n} \frac{\partial^{n}}{\partial w^{n}} \int_{0}^{\infty} d v_{\perp} v_{\perp} e^{-w v_{\perp}^{2}} J_{0}\left(z v_{\perp}\right),
$$

weshalb die Integration nur für $n=0$ ausgeführt zu werden braucht. Hierzu wird die Potenzreihe der Besselfunktion

$$
J_{0}(z)=\sum_{k=0}^{\infty} \frac{(-1)^{k}}{k ! \Gamma(k+1)} z^{2 k}
$$

verwendet. Durch die Vertauschung von Integration und Summation folgt

$$
\begin{aligned}
R_{0}(w, z) & =\int_{0}^{\infty} d v_{\perp} v_{\perp} e^{-w v_{\perp}^{2}} \sum_{k=0}^{\infty} \frac{(-1)^{k}}{k !^{2}}\left(\frac{z v_{\perp}}{2}\right)^{2 k} \\
& =\sum_{k=0}^{\infty} \frac{(-1)^{k}(z / 2)^{2 k}}{k !^{2}} \int_{0}^{\infty} d v_{\perp} v_{\perp}^{2 k+1} e^{-w v_{\perp}^{2}} \\
& =\sum_{k=0}^{\infty} \frac{(-1)^{k}(z / 2)^{2 k}}{2 k ! w^{k+1}} \\
& =\frac{1}{2 w} e^{-z^{2} / 4 w} .
\end{aligned}
$$

Mit diesem Ergebnis schreiben sich die reduzierten Verteilungen

$$
\begin{gathered}
F_{n}\left(v_{\|}\right)=\left(2 \pi h^{2}\right)^{-1 / 2} \sum_{i=1}^{N} F_{i}(-1)^{n} \frac{e^{-u_{i \perp}^{2} / 2 h^{2}}}{h^{2}} \frac{\partial^{n} R_{0}}{\partial w^{n}}\left(\frac{1}{2 h^{2}}, \frac{i u_{i \perp}}{h^{2}}\right) \\
\times e^{-\left(v_{\|}-u_{i \|}\right)^{2} / 2 h^{2}} .
\end{gathered}
$$

Insbesondere gelten in nullter Ordnung

$$
F_{0}\left(v_{\|}\right)=\left(2 \pi h^{2}\right)^{-1 / 2} \sum_{i=1}^{N} F_{i} e^{-\left(v_{\|}-u_{i \|}\right)^{2} / 2 h^{2}}
$$

und in erster Ordnung

$$
F_{1}\left(v_{\|}\right)=\left(2 \pi h^{2}\right)^{-1 / 2} \sum_{i=1}^{N} 2 h^{2} F_{i}\left(1+\frac{u_{i \perp}^{2}}{2 h^{2}}\right) e^{-\left(v_{\|}-u_{i \|}\right)^{2} / 2 h^{2}},
$$

was aus

$$
\frac{\partial R_{0}}{\partial w}(w, z)=-\frac{1}{2 w^{2}}\left(1-\frac{z^{2}}{4 w}\right) e^{-z^{2} / 4 w}
$$

folgt. 



\section{Dispersionsbeziehung für die nach Gaußschen Kernfunktionen entwickelten reduzierten Geschwindigkeitsverteilungen}

In diesem Anhang sollen die Dispersionsfunktionen von sich parallel zum magnetischen Feld ausbreitenden elektrostatischen

$$
D_{\|}\left(\omega, k_{\|}\right)=\omega^{2}+\sum_{s} \frac{\omega_{p s}^{2}}{n_{s}} \int d v_{\|} \frac{\omega v_{\|}}{\omega-k_{\|} v_{\|}} \frac{\partial F_{s, 0}}{\partial v_{\|}}
$$

und Zyklotron-Moden

$$
\begin{aligned}
D_{ \pm}\left(\omega, k_{\|}\right) & =\omega^{2}-c^{2} k_{\|}^{2}+\sum_{s} \frac{\omega_{p s}^{2}}{n_{s}} \int d v_{\|} \frac{k_{\|} / 2}{\omega-k_{\|} v_{\|} \pm \Omega_{s}} \frac{\partial F_{s, 1}}{\partial v_{\|}} \\
& -\sum_{s} \frac{\omega_{p s}^{2}}{n_{s}} \int d v_{\|} \frac{\omega-k_{\|} v_{\|}}{\omega-k_{\|} v_{\|} \pm \Omega_{s}} F_{s, 0}
\end{aligned}
$$

für reduzierte Verteilungen $F_{s, 0}$ und $F_{s, 1}$ der Teilchenspezies $s$ der Form

$$
F_{s, n}\left(v_{\|}\right)=\sum_{i} F_{s, n}^{i}\left(\pi / a_{s, n}^{i}\right)^{-1 / 2} e^{-a_{s, n}^{i}\left(v_{\|}-v_{s, n}^{i}\right)^{2}}
$$

bestimmt werden. Die Koeffizienten $F_{s, n}^{i}$, die Stützstellen $v_{s, n}^{i}$ und die Breiten $a_{s, n}^{i}$ sollen in den folgenden Herleitungen beliebig sein. Im speziellen gelten aber für die im Anhang $\mathrm{C}$ berechneten reduzierten Verteilungen der nach gaussischen Kernfunktionen entwickelten Geschwindigkeitsverteilungen mit den dortigen Notationen

$$
\begin{aligned}
& F_{0}^{i}=F_{i} \\
& F_{1}^{i}=2 h^{2} F_{i}\left(1+\frac{u_{i \perp}^{2}}{2 h^{2}}\right)
\end{aligned}
$$

$v_{0}^{i}=v_{1}^{i}=u_{i \|}$ und $a_{0}^{i}=a_{1}^{i}=1 / 2 h^{2}$ für alle $i=1, \ldots, N$, wobei der Einfachheit halber der Teilchenindex $s$ ausgelassen wurde. Für die ebenfalls in dieser Arbeit oft verwendeten Bi-Maxwell-Verteilungen

$$
F\left(v_{\|}, v_{\perp}\right)=\left(\frac{m}{2 \pi k_{B}}\right)^{3 / 2} \frac{n}{T_{\perp} \sqrt{T_{\|}}} e^{-\left(m / 2 k_{B} T_{\|}\right)\left(v_{\|}-u_{\|}\right)^{2}} e^{-\left(m / 2 k_{B} T_{\perp}\right) v_{\perp}^{2}}
$$


von Teilchen der Masse $m$, der Teilchendichte $n$ und der Temperaturen $T_{\|}, T_{\perp}$ parallel bzw. senkrecht zum magnetischen Feld gelten $F_{0}^{1}=n, F_{1}^{1}=2 k_{B} T_{\perp} n / m, v_{0}^{1}=v_{1}^{1}=u_{\|}$ und $a_{0}^{1}=a_{1}^{1}=m / 2 k_{B} T_{\|}$.

In einem ersten Schritt müssen die Integrale

$$
\begin{aligned}
\int d v \frac{1}{\omega-k v \pm \Omega} \frac{\partial F}{\partial v} & =-2 \sum_{i} a_{i} F_{i} \int d v \frac{v-v_{i}}{\omega-k v \pm \Omega} e^{-a_{i}\left(v-u_{i}\right)^{2}} \\
& =2 \sum_{i} \frac{\sqrt{a_{i}} F_{i}}{k} \int d u \frac{u e^{-u^{2}}}{u-\sqrt{a_{i}}\left(\omega-k v_{i} \pm \Omega\right) / k} \\
\int d v \frac{\omega-k v}{\omega-k v \pm \Omega} F & =\sum_{i} F_{i} \int d v \frac{\omega-k v}{\omega-k v \pm \Omega} e^{-a_{i}\left(v-v_{i}\right)^{2}} \\
& =\sum_{i} F_{i} \int d v\left(1 \mp \frac{\omega}{\omega-k v \pm \Omega}\right) e^{-a_{i}\left(v-v_{i}\right)^{2}} \\
& =\sum_{i} F_{i} \sqrt{\frac{\pi}{a_{i}}} \mp \\
& \mp \sum_{i} F_{i} \frac{\Omega}{k} \int d u \frac{e^{-u^{2}}}{u-\sqrt{a_{i}}\left(\omega-k v_{i} \pm \Omega\right) / k}
\end{aligned}
$$

und

$$
\begin{aligned}
\int d v \frac{v}{\omega-k v} \frac{\partial F}{\partial v} & =\int d v \frac{1}{k}\left(\frac{\omega}{\omega-k v}-1\right) \frac{\partial F}{\partial v} \\
& =2 \sum_{i} \frac{F_{i} \sqrt{a_{i}} \omega}{k^{2}} \int d u \frac{u e^{-u^{2}}}{u-\sqrt{a_{i}}(\omega-k v) / k}
\end{aligned}
$$

berechnet werden, wobei die Indizes für die Teilchenspezies $s$, die Ordnung $n$ und die Bezugsrichtung $\|$ an dieser Stelle fallen gelassen wurden. Darin lassen sich, solange $\operatorname{Im} \zeta>0$ gilt,

$$
\int d u \frac{e^{-u^{2}}}{u-\zeta}=\sqrt{\pi} Z(\zeta)
$$

und

$$
\begin{aligned}
\int d u \frac{u e^{-u^{2}}}{u-\zeta} & =\int d u e^{-u^{2}}+\zeta \int d u \frac{e^{-u^{2}}}{u-\zeta} \\
& =\sqrt{\pi}(1+\zeta Z(\zeta)) \\
& =-\frac{\sqrt{\pi}}{2} Z^{\prime}(\zeta)
\end{aligned}
$$

auf die nicht-relativistische Plasmadispersionsfunktion zurückführen. Diese spezielle Funktion drückt sich durch die komplexe Fehlerfunktion aus und ist in der ganzen komplexen Ebene analytisch (s. Abschnitt 2.2 und die dortigen Zitate). Damit ergeben sich zusammen mit den Abkürzungen

$$
\zeta_{s, n, \pm}^{i}=\sqrt{a_{s, n}^{i}} \frac{\omega-k_{\|} v_{s, n}^{i} \pm \Omega_{s}}{k_{\|}}
$$


und

$$
\zeta_{s, n, 0}^{i}=\sqrt{a_{s, n}^{i}} \frac{\omega-k_{\|} v_{s, n}^{i}}{k_{\|}}
$$

sofort die Ergebnisse

$$
\begin{aligned}
D_{\|}\left(\omega, k_{\|}\right) & =\omega^{2}+\sum_{s} \frac{\omega_{p s}^{2}}{n_{s}} \int d v_{\|} \frac{\omega v_{\|}}{\omega-k_{\|} v_{\|}} \frac{\partial F_{0, s}}{\partial v_{\|}} \\
& =\omega^{2}-\sum_{s, i} \frac{\omega_{p s}^{2}}{n_{s}} F_{s, 0}^{i} \frac{a_{s, 0}^{i} \omega^{2}}{k_{\|}^{2}} Z^{\prime}\left(\zeta_{s, 0,0}^{i}\right)
\end{aligned}
$$

und

$$
\begin{aligned}
D_{ \pm}\left(\omega, k_{\|}\right)= & \omega^{2}-c^{2} k_{\|}^{2} \\
& +\sum_{s} \frac{\omega_{p s}^{2}}{n_{s}} \int d v_{\|} \frac{k_{\|} / 2}{\omega-k_{\|} v_{\|} \pm \Omega_{s}} \frac{\partial F_{s, 1}}{\partial v_{\|}} \\
& -\sum_{s} \frac{\omega_{p s}^{2}}{n_{s}} \int d v_{\|} \frac{\omega-k_{\|} v_{\|}}{\omega-k_{\|} v_{\|} \pm \Omega_{s}} F_{0, s} \\
= & \omega^{2}-c^{2} k_{\|}^{2} \\
& +\sum_{s, i} \frac{\omega_{p s}^{2}}{n_{s}} a_{s, 1}^{i} F_{s, 1}^{i}\left(1+\zeta_{s, 1, \pm}^{i} Z\left(\zeta_{s, 1, \pm}^{i}\right)\right) \\
& -\sum_{s, i} \frac{\omega_{p s}^{2}}{n_{s}} F_{s, 0}^{i}\left(1 \pm \frac{\sqrt{a_{s, 0}^{i}} \Omega_{s}}{k_{\|}} Z\left(\zeta_{s, 0, \pm}^{i}\right)\right)
\end{aligned}
$$

wenn $\operatorname{Im} \omega>0$ und $k_{\|}>0$ erfüllt sind. Für $k_{\|}<0$ und $\operatorname{Im} \omega>0$ muß in den Gleichungen D.8, D.11 und D.13 das mit der Plasmadispersionsfunktion verbundene Integral so umgeschrieben werden, daß wiederum $\operatorname{Im} \zeta_{s, n, \pm}^{i}>0$ und $\operatorname{Im} \zeta_{s, n, 0}^{i}>0$ gelten. Auf diese Weise ergeben sich die Symmetrien

$$
D_{\|}\left(\omega, k_{\|}\right)=D_{\|}\left(-\omega^{*},-k_{\|}\right)^{*}
$$

und

$$
D_{ \pm}\left(\omega, k_{\|}\right)=D_{\mp}\left(-\omega^{*},-k_{\|}\right)^{*} .
$$

Die Dispersionsfunktionen $D_{\|}$und $D_{ \pm}$sind für $\operatorname{Im} \omega<0$ durch analytische Fortsetzung definiert. 



\section{Literaturverzeichnis}

Abramowitz, M. und Stegun, I. A., Handbook of mathematical functions, Dover Publications, New York, 1965.

Aellig, M. R., Lazarus, A. J. und Steinberg, J. T., The solar wind helium abundance: Variation with wind speed and the solar cycle, Geophysical Research Letters, 28, S. 2767-2770, 2001.

Alfvén, H., Granulation, magneto-hydrodynamic waves, and the heating of the solar corona, Monthly Notices of the Royal Astronomical Society, 107, S. 211-219, 1947.

Altman, N. S., Kernel smoothing of data with correlated errors, Journal of the American Statistical Association, 85, S. 749-759, 1990.

Arunasalam, V., Quasilinear theory of ion-cyclotron-resonance heating of plamas and associated longitudinal cooling, Physical Review Letters, 37, S. 746-749, 1976.

Asbridge, J. R., Bame, S. J. und Feldman, W. C., Abundance differences in solar wind double streams, Solar Physics, 37, S. 451-467, 1974.

Axford, W. I. und McKenzie, J. F., The origin of high speed solar wind streams, Solar Wind Seven, (Hg.) Marsch, E. und Schwenn, R., S. 1-5, 1992.

Axford, W. I. und McKenzie, J. F., The solar wind, Cosmic winds and the heliosphere, (Hg.) Jokipii, J. R., Sonett, C. P. und Giampapa, M. S., S. 31-66, The University of Arizona Press, 1997.

Bame, S. J., Asbridge, J. R., Feldman, W. C., Gary, S. P. und Montgomery, M. D., Evidence for local ion heating in solar wind high speed streams, Geophysical Research Letters, 2, S. 373-375, 1975.

Bame, S. J., McComas, D. J., Barraclough, B. L., Phillips, J. L., Sofaly, K. J., Chavez, J. C., Goldstein, B. E. und Sakurai, R. K., The Ulysses solar wind plasma experiment, Astronomy and Astrophysics Supplement Series, 92, S. 237-265, 1992.

Belcher, J. W., Alfvénic wave pressure and the solar wind, Astrophysical Journal, 168, S. 509-524, 1971.

Belcher, J. W. und Davis, L., Large-amplitude Alfvén waves in the interplanetary medium, Journal of Geophysical Research, 76, S. 3534-3563, 1971. 
Belcher, J. W., Bridge, H. S., Lazarus, A. J. und Sullivan, J. D., Preliminary results from the Voyager solar wind experiment, Solar Wind Four, (Hg.) Rosenbauer, H., S. 131$142,1981$.

Biermann, L., Kometenschweife und solare Korpuskularstrahlung, Zeitschrift für Astrophysik, 29, S. 274-286, 1951.

Brambilla, M., Kinetic theory of plasma waves: Homogeneous plasmas, The international series of monographs on physics, Bd. 96, Oxford: Clarendon Press, 1998.

Daughton, W. und Gary, S. P., Electromagnetic proton/proton instabilities in the solar wind, Journal of Geophysical Research, 103, S. 20,613-20,620, 1998.

Daughton, W., Gary, S. P. und Winske, D., Electromagnetic proton/proton instabilities in the solar wind: Simulations, Journal of Geophysical Research, 104, S. 4657-4667, 1999.

Davidson, R. C., Methods in nonlinear plasma theory, Pure and applied physics, Bd. 37, Academic Press, 1972.

Davidson, R. C., Kinetic waves and instabilities in a uniform plasma, Basic plasma physics, Handbook of plasma physics, Bd. 1, (Hg.) Galeev, A. A. und Sudan, R. N., S. 519-585, North-Holland Publishing Company, 1983.

Davidson, R. C. und Ogden, J. M., Electromagnetic ion cyclotron instability driven by ion energy anisotropy in high-beta plasmas, Physics of Fluids, 18, S. 1045-1050, 1975.

Denskat, K. U. und Neubauer, F. M., Observations of hydromagnetic turbulence in the solar wind, Solar Wind Five, (Hg.) Neugebauer, M., S. 81-92, 1983.

Drummond, W. und Pines, D., Non-linear stability of plasma oszillations, Nucl. Fusion Suppl., 3, S. 1049-1057, 1962.

Dum, C. T., Marsch, E. und Pilipp, W., Determination of wave growth from measured distribution functions and transport theory, Journal of Plasma Physics, 23, S. 91-113, 1980.

Feldman, W. C., Asbridge, J. R., Bame, S. J. und Montgomery, M. D., Double ion stream in the solar wind, Journal of Geophysical Research, 78, S. 2017-2027, 1973.

Feldman, W. C., Asbridge, J. R., Bame, S. J. und Montgomery, M. D., Interpenetrating solar wind streams, Reviews of Geophhysics and Space Physics, 12, S. 715-723, 1974.

Feldman, W. C., Asbridge, J. R., Bame, S. J. und Gosling, J. T., Long-term variations of selected solar wind properties: IMP 6,7, and 8 results, Journal of Geophysical Research, 83, S. 2177-2189, 1978.

Fried, B. D. und Conte, S. D., The Plasma Dispersion Function, The Plasma Dispersion Function, New York: Academic Press, 1961, 1961. 
Galeev, A. A. und Sagdeev, R. Z., Wave-particle interaction, Basic plasma physics, Handbook of plasma physics, Bd. 1, (Hg.) Galeev, A. A. und Sudan, R. N., S. 683-698, North-Holland Publishing Company, 1983a.

Galeev, A. A. und Sagdeev, R. Z., Wave-wave interaction, Basic plasma physics, Handbook of plasma physics, Bd. 1, (Hg.) Galeev, A. A. und Sudan, R. N., S. 699-711, North-Holland Publishing Company, 1983b.

Galeev, A. A. und Sagdeev, R. Z., Wave-particle-wave interaction, Basic plasma physics, Handbook of plasma physics, Bd. 1, (Hg.) Galeev, A. A. und Sudan, R. N., S. 713-731, North-Holland Publishing Company, 1983c.

Gary, S. P., Theory of space plasma microinstabilities, Cambridge atmospheric and space science series, Cambridge University Press, 1993.

Gary, S. P., Madland, C. D. und Tsurutani, B. T., Electromagnetic ion beam instabilities II, Physics of Fluids, 28, S. 3691-3695, 1985.

Gary, S. P., Madland, C. D., Schriver, D. und Winske, D., Computer simulations of electromagnetic cool ion beam instabilities, Journal of Geophysical Research, 91, S. 41884200, 1986.

Gasser, T. und Müller, H. G., Kernel estimation of regression functions, Smoothing techniques for curve estimation, Lecture Notes in Mathematics, Bd. 757, S. 23-68, SpringerVerlag, Berlin, 1979.

Goldstein, B. E., Neugebauer, M., Zhang, L. D. und Gary, S. P., Observed constraints on proton-proton velocities in the solar wind, Geophysical Research Letters, 27, S. 53-56, 2000 .

Goodrich, C. C., Wave-particle interactions and the dynamics of the solar wind, Dissertation, Massachusetts Institute of Technology, 1978.

Hand, D. J., Kernel discriminant analysis, Pattern recognition and image processing research studies series, Research Studies Press, 1982.

Härdle, W. und Gasser, T., Robust nonparametric function fitting, Journal of the Royal Statistical Society, B 46, S. 42-51, 1984.

Hartle, R. E. und Sturrock, P. A., Two-fluid model of the solar wind, Astrophysical Journal, 151, S. 1155-1170, 1968.

Hasegawa, A., Drift mirror instability in the magnetosphere, Physics of Fluids, 12, S. 2642-2650, 1969.

Hollweg, J. V., Alfvén waves in a two-fluid model of the solar wind, Astrophysical Journal, 181, S. 547-566, 1973.

Hollweg, J. V., On electron heat conduction in the solar wind, Journal of Geophysical Research, 79, S. 3845-3850, 1974. 
Hollweg, J. V., Collisionless electron heat conduction in the solar wind, Journal of Geophysical Research, 81, S. 1649-1658, 1976.

Hollweg, J. V., Some physical processes in the solar wind, Reviews of Geophysics and Space Physics, 16, S. 689-720, 1978.

Hu, Y. Q. und Habbal, S. R., Resonant acceleration and heating of solar wind ions by dispersive ion cyclotron waves, Journal of Geophysical Research, 104, S. 17,045-17,056, 1999.

Hu, Y. Q., Esser, R. und Habbal, S. R., A fast solar wind model with anisotropic proton temperature, Journal of Geophysical Research, 102, S. 14,661-14,676, 1997.

Hu, Y. Q., Habbal, S. R. und Li, X., On the cascade process of Alfvén waves in the fast solar wind, Journal of Geophysical Research, 104, S. 24,819-24,834, 1999.

Hu, Y. Q., Esser, R. und Habbal, S. R., A four-fluid turbulence driven solar wind model for preferential acceleration and heating of heavy ions, Journal of Geophysical Research, 105, S. 5093-5111, 2000.

Isenberg, P. A., Resonant acceleration and heating of solar wind ions: Anisotropy and dispersion, Journal of Geophysical Research, 89, S. 6613-6622, 1984.

Isenberg, P. A., Heating of coronal holes and generation of the solar wind by ion-cyclotron resonance, Space Science Reviews, 95, S. 119-131, 2001a.

Isenberg, P. A., The kinetic shell model of coronal heating and acceleration by ion cyclotron waves. 1. Outward propagating waves, Journal of Geophysical Research, 106, S. 5649-5660, 2001b.

Isenberg, P. A., The kinetic shell model of coronal heating and acceleration by ion cyclotron waves. 2. Inward and outward propagating waves, Journal of Geophysical Research, 106, S. 29,249-29,260, 2001c.

Isenberg, P. A. und Hollweg, J. V., Finite amplitude Alfvén waves in a multi-ion plasma: Propagation, acceleration, and heating, Journal of Geophysical Research, 87, S. 50235029, 1982.

Isenberg, P. A. und Hollweg, J. V., On the preferential acceleration and heating of solar wind heavy ions, Journal of Geophysical Research, 88, S. 3923-3935, 1983.

Isensee, U., Plasma disturbances caused by the Helios spacecraft in the solar wind, Journal of Geophysics, 42, S. 581-589, 1977.

Isensee, U. und Maaßberg, H., Plasma in cell simulation of the plasma environment of a spacecraft in the solar wind, Advances in Space Research, 1, S. 413-416, 1981.

Jacques, S. A., Momentum and energy transport by waves in the solar atmosphere and solar wind, Astrophysical Journal, 215, S. 942-951, 1977. 
Jacques, S. A., Solar wind models with Alfvén waves, Astrophysical Journal, 226, S. 632-649, 1978.

Kennel, C. F. und Engelmann, F., Velocity space diffusion from weak plasma turbulence in a magnetic field, Physics of Fluids, 9, S. 2377-2388, 1966.

Leer, E. und Axford, W. I., A two fluid solar wind model with anisotropic proton temperature, Solar Physics, 23, S. 238-250, 1972.

Leer, E. und Holzer, T. E., Energy addition in the solar wind, Journal of Geophysical Research, 85, S. 4681-4688, 1980.

Leubner, M. P. und Viñas, A. F., Stability analysis of double-peaked proton distribution functions in the solar wind, Journal of Geophysical Research, 91, S. 13,366-13,372, 1986.

Li, X., Habbal, S., Esser, R. und Hu, Y. Q., Influence of heavy ions on the high-speed solar wind, Journal of Geophysical Research, 102, S. 17,419-17,432, 1997.

Li, X., Habbal, S., Hollweg, S. V. und Esser, R., Heating and cooling of protons by turbulence-driven ion cyclotron waves in the fast solar wind, Journal of Geophysical Research, 104, S. 2521-2535, 1999.

Livi, S. und Marsch, E., Generation of solar wind proton tails and double beams by Coulomb collision, Journal of Geophysical Research, 92, S. 7255-7261, 1987.

Marsch, E. und Livi, S., Observational evidence for marginal stability of solar wind ion beams, Journal of Geophysical Research, 92, S. 7263-7268, 1987.

Marsch, E. und Tu, C.-Y., Evidence for pitch angle diffusion of solar wind protons in resonance with cyclotron waves, Journal of Geophysical Research, 106, S. 8357-8361, 2001.

Marsch, E., Pilipp, W., Rosenbauer, H., Schwenn, R. und Mühlhauser, K.-H., Some characteristics of proton velocity distributions in the solar wind as observed by the Helios solar probes, Solar Wind Four, (Hg.) Rosenbauer, H., S. 273-279, 1981.

Marsch, E., Goertz, C. K. und Richter, K., Wave heating and acceleration of solar wind ions by cyclotron resonance, Journal of Geophysical Research, 87, S. 5030-5044, 1982a.

Marsch, E., Mühlhäuser, K.-H., Rosenbauer, H., Schwenn, R. und Neubauer, F. M., Solar wind helium ions: Observations of the Helios solar probes between 0.3 and $1 \mathrm{AU}$, Journal of Geophysical Research, 87, S. 35-51, 1982b.

Marsch, E., Mühlhauser, K.-H., Schwenn, R., Rosenbauer, H., Pilipp, W. und Neubauer, F. M., Solar wind protons: Three-dimensional velocity distributions and derived plasma parameters measured between 0.3 and 1 AU, Journal of Geophysical Research, 87, S. $52-72,1982 \mathrm{c}$. 
Marsch, E., Mühlhäuser, K. H., Rosenbauer, H. und Schwenn, R., On the equation of state of solar wind ions derived from HELIOS measurements, Journal of Geophysical Research, 88, S. 2982-2992, 1983.

Marsch, E., Ao, X.-Z. und Tu, C.-Y., On the temperature anisotropy of the core part of the proton velocity distribution function in the solar wind, Journal of Geophysical Research, 109, S. 4102-4109, 2004.

McKenzie, J. F., Ip, W.-H. und Axford, W. I., The acceleration of minor ion species in the solar wind, Astrophysics and Space Science, 64, S. 183-211, 1979.

Montgomery, M. D., Gary, S. P., Forslund, D. W. und Feldman, W. C., Electromagnetic ion-beam instabilities in the solar wind, Physical Review Letters, 35, S. 667-670, 1975.

Montgomery, M. D., Gary, S. P., Feldman, W. C. und Forslund, D. W., Electromagnetic instabilities driven by unequal proton beams in the solar wind, Journal of Geophysical Research, 81, S. 2743-2749, 1976.

Musmann, G., Neugebauer, F. M., Maier, A. und Lammers, E., Das FörstersondenMagnetfeldexperiment (E2), Raumfahrtforschung, 15, S. 232-237, 1975.

Neugebauer, M. und Snyder, C. W., The mission of Mariner II: Preliminary observations, Science, 138, S. 1095-1097, 1962.

Ogilvie, K. W. und Hirshberg, J., The solar cycle variation of the solar wind helium abundance, Journal of Geophysical Research, 79, S. 4595-4602, 1974.

Parker, E. N., Dynamics of the interplanetary gas and magnetic fields, Astrophysical Journal, 128, S. 644-676, 1958.

Parker, E. N., The hydrodynamic theory of solar corpuscular radiation and stellar winds, Astrophysical Journal, 132, S. 821-866, 1960.

Parker, E. N., Dynamical properties of stellar coronas and stellar winds. I. Integration of the momentum equation, Astrophysical Journal, 139, S. 72-92, 1963a.

Parker, E. N., Dynamical properties of stellar coronas and stellar winds. II. Integration of the heat flow equation, Astrophysical Journal, 139, S. 93-123, $1963 \mathrm{~b}$.

Parker, E. N., Dynamical properties of stellar coronas and stellar winds. III. Dynamics of coronal streamers, Astrophysical Journal, 139, S. 690-709, 1963c.

Parker, E. N., Dynamical properties of stellar coronas and stellar winds. IV. The separate existence of subsonic and supersonic solutions, Astrophysical Journal, 141, S. 1463$1478,1965$.

Pilipp, W., Schwenn, R., Marsch, E. und Mühlhauser, K.-H., Electron characteristics in the solar wind as deduced from Helios observations, Solar Wind Four, (Hg.) Rosenbauer, H., S. 241-249, 1981. 
Pilipp, W. G., Miggenrieder, H., Mühlhäuser, K.-H., Rosenbauer, H. und Schwenn, R., Data analysis of electron measurements of the plasma experiment aboard the Helios probes, Techn. Ber. 185, Max-Plack-Institut für Physik und Astrophysik, MPE Report, 1984.

Pilipp, W. G., Miggenrieder, H., Montgomery, M. D., K.-H. Mühlhäuser, H. R. und Schwenn, R., Characteristics of electron velocity distribution functions in the solar wind derived from the Helios plasma experiment, Journal of Geophysical Research, 92, S. 1075-1092, 1987a.

Pilipp, W. G., Miggenrieder, H., Mühlhäuser, K.-H., Rosenbauer, H., Schwenn, R. und Neubauer, F. M., Variations of electron distribution functions in the solar wind, Journal of Geophysical Research, 92, S. 1103-1118, $1987 \mathrm{~b}$.

Porsche, H., General Aspects of the Mission Helios 1 and 2, Journal of Geophysics, 42, S. 551-559, 1977.

Rosenbauer, H., Possible effects of photoelectron emission on a low energy electron experiment, ASSL Photon and particle interaction with surfaces in space, Astrophysics and space science library, Bd. 37, (Hg.) Grard, R. J. L., S. 139-151, 1973.

Rosenbauer, H., Schwenn, R., Marsch, E., Meyer, B., Miggenrieder, H., Montgomery, M. D., Mühlhäuser, K. H., Pilipp, W., Voges, W. und Zink, S. M., General Aspects of the Mission Helios 1 and 2, Journal of Geophysics, 42, S. 561-580, 1977.

Rosenbauer, H., Schwenn, R., Miggenrieder, H., Meyer, B., Grünwaldt, H., Mühlhäuser, K.-H., Pellkofer, H. und Wolfe, J. H., Die Instrumente des Plasmaexperiments auf den HELIOS-Sonnensonden, Techn. Ber. W 81-015, Bundesministerium für Forschung und Technik, 1981.

Sagdeev, R. Z. und Galeev, A. A., Nonlinear Plasma Theory, Benjamin, 1969.

Schwartz, S. J. und Marsch, E., The radial evolution of a single solar wind plasma parcel, Journal of Geophysical Research, 88, S. 9919-9932, 1983.

Schwartz, S. J., Feldman, W. C. und Gary, S. P., The source of proton anisotropy in the high-speed solar wind, Journal of Geophysical Research, 86, S. 541-546, 1981.

Scott, D. W., Multivariate density estimation. Theory, practice and visualization, Wiley series in probability and mathematical statistics, Wiley, 1992.

Scott, D. W., Tapia, R. A. und Thompson, J. R., Kernel density estimation revisited, Journal of nonlinear analysis, theory, methods, and application, 1, S. 339-372, 1977.

Silverman, B. W., Density estimation for statistics and data analysis, Monographs on statistics and applied probability, Chapman and Hall, 1986.

Smith, C. W. und Bieber, J. W., Solar cycle variation of the interplanetary magnetic field spiral, Astrophysical Journal, 370, S. 435-441, 1991. 
Southwood, D. J. und Kivelson, M. G., Mirror instability. I - Physical mechanism of linear instability, Journal of Geophysical Research, 98, S. 9181-9187, 1993.

Stix, T. H., Waves in plasmas, Springer, AIP Press, 1992.

Sturrock, P. A. und Hartle, R. E., Two-fluid model of the solar wind, Physical Review Letters, 16, S. 628-631, 1966.

Tsurutani, B. T., Southwood, D. J., Smith, E. J. und Balogh, A., Nonlinear magnetosonic waves and mirror mode structures in the march 1991 Ulysses interplanetary event, Geophysical Research Letters, 19, S. 1267-1270, 1992.

Tu, C.-Y., A solar wind model with the power spectrum of Alfvénic fluctuations, Solar Physics, 109, S. 149-186, 1987.

Tu, C.-Y. und Marsch, E., Comment on "Evolution of energy-containing turbulent eddies in the solar wind"by W.H. Matthaeus, S. Oughton, D.H. Pontius Jr. and Y. Zhou, J. Geophys. Res., 100, S. 12,323-12,328, 1995.

Tu, C.-Y. und Marsch, E., Anisotropy regulation and plateau formation through pitch angle diffusion of solar wind protons in resonance with ion-cyclotron waves, Journal of Geophysical Research, 107, S. SSH 7, 2002.

Tu, C.-Y., Pu, Z.-Y. und Wei, F.-S., The power spectrum of interplanetary Alfvénic fluctuations: Derivation of the governing equation and its solution, Journal of Geophysical Research, 89, S. 9695-9702, 1984.

Tu, C.-Y., Wang, L.-H. und Marsch, E., Formation of the proton beam distribution in high-speed solar wind, Journal of Geophysical Research, 107, S. SSH 8, 2002.

Tu, C.-Y., Marsch, E. und Qin, Z.-R., Dependence of the proton beam drift velocity on the proton core plasma beta in the solar wind, Journal of Geophysical Research, 109, S. A05,101, 2004.

Vedenov, A., Velikov, E. und Sagdeev, R., not available, Nucl. Fusion Suppl., 2, S. 465$475,1962$.

Voigt, G.-H., Isensee, U. und Maaßberg, H., Die Wechselwirkungen der Helios-Sonde mit dem Plasma des solaren Windes, Techn. Ber. W 81-036, Bundesministerium für Forschung und Technik, 1981.

von Steiger, R., Geiss, J. und Gloeckler, G., Composition of the solar wind, Cosmic winds and the heliosphere, (Hg.) Jokipii, J. R., Sonett, C. P. und Giampapa, M. S., S. 581-616, The University of Arizona Press, 1997.

Watson, L. T., Billups, S. C. und Morgan, A. P., Algorithm 652. Hompack: A suite of codes for globally convergent homotopy algorithms, ACM Transactions on Mathematical Software, 13, S. 281-310, 1987.

Wenzel, K.-P., Marsden, R. G., Page, D. E. und Smith, E. J., Ulysses: The first highlatitude heliospheric mission, Advances in Space Research, 9, S. 25-29, 1989. 
Zurbuchen, T. H., Hefti, S., Fisk, L. A., Gloeckler, G., Schwadron, N. A., Smith, C. W., Ness, N. F., Skoug, R. M., McComas, D. J. und Burlaga, L. F., On the origin of microscale magnetic holes in the solar wind, Journal of Geophysical Research, 106, S. 16,001-16,010, 2001. 



\section{Danksagung}

Die vorliegende Arbeit wurde am Max-Planck-Institut für Sonnensystemforschung in Katlenburg-Lindau bei Göttingen angefertigt. Ich bedanke mich bei der Max-PlanckGesellschaft für die Bereitstellung eines Arbeitsplatzes an diesem Institut und für die Bewilligung eines Stipendiums.

Ich möchte mich bei Prof. E. Marsch für die Betreuung dieser Arbeit und seine große Bereitschaft zu wissenschaftlichen Diskussionen bedanken, für die er jederzeit ansprechbar gewesen ist.

Mein Dank gilt desweiteren Prof. F. Kneer für die Betreuung der Arbeit seitens der Universität Göttingen. Ich bedanke mich bei ihm insbesondere für sein Engagement als Referent dieser Arbeit. 



\section{Lebenslauf}

\section{Persönliche Daten}

Name:

Geburtstag:

Geburtsort:

Staatsangehörigkeit:

\section{Schulbildung}

1982 - 1986

$1986-1988$

$1988-1995$

\section{Studium}

Oktober 1995 - November 2000

04.07.1997

16.10.1997

10.11.2000

Titel der Diplomarbeit:

April 2001 - März 2004

\author{
Michael Heuer \\ 16.11.1975 \\ Peine \\ Deutsch
}

Grundschule in Peine

Orientierungsstufe in Peine

Gymnasium Groß Ilsede, Abitur im Mai 1995

Studium der Physik und Mathematik an der Universität Göttingen

Vordiplom Mathematik

Vordiplom Physik

Abschluß des Physikstudiums mit dem Diplom im Fachgebiet Theoretische Physik

AdS/CFT-Korrespondenz in zwei Dimensionen

Stipendiat am Max-Planck-Institut für Sonnensystemforschung 